\title{
Improving procedural sedation and/or analgesia in children : from practice over evidence to practice
}

Citation for published version (APA):

Leroy, P. L. J. M. (2012). Improving procedural sedation and/or analgesia in children : from practice over evidence to practice. [Doctoral Thesis, Maastricht University]. Datawyse / Universitaire Pers Maastricht. https://doi.org/10.26481/dis.20120302pl

Document status and date:

Published: 01/01/2012

DOI:

10.26481/dis.20120302pl

Document Version:

Publisher's PDF, also known as Version of record

\section{Please check the document version of this publication:}

- A submitted manuscript is the version of the article upon submission and before peer-review. There can be important differences between the submitted version and the official published version of record.

People interested in the research are advised to contact the author for the final version of the publication, or visit the DOI to the publisher's website.

- The final author version and the galley proof are versions of the publication after peer review.

- The final published version features the final layout of the paper including the volume, issue and page numbers.

Link to publication

\footnotetext{
General rights rights.

- You may freely distribute the URL identifying the publication in the public portal. please follow below link for the End User Agreement:

www.umlib.nl/taverne-license

Take down policy

If you believe that this document breaches copyright please contact us at:

repository@maastrichtuniversity.nl

providing details and we will investigate your claim.
}

Copyright and moral rights for the publications made accessible in the public portal are retained by the authors and/or other copyright owners and it is a condition of accessing publications that users recognise and abide by the legal requirements associated with these

- Users may download and print one copy of any publication from the public portal for the purpose of private study or research.

- You may not further distribute the material or use it for any profit-making activity or commercial gain

If the publication is distributed under the terms of Article $25 \mathrm{fa}$ of the Dutch Copyright Act, indicated by the "Taverne" license above, 


\section{Improving Procedural Sedation and/or Analgesia in Children}

- from practice over evidence to practice - 
Printing of this thesis was partiallly supported by:

- Stichting Spoedeisende Hulp bij Kinderen (www.sshk.nl)

- Linde Health Care Benelux

- The Surgical Company B.V.

-Vygon Nederland B.V.

Cover Design by Bilbo Schickenberg (www.bilbos.nl)

The painting on the wall in the waiting room is "Local Anaesthesia" (1989) by Kees de Hond. This piece of art takes part of an artistic statement on the managing and acting of doctors and nurses, seen through the eyes of an unprepared child, as experienced by the painter himself during his childhood. By means of special effects with the perspective, the artist creates in his drawings and paintings, the oppressing atmosphere of the events of that horrifying period. "I had to express the hidden images emerging from the subconscious, those of the innocent child - unprepared on what is going to happen - against the background of fear, sorrow, and feelings of desertion". (www.keesdehond.nl)

(C) Piet Leo Jules Maria LEROY, Maastricht 2012

ISBN 978-94-6159-120-3

Production: Datawyse | Universitaire Pers Maastricht 


\title{
Improving Procedural Sedation and/or Analgesia in Children
}

\section{- from practice over evidence to practice -}

\author{
DISSERTATION \\ to obtain the degree of Doctor at Maastricht University, \\ on the authority of the Rector Magnificus, Prof. Dr. G.P.M.F. Mols \\ in accordance with de decision of the Board of Deans, \\ to be defended in public on Friday the $2^{\text {nd }}$ of March 2012 at 14.00 hours
}

by

Piet Leo Jules Maria LEROY

Born on the $1^{\text {st }}$ of January 1969 (Deinze - Belgium)

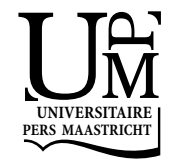




\section{Supervisors}

Prof. dr. L.J.I. Zimmermann

Prof. dr. J.T.A. Knape, Universitair Medisch Centrum Utrecht

\section{Assessment Committee}

Prof. dr. M. van Kleef (Chairman)

Prof. dr. R. de Groot, Universitair Medisch Centrum St.-Radboud Nijmegen

Prof. dr. C. Neef

Dr. H.M.M. Willigers 
Het woord

is een vrucht

waarvan de schil kletspraat heet, het vlees welsprekendheid, en de pit gezond verstand

(Maryse Condé, Ségou I)

Heureux qui chante pour l'enfant Et qui sans jamais rien lui dire le guide au chemin triomphant.

(Jaques Brel)

leder gedicht komt uit het duister voort en wordt uit twijfel en gemis geboren. Maar ook al zie je door een spiegel: ooit komt een aneonenlang verborgen woord volmaakt en stralend uit de nacht naar voren, en wordt het vers dat alles zegt voltooid

(Het Vers, JP Rawie)

Voor Rik, de eeuwige afwezige 


\title{
List of Abbreviations
}

\author{
AAP American Academy of Pediatrics \\ APLS Advanced Pediatric Life Support \\ ASA American Society of Anesthesiologists \\ BIS Bispectral Index System \\ BLS Basic Life Support \\ BP Blood Pressure \\ BW Burgerlijk Wetboek [Dutch Civil Code] \\ CBO Centraal Begeleidings Orgaan /Kwaliteitsinstituut voor de Gezondheidszorg [Dutch \\ Institute for Heathcare Improvement] \\ CT Computed Tomography \\ EACH European Association for Children in Hospital \\ EBGD Evidence-based Guideline Development \\ ECG Electrocardiography \\ ED Emergency Department \\ ETCO2 End Tidal carbo-di-oxide concentration \\ GIE Gastro-Intestinal Endoscopy \\ IGZ Inspectie voor de Gezondheidszorg [Dutch Health Care Inspectorate] \\ MMWT Modified Maintenance of Wakefulness test \\ MPC Meperidine Promethazine Chlorpromazine (lytic) cocktail \\ MRI Magnetic Resonance Imaging \\ NICE National Institute for Health and Clinical Excellence \\ NVA Nederlandse Vereniging voor Anesthesiologie [Dutch Society for Anesthesiology] \\ NVK Nederlandse Vereniging voor Kindergeneeskunde [Dutch Society for Pediatrics] \\ PCA Principle Component Analysis \\ pCO2 Partial carbo-di-oxide pressure \\ PhD Doctor of Philosophy \\ PICU Pediatric Intensive Care Unit \\ PS Procedural Sedation \\ PSA Procedural Sedation and/or Analgesia \\ PPSA Pediatric Procedural Sedation and/or Analgesia \\ RCT Randomized Controlled Trial \\ SIGN Scottish Intercollegiate Guideline Network \\ SPSS Statistical Package for the Social Sciences \\ SR Systematic Review \\ UMSS University of Michigan Sedation Scale \\ WGBO 'Wet op de Geneeskundige Behandel Overeenkomst'[Medical Treatment Contracts Act] \\ ZBC Zelfstandig Behandel Centrum [Independent Treament Centre]
}




\section{Table of Content}

CHAPTER 1 Introduction

$\begin{array}{lll}\text { Part } 1.1 & \text { The report of this thesis' journey } & 11\end{array}$

$\begin{array}{lll}\text { Part } 1.2 & \text { Historical perspectives, overview and definitions } & 23\end{array}$

CHAPTER 2 PSA in Dutch general paediatrics. An analysis of safety and effectiveness 43

Part 2.1 Adherence to safety guidelines on paediatric procedural sedation and analgesia. $\quad 45$

Part 2.2 Safe and effective procedural sedation for magnetic resonance imaging in 53 children.

Part 2.3 Safe and effective procedural sedation/analgesia (PSA) for gastro-intestinal $\quad 77$ endoscopy in children.

Part 2.4 Providing procedural sedation/analgesia in children. How satisfied are Dutch 105 general paediatricians with their personal performance?

CHAPTER 3 Professional skills and competence for safe and effective procedural sedation/analgesia in children. Recommendations based on a systematic review of the literature.

CHAPTER 4 Forced immobilization ('restraint') during medical procedures in young children. 145 An ethical and legal investigation of a common practice

CHAPTER 5 Guideline on procedural sedation and/or analgesia (psa) in children at locations outside the operating theatre. Summary of the new Dutch guideline

CHAPTER 6 Conclusions, general discussion and future perspectives.

Mapping out the path ahead

Appendix Paediatric sedation: The European experience and approach 215

$\begin{array}{lr}\text { Samenvatting } & 235\end{array}$

$\begin{array}{ll}\text { Epiloog en Dankwoord } & 247\end{array}$

$\begin{array}{ll}\text { Curriculum Vitae } & 251\end{array}$

$\begin{array}{ll}\text { List of Publications } & 253\end{array}$ 

CHAPTER 1

Introduction 



\section{Part 1.1 The report of this thesis' journey}

Most books today seem to have been written overnight from books read that day

Nicolas Chamfort (1741-1794)

\section{To search, not to research}

This PhD-thesis did not originate primarily from a specific scientific question or hypothesis. Neither did it result from the 'science-generating-process' of an existing research group fulfilling its academic duties or ambitions. Even the highly esteemed PhD degree was - at least initially - not a personal target. Becoming a PhD is generally considered the condition sine qua non for any self-respecting academic to unlock the labyrinth hiding future career prosperities and opportunities. Indubitably, the quest for the PhD grail is a tough, instructive and challenging journey, demanding patience, hard work, discipline, modesty and a continuous process of balancing between critical acclaim and denial. Clinicians, however, should take the old wisdom 'Let the cobble stay to his last' into account. Performing high-quality science as a full-time clinician bares the real risk of threatening or even damaging complex and demanding clinical responsibilities; not to mention the continuous risk of collateral damage to private and social life and personal health.

On the other hand, clinical experiences and daily practical challenges or problems may function as very strong triggers for scientific questions and research. Ideally, this research yields specific answers that can be translated subsequently to practical solutions for the original problem. The research presented in this thesis is in fact a spin-off product of such a practical search for an implementable solution of a serious and complex problem in daily paediatric practice: how to deal safely and effectively with procedure related stress, pain or non-cooperative behaviour in sick children. In modern paediatrics invasive diagnostic and therapeutic procedures are part of daily practice. Many of these procedures are painful and stressful or are impossible to perform without immobility. Despite local anaesthesia, reassurance and distraction techniques many (young) children are unable to undergo these procedures without being physically restrained. Therefore some form of sedation and/or analgesia is often indicated. In many settings procedural sedation and analgesia (PSA) has become the standard of care for the management of acute procedural pain and anxiety. Non-anaesthesiology professionals like paediatricians, neurologists, surgeons, emergency physicians, endoscopists, radiologists and dentists have been performing PSA in children for many decades. However, their PSA practice has been associated with serious problems regarding its safety and effectiveness. These problems make up the central theme of this thesis, which journey started about two decades ago. 


\section{The 'pre-history': back to the future}

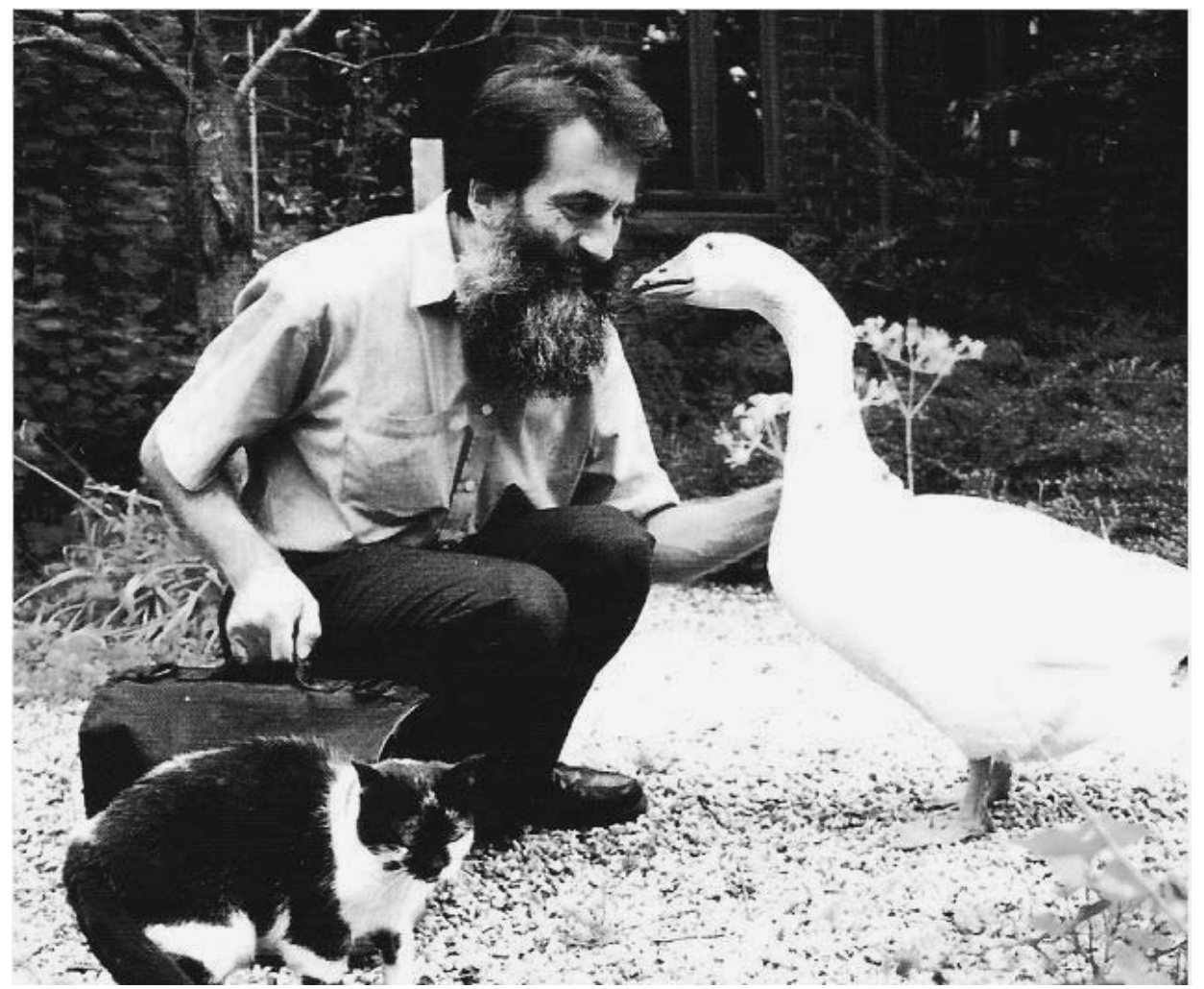

Figure 1: Dr Nikolaus Lutz-Dettinger (1956-2005)

It was the late Dr. Nikolaus Lutz-Dettinger (Figure 1), former paediatric intensivist at Ghent University Hospital (1993-1998), who during my paediatric residency drew my attention to the enormous potential impact of effective PSA on sick children's comfort and well being.

Dr Lutz-Dettinger regarded the conditions of painful procedures in paediatrics at that time as nothing else than "softly tolerated torture" (Sic). Up to the late nineties of the last century procedural comfort and reduction of procedure-related pain and anxiety were hardly regarded as relevant issues in paediatric medical care. The use of forced immobilization and restraint was (and still is) a very common and widely accepted practice during painful procedures in children. Particularly in children suffering from chronic diseases and requiring repeatedly invasive procedures (e.g. paediatric haemato-oncology patients), extreme peri-procedural stress occurred frequently. It has been shown that, for example, paediatric oncology patients regard the pain due to treatment and procedures as a greater problem than pain due to the malignant disease itself, while two thirds of the pain they experience has an iatrogenic origin. ${ }^{1}$ However, PSA was (and still is) usually restricted to procedures that threaten to fail due to patient immobility or lack of cooperation. At the paediatric oncology department, and later on also at the burn care unit and radiology department, Dr Lutz-Dettinger introduced his so-called 'optimized Ketalar ${ }^{\circledR}$ narcosis', a visionary protocol for deep, dissociatieve PSA with intravenous racemic ketamine. The impact on the quality of patient care and procedural success was impressive, as illustrated 
by the fact that paediatric nurses no longer needed to have their patients in a judoka-like hold and that anxiety-free patients entered easily the procedure room, confident that they were not about to be 'tortured' again.

As a resident I was offered the opportunity to perform many hundreds of PSA's on my own. I experienced trial-and-error-wise - in fact a common approach during my residency - that PSA was much more complex than just 'knocking out a patient' with sedatives. PSA also had to include strict safety precautions since respiratory depression or airway obstruction could occur suddenly and unpredictably. Besides, careful preparation of child and parents, quiet and childfriendly circumstances, kindly caring professionals talking with hypnosis-like tempered voices, the use of distraction techniques, adequate topical anaesthesia and individually tailored dosing of sedatives all turned out to be extremely important.

Looking back now I keep wondering why - for god's sake - we dared to perform deep sedation, which can be regarded as 'light anaesthesia', ' without having anaesthesiology expertise involved. Later on, I would find out that PSA for a very long time had been a delicate matter of debate and dispute between anaesthesiologists and non-anaesthesiologists . .

\section{PSA: non-anaesthesiologists not allowed}

In 1998 I moved to The Netherlands for my training in paediatric intensive care. As many Belgians before me I was, at first sight, easily impressed by the apparently outstanding organizational structure of the Dutch medical system. The hunt for evidence-based mutual agreement and the presence of widely supported guidelines clearly showed that this system was continuously seeking for the patient's best interests and the highest possible quality of care. The availability of a recent, authority-based Dutch guideline on PSA that was adopted by the Dutch Society of Paediatrics as their official guideline on this topic, was an obvious confirmation of that joy (CBO 1998). ${ }^{3}$

However, a proof for real implementation of the guideline was hard to find in daily practice: despite the widespread presence of the guideline booklet on the doctor's office's bookshelves, a functional paediatric PSA service was not traceable in any of the hospitals I worked. Impressively, the 80 pages counting CBO guideline included only half a page (i.e. 16 lines) on PSA in children. I was about to discover that PSA in children had an aura of taboo and disagreement. The following anecdote nicely illustrates the general opinion at that time.

One night, being on call, I was asked for advice in a 14-year-old boy acutely admitted at the oncology ward for acute leukaemia. Because of extreme hyperleucocytosis he needed plasmapheresis urgently, making a central venous access necessary. He was scheduled for anaesthesia but the intervention was postponed repeatedly because of insufficient operation room facilities. Following difficult venous access and a bone marrow puncture performed earlier that day without sedation and/or analgesia he was now too anxious to undergo any additional painful procedure. Topical anaesthesia alone was not likely to be successful. At that time I decided to perform a PSA with ketamine in the procedure room at the oncology ward. The patient was monitored continuously by cardio-respiratory monitoring and pulsoximetry. Once deeply sedated a subclavian central venous line was inserted quickly and successfully and within one hour the boy was fully awake and the requested treatment could be started. 
The next day however I was called to account by the head of anaesthesiology: the anaesthetic ketamine was restricted to use by anaesthesiologists only and therefore my action was blameworthy. My arguments referring to my former experience, the safety precautions I had applied, the need for an urgent and comfortable solution in this patient and the absence of any practical alternative were all waved aside. PSA was just considered to be beyond my competence.

Gradually I realized that, despite the availability of an excellent guideline, PSA (in children) was a serious but somewhat hidden, neglected and even negated problem in Dutch (paediatric) medicine, leading to procedural failure, patient discomfort and patient unsafety.

\section{The Paediatric PSA Unit: an innovative initiative}

In 2002, the Paediatric Intensive Care Unit (PICU) of the Maastricht University Medical Centre was the first to set up a unit for Paediatric Procedural Sedation and Analgesia (PPSA-unit), managed by non-anaesthesiologists, in The Netherlands. The immediate cause of this initiative was an anxious child suffering from acute pancreatitis and in need for daily peritoneal centesis because of relapsing ascites. During his stay on the PICU punctures were performed under deep sedation with ketamine, supervised by a paediatric intensivist. However, after he was discharged to the general ward, punctures were tried in vain under midazolam sedation. Therefore we decided to readmit him to the PICU for deep sedation each time he needed a new centesis. Obviously, very soon the question arose why this technique could not be extended to all hospitalized kids undergoing similar painful procedures and in need for a proper comfort measure. Happily for us - and only a few months after being reprimanded for performing 'illegal and unauthorized PSA' - we found some visionary anaesthesiologists who were willing to explore the boundaries of their profession. In collaboration with Professor Marcel Durieux, head of the anaesthesiology department at that time, and paediatric anaesthesiologists Brian Anderson and Henriette Willigers, a protocol was composed that was fundamentally based on national and international safety guidelines on PSA. ${ }^{4-6}$ This protocol was remarkable, mainly because the protocol text defined that in selected patient categories children can be sedated safely, including deep sedation, by trained non-anaesthesiologists as long as optimal safety preconditions and specific professional competencies were fulfilled. The fact that non-anaesthesiologists were formally 'credentialed' for PSA by anaesthesiologists was highly innovative and turned out to be the cornerstone of this project's success. After approval by the board of hospital directors, the first patient entered our PSA unit in September 2002. Since then, we performed over 700 PSA sessions successfully and safely. Initially, only haemato-oncology patients were admitted but quite soon the target population was expanded to $\mathrm{ASA}^{1}$ I or II patients fulfilling inclusion criteria and in need for deep sedation, e.g. (burn) wound treatment, diagnostic lumbar puncture and muscular or liver biopsy. This initiative received broad attention by the national press (e.g. De Volkskrant (14th of November 2008) and Hart van Nederland ${ }^{2}\left(18^{\text {th }}\right.$ of November 2008)) and was

\footnotetext{
${ }^{1}$ Physical status Classification by the American Society of Anesthesiologists (ASA)

${ }^{2}$ http://www.hartvannederland.nl/nederland/limburg/2008/slaapdokter-brengt-kinderen-in-dromenland.
} 
awarded a first price for oral presentation on the $4^{\text {th }}$ Paediatric Sedation Course (London, June 2007).

\section{Discovering the real world: the fatally failing PSA paradox}

The official status and transparency, the intense collaboration with the anaesthesiology department and the scientific way of data registration all made it possible to give publicity to the new PPSA-unit. In November 2005 we presented the results of the first 114 PSA sessions at the annual conference of the Dutch Society for Paediatrics (NVK). Strikingly the result of this presentation was like scratching of a thin scab over an overripe abscess: during the discussions following the presentation it became clear that PSA in Dutch paediatrics was associated with major problems. Paediatricians reported procedural failure resulting from ineffective PSA practices and/or a lack of anaesthetic service as a highly relevant problem in their daily practice. This was especially the case in children undergoing Magnetic Resonance Imaging. Furthermore it turned out that, since the publication of the CBO guideline in 1998, at least 3 severe accidents ( 2 with a fatal outcome and 1 with permanent damage) had happened in children during PSA. Health Inspectorate's investigation clearly showed that existing safety guidelines were not implemented in these cases. Many informal contacts with paediatricians and paediatric residents confirmed to me that these events were in no way isolated incidents but rather an indication for widespread non-adherence to established safety guidelines. These contacts generated an overall impression of a non-transparent PSA practice managed by non-competent professionals applying lowly effective but potentially dangerous sedatives. Particularly residents reported to me all kinds of near-incidents and sometimes shocking PSA practices they witnessed or had to perform without having had any formal training (Table 1). Currently, PSA is still not formally incorporated in the training of Dutch paediatricians and a particular final attainment level is non-existing.

Putting it somewhat over-simplified, this situation can be best summarized as a sinister paradox: untrained and incompetent professionals performing PSA out of sheer necessity or obstinacy versus trained and competent professionals (i.e. anaesthesiologists) not able or not willing to offer an appropriate PSA service; a dramatic condition resulting in non-transparency, ineffectiveness and, most outrageous at all, patient unsafety. This PSA paradox, explainable from a historical point of view (see part 2 of this chapter), can be hold directly responsible for the fatal accidents that had occurred. 
Table 1: Selection of PSA experiences commonly reported by paediatric residents ${ }^{3}$

\begin{tabular}{|c|c|}
\hline Reported Practices & Associated potentially serious consequences \\
\hline $\begin{array}{l}\text { - Unmonitored PSA during Magnetic Resonance } \\
\text { Imaging (MRI) }\end{array}$ & $\begin{array}{l}\text { Not discovering in time potentially dangerous side } \\
\text { effects (respiratory depression, hypoxia or bradycar- } \\
\text { dia). }\end{array}$ \\
\hline $\begin{array}{l}\text { - No formal monitoring, observation or assessment } \\
\text { during recovery following the procedure }\end{array}$ & $\begin{array}{l}\text { - Not discovering in time potentially dangerous late side } \\
\text { effects. Immediately after the procedure procedural } \\
\text { stress falls away while sedative effect is still present. } \\
\text { This may cause suddenly and unexpectedly deep seda- } \\
\text { tion and loss of control on vital functions. }\end{array}$ \\
\hline $\begin{array}{l}\text { - Deeply sedated patients not accompanied by a } \\
\text { professional competent in airway management }\end{array}$ & $\begin{array}{l}\text { Not discovering and/or managing in time potentially } \\
\text { dangerous side effects. }\end{array}$ \\
\hline - Waking up or moving during MRI & $\begin{array}{l}\text { - Incomplete and/or low quality results, limiting diagnos- } \\
\text { tic accuracy. }\end{array}$ \\
\hline $\begin{array}{l}\text { - Being called for additional intravenous sedation in a } \\
\text { child sedated with chloral hydrate for MRI }\end{array}$ & $\begin{array}{l}\text { - Procedural delay. } \\
\text { Risk of oversedation and undesirable deep sedation, } \\
\text { associated with loss of control on vital functions. }\end{array}$ \\
\hline $\begin{array}{l}\text { - Combination of pre-procedural feeding, swaddling } \\
\text { and sedative drugs in infants undergoing MRI }\end{array}$ & - Risk of vomiting and aspiration \\
\hline $\begin{array}{l}\text { - Absence of age-specific resuscitation tools and } \\
\text { drugs }\end{array}$ & - Not being able to start rescue interventions instantly \\
\hline $\begin{array}{l}\text { - Forced restraint during endoscopy procedures or } \\
\text { oncology procedures (e.g. bone marrow puncture) } \\
\text { because of ineffective PSA (mostly midazolam only) }\end{array}$ & $\begin{array}{l}\text { - Extreme patient discomfort } \\
\text { - Preprocedural anxiety for new procedure } \\
\text { - Ineffective procedure }\end{array}$ \\
\hline $\begin{array}{l}\text { - Incomplete endoscopy procedures because of } \\
\text { ineffective PSA }\end{array}$ & $\begin{array}{l}\text { - Ineffective procedure leading to incomplete diagnosis } \\
\text { and/or need for repeated endoscopy }\end{array}$ \\
\hline $\begin{array}{l}\text { - Non application of topical anaesthesia (e.g. EMLA }{ }^{\circledast} \text { ) } \\
\text { in non-urgent vascular access }\end{array}$ & $\begin{array}{l}\text { - Patient discomfort } \\
\text { - Preprocedural anxiety for new procedure } \\
\text { - Ineffective procedure }\end{array}$ \\
\hline
\end{tabular}

\section{Authority driven search for authoritative guidelines}

Following the 2 fatal PSA related accidents in children, the Inspectorate for Public Health requested the Netherlands Society for Anaesthesiology and the Dutch Society for Paediatrics to design a new guideline on this topic and to assure its actual implementation in daily practice. A coordinating working group was set up, consisting of representatives from 21 professional organizations (see chapter 5). With support from the Institute for Quality in Health Care (CBO), they established new, evidence based guidelines for PSA. Three separate subgroups were formed, respectively focussing on guidelines for sedation in intensive care patients, PSA in adults and PSA in children. The overall guideline process was coordinated by professor J Knape. The Dutch Society for Paediatrics requested me to chair the subgroup on Paediatric PSA. In the country of the blind, it was easy for the 'one-retinal-cell-having' man to become "king"...

\footnotetext{
${ }^{3} 50$ random paediatric residents were interviewed informally during APLS courses and national conferences or symposia in The Netherlands between 2004 and 2006. Table 1 summarizes the most frequently reported experiences.
} 
Although PSA-related safety was considered the central focus of the assignment, the subgroup decided to define guidelines not only for optimally safe but also for optimally effective PSA in children. The guidelines were drawn up using the method of evidence-based guideline development (EBGD). This means that conclusions were drawn from existing scientific evidence, in addition to other practical considerations, in order to formulate recommendations for daily practice. In addition, the assignment included the design of an education plan and an implementation plan.

Between May 2006 and September 2007 relevant literature was searched and summarized. Results were discussed during multidisciplinary meetings in which both anaesthesiology professionals (i.e. anaesthesiologist and anaesthesiology staff) as well as non-anaesthesiology medical professionals (i.e. paediatricians, paediatric neurologists, paediatric radiologists, paediatric gastroenterologists, paediatric surgeons, paediatric oncologists, paediatric dentists, emergency physicians and paediatric nurses) were represented. Being a sample of the real world, the working group actually functioned as an experimental garden in which all aspects of the longstanding sedation paradox could be discussed, analyzed and tested against the published scientific evidence and PSA practices around the world. This resulted in three central concepts. At first, PSA should be always optimally safe ánd effective, meaning that existing unsafe or ineffective techniques, sedatives and circumstances need to be banned and that optimal transparency should be achieved. A second and absolutely crucial concept states that PSA related safety and effectiveness are determined by professional competence rather than by professional title.

Partly due to the valuable input by the Dutch Association for Children in Hospitals (representing sick children and their parents) and representatives from clinical educationalists a third concept was defined. Based on ethical and legal considerations, the definition of PSA related effectiveness should not be restricted to procedural success only, but should include optimal patient comfort and the maximal avoidance of procedural pain, anxiety and forced immobilization.

The final version of the guideline was completed in 2009 and contained two main chapters: a first one on general preconditions regarding safety, effectiveness and competences and a second one on procedure-specific guidelines. Between September 2009 and May 2010 a third chapter was added containing a plan for implementation. The guideline text has been peerreviewed by subsequent evaluation rounds in the several contributing professional societies and was finally approved in November 2010. Currently (December 2011) the guideline is still waiting final authorization by the Netherlands Society for Anaesthesiology and the Dutch Society for Paediatrics. There is, however good hope that authorization will be a fact by the date this thesis is defended in public.

\section{Research to see, search and solve: the aims and methodology of this thesis}

Despite the above-mentioned dramatic impressions of ineffective and unsafe PSA practices in Dutch paediatric practice, it was initially impossible to define the exact extent of the problem. The latter was, however, crucial in order to know the relevance of a new guideline and to find potential targets for future guideline implementation and projects for improvement. Objective data on the real safety and effectiveness of PSA were, however, impossible to collect, due to the fact that in The Netherlands, PSA is not a distinct medical competence or act, nor is it covered by a health insurance code or fee. The absence of a formal registration and the twilight zone posi- 
tioning of PSA activities made it impossible to have reliable data. At that point of the journey scientific research became involved.

The very first aim of this thesis was to define as accurately as possible the safety and effectiveness of the current PSA practices in Dutch paediatrics. Since 'paediatrics' as well as 'procedures' are highly comprehensive concepts, it was decided to limit our research to general paediatricians and to the most important procedures they have to arrange PSA for. Next, the concepts 'safety' and 'effectiveness' had to be operationalized. Therefore, an appropriate and meticulous methodology had to be designed and applied carefully. Simple questioning (a sample of) these professionals on the safety and effectiveness of their PSA practice was likely to be exposed to multiple possible confounders:

- The observation that PSA in this population clearly seemed to be a twilight-zone activity, charged with pejorative connotations like unsafety, ineffectiveness, non-transparency and incompetence, made it highly unlikely that direct questioning would yield a reliable outline of the reality. Paediatricians unaware of the potential unsafety of their daily PSA practice might underestimate the real risks. On the other hand, in the absence of a practicable alternative, paediatricians could feel the tendency to defend the practice they had adopted out of necessity (or obstinacy). Alternatively, those professionals desperately expecting a solution for their PSA-related problems from the anaesthesiology department, the hospital directors or the government, might overrate the extent of the real problems. Simply assessing expectations regarding PSA among general paediatricians was not expected to result in an objective description of the problem.

- Direct questioning on PSA-related incidents was likely to underestimate the real level of (un)safety. Given the low incidence rate of major complications, (although the incidence of minor to moderate incidents and near misses is considerable) most professionals were likely to have no personal experience with PSA-related accidents. ${ }^{7-12}$ Near-accidents certainly occur more frequently but their recognition requires full and complete monitoring and registration.

- Direct observation of PSA practices all over the Dutch paediatric departments was likely to be a suitable methodology but was practically unfeasible. Analyzing PSA-practice related behaviour and personal feelings in a large population of professionals by use of standard ergonomic, observational tools would have been very hard and time consuming. In addition, by the practical impossibility to apply really blinded methodology, the behaviour of an observed professional would be certainly influenced by the presence of an observer.

As a consequence, an indirect strategy had to be designed. Regarding PSA-related safety we decided to investigate the level of (non-)adherence to available safety guidelines, as it had been shown earlier that full adherence to safety guidelines on PSA is associated with an increase in patient safety. ${ }^{8,13}$ A postal questionnaire was sent to general paediatricians working in all hospitals in the Netherlands, as registered by the Dutch Society of Paediatrics. Definitions of PSA were provided in an introduction. Respondents were requested to focus on procedures requiring moderate or deep sedation. The questionnaire included questions on adherence to established safety standards on PSA. Standards were selected from international guidelines on PSA, published between 1998 and 2006, 5, 6, 14-18 and divided into 4 domains ('Pre-sedation Risk Assessment', 'Monitoring during PSA', 'Recovery after PSA' and 'Facilities and Competences for Emergencies and Rescue'). Each domain was operationalized into subdomains and, next, into items that successively stress a specific safety aspect during PSA. In order to reduce the desirability bias, participants were not informed that they were assessed on their level of adherence. Fur- 
thermore, all items regarding adherence were 'hidden' amongst other questions on personal PSA practice. Methodology and results are specified in detail in Chapter 2 (part 2.1).

In order to assess PSA-related effectiveness we focused on the two most important procedures general paediatricians had reported to arrange PSA for. Using a survey, we examined which PSA techniques and sedatives general paediatricians mostly used for Magnetic Resonance Imaging (MRI) and Gastro-Intestinal Endoscopy (GIE) in children. Reported PSA practices were compared with the results of a systematic review (SR) of the literature. By means of the SR, we tried to answer the clinical question as to which the safest and most effective technique for PSA during respectively MRI and GIE in childhood would be. (See Chapter 2, parts 2.2 and 2.3)

The second aim was to understand how paediatricians felt about their personal PSA practice within the above-mentioned setting of non-transparency, ineffectiveness and potential unsafety. A quality audit by the Dutch Society of Paediatrics had revealed that general paediatricians reported sedation failure, unsafety, lack of competence and especially a lack of anaesthetic support as highly relevant problems in their daily PSA practice. In order to understand fundamentally the issue of practicing within such a setting, it must be looked upon as a habit-like behaviour. Habit-like behaviour is not an isolated phenomenon but rather the result of latent and progressively developing subjective beliefs emerging from the interaction between (external) norms and own actions. An important general belief is the extent in which paediatricians are satisfied or dissatisfied with their own PSA performance seen in the light of fulfilment to the professional norms they have to adhere to. Measuring satisfaction and dissatisfaction is important: if professionals are dissatisfied with their performance, it surely will reflect itself in their actual adherence behaviour. There is reasonable evidence from literature that feelings of frustration, dissatisfaction with personal practice or general 'unwellness' have negative effects on workplace productivity and efficiency but also on quality of patient care and patient safety. ${ }^{19}$ Therefore, we intended to operationalize, measure and analyze (dis-)satisfaction with personal PSA practice in Dutch general pediatricians entrusted with PSA. By a more profound understanding of the sources and variations of their (dis)satisfaction we aimed to define more practical advice for the improvement of PSA practice in Dutch general paediatricians. (See Chapter 2, part 2.4)

A third aim was to elaborate more fundamentally two major principles of the new Dutch guideline on PSA in children, i.e. the importance of professional competence (and not 'professional title') to guarantee optimal safety and effectiveness (See Chapter 3); and the patient's rights to optimal patient comfort (See Chapter 4). Chapter 3 contains a systematic review investigating the scientific evidence that may answer the clinical question 'which skills and competence are imperative to assure optimal effectiveness and safety of PSA in children'. By doing so we tried to clarify scientifically the grey area between PSA intended for deep sedation and anaesthesia and to define guidelines for the use of potent anaesthetics (e.g. propofol) by nonanaesthesiologists.

In Chapter 4 we focus on legal and ethical considerations regarding the patient's rights to optimal comfort. This is important because the definition of 'optimal PSA effectiveness' must include a clear endpoint on which level of patient comfort is desirable. This endpoint will not only define whether PSA is indicated but also which PSA technique is advisable. Recent research has shown that patients who need very painful procedures or who are expected to suffer from substantial emotional distress (e.g. children $<6$ years) will need deep sedation in order to complete successfully the procedure and to avoid major psychological trauma to the child, the family and healthcare staff ${ }^{20}$ The use of benzodiazepines without analgesia to achieve amnesia for a painful procedure is contraindicated, usually unnecessary and therefore basically inexcusable. In addition, the application of forced immobilization and physical restraint is increasingly consid- 
ered as inhuman and unacceptable in non-lifesaving procedures. ${ }^{18,21}$ In children, procedurerelated discomfort will cause resistance, possibly leading to imminent procedural failure. Consequently, children are frequently physically restrained and/or immobilized in order to allow diagnostic or therapeutic procedures to be carried out. The following are important reasons to continue restraining and immobilizing children:

- To overcome the child's resistance in order to facilitate a necessary procedure, yet that is experienced as painful and/or frightening by the child,

- The partial or complete failure of a child to cooperate with procedures, due to a lack of understanding or lack of susceptibility to being distracted, making it impossible to give an explanation or to obtain informed consent for a necessary medical intervention to be performed.

- The unavailability of effective PSA facilities and condition for children who are scared of a painful and/or stressful procedure (for example, a blood test, the insertion of a drip, bladder catheterization, the replacement of a stomach tube, redressing small wounds).

- Insufficiently deep PSA for a painful or frightening procedure. Examples are: very painful punctures, such as bone marrow or lumbar punctures, large wound dressings, suturing wounds, reducing fractures, endoscopic examinations and certain types of radiological examination.

Although immobilizing and restraining children are procedures which are daily carried out in many hospitals, and professionals therefore treat them with a certain level of casualness, the use of these techniques raises important ethical and legal questions, which are further elaborated in Chapter 4.

A fourth aim was to translate the findings of both our epidemiological research among general paediatricians, as well as the results of the systematic reviews and the guideline developmental process into practical recommendations for the improvement of paediatric PSA in The Netherlands. This work is summarised in the general discussion part of this thesis (Chapter 6).

Finally, we actively sought for international collaboration with other professionals involved in the improvement of paediatric PSA. The PSA-related problems we mentioned earlier in this chapter are in no way typical for Dutch paediatrics. Very similar accidents have occurred and continue to occur in other western countries, not even to speak of developing countries. A lot of inspiration came from the United States. About a decade ago Coté's hallmark paper on PSA related accidents and unsafety triggered a nationwide process of quality improvement. ${ }^{22,}{ }^{23}$ Paediatric emergency physicians played a prominent role in this process. ${ }^{24}$ This resulted in hundreds of scientific publications and in the creation of the Society for Pediatric Sedation (www.pedsedation.org). Within this particular context, important names to mention are Baruch Krauss (Boston), Steven Green (Loma Linda), Joseph Cravero (Lebanon), Shobha Malviya (Michigan) and Keira Mason (Boston). In Europe a lot of work had been done by Mike Sury, paediatric anaesthesiologist in Great Ormond Street Hospital, London. He set up the annual European sedation course in London and has chaired the guideline workgroup that designed the new NICE guideline on procedural sedation in children (2011). ${ }^{25}$

The collaboration with these experts resulted in one publication ${ }^{26}$ and in a chapter on 'Paediatric Sedation: the European experience and approach' in the very first handbook on paediatric PSA: Keira P. Mason (ed.), Pediatric Sedation Outside of the Operating Room: A Multispecialty International Collaboration, DOI 10.1007/978-0-387-09714-5_17,@ Springer Science+Business Media, LLC 2011. This chapter is added as Appendix 1. 


\section{References}

1. Ljungman G, Gordh T, Sorensen S, Kreuger A. Pain in paediatric oncology: interviews with children, adolescents and their parents. Acta Paediatr. 1999;88(6):623-630.

2. Sury MR, Smith JH. Deep sedation and minimal anesthesia. Paediatric anaesthesia. 2008;18(1):18-24.

3. Knape J. Sedation and/or analgesia by non-anesthesiologists [Sedatie en/of Analgesie door nietanesthesiologen]. www.cbo.nl. www.cbo.nl. Published 1998.

4. American Academy of Pediatrics Committee on Drugs: Guidelines for monitoring and management of pediatric patients during and after sedation for diagnostic and therapeutic procedures. Pediatrics. 1992;89(6 Pt 1):1110-1115.

5. Guidelines for monitoring and management of pediatric patients during and after sedation for diagnostic and therapeutic procedures: addendum. Pediatrics. 2002;110(4):836-838.

6. Practice guidelines for sedation and analgesia by non-anesthesiologists. Anesthesiology. 2002;96(4): 1004-1017.

7. Cravero JP, Blike GT, Beach M, Gallagher SM, Hertzog JH, Havidich JE, et al. Incidence and nature of adverse events during pediatric sedation/anesthesia for procedures outside the operating room: report from the Pediatric Sedation Research Consortium. Pediatrics. 2006;118(3):1087-1096.

8. Hoffman GM, Nowakowski R, Troshynski TJ, Berens RJ, Weisman SJ. Risk reduction in pediatric procedural sedation by application of an American Academy of Pediatrics/American Society of Anesthesiologists process model. Pediatrics. 2002;109(2):236-243.

9. Malviya S, Voepel-Lewis T, Eldevik OP, Rockwell DT, Wong JH, Tait AR. Sedation and general anaesthesia in children undergoing MRI and CT: adverse events and outcomes. British journal of anaesthesia. 2000;84(6):743-748.

10. Malviya S, Voepel-Lewis T, Tait AR. Adverse events and risk factors associated with the sedation of children by nonanesthesiologists. Anesthesia and analgesia. 1997;85(6):1207-1213.

11. Maxwell LG, Tobias JD, Cravero JP, Malviya S. Adverse effects of sedatives in children. Expert opinion on drug safety. 2003;2(2):167-194.

12. Sanborn PA, Michna E, Zurakowski D, Burrows PE, Fontaine PJ, Connor L, et al. Adverse cardiovascular and respiratory events during sedation of pediatric patients for imaging examinations. Radiology. 2005;237(1):288-294.

13. Pitetti R, Davis PJ, Redlinger R, White J, Wiener E, Calhoun KH. Effect on hospital-wide sedation practices after implementation of the 2001 JCAHO procedural sedation and analgesia guidelines. Archives of pediatrics \& adolescent medicine. 2006;160(2):211-216.

14. Yldzdas D, Yapcoglu H, Ylmaz HL. The value of capnography during sedation or sedation/analgesia in pediatric minor procedures. Pediatric emergency care. 2004;20(3):162-165.

15. Guideline statement: management of procedure-related pain in children and adolescents. J Paediatr Child Health. 2006;42 Suppl 1:S1-29.

16. Cote CJ, Wilson S. Guidelines for monitoring and management of pediatric patients during and after sedation for diagnostic and therapeutic procedures: an update. Pediatrics. 2006;118(6):2587-2602.

17. Krauss B, Green SM. Procedural sedation and analgesia in children. Lancet. 2006;367(9512):766-780.

18. SIGN Guideline 58: Safe sedation of children undergoing diagnostic and therapeutic procedures http://www.sign.ac.uk/pdf/sign58.pdf. Published 2004.

19. Wallace JE, Lemaire JB, Ghali WA. Physician wellness: a missing quality indicator. Lancet. 2009;374(9702):1714-1721.

20. Cote CJ. Round and round we go: sedation -- what is it, who does it, and have we made things safer for children? Paediatric anaesthesia. 2008;18(1):3-8.

21. Folkes K. Is restraint a form of abuse? Paediatric nursing. 2005;17(6):41-44.

22. Cote CJ, Karl HW, Notterman DA, Weinberg JA, McCloskey C. Adverse sedation events in pediatrics: analysis of medications used for sedation. Pediatrics. 2000;106(4):633-644.

23. Cote CJ, Notterman DA, Karl HW, Weinberg JA, McCloskey C. Adverse sedation events in pediatrics: a critical incident analysis of contributing factors. Pediatrics. 2000;105(4 Pt 1):805-814. 
24. Krauss B, Green SM. Training and credentialing in procedural sedation and analgesia in children: lessons from the United States model. Paediatric anaesthesia. 2008;18(1):30-35.

25. Sury M, Bullock I, Rabar S, Demott K. Sedation for diagnostic and therapeutic procedures in children and young people: summary of NICE guidance. BMJ (Clinical research ed.341:c6819.

26. Leroy PL, Gorzeman MP, Sury MR. Procedural sedation and analgesia in children by nonanesthesiologists in an emergency department. Minerva pediatrica. 2009;61(2):193-215. 


\section{Part 1.2 Historical perspectives, overview and definitions}

Our greatest glory is not in never falling, but in rising every time we fall Confucius (551-479 BC)

Parts of this Chapter have been published in:

- Piet LM Leroy, Michiel P Gorzeman, Mike RJ Sury. Procedural Sedation and Analgesia in children by non-anaesthesiologists in an Emergency Department. Minerva Pediatrica 2009; 61(2): 193-215.

- Mike RJ Sury, Piet LJM Leroy. Paediatric Sedation: the European experience and approach. Chapter 17 in Keira P. Mason (ed.), Pediatric Sedation Outside of the Operating Room: A Multispecialty International Collaboration, DOI 10.1007/978-0-387-09714-5_17, (C Springer Science+Business Media, LLC 2011.

This review summarizes the history, definitions and current state of the art of paediatric PSA. Unreferenced statements are the author's personal opinions. Sedation and analgesia of the critically ill child, induction for intubation, palliative sedation and sedation for psychiatric emergencies are beyond the scope of this chapter. 


\section{General definition}

In modern paediatrics invasive diagnostic and therapeutic procedures in children are part of daily practice. Many of these procedures are painful and stressful or are impossible to perform without immobility. Despite local anaesthesia, reassurance and distraction techniques (young) children are often unable to undergo these procedures without being physically restrained. Therefore some form of sedation and/or analgesia is indicated. In many settings procedural sedation and analgesia (PSA) has become the standard of care for the management of acute procedural pain and anxiety.

PSA can be defined as the use of sedative, analgesic, and/or dissociative drugs to provide anxiolysis, analgesia, sedation, and motor control during painful or unpleasant diagnostic and therapeutic procedures. ${ }^{1}$

\section{Historical perspective}

PSA was 'invented' about 6 decades ago and has been applied ever since by a wide variety of non-anaesthesiology professionals in need for a sedative adjunct that enabled them to perform painful and/or stressful procedures in non-cooperative children. As a consequence, PSA covers a heterogeneous and large spectrum of drugs and techniques and involves a wide range of settings, sedation levels, efficacy and associated risks. This 'morbid growth' of all kind of PSA practices has one main historical reason: the initial lack of anaesthesiology involvement.

\section{Insufficient anaesthesia support and resources}

In the last decades there has been a rising demand for PSA and anaesthesiologists have been (and still are) often unable or unwilling to provide a timely service. ${ }^{2,3}$ In theory that is hardly surprising: anaesthesia has been developed originally for surgical operations whereas the development of services outside the operating theatre has been slow. Several reasons may explain this. Traditionally anaesthesia has facilitated surgeons with efficient operating lists. Paediatricians, in contrast, have not scheduled their cases in a similar fashion and have not always pressed their need for services. Consequently they have tried to manage on their own with the intention of giving themselves control and responsibility; this has had limited success.

Anaesthesiologists have been reluctant to help them because resources have not been vouchsafed and facilities may not have the standards of operating theatres - at least that was a common perception. There was also a fear of working unsupported at a site remote from other anaesthesia colleagues. Given these problems, paediatricians, had no choice but to cope with providing sedation on their own. Anaesthesiologists who could help offered anaesthesia, which was considered perhaps as unnecessary, out of proportion, associated with higher risk, or more expensive than sedation. Finally, there was an underlying view that once a service was given to paediatricians it would lead to a considerable increase in demand that would not be possible to satisfy - it was a "bottomless pit." A very important issue is the fact that significant shortages of anaesthesia manpower (both of medical and of non-medical medical personnel) exist in many European countries. ${ }^{4}$ 


\section{The invention of PSA drugs}

In the absence of an applicable alternative, non-anaesthesiology specialists involved in medical care for children have been providing sedative techniques for many decades. In order to achieve sedation they made use of (combinations of) the then available sedative drugs, which were particularly used for premedication in surgical operations.

The importance of pre-medication for paediatric patients undergoing surgical procedures dates to the classic work of Waters, who believed that children were as entitled as adults to have pre-medication. ${ }^{5,6} \mathrm{Up}$ to the sixties of the last century, the recommended pre-medication was morphine with scopolamine. Later, phenothiazines were believed to be useful as anaesthetic pre-medications because of their sedative and vasodilator effects. ${ }^{6}$ These observations led to the combination of a narcotic with a phenothiazine, which induced deep sedation (sleeping) in the child and allowed easy separation from his or her parents before administration of the anaesthetic. The narcotic provided a base of analgesia whereas the phenothiazine lowered the blood pressure and blunted the pressor-responses to surgical stimulation. The combination of meperidine (an opioid), promethazine (an antihistaminic) and chlorpromazine (a phenothiazine), commonly referred to as the MPC or lytic cocktail, has been used for many decades as a sedative and analgesic cocktail for paediatric patients. ${ }^{6}$ Although the combination of a narcotic with phenothiazine was first developed to provide pre-anaesthetic sedation for patients about to undergo general anaesthesia, its primary use in children has been outside of the operating room. In fact, the MPC combination can be regarded as the very first PSA drug used in children. It has been used since the fifties of the last century as a primary sedative for infants and young children undergoing radiologic procedures as well as a sedative/analgesic for invasive procedures (suturing, bone marrow aspirations, cardiac catheterization, and renal biopsy). Later on the PSA drug arsenal was expanded with new molecules like chloral hydrate, barbiturates (e.g. pentobarbital), benzodiazepines (e.g. diazepam and midazolam) and short-acting opioids (e.g. fentanyl).

Although many of these drugs are currently considered as 'classical PSA drugs', none of them had been specifically been designed for PSA in children. Furthermore, their introduction was not accompanied by pharmacological research. Quite soon the most important disadvantages of these (combination of) drugs became clear: the unpredictability of the effect, the unpredictable duration of action and the potentially life threatening side effects such as airway obstruction, respiratory depression and cardiovascular collapse. However, the end (i.e. the growing need for sufficient sedation to perform a procedure in a non-willing child) justified the means.

\section{From critical questions on PSA related safety to guideline development}

In contrast to anaesthesia, PSA has never been the core business or monopoly of one single professional group. Furthermore, a formal training in PSA was in fact non-existing until recently. Not surprisingly it didn't take long before non-anaesthesiologists performing PSA were criticized by anaesthesiologists for applying inconsistent and ineffective techniques and neglecting standard anaesthetic safety rules (e.g. pre-sedation assessment, monitoring of vital parameters and monitored recovery). ${ }^{2}$ There is, indeed, good evidence from the literature that there has been a substantial number of severe PSA-related accidents caused by unsafe practice. ${ }^{7,8}$ In particular, following sedation accidents in children undergoing dental procedures, the American Academy of Paediatrics (AAP) published a first guideline on this topic in $1985 .{ }^{9}$ This was the beginning of a 
long and still ongoing process of professional development, changing PSA from a twilight zone activity into the daylight clearness of a separate medical act requiring specific precautions, assessment, indications contra-indications, skills and competences. Updates of this first guideline were published in 1992, 2002 and 2006. ${ }^{10-12}$ Similar guidelines were published by the American Society of Anaesthesiologists (ASA, 2002), The American College of Emergency Physicians (2003, 2008), the Scottish Intercollegiate Guideline Network (SIGN, 2004), the Royal Australasian College of Physicians (2006), The European Board of Anaesthesiology and the British National Institute for Health and Clinical Excellence (NICE-2010). ${ }^{13-18}$. All these guidelines specify similar safety precautions that include (1) assessment of the sedation risk prior to PSA, (2) informed consent, (3) guidelines on proper fasting status, (4) appropriate monitoring, (5) recovery standards (6) professional competence, training and (7) imperative rescue facilities.

\section{Standards on PSA related effectiveness}

All published guidelines have patient safety as their main topic. Only quite recently policy statements on PSA and guideline updates started to expand their focus to the duty to deliver effective PSA. This 'effectiveness' should not only be regarded from a procedural point of view (i.e. guaranteeing predictable procedural success and timing) but also from a patient's perspective (i.e. achieving optimal procedural comfort). Therefore differentiation between painful and nonpainful procedures is indicated. ${ }^{14}$ There is a growing consensus that patients who need very painful procedures or who are expected to suffer from substantial emotional distress (e.g. children $<6$ years) will need deep sedation in order to complete successfully the procedure and to avoid major psychological trauma to the child, the family and healthcare staff ${ }^{19}$ The use of benzodiazepines without analgesia to achieve amnesia for a painful procedure is done on a wrong indication, usually unnecessary and inexcusable. In addition, the application of forced immobilization and physical restraint is increasingly considered as inhuman and unacceptable in nonlifesaving procedures ${ }^{14,20}$

\section{Outcome of PSA}

\section{Safety issues}

Many professionals link safety of PSA with the administered drug. In the literature many descriptive studies can be found claiming high safety of all kinds of PSA drugs (e.g. barbiturates, benzodiazepines, opioids, chloral hydrate, lytic cocktails, or mutual combinations of these) in a variety of settings but usually in a limited series of patients $(<1000)$. Given an a priori incidence of sedation related severe adverse events of about $1 / 10000$, most of these studies are insufficiently powered to prove such conclusion. ${ }^{21}$ Often these studies use vague definitions for the adverse reactions they report and consider the absence of directly life-threatening events as synonym for 'safe'. Establishing accurate adverse event and complications rates from the available literature has been difficult because of the difficulty in aggregating results from previous studies that have used varied terminology to describe the same adverse events and outcomes. Moreover the study setting is usually a strictly controlled, well-equipped and dedicated sedation unit or team, which differs appreciably from the common setting in many practices. Finally, well-designed reliable studies comparing safety of different standards of care are non-existent. These limita- 
tions prevent the establishment of a sufficiently large database on which evidence-based practice guidelines may be based. Recently a panel of paediatric sedation researchers and experts was assembled to develop consensus-based recommendations for standardizing procedural sedation and analgesia terminology and reporting of adverse events. ${ }^{22}$ This initiative is a very important first step to create a uniform reporting mechanism for future studies to facilitate the aggregation and comparison of results.

Although the safety profile and the margins of safety of PSA drugs are different, there exists a general agreement that PSA related safety is determined by the circumstances and professional skills rather than by specific pharmacologic characteristics. In 2000 Coté published his often-cited retrospective critical incident analysis of adverse sedation events in paediatrics reported to the American Food and Drug Administration between 1969 and 1996. 95 incidents were reported, 51 resulting in death, 9 in permanent neurological injury and 21 in prolonged hospitalisation. Significant contributing factors were (1) out of hospital settings, (2) inappropriate monitoring of physiologic parameters, (3) inadequate resuscitation skills, (4) inadequate presedation medical evaluation and (5) inadequate recovery procedures. No particular medication was associated with a higher risk except that long-acting drugs, overdosing and drug interaction (particularly when 3 or more drugs were used) were all associated with mortality. ${ }^{7,8}$ As a consequence anaesthesiologists, throughout the world, have been quick to state the problems of sedation by the untrained and have published guidelines to prevent disasters. Safety recommendations regarding PSA are mainly based on indirect evidence, like Coté's report, on "common sense" and on generally accepted safety rules for general anaesthesia. However, there exists reasonable evidence that the implementation of these guidelines leads to a safer PSA practice in children. ${ }^{23-25}$ This finding is in line with the observed reduction in adverse events during paediatric anaesthesia by the adoption of a uniform and systematic practice, including a risk assessment, monitoring, a monitored recovery period and immediate availability of tools, drugs and competences for patient resuscitation. ${ }^{26}$ However, despite the publication of PSA safety guidelines there is also evidence that practice is still unsafe in many settings. ${ }^{27}$

\section{Levels of sedation: definitions and remarks}

Ever since the first guideline on PSA was published, authors have linked the level of sedation with potential respiratory and cardiovascular side effects and by this with necessary safety precautions and monitoring. ${ }^{12}$ Consequently, levels of sedation have been defined. Four commonly used definitions are summarized in Table 1.

Initially, the rather inconsistent terminology 'conscious sedation' resulted in confusion. Conscious sedation was an accepted endpoint in the continuum of conscious level. Conscious (sedation), meaning able to respond to the spoken word, has been replaced by the term Sedation/Analgesia and more recently by moderate sedation because it does not assume consciousness but rather that the patient is easily roused - usually by communication but also by other similar appropriate light stimulus. ${ }^{19}$ The term conscious sedation, for children, is currently considered as an oxymoron because uncooperative children only tolerate procedures when they are unconscious. Nevertheless it remains commonly used ${ }^{28}$

Light sedation, formerly called anxiolysis, is typically the result of one standard dose of midazolam or by the breathing of nitrous oxide (inspired concentration up to $50 \%$ ). ${ }^{29}$ Higher doses, or other drugs, either alone or in combination, are likely to cause deeper levels of sedation in some patients. 
Table 1: Standard definitions of levels of sedation and significance for the respiratory and cardiovascular condition

\begin{tabular}{|c|c|c|}
\hline Terminology & Former terminology & Definition ${ }^{1,12}$ \\
\hline Light sedation & Anxiolysis & $\begin{array}{l}\text { A drug-induced state during which patients respond normally to } \\
\text { verbal commands; although cognitive function and coordination may } \\
\text { be impaired, ventilatory and cardiovascular functions are unaffected. }\end{array}$ \\
\hline Moderate sedation & $\begin{array}{l}\text { Conscious sedation }^{9} \text {, } \\
\text { Sedation/analgesia }^{11,13}\end{array}$ & $\begin{array}{l}\text { A drug-induced depression of consciousness during which patients } \\
\text { respond purposefully to verbal commands (e.g., "open your eyes," } \\
\text { either alone or accompanied by light tactile stimulation, such as a } \\
\text { light tap on the shoulder or face, not a sternal rub). For older pa- } \\
\text { tients, this level of sedation implies an interactive state; for younger } \\
\text { patients, age appropriate behaviours (e.g., crying) occur and are } \\
\text { expected. Reflex withdrawal, although a normal response to a } \\
\text { painful stimulus, is not considered as the only age-appropriate } \\
\text { purposeful response (i.e., it must be accompanied by another } \\
\text { response, such as pushing away the painful stimulus, to confirm a } \\
\text { higher cognitive function). }\end{array}$ \\
\hline
\end{tabular}

Deep sedation

Anaesthesia
In a state of moderate sedation, no intervention is required to maintain a patent airway, and spontaneous ventilation is adequate. Cardiovascular function is usually maintained. However, in the case of procedures that may themselves cause airway obstruction (e.g., dental or endoscopic), the practitioner must recognize an obstruction and assist the patient in opening the airway. If the patient is not making spontaneous efforts to open their airway to relieve the obstruction, then the patient should be considered to be deeply sedated.

A drug-induced depression of consciousness during which patients cannot be easily aroused but respond purposefully (see discussion of reflex withdrawal above) after repeated loud verbal or painful stimulation (eg, purposefully pushing away the noxious stimuli).

The ability to independently maintain ventilatory function may be impaired. Patients may require assistance in maintaining a patent airway, and spontaneous ventilation may be inadequate. Cardiovascular function is usually maintained. A state of deep sedation may be accompanied by partial or complete loss of protective airway reflexes.

A drug-induced loss of consciousness during which patients are not arousable, even by painful stimulation.

The ability to independently maintain ventilatory function is often impaired. Patients often require assistance in maintaining a patent airway, and positive-pressure ventilation may be required because of depressed spontaneous ventilation or drug-induced depression of neuromuscular function. Cardiovascular function may be impaired. 
Moderate sedation is often used incorrectly to describe a state that is probably more like deep sedation: reflex withdrawal to a painful stimulus alone should not be considered as rousable. ${ }^{19}$

The term deep sedation has been under discussion in some professional groups, because it may be indistinguishable from anaesthesia. While this point may be overstated it has led to the recommendation that both deep sedation and anaesthesia must be managed by the same personnel, equipment and facilities. The definition becomes more useful as a description of the intended conscious level rather than as a division on the basis of resources or risks. In a similar desire, two other descriptions of deep sedation/anaesthesia have been used: light anaesthesia or minimal anaesthesia are terms that could be used to describe a technique in which the patient seems unconscious although any appreciable stimulation is likely to rouse them. ${ }^{30,31}$

Dissociative sedation is not a term in common use but generally it is understood. This type of sedation caused by ketamine has also been called a trance-like cataleptic state. ${ }^{32}$ Ketamine sedation or anaesthesia may be more appropriate terms. ${ }^{32}$ Indeed, ketamine causes a condition of deep sedation or anaesthesia (i.e. patient cannot easily be aroused), while protective airway reflexes, spontaneous respiration and cardiovascular stability are generally maintained, while offering substantial analgesia. Ketamine is unique in this respect.

Except for light sedation/anxiolysis and dissociative sedation, the question remains to what extent the outcome level can be predicted, especially when non-titratable drugs are used. This question is relevant since PSA by non-anaesthesiologists who have not been trained in providing controlled sedation, is often performed using long- and slow-acting medications. The main reason for this is their supposedly wide safety margin (i.e. the difference between a safe and unsafe dose is large). Commonly used sedation drugs, such as oral Chloral hydrate, intravenous midazolam+fentanyl or meperidine, or barbiturates, cause wide variations in depth of sedation. If a single dose is given the goal of either conscious or deep sedation is not achieved in a substantial number of children and, for individual cases, prediction of the sedation end point is unreliable. ${ }^{33}$ Considering sedation levels as a sliding scale, rather than a step-by-step change in consciousness, the transition from one level to another can be subtle, sudden and unexpected. It is, therefore, advisable to apply the same safety and monitoring precautions for all levels of sedation beyond light sedation.

\section{Effectiveness of PSA}

From a service-driven point of view, a major goal of PSA is to make a given procedure feasible in a child in cases when this is not expected to happen in a fully conscious child. In many studies effectiveness is assessed by measuring two outcomes. The easiest is completion of the procedure and the more difficult is patient, parent or clinician satisfaction. However, there exist no validated outcome parameters on effectiveness of PSA, making comparison between different regimes and studies difficult. In his review article Cravero explained that a child who is given a dose of oral Midazolam and cries or screams during a lumbar puncture and then sleeps for $2 \mathrm{~h}$ is often considered an equal success as the patient who lies perfectly still under brief propofol sedation, although objective observers would clearly count the second strategy a success and the first a failure. ${ }^{21}$ Several reports prove that procedural pain management is still often inadequate, or that available techniques are not applied, leading to procedural failure and avoidable procedural pain. ${ }^{34-37}$ Currently there is an increased awareness of the importance of providing adequate analgesia and anxiolysis in children. It has been shown that repeated procedural pain and anxiety in childhood may carry significant morbidity. ${ }^{38,39}$ 
Therefore, in evaluating the success of a PSA drug or technique, at least two other major outcome measures must be considered. Firstly, it must be questioned whether patient's comfort is maximised. Like adults, children have every right to optimal health care. This implies that whenever distraction techniques, local anaesthesia or minimal sedation fail to keep the child comfortable, more effective sedation should be deployed. In young children under 6 years, deep sedation is often indicated. ${ }^{12,19,40}$ Unfortunately physical restraint is commonly used nowadays for imperative procedures in young children. In most European civil codes young children have not or only partially the right to refuse a medical treatment that has been consented by a parent or a legal guardian. This means that, if a child refuses to cooperate, current healthcare legislation permits professionals, after parental consent, to use restraint in order to allow a certain procedure to be executed, even if the resistance of the child clearly suggests disapproval. Despite this legal framework, many people are unhappy with using restraint and it has been questioned whether restraint is a form of abuse. ${ }^{20}$ Several authors have postulated that procedural restraint may be contrary to the Human rights act and the United Nations Convention on The Rights of the Child. ${ }^{20,41}$ Moreover, since knowledge and technology exist to provide PSA easily, safely and painlessly for most procedures, restraining a terrified and resisting child for a painful procedure cannot be simply justified. ${ }^{42}$ Finally, procedural comfort needs more than effective PSA only. Timely application of effective methods for local anaesthesia, use of behavioural and distraction techniques and the availability of rescue anaesthesia are currently considered as essential parts of an "ouchless" concept and a comfort-centred care for sick children. ${ }^{43}$

Secondly, organizational aspects and cost-effectiveness have to be taken into account. Ideally a PSA technique brings a child to an effective level of sedation just before the start of the procedure and recovery (wake up) shortly after its termination. A PSA technique that matches appropriately the length of sedation to the requirements of a given procedure is clearly superior to less controlled or less titratable techniques. For example, deep sedation by titration of intravenous midazolam+fentanyl is, in comparison with Propofol, time-consuming, may have an inadequate effect and needs longer and more personnel-intensive recovery of between 1 to 2 hours. ${ }^{40}$ Both regimens have similar incidences of adverse events, although the midazolam+fentanyl combination is often considered as safe in non-anaesthetic hands, while professional barriers exist for the use of Propofol by non-anaesthetists. For many brief procedures in paediatrics (e.g. emergency CT imaging) long-acting enteral drugs (e.g. chloral hydrate) or intramuscular drugs (e.g. pentobarbital, meperidine) are used. These drugs cause an unpredictable type of sedation, regarding both the expected time of effective sedation and the level of sedation. Furthermore they may cause prolonged sedation for several hours which is inappropriate for a procedure that takes no more than a few minutes. ${ }^{44}$ Although these techniques are often catalogued as highly successful (successful termination in about 70-90\%), they are far less effective than techniques using ultrashort-acting agents that can be administered to match PSA with the needs of the procedure. For example, if IV access is established, the fast action of propofol as a basic sedative can achieve deep sedation or anaesthesia in 60 seconds and recovery time of less than 10 minutes even for prolonged painless procedures. Painful procedures can be made less painful by the use of an appropriate opioid or local anaesthetic (applied while the child is sedated with propofol).

In conclusion, each PSA should be directed ideally to a specifically determined sedation level and this makes the use of short acting and "titratable" drugs (e.g. propofol) advantageous over and thus preferable for the use of long acting drugs. Short acting drugs can be used to overcome the pain and distress that varies according to the procedures and the patients themselves. 


\section{Central themes of recent international safety guidelines on PSA}

\section{Informed Consent}

PSA must be considered as a separate medical act and not as a part of the actual procedure. Therefore patients (or their legal guardians) should be informed of and agree to the administration of sedation/analgesia, including its benefits, risks, and limitations, as well as possible alternatives. ${ }^{12}$ According to article 5 of the charter of The European Association for Children in Hospital (EACH) on children's rights before, during or after a stay in a hospital, children and parents have the right to informed participation in all decisions involving their health care. ${ }^{45}$ Specific regulations on informed consent may vary with local legislation. In many countries informed consent should be obtained verbally and be written down in the patient's file. The use of information booklets or handouts should be considered and promoted.

\section{Patient selection and risk assessment}

Sedation may impair vital reflexes and functions. Patients with certain pre-existing conditions or diseases (potentially) affecting respiration, circulation or consciousness may be more vulnerable to sedation induced adverse events and complications. It has been advised to use the ASA physical status classification (Table 2) to judge the patients' overall sedation risk by assessing the type and severity of underlying medical problems. ${ }^{1,13}$ Patients with an ASA classification of I or II are generally considered eligible for PSA by non-anaesthesiologists; children in ASA class III or more low level consultation of a (paediatric) anaesthesiologist should be carried out to establish the most appropriate sedation policy. In many of these cases anaesthesiologist involvement will be required.

Age, developmental age, mental state, communication problems, uncontrolled movements and hypotonia are not easily classified within the ASA classification even though they are all associated with higher rates of adverse events and sedation failure. Sedation in children less than 1 year has been associated with an increased risk. Also in children older than 6 years an increased risk exists, probably associated with increased anxiety levels in this age group and therefore with a higher tendency to drug overdosing. ${ }^{44,46}$ In these conditions only well-trained professionals with appropriate experience should perform PSA. In many cases this will be a (paediatric) anaesthesiologist, except when a non-anaesthesiologist professional who is specifically trained for paediatric PSA is readily available. Routine assessment must include current medication use, drug allergies or intolerances, and previous experiences with anaesthesia or PSA. Focussed assessment should be directed to the airway including history of apnoea or obstruction and, inspection for abnormalities that might impair airway patency (e.g. retrognathia, large adenoid tissue or tonsils, obesity, limit neck mobility). Careful assessment of respiratory and circulatory condition is as important. 
Table 2: American Society of Anaesthesiologists (ASA) Physical status classification for the assessment of PSA related risks in children.

\begin{tabular}{|c|c|c|c|}
\hline Class & Definition & Examples & Suitability for PSA \\
\hline 1 & Healthy child & $\begin{array}{l}\text { No particular medical history } \\
\text { No maintenance medical treatment }\end{array}$ & - Excellent \\
\hline 2 & $\begin{array}{l}\text { Child with } \\
\text { mild systemic } \\
\text { disease, but } \\
\text { no functional } \\
\text { limitations }\end{array}$ & $\begin{array}{l}\text { Mild asthma without respiratory distress or need for oxygen } \\
\text { Viral febrile disease (e.g. common cold) without respiratory com- } \\
\text { promise } \\
\text { Controlled seizure disorder (no recent seizures) } \\
\text { Corrected cor vitium with normal cardiac function, no pulmonary } \\
\text { hypertension or respiratory compromise } \\
\text { Anaemia without circulatory symptoms } \\
\text { Controlled diabetes mellitus } \\
\text { Pleural empyema without respiratory compromise } \\
\text { Malignancy without respiratory, neurological or circulatory com- } \\
\text { promise }\end{array}$ & - Generally good \\
\hline 3 & $\begin{array}{l}\text { Child with } \\
\text { severe sys- } \\
\text { temic disease } \\
\text { with func- } \\
\text { tional limita- } \\
\text { tions }\end{array}$ & $\begin{array}{l}\text { Moderate to severe asthma with moderate respiratory distress or } \\
\text { need for oxygen } \\
\text { Broncho pulmonary dysplasia with low need for oxygen } \\
\text { Pneumonia } \\
\text { Poorly controlled seizure disorder } \\
\text { Corrected cor vitium with normal cardiac function, no pulmonary } \\
\text { hypertension or respiratory compromise } \\
\text { Anaemia with circulatory symptoms } \\
\text { Poorly controlled diabetes mellitus; moderate diabetic keto- } \\
\text { acidosis } \\
\text { Pleural empyema with moderate respiratory compromise } \\
\text { Malignancy with moderate respiratory, neurological or circulatory } \\
\text { compromise } \\
\text { Moderate obesity } \\
\text { Obstructive sleep apnoea syndrome }\end{array}$ & $\begin{array}{l}\text { - Usually poor } \\
\text { - Consider procedural } \\
\text { benefits and seda- } \\
\text { tion risks } \\
\text { - Assess competency } \\
\text { of sedation service } \\
\text { - Seek advice from a } \\
\text { paediatric anaesthe- } \\
\text { siologist }\end{array}$ \\
\hline 4 & $\begin{array}{l}\text { Child with } \\
\text { severe sys- } \\
\text { temic disease } \\
\text { with constant } \\
\text { threat of life. }\end{array}$ & $\begin{array}{l}\text { Life threatening asthma, pneumonia, ARDS } \\
\text { Severe broncho pulmonary dysplasia } \\
\text { Sepsis } \\
\text { Severe diabetic keto-acidosis } \\
\text { Any disease with advanced degrees of pulmonary, cardiac hepatic, } \\
\text { renal or endocrine insufficiency. }\end{array}$ & $\begin{array}{l}\text { - Poor } \\
\text { - Seek advice from a } \\
\text { paediatric anaesthe- } \\
\text { siologist }\end{array}$ \\
\hline 5 & $\begin{array}{l}\text { Moribund } \\
\text { child which is } \\
\text { not expected } \\
\text { to survive } \\
\text { without the } \\
\text { procedure }\end{array}$ & $\begin{array}{l}\text { Septic shock } \\
\text { Hypovolemic hemorrhagic shock } \\
\text { Neurotrauma }\end{array}$ & - Extremely poor \\
\hline
\end{tabular}




\section{Fasting guidelines}

Guidelines state that the ASA fasting guidelines for children receiving general anaesthesia should be applied to children undergoing elective PSA. ${ }^{1,12,14}$ However, especially in the setting of an Emergency Department (ED), this recommendation is often hard to achieve. Furthermore, several well-designed studies in children undergoing PSA in an ED could not find a clear relationship between the pre-procedural fasting status and the incidence of clinically important complications. ${ }^{16,47-52}$ Moreover prolonged fasting, resulting in increased unrest and anxiety levels, has been linked with sedation failure ${ }^{24,50,53}$ and even with an increased risk of vomiting. ${ }^{49}$

A recent systematic review recommends that PSA may be safely administered to paediatric patients in the ED who have had recent oral intake. ${ }^{16}$ Nevertheless it may be wise to balance any potential risk for vomiting and pulmonary aspiration with the desirable timing of a procedure and the required depth of sedation. Risks and benefits must be weighed for each patient. ${ }^{1,16}$ Green et al. tried to translate the available evidence in a consensus-based clinical practice advisory. It should be used to form the base for local protocols on this subject. ${ }^{54}$

\section{Monitoring}

Monitoring is intended to detect changes that could prevent PSA-related complications. Respiratory failure and hypoxia due to airway obstruction or hypoventilation are the most frequently reported severe complications during PSA. ${ }^{46,55,56}$ They are mostly related to a deep level of sedation. ${ }^{8}$ Bradycardia and cardiac arrest during PSA have been reported infrequently and are mostly caused by preceding respiratory failure. As a consequence, guidelines on PSA state specifically that monitoring of (1) oxygen saturation, (2) breathing parameters, (3) heart rate, (4) blood pressure and (5) level of consciousness are essential during PSA especially when moderate and deep levels of sedation are intended or can be expected. ${ }^{12}$ For light sedation pulse oximetry is usually considered sufficient. Nitrous oxide alone in oxygen (maximum inspired concentration $50 \%)$ may not need any monitoring provided that verbal interaction with the patient is maintained. ${ }^{28}$ However, oximetry is recommended, also because of the ease of saturation monitoring and the wide availability of these devices. Irrespective of the intended level of sedation, monitoring should not be restricted to the procedure time but should start prior to the administration of sedative drugs in order to be informed about patient's basic condition. Subsequently it should be continued until full recovery.

In spite of these recommendations, no studies have been published comparing the outcome of different levels of monitoring, although in cases of lack of evidence common sense must prevail in decision making. Published guidelines recommend blood pressure (BP) monitoring during moderate or deep sedation, when verbal communication is impossible and during lengthy procedures. ${ }^{12,14}$ However, no study has shown any benefit of BP measurements during PSA. Furthermore, in a state of moderate sedation for non-painful procedures (e.g. imaging), BP monitoring might wake-up the patient and may be counter-productive. In case drugs are used with an expected effect on blood pressure (e.g. hypertension in ketamine and hypotension in propofol), it seems reasonable to apply BP monitoring. However, neither drug induced hypotension nor hypertension are likely to cause harm in ASA I or II patients. ${ }^{57}$ Cardiovascular compromise due to hypoxia is a late sign of PSA induced respiratory failure that should have been discovered by appropriate visual and technical respiratory monitoring before hypotension arises. 
Pre-sedation BP monitoring should be used in any child who may be vulnerable to hypotension caused by hypovolaemia, dehydration or sepsis.

Capnography is probably infrequently used for sedation in Europe. This is due to technical problems of sampling expiratory gases in non-intubated patients. Nevertheless recent research illustrates their usefulness for monitoring during PSA. Capnography is the non-invasive measurement of the partial pressure of carbon dioxide in the expired air. This $\mathrm{CO}_{2}$ monitor displays an end-tidal $\mathrm{pCO}_{2}\left(\mathrm{ETCO}_{2}\right)$ level as well as an expiratory waveform. In anaesthesia capnography is a compulsory standard of care for respiratory monitoring. It provides continuous breath-by-breath information confirming airway patency, adequacy of ventilation and cardiac output (in particular, in intubated patients it confirms tracheal tube placement and adequacy of tidal volume).

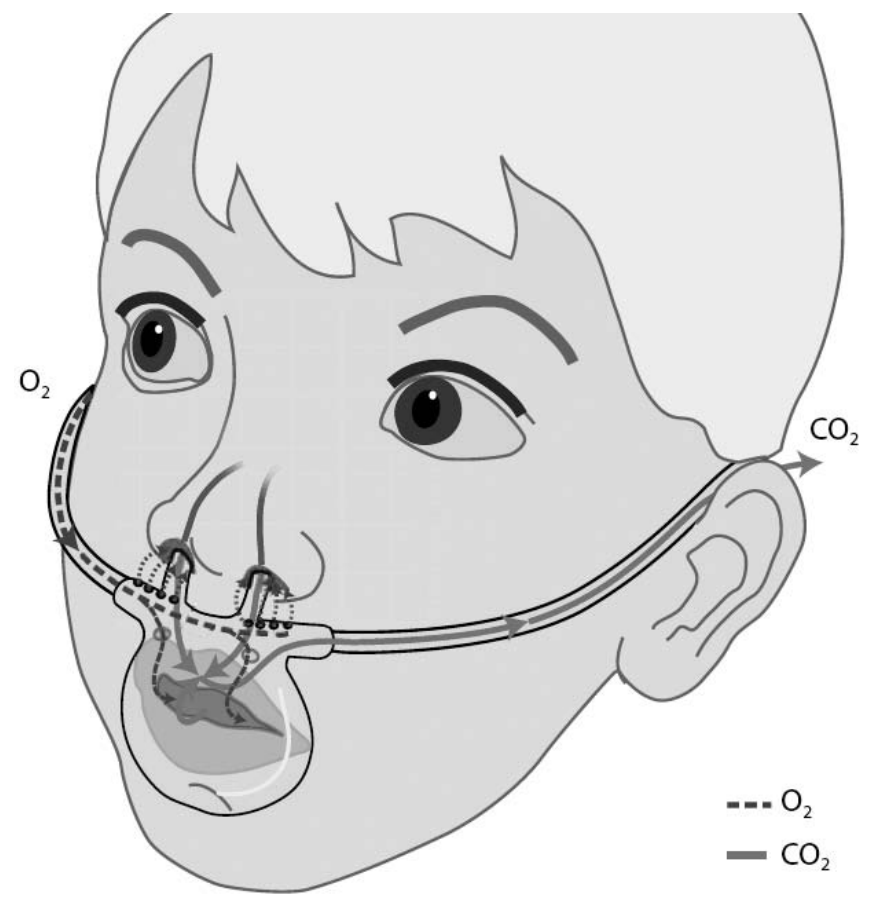

Figure 1: Capnography: exhaled air is sampled via the nasal cannula (breathing through the nose) and via a sampling piece that dangles in front of the mouth (breathing through the mouth). The amount of $\mathrm{CO}_{2}$ in the exhaled air is measured. If necessary also oxygen can be administered. (Smart Capnoline ${ }^{\circledR}$ - Oridion )

Capnography is much less applied for the ventilatory assessment in spontaneously breathing sedated patients. In the recently updated AAP guideline on PSA capnography is mentioned as a valuable monitor to detect the presence or absence of respirations, airway obstruction, or respiratory depression. The use of capnography is especially encouraged for sedated children, particularly in those sedated in less-accessible locations where visually assessment of ventilation is limited (e.g. MRI or CT imaging in darkened rooms) or if the face is hidden under sterile covers. ${ }^{12}$ Capnography can identify respiratory depression and airway complications before clinical observation and, crucially, significantly earlier than pulse oximetry. ${ }^{58-64}$ For early detection of major airway obstructions and profound hypoventilation, capnography is the most reliable clinical monitor available. However the exact understanding and clinical interpretation of moderate 
changes in $\mathrm{ETCO}_{2}$ levels during PSA are less clear. Currently new nasal cannulae are available for simultaneous oxygen delivery and microstream capnography. (figure 1) Krauss and Hess have recently reviewed the physiology, the interpretation of the $\mathrm{CO}_{2}$ waveform and the recognition of normal, abnormal and sedation induced ventilatory patterns. ${ }^{65}$

Level of consciousness monitoring could be useful, is mandatory, but is rarely applied in PSA. Since deep sedation is associated with unexpected adverse effects, regular assessment of conscious level during sedation and in the subsequent recovery phase must be a routine. ${ }^{11,12}$ Monitoring the level of consciousness can be achieved by both observational as well as objective technical tools. Several validated observational scoring systems for consciousness assessment are available ${ }^{66-68}$ although they are limited in distinguishing moderate from deep sedation. It has been shown, at least for chloral hydrate sedation, that combining the University of Michigan Sedation Scale (UMSS-Table 3) and the Modified Maintenance of Wakefulness Test (MMWT) is more reliable to describe the condition 'fully awake after PSA' compared with nursing judgment using standard criteria. ${ }^{69}$

Table 3: University of Michigan Sedation Scale ${ }^{69}$

\begin{tabular}{ll}
\hline Score & Descriptors \\
\hline 0 & Awake and alert \\
1 & Minimally sedated: tired/sleepy, appropriate response to verbal conversation and/or sound \\
2 & $\begin{array}{l}\text { Moderately sedated: somnolent/sleeping. Easily aroused with light tactile stimulation or a simple verbal } \\
\text { command }\end{array}$ \\
3 & Deeply sedated: deep sleep, arousable only with significant stimulation \\
4 & Unarousable \\
\hline
\end{tabular}

Bispectral Index System (BIS) monitoring is an electro-encephalogram based monitoring device that is validated for the objective measurement of consciousness in adult patients undergoing general anaesthesia. Several reports have suggested that BIS may be a useful tool for objective assessment of depth of sedation on the Paediatric Intensive Care Unit (PICU). ${ }^{70-72}$ In propofol based PSA it has been shown that BIS could be a useful monitoring guide for drug titration in children. ${ }^{73}$ BIS is less reliable in the differentiation between moderate and deep sedation and reliability is also age-and drug-dependent. BIS should be interpreted cautiously in children sedated with ketamine, midazolam, chloral hydrate or opioids. ${ }^{74}$

\section{Preventive supplemental oxygen therapy}

Hypoxia is by far the most frequently observed complication during PSA and is mainly the result of drug induced hypoventilation, apnoea or airway obstruction occurring shortly after administration of the initial dose. Combination of sedative and analgesic drugs (e.g. propofol and midazolam+fentanyl) cause more (mostly transient) hypoxia periods than others (e.g. midazolam and ketamine). ${ }^{17}$ The American Society of Anaesthesiologists recommends the use of supplemental oxygen for patients undergoing deep sedation and suggests considering its use during moderate sedation. ${ }^{13}$ In adults low-flow oxygen (up to $31 / \mathrm{min}$ ) did not reduce significantly the risk of hypoxia during deep sedation with propofol or moderate sedation with midazolam+fentanyl. ${ }^{75,76}$ The benefit of supplemental oxygen during PSA in children has not been studied. ${ }^{77}$ Theoretically, preventive oxygen may prevent desaturation or may simply delay the onset, especially in cases where hypoventilation or airway obstruction goes unnoticed initially. Oxygen masks or nasal 
cannulae can be impractical in anxious and uncooperative children, causing unnecessary stress. Furthermore, in pre-oxygenated children it may take up to 4 minutes before a persisting apnoea causes desaturation below $90 \%{ }^{78,79}$ Professionals relying on a pulse oximetry for respiratory monitoring may overlook episodes of hypoventilation, especially when their patients are given supplemental oxygen. The simultaneous delivery of oxygen and microstream capnography by specifically designed nasal cannulae might be the best practical solution. Capnography frequently identifies respiratory depression undetected by the treating physicians, at least far earlier than pulse oximetry does (See above).

\section{Recovery and discharge from monitoring}

Whatever the half-life of the sedative drug, patients will not return instantly to their baseline condition once the procedure has been terminated. Occasionally a child can recover and then become resedated later. Resedation may occur where the balance between a painful procedure and sedation suddenly changes as a consequence of ending the procedure resulting in a decrease of adrenergic stimulation. ${ }^{8}$ In a study of 1341 PSA sessions in children in an ED, about $8 \%$ of 184 adverse events occurred after the actual procedure. Serious adverse events occurred approximately 2 minutes (median time) after the final medication dose but did also occasionally reappear 25 minutes later. ${ }^{80}$ Therefore a PSA with moderate or deep sedation should always be followed by a monitored recovery period, including (1) the regular direct observation and assessment of the vital functions (i.e. respiration and circulation) and the level of consciousness, (2) the continuous monitoring of oxygen saturation, respiration and heart rate as long as the patient is not fully alert and (3) the use of specific criteria for defining when the "patient is fully awake" and the decision of when "patient can be discharged from monitoring". These data should be recorded. Several scores to assess recovery have been validated. ${ }^{66,68,69}$ It has been recommended to use predetermined discharge criteria. ${ }^{12}$ An interesting method to determine return to full alertness is the Modified Maintenance of Wakefulness Test (MMWT) which tests the ability of a child to remain awake for at least 20 minutes while being in a quiet environment. ${ }^{69}$ Ideally, children who underwent PSA should be closely observed and monitored in a quiet recovery room in the presence of a professional trained in the assessment of age-specific vital parameters and consciousness.

Using a telephone questionnaire 24 hours after PSA, Steurer studied the incidence of adverse events after discharge from the ED. At least one adverse effect was reported in $42 \%$ of respondents, including lethargy (12\%), vomiting (7\%), behavioural changes $(7 \%)$, headache $(6 \%)$, balance/gait disturbances $(5 \%)$, nausea (4\%), sleep disturbances $(4 \%)$, nightmares (4\%), hallucinations $(2 \%)$, and ear pain $(0.2 \%)$. Anticipatory guidance about possible late adverse effects related to PSA should be given to parents and caregivers prior to discharge. ${ }^{81}$

\section{Rescue tools and skills}

Although the relative risk for severe adverse events is low, insufficient resuscitation resources or skills have been associated with fatal outcome of PSA related accidents. ${ }^{8}$ Age-specific equipment for advanced paediatric life support (APLS) must be immediately available wherever PSA is delivered, especially when moderate or deep levels of sedation are intended. (Table 4) For predictable light sedation (i.e. nitrous oxide alone) the presence of basic life support (BLS) facilities and skills is considered adequate. 
Table 4: Drugs and equipment to rescue a sedated child

\begin{tabular}{|c|c|}
\hline \multirow[t]{9}{*}{ Airway } & Immediately available/ready to use \\
\hline & Oropharyngeal airways (age appropriate) \\
\hline & Laryngeal masks (age appropriate) \\
\hline & Suction unit + appropriate suction catheters \\
\hline & Quickly available \\
\hline & Nasopharyngeal airway \\
\hline & Laryngoscope + age-appropriate blades \\
\hline & Endotracheal tubes (age appropriate) \\
\hline & Stylettes \\
\hline \multirow[t]{3}{*}{ Oxygen } & Immediately available/ready to use \\
\hline & High-flow oxygen \\
\hline & Non-rebreathing masks \\
\hline \multirow[t]{4}{*}{ Breathing } & Immediately available/ready to use \\
\hline & Face masks (age appropriate) \\
\hline & Self-inflating ventilation bag \\
\hline & Capnometry \\
\hline \multirow[t]{9}{*}{ Circulation } & Immediately available/ready to use \\
\hline & IV catheters (age appropriate) \\
\hline & Fixation tape \\
\hline & Intra osseous needle \\
\hline & Syringes (including 50-60 ml syringes for IV fluid loading) \\
\hline & Quickly available \\
\hline & 3-way stopcocks \\
\hline & IV tubing \\
\hline & Defibrillator \\
\hline \multirow[t]{9}{*}{ Drugs } & Immediately available/ready to use \\
\hline & Adrenaline (epinephrine) \\
\hline & Atropine \\
\hline & Glucose $10 \%$ \\
\hline & IV fluid (crystalloid) \\
\hline & Flumazenil \\
\hline & Naloxone \\
\hline & Quickly available \\
\hline & Medication for intubation \\
\hline
\end{tabular}

\section{Recent developments}

More recently, and mainly due to the limited effectiveness and predictability of the classical PSA drugs, also typical anaesthetics like ketamine and propofol have been applied for PSA in spontaneously breathing children. ${ }^{40,82}$ In addition, in many countries a clear trend is seen to entrust deep sedation, including PSA based on anaesthetics, to specifically trained non-anaesthesia professionals because of the scarcity of paediatric anaesthesiologists. Emergency physicians, intensivists and gastro-enterologists have been prominent in this development. ${ }^{23,40,83,84}$ There is a growing evidence, also in children, that well-trained non-anesthesiologists may provide propofol sedation safely. ${ }^{85}$ It has also been shown that within the setting of adequate training and 
strict safety measures no differences exists in rates of major complications among different specialist (i.e. anesthesiologists and non-anesthesiologists). ${ }^{86}$ Appropriate safety precautions, monitoring and professional skills, rather than professional title, are determinants for the safe and effective use of propofol for PSA. ${ }^{40}$ 


\section{References}

1. Krauss B, Green SM. Procedural sedation and analgesia in children. Lancet. 2006;367(9512):766-780.

2. Ratnapalan S, Schneeweiss S. Guidelines to practice: the process of planning and implementing a pediatric sedation program. Pediatric emergency care. 2007;23(4):262-266.

3. Cote CJ. Discharge criteria for children sedated by nonanesthesiologists: is "safe" really safe enough? Anesthesiology. 2004;100(2):207-209.

4. Egger Halbeis CB, Cvachovec K, Scherpereel P, Mellin-Olsen J, Drobnik L, Sondore A. Anaesthesia workforce in Europe. European journal of anaesthesiology. 2007;24(12):991-1007.

5. Waters R. Pain relief for children. Am J Surg. 1938;39:470-475.

6. Smith C, Rowe RD, Vlad P. Sedation of children for cardiac catheterization with an ataractic mixture. Canadian Anaesthetists' Society journal. 1958;5(1):35-40.

7. Cote CJ, Karl HW, Notterman DA, Weinberg JA, McCloskey C. Adverse sedation events in pediatrics: analysis of medications used for sedation. Pediatrics. 2000;106(4):633-644.

8. Cote CJ, Notterman DA, Karl HW, Weinberg JA, McCloskey C. Adverse sedation events in pediatrics: a critical incident analysis of contributing factors. Pediatrics. 2000;105(4 Pt 1):805-814.

9. Guidelines for the elective use of conscious sedation, deep sedation, and general anesthesia in pediatric patients. Committee on Drugs. Section on anesthesiology. Pediatrics. 1985;76(2):317-321.

10. American Academy of Pediatrics Committee on Drugs: Guidelines for monitoring and management of pediatric patients during and after sedation for diagnostic and therapeutic procedures. Pediatrics. 1992;89(6 Pt 1):1110-1115.

11. Guidelines for monitoring and management of pediatric patients during and after sedation for diagnostic and therapeutic procedures: addendum. Pediatrics. 2002;110(4):836-838.

12. Cote $\mathrm{CJ}$, Wilson S. Guidelines for monitoring and management of pediatric patients during and after sedation for diagnostic and therapeutic procedures: an update. Pediatrics. 2006;118(6):2587-2602.

13. Practice guidelines for sedation and analgesia by non-anesthesiologists. Anesthesiology. 2002;96(4): 1004-1017.

14. SIGN Guideline 58: Safe sedation of children undergoing diagnostic and therapeutic procedures http://www.sign.ac.uk/pdf/sign58.pdf. Published 2004.

15. Guideline statement: management of procedure-related pain in children and adolescents. $J$ Paediatr Child Health. 2006;42 Suppl 1:S1-29.

16. Mace SE, Brown LA, Francis L, Godwin SA, Hahn SA, Howard PK, et al. Clinical policy: Critical issues in the sedation of pediatric patients in the emergency department. Annals of emergency medicine. 2008;51(4):378-399, 399 e371-357.

17. Pitetti RD, Singh S, Pierce MC. Safe and efficacious use of procedural sedation and analgesia by nonanesthesiologists in a pediatric emergency department. Archives of pediatrics \& adolescent medicine. 2003;157(11):1090-1096.

18. Sury M, Bullock I, Rabar S, Demott K. Sedation for diagnostic and therapeutic procedures in children and young people: summary of NICE guidance. BMJ (Clinical research ed.341:c6819.

19. Cote $\mathrm{CJ}$. Round and round we go: sedation -- what is it, who does it, and have we made things safer for children? Paediatric anaesthesia. 2008;18(1):3-8.

20. Folkes K. Is restraint a form of abuse? Paediatric nursing. 2005;17(6):41-44.

21. Cravero JP, Blike GT. Review of pediatric sedation. Anesthesia and analgesia. 2004;99(5):1355-1364.

22. Bhatt M, Kennedy RM, Osmond MH, Krauss B, McAllister JD, Ansermino JM, et al. Consensus-based recommendations for standardizing terminology and reporting adverse events for emergency department procedural sedation and analgesia in children. Annals of emergency medicine. 2009;53(4): 426-435 e424.

23. Barbi E, Gerarduzzi T, Marchetti F, Neri E, Verucci E, Bruno I, et al. Deep sedation with propofol by nonanesthesiologists: a prospective pediatric experience. Archives of pediatrics \& adolescent medicine. 2003;157(11):1097-1103. 
24. Hoffman GM, Nowakowski R, Troshynski TJ, Berens RJ, Weisman SJ. Risk reduction in pediatric procedural sedation by application of an American Academy of Pediatrics/American Society of Anesthesiologists process model. Pediatrics. 2002;109(2):236-243.

25. Pitetti R, Davis PJ, Redlinger R, White J, Wiener E, Calhoun KH. Effect on hospital-wide sedation practices after implementation of the 2001 JCAHO procedural sedation and analgesia guidelines. Archives of pediatrics \& adolescent medicine. 2006;160(2):211-216.

26. Polaner DM, Houck CS, Rockoff MA, Mancuso TJ, Finley GA, Maxwell LG, et al. Sedation, risk, and safety: do we really have data at last? Pediatrics. 2001;108(4):1006-1008.

27. Babl FE, Munro J, Kainey G, Palmer GM, Iser A. Scope for improvement: hospital wide sedation practice at a children's hospital. Archives of disease in childhood. 2006;91(8):716-717.

28. Holroyd I. Conscious sedation in pediatric dentistry. A short review of the current UK guidelines and the technique of inhalational sedation with nitrous oxide. Paediatric anaesthesia. 2008;18(1):13-17.

29. Babl FE, Oakley E, Seaman C, Barnett P, Sharwood LN. High-concentration nitrous oxide for procedural sedation in children: adverse events and depth of sedation. Pediatrics. 2008;121(3):e528-532.

30. Sury MR, Smith JH. Deep sedation and minimal anesthesia. Paediatric anaesthesia. 2008;18(1):18-24.

31. Vangerven M, Van Hemelrijck J, Wouters P, Vandermeersch E, Van Aken H. Light anaesthesia with propofol for paediatric MRI. Anaesthesia. 1992;47(8):706-707.

32. Green SM, Krauss B. The semantics of ketamine. Annals of emergency medicine. 2000;36(5):480-482.

33. Motas D, McDermott NB, VanSickle T, Friesen RH. Depth of consciousness and deep sedation attained in children as administered by nonanaesthesiologists in a children's hospital. Paediatric anaesthesia. 2004;14(3):256-260.

34. Alexander J, Manno M. Underuse of analgesia in very young pediatric patients with isolated painful injuries. Annals of emergency medicine. 2003;41(5):617-622.

35. MacLean S, Obispo J, Young KD. The gap between pediatric emergency department procedural pain management treatments available and actual practice. Pediatric emergency care. 2007;23(2):87-93.

36. Loryman B, Davies F, Chavada G, Coats T. Consigning "brutacaine" to history: a survey of pharmacological techniques to facilitate painful procedures in children in emergency departments in the UK. Emerg Med J. 2006;23(11):838-840.

37. Bhargava R, Young KD. Procedural pain management patterns in academic pediatric emergency departments. Acad Emerg Med. 2007;14(5):479-482.

38. Hain RD, Campbell C. Invasive procedures carried out in conscious children: contrast between North American and European paediatric oncology centres. Archives of disease in childhood. 2001;85(1):12-15.

39. Rennick JE, Johnston CC, Dougherty G, Platt R, Ritchie JA. Children's psychological responses after critical illness and exposure to invasive technology. J Dev Behav Pediatr. 2002;23(3):133-144.

40. Green SM, Krauss B. Barriers to propofol use in emergency medicine. Annals of emergency medicine. 2008;52(4):392-398.

41. Hardy M, Armitage G. The child's right to consent to $x$-ray and imaging investigations: issues of restraint and immobilization from a multidisciplinary perspective. J Child Health Care. 2002;6(2):107-119.

42. Cravero JP, Blike GT. Pride, Prejudice, and Pediatric Sedation:A Multispecialty Evaluation of the State of the Art. In: Report from a Dartmouth Summit on Pediatric Sedation: National Patient Safety Foundation; 2001.

43. Schechter NL. From the Ouchless Place to Comfort Central: the evolution of a concept. Pediatrics. 2008;122 Suppl 3:S154-160.

44. Malviya S, Voepel-Lewis T, Eldevik OP, Rockwell DT, Wong JH, Tait AR. Sedation and general anaesthesia in children undergoing MRI and CT: adverse events and outcomes. British journal of anaesthesia. 2000;84(6):743-748.

45. EACH charter 2001 (http://www.each-for-sick-children.org/each-charter). http://www.each-for-sickchildren.org/each-charter.

46. Malviya S, Voepel-Lewis T, Tait AR. Adverse events and risk factors associated with the sedation of children by nonanesthesiologists. Anesthesia and analgesia. 1997;85(6):1207-1213. 
47. Agrawal D, Manzi SF, Gupta R, Krauss B. Preprocedural fasting state and adverse events in children undergoing procedural sedation and analgesia in a pediatric emergency department. Annals of emergency medicine. 2003;42(5):636-646.

48. Babl FE, Puspitadewi A, Barnett P, Oakley E, Spicer M. Preprocedural fasting state and adverse events in children receiving nitrous oxide for procedural sedation and analgesia. Pediatric emergency care. 2005;21(11):736-743.

49. Bell A, Treston G, McNabb C, Monypenny K, Cardwell R. Profiling adverse respiratory events and vomiting when using propofol for emergency department procedural sedation. Emerg Med Australas. 2007;19(5):405-410.

50. Keidan I, Gozal D, Minuskin T, Weinberg M, Barkaly H, Augarten A. The effect of fasting practice on sedation with chloral hydrate. Pediatric emergency care. 2004;20(12):805-807.

51. Roback MG, Bajaj L, Wathen JE, Bothner J. Preprocedural fasting and adverse events in procedural sedation and analgesia in a pediatric emergency department: are they related? Annals of emergency medicine. 2004;44(5):454-459.

52. Treston G. Prolonged pre-procedure fasting time is unnecessary when using titrated intravenous ketamine for paediatric procedural sedation. Emerg Med Australas. 2004;16(2):145-150.

53. Ghaffar S, Haverland C, Ramaciotti C, Scott WA, Lemler MS. Sedation for pediatric echocardiography: evaluation of preprocedure fasting guidelines. J Am Soc Echocardiogr. 2002;15(9):980-983.

54. Green SM, Roback MG, Miner JR, Burton JH, Krauss B. Fasting and emergency department procedural sedation and analgesia: a consensus-based clinical practice advisory. Annals of emergency medicine. 2007;49(4):454-461.

55. Cravero JP, Blike GT, Beach M, Gallagher SM, Hertzog JH, Havidich JE, et al. Incidence and nature of adverse events during pediatric sedation/anesthesia for procedures outside the operating room: report from the Pediatric Sedation Research Consortium. Pediatrics. 2006;118(3):1087-1096.

56. Maxwell LG, Tobias JD, Cravero JP, Malviya S. Adverse effects of sedatives in children. Expert opinion on drug safety. 2003;2(2):167-194.

57. Green SM, Krauss B. Propofol in emergency medicine: pushing the sedation frontier. Annals of emergency medicine. 2003;42(6):792-797.

58. Hart LS, Berns SD, Houck CS, Boenning DA. The value of end-tidal CO2 monitoring when comparing three methods of conscious sedation for children undergoing painful procedures in the emergency department. Pediatric emergency care. 1997;13(3):189-193.

59. Lightdale JR, Goldmann DA, Feldman HA, Newburg AR, DiNardo JA, Fox VL. Microstream capnography improves patient monitoring during moderate sedation: a randomized, controlled trial. Pediatrics. 2006;117(6):e1170-1178.

60. McQuillen KK, Steele DW. Capnography during sedation/analgesia in the pediatric emergency department. Pediatric emergency care. 2000;16(6):401-404.

61. Miner JR, Heegaard W, Plummer D. End-tidal carbon dioxide monitoring during procedural sedation. Acad Emerg Med. 2002;9(4):275-280.

62. Tobias JD. End-tidal carbon dioxide monitoring during sedation with a combination of midazolam and ketamine for children undergoing painful, invasive procedures. Pediatric emergency care. 1999;15(3):173-175.

63. Tobias JD, Kavanaugh-McHugh A. Oximetry and capnography during sedation for transesophageal echocardiography. Clinical pediatrics. 1995;34(10):565-566.

64. Yldzdas D, Yapcoglu H, Ylmaz HL. The value of capnography during sedation or sedation/analgesia in pediatric minor procedures. Pediatric emergency care. 2004;20(3):162-165.

65. Krauss B, Hess DR. Capnography for procedural sedation and analgesia in the emergency department. Annals of emergency medicine. 2007;50(2):172-181.

66. Chernik DA, Gillings D, Laine H, Hendler J, Silver JM, Davidson AB, et al. Validity and reliability of the Observer's Assessment of Alertness/Sedation Scale: study with intravenous midazolam. Journal of clinical psychopharmacology. 1990;10(4):244-251. 
67. Malviya S, Voepel-Lewis T, Tait AR. A comparison of observational and objective measures to differentiate depth of sedation in children from birth to 18 years of age. Anesthesia and analgesia. 2006;102(2):389-394.

68. Malviya S, Voepel-Lewis T, Tait AR, Merkel S, Tremper K, Naughton N. Depth of sedation in children undergoing computed tomography: validity and reliability of the University of Michigan Sedation Scale (UMSS). British journal of anaesthesia. 2002;88(2):241-245.

69. Malviya S, Voepel-Lewis T, Ludomirsky A, Marshall J, Tait AR. Can we improve the assessment of discharge readiness?: A comparative study of observational and objective measures of depth of sedation in children. Anesthesiology. 2004;100(2):218-224.

70. Berkenbosch JW, Fichter CR, Tobias JD. The correlation of the bispectral index monitor with clinical sedation scores during mechanical ventilation in the pediatric intensive care unit. Anesthesia and analgesia. 2002;94(3):506-511; table of contents.

71. Grindstaff RJ, Tobias JD. Applications of bispectral index monitoring in the pediatric intensive care unit. Journal of intensive care medicine. 2004;19(2):111-116.

72. Tobias JD, Grindstaff R. Bispectral index monitoring during the administration of neuromuscular blocking agents in the pediatric intensive care unit patient. Journal of intensive care medicine. 2005;20(4):233237.

73. Powers KS, Nazarian EB, Tapyrik SA, Kohli SM, Yin H, van der Jagt EW, et al. Bispectral index as a guide for titration of propofol during procedural sedation among children. Pediatrics. 2005;115(6):1666-1674.

74. Malviya S, Voepel-Lewis T, Tait AR, Watcha MF, Sadhasivam S, Friesen RH. Effect of age and sedative agent on the accuracy of bispectral index in detecting depth of sedation in children. Pediatrics. 2007;120(3):e461-470.

75. Deitch K, Chudnofsky CR, Dominici P. The utility of supplemental oxygen during emergency department procedural sedation and analgesia with midazolam and fentanyl: a randomized, controlled trial. Annals of emergency medicine. 2007;49(1):1-8.

76. Deitch K, Chudnofsky CR, Dominici P. The utility of supplemental oxygen during emergency department procedural sedation with propofol: a randomized, controlled trial. Annals of emergency medicine. 2008;52(1):1-8.

77. Green SM, Krauss B. Supplemental oxygen during propofol sedation: yes or no? Annals of emergency medicine. 2008;52(1):9-10.

78. Patel R, Lenczyk M, Hannallah RS, McGill WA. Age and the onset of desaturation in apnoeic children. Canadian journal of anaesthesia = Journal canadien d'anesthesie. 1994;41(9):771-774.

79. Xue FS, Luo LK, Tong SY, Liao X, Deng XM, An G. Study of the safe threshold of apneic period in children during anesthesia induction. Journal of clinical anesthesia. 1996;8(7):568-574.

80. Newman DH, Azer MM, Pitetti RD, Singh S. When is a patient safe for discharge after procedural sedation? The timing of adverse effect events in 1367 pediatric procedural sedations. Annals of emergency medicine. 2003;42(5):627-635.

81. Steurer LM, Luhmann J. Adverse effects of pediatric emergency sedation after discharge. Pediatric nursing. 2007;33(5):403-407, 426; quiz 409.

82. Green SM, Johnson NE. Ketamine sedation for pediatric procedures: Part 2, Review and implications. Annals of emergency medicine. 1990;19(9):1033-1046.

83. Hertzog JH, Campbell JK, Dalton HJ, Hauser GJ. Propofol anesthesia for invasive procedures in ambulatory and hospitalized children: experience in the pediatric intensive care unit. Pediatrics. 1999;103(3):E30.

84. Hertzog JH, Dalton HJ, Anderson BD, Shad AT, Gootenberg JE, Hauser GJ. Prospective evaluation of propofol anesthesia in the pediatric intensive care unit for elective oncology procedures in ambulatory and hospitalized children. Pediatrics. 2000;106(4):742-747.

85. Cravero JP, Beach ML, Blike GT, Gallagher SM, Hertzog JH. The incidence and nature of adverse events during pediatric sedation/anesthesia with propofol for procedures outside the operating room: a report from the Pediatric Sedation Research Consortium. Anesthesia and analgesia. 2009;108(3):795-804.

86. Couloures KG, Beach M, Cravero JP, Monroe KK, Hertzog JH. Impact of provider specialty on pediatric procedural sedation complication rates. Pediatrics. 2011;127(5):e1154-1160. 
CHAPTER 2

PSA in Dutch general paediatrics

An analysis of safety and effectiveness 



\section{Part 2.1 Adherence to safety guidelines on paediatric procedural sedation and Analgesia (PSA): the results of a nationwide survey under general paediatricians in The Netherlands}




\section{Abstract}

Objectives: Following two fatal accidents during paediatric procedural sedation and analgesia (PSA) in The Netherlands, we investigated the level of adherence to established safety standards on PSA in a nationwide cohort of fully trained general paediatricians, entrusted with PSA.

Study Design and methods: Sample survey. Safety guidelines on PSA were split into 4 domains ('Pre-sedation Assessment', 'Monitoring during PSA', 'Recovery after PSA' and 'Facilities and Competences for Emergencies and Rescue'). Each domain was operationalized into subdomains and items. Items were presented within a questionnaire list as procedural points of attention on which respondents could give their personal adherence score. Percentages of full adherence were calculated. Non-adherence was defined as gradual deviation from full adherence. After factor and reliability analysis, observed scores were summed up to scales and results were transformed to a $0-10$ report mark (RM). A RM of $\geq 9$ is considered as a satisfactory level of adherence while an $\mathrm{RM}<6$ is considered as unacceptably low.

Results: Full adherence was rare. For most (sub)domains only a minority of respondents achieved a satisfactory level of adherence. Large numbers of respondents had scores below 6 .

Conclusions: Potentially unsafe PSA practices are common under Dutch general paediatricians, despite the availability of guidelines. The design of guidelines should include a goal-directed plan for implementation including training, initiatives for continuous quality assurance and improvement and repeated measurements of adherence to guidelines. 


\section{Introduction}

\section{What is already known about this subject?}

Following sedation related accidents in children undergoing dental procedures, the American Academy of Paediatrics (AAP) published a first guideline on procedural sedation and Analgesia (PSA) in 1985. Later on it was shown that significant contributing factors to PSA related accidents were out of hospital settings, inadequate presedation assessment, inappropriate monitoring, inadequate recovery procedures and inadequate resuscitation skills. As a result specific guidelines on paediatric PSA were published worldwide. All these guidelines specify similar safety precautions that include (1) assessment of the sedation risk prior to PSA, (2) informed consent, (3) guidelines on proper fasting status, (4) appropriate monitoring, (5) recovery standards (6) professional competence, and (7) imperative rescue facilities. In small test populations it has been shown that adherence to these guidelines reduces the occurrence of adverse events.

However, it is unknown to what level professionals adhere to new guidelines on PSA following their publication.

\section{What does this study add?}

1. This is the first study analyzing nationwide adherence to guidelines on paediatric procedural sedation. Since a prospective, observational analysis of the 'real PSA world' is hardly achievable in this respect, we chose an indirect, retrospective method based on self-report of common practice. The study shows that, despite the availability of specific guidelines (that even were adopted by the national society of paediatrics as the official guidelines on this topic), potentially unsafe sedation practices are widespread in a population of general paediatricians entrusted with PSA. Designing guidelines is of no benefit, if a specific strategy for implementation is neglected.

2. Non-adherence was considered in this study as gradual deviation from full adherence. Therefore an original methodology had to be designed. This method may be applicable in future similar research.

Due to limited anaesthesia services, Procedural Sedation and Analgesia (PSA) is often provided by non-anaesthesiologists. ${ }^{1}$ Concerns about safety have led to the publication of guidelines on PSA. ${ }^{2-8}$ In general, these guidelines include the assessment of sedation-related risks, proper fasting, appropriate monitoring, recovery standards, professional competence, and rescue facilities. Guideline implementation leads to safer PSA. ${ }^{9-11}$ However, local persistence of unsafe practices have been reported. ${ }^{12}$ In the Netherlands PSA for common procedures (e.g. diagnostic imaging) in hospitalized children is mostly prescribed by general paediatricians. In 1998 the Dutch Society of Paediatrics adopted a national guideline on PSA. ${ }^{13}$ This guideline, as well as more recent international guidelines, has been addressed by several national symposia and publications. Nevertheless, since 1998 two fatal accidents happened in children during PSA for diagnostic imaging. Existing safety guidelines had not been implemented in these cases. The question rose whether these incidents were isolated events or indications for widespread nonadherence. Therefore we investigated the adherence to PSA guidelines in the Dutch general paediatric practice. 


\section{Methods}

A postal questionnaire was sent to all general paediatricians working in hospitals in the Netherlands in January 2007, as registered by the Dutch Society of Paediatrics. Definitions of PSA were provided in an introduction. Respondents were requested to focus on procedures requiring moderate or deep sedation. Besides questions on individual background characteristics, the questionnaire included questions on adherence to established safety standards on PSA. Standards were selected from international guidelines on PSA, published between 1998 and 2006. ${ }^{2-8}$ A multidisciplinary panel of the Dutch Institute for Health Care Improvement, involved in the design of a new evidence-based guideline on PSA, performed the selection process. Standards selected were divided into 4 domains ('Presedation Assessment', 'Monitoring during PSA', 'Recovery after PSA' and 'Facilities and Competences for Emergencies and Rescue') and 16 subdomains. Each subdomain was operationalized into items, which successively stress a specific safety aspect during PSA. (Table 1) Items were presented within a questionnaire list as procedural points of attention on which respondents could give their personal adherence score: 'never' done ('0'), 'seldom' done (' 1 '), 'often' done ('2') or 'always' done (' 3 '). Respondents could also indicate that they 'do not know', if the item was done at all.

Observed 4-point Likert-scores were dichotomized into a new score '0' ('no full adherence', observed scores ' 0 ', ' 1 ' or ' 2 ') and a new score ' 1 ' ('full adherence', observed score ' 3 '). Univariate statistics then consist of percentages of full adherence. Missing scores on items ('don't know' or 'no answer') were counted as non-adherence.

Table 1: Adherence to established safety standards for PSA: percentages of full adherence and statistical characteristic domains and subdomains in this study

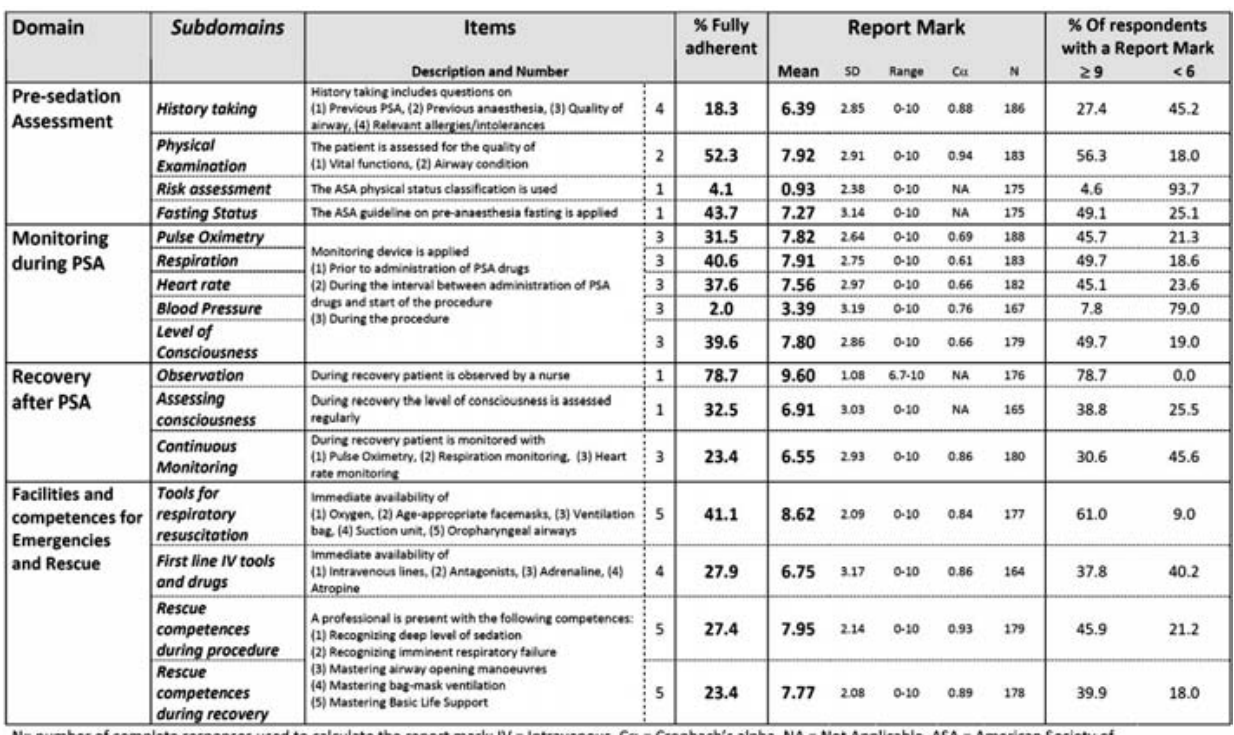

$N=$ number of complete responses used to calculate the report mark; IV = Intravenous, $C \alpha=C$ Cronbach's alpha, NA = Not Applicable, ASA = American Society of Anaesthesiologists, PSA=Procedural Sedation and Analgesia.

Alternatively, non-adherence can be considered as a gradual deviation from perfect adherence. Assuming equal distances in the gradual scoring, means and standard deviations of items and overall scales were calculated. Pearson product-moment correlation coefficients were used to 
measure the (linear) relationships between multiple subdomain items. Principal component analysis (PCA) with oblique rotation was performed to examine the internal factorial cohesion in and between items. Listwise deletion of missing cases was used. Factor-loadings on intended subdomains had to be $>0.50$. Cronbach's alpha was calculated for each factor found in PCA to measure its level of internal consistency. Missing values on items were considered as invalid and kept out of overall statistics. If sufficient numbers of items had valid 0-3 scores, scale construction was done by summing these scores and dividing the result by the number of validly scored items. Resulting scale scores and single item subdomain scores were mathematically transformed to a 0-10 report mark (RM) score, where ' 0 ' stands for absolute non-adherence and ' 10 ' for full adherence. A RM of $\geq 9$ is considered as an excellent level of adherence while an RM $<6$ is considered as unacceptably low. All statistics were analyzed with SPSS-pc version 15.0.

\section{Results}

\section{Response rate and respondent's characteristics}

In 2007428 specialists in The Netherlands had general paediatrics as their main clinical activity. The questionnaire was returned by 258 of them (60.2\%). 197 paediatricians (76.4\%) reported to have performed PSA personally during the preceding 12 months. Their responses were sufficiently complete for further data processing and analysis. Respondents represented general paediatricians working in 88 of the 97 Dutch hospitals (90.7\%). Demographics and regional distribution of respondents were similar to those of the total population of Dutch general paediatricians (data not shown). Procedures for which respondents reported to use moderate or deep sedation were diagnostic imaging (81.7\%), wound care (33.5\%), endoscopy (27.9\%) and lumbar puncture (27.9\%). Drugs of choice were chloral hydrate $(81.2 \%)$, midazolam (72.6\%), morphine (37.6\%), combinations of midazolam with morphine or chloral hydrate (78.3\%), (lytic) cocktails (20.8\%) and ketamine (10.1\%).

\section{Adherence to guidelines}

Full adherence occurred in $<25 \%$ of respondents for the subdomains history taking, risk assessment, blood pressure monitoring during PSA, monitoring during recovery and rescue competence present during recovery. (Table 1) For most subdomains less than $50 \%$ of respondents achieved a satisfactory level of self-professed adherence ( $R M \geq 9$ ). More than $25 \%$ of respondents scored RM's $<6$ for the subdomains history taking, risk assessment, fasting status, blood pressure monitoring during PSA, assessing consciousness during recovery, monitoring during recovery and the immediate availability of intravenous tools and drugs for resuscitation.

\section{Discussion}

Despite the availability of guidelines, we found that full adherence to established safety standards on PSA is strikingly rare among Dutch general paediatricians. However, it is unlikely that the naïve definition of full adherence (i.e. all items are always and simultaneously applied) is a 
just or even realistic one. A high level of adherence (i.e. nearly all items are applied nearly always) is probably sufficient to obtain optimal safety. Therefore we considered it more valid to define non-adherence as a 'gradual deviation from full adherence', rather than as 'absence of full adherence'. Gradual deviation from perfect behaviour can be expressed by report marks (RM). RM's, as ordinal scales of performance, may elucidate which particular subdomains are in need for improvement and to what extent improvement is indicated. They may also - in repeated surveys - quantify individual or collective progression or deterioration, and - in more experimental settings - measure the results of care improvement project.

In this study only a minority of the respondents achieved a high level of self-professed adherence, defined as a RM of $\geq 9$. As for now it is unknown at which cut-off level an acceptable safety level is guaranteed. However, we speculate that a $R M \geq 9$ is likely to be associated with a high level of safety because this mark implies a nearly systematic application of all contributing items. This reasoning is in line with the observed reduction in adverse events during anaesthesia by the adoption of a uniform safety-directed practice. ${ }^{14}$ Conversely, a RM $<6$ means that most items are adopted only occasionally or even not applied at all. We believe that below this level a patient will be systematically at risk for unexpected and unpleasant events within the scope of a particular subdomain. The assumed relationship between RM's and safety level must be validated in future studies.

There are several explanations for the observed low levels of adherence in Dutch general paediatricians. Firstly, training in PSA is not formally incorporated during residency and a particular final attainment level is non-existing. Secondly, PSA is not considered as a distinct medical competence or act. This makes that PSA finds itself in a twilight zone, lacking formal registration and quality assurance. Next, following the publication of the national guideline a program for implementation was neglected. Other explanations are the fact that indications, contraindications and risks associated with PSA are not widely known and not widely recognized. More over financial restrictions may apply in the sense that there is a lack of equipment, personnel and facilities to practice formal PSA. Moreover the procedure is not reimbursed in many cases.

As any self-report survey, data may be subject to social desirability bias, possibly leading to an underestimation of non-adherence. Since PSA may also be entrusted to trainees, this study in fully trained paediatricians might not reflect the whole PSA reality. The retrospective character of self-report is probably another disadvantage. Respondents had to average their adherence behaviour over the past 12 months from memory. Furthermore, adherence cannot be operationalized and quantified in an interval or ratio scale, so report mark results are basically of an ordinal nature. Practical application of parametric statistics must therefore be regarded with caution.

In conclusion we demonstrated that, despite the availability of specific guidelines, adherence to established safety standards on PSA is low in Dutch general paediatrics. The two fatal incidents are likely to be only the top of an iceberg of widespread unsafe PSA practices. Simply publishing guidelines does not automatically imply change of practice. ${ }^{15}$ Therefore, guidelines must be accompanied by a strategy for implementation. At first, a minimal level of competence must be defined for PSA in children. Next, compulsive training programs must be designed for professionals entrusted with PSA. Existing training documents published by the Royal College of Anaesthesiology could be used for this purpose. Furthermore, implementation should include formalizing PSA as a distinctive medical act, initiatives for Quality Assurance and Improvement (QA/QI), rigorous registration of practices and adverse events, and repeated measurements of adherence. We feel that only well-trained and competent professionals can achieve and main- 
tain a high level of adherence to established safety guidelines. Only such professionals should be credentialed to perform PSA.

\section{Acknowledgements}

The authors like to thank Sera Schniedermeier, MSc, for her meticulous data processing 


\section{References}

1. Cote CJ. Discharge criteria for children sedated by nonanesthesiologists: is "safe" really safe enough? Anesthesiology. 2004;100(2):207-209.

2. Guidelines for monitoring and management of pediatric patients during and after sedation for diagnostic and therapeutic procedures: addendum. Pediatrics. 2002;110(4):836-838.

3. Practice guidelines for sedation and analgesia by non-anesthesiologists. Anesthesiology. 2002;96(4): 1004-1017.

4. Yldzdas D, Yapcoglu H, Ylmaz HL. The value of capnography during sedation or sedation/analgesia in pediatric minor procedures. Pediatric emergency care. 2004;20(3):162-165.

5. Guideline statement: management of procedure-related pain in children and adolescents. $J$ Paediatr Child Health. 2006;42 Suppl 1:S1-29.

6. Cote $\mathrm{CJ}$, Wilson S. Guidelines for monitoring and management of pediatric patients during and after sedation for diagnostic and therapeutic procedures: an update. Pediatrics. 2006;118(6):2587-2602.

7. Krauss B, Green SM. Procedural sedation and analgesia in children. Lancet. 2006;367(9512):766-780.

8. SIGN Guideline 58: Safe sedation of children undergoing diagnostic and therapeutic procedures http://www.sign.ac.uk/pdf/sign58.pdf. Published 2004.

9. Barbi E, Gerarduzzi T, Marchetti F, Neri E, Verucci E, Bruno I, et al. Deep sedation with propofol by nonanesthesiologists: a prospective pediatric experience. Archives of pediatrics \& adolescent medicine. 2003;157(11):1097-1103.

10. Hoffman GM, Nowakowski R, Troshynski TJ, Berens RJ, Weisman SJ. Risk reduction in pediatric procedural sedation by application of an American Academy of Pediatrics/American Society of Anesthesiologists process model. Pediatrics. 2002;109(2):236-243.

11. Pitetti R, Davis PJ, Redlinger R, White J, Wiener E, Calhoun KH. Effect on hospital-wide sedation practices after implementation of the 2001 JCAHO procedural sedation and analgesia guidelines. Archives of pediatrics \& adolescent medicine. 2006;160(2):211-216.

12. Babl FE, Munro J, Kainey G, Palmer GM, Iser A. Scope for improvement: hospital wide sedation practice at a children's hospital. Archives of disease in childhood. 2006;91(8):716-717.

13. Knape J. Sedation and/or analgesia by non-anesthesiologists [Sedatie en/of Analgesie door nietanesthesiologen]. Consensu Guideline 1998 www.cbo.nl. www.cbo.nl.

14. Polaner DM, Houck CS, Rockoff MA, Mancuso TJ, Finley GA, Maxwell LG, et al. Sedation, risk, and safety: do we really have data at last? Pediatrics. 2001;108(4):1006-1008.

15. Sheldon TA, Cullum N, Dawson D, Lankshear A, Lowson K, Watt I, et al. What's the evidence that NICE guidance has been implemented? Results from a national evaluation using time series analysis, audit of patients' notes, and interviews. BMJ (Clinical research ed. 2004;329(7473):999. 


\section{Part 2.2 Safe and effective procedural sedation for}

magnetic resonance imaging in children: daily practice in The Netherlands compared with the results of a systematic literature review. 


\begin{abstract}
Objectives: During magnetic resonance imaging (MRI) scans, young children and children with cognitive impairment need some form of procedural sedation (PS) in order to lie motionless for a sufficient length of time. Some serious and even fatal incidents have occurred with uncontrolled PS for MRI over the last decade in the Netherlands. It has been demonstrated that essential safety guidelines in relation to PS have been insufficiently implemented in general paediatric practice in the Netherlands. In addition, there are some concerns about the effectiveness of frequently used PS procedures.
\end{abstract}

Study design and methods: Using a survey, we examined which PS techniques and medicines are mostly used by general paediatricians for MRI in children. Normal practice was compared with the results of a systematic review (SR) of recent literature. By means of the SR, we have tried to answer the clinical question as to which is the safest and most effective technique for PS during MRI in childhood.

Results and condlusions: Dutch paediatricians rarely use anaesthesia for MRI. They mostly use PS based on chloral hydrate, midazolam and/or lytic cocktails. These sedatives have less than optimal effectiveness. It is not known how many examinations fail partially or completely as a result and failure of such a procedure is also very costly. Yet, these sedative drugs are associated with a significant risk of complications. Their use must therefore always be associated with extensive precautionary measures and the direct availability or preferably presence of competent professionals. Propofol has the highest level of effectiveness of all sedatives. Propofol can be used safely by competent non-anaesthesiologists. If these professionals are not available, it is recommended that children who are in need for sedation to undergo MRI are referred to an anaesthesiologist. 


\section{Introduction}

Magnetic resonance imaging (MRI) is an advanced imaging technique, the quality of which is partly determined by immobility during the procedure. Since young children and children with mental retardation are often not able to lie still for a sufficient length of time, there is a need for procedural sedation. $^{1,2}$

Procedural sedation (PS) is defined as the administration of sedatives, sedating analgesia, or dissociative medicines in order to achieve analgesia, sedation, movement control, and reduced anxiety during a painful and/or unpleasant procedure. ${ }^{3}$ Due to the limited availability of anaesthesiological support, non-anaesthesiologists have developed their own PS protocols. Concerns have arisen during the last decade about the safety and effectiveness of this practice. ${ }^{4,5}$ Reports on serious incidents have led to safety guidelines across the world. ${ }^{3,6-9}$ Despite the existence of national guidelines (CBO-1998), serious incidents have also occurred during PS for MRI scans in the Netherlands. It has been demonstrated that essential safety guidelines regarding PS in the Netherlands have been insufficiently implemented in practice. ${ }^{10}$ The moderate effectiveness of many PS procedures is also causing concern. As a result of the low level of predictability of frequently used sedatives and the limited skills of non-anaesthesiologists, MRI scans regularly fail.

In this study, we have compiled an inventory of the methods and medicines used by general paediatricians in the Netherlands for PS during MRI scans. The findings were compared with the results of a systematic survey of literature. Some recommendations were drawn up on the basis of the literature review.

\section{Methodology}

\section{a. Measuring normal PS practice}

A questionnaire was sent to all paediatricians practicing general paediatric medicine in any one of the 97 Dutch hospitals for at least $50 \%$ of their working time in 2008 . The list of addressees was based on the members list of the Dutch Society of Paediatrics and the telephone numbers of all paediatric departments. The questionnaire contained questions concerning each paediatrician's PS practice for MRI examinations. A distinction was made between three age groups $<6$ months; 6-36 months; 3-8 years). For each age category, the respondent was given a choice of three options: anaesthesia, a non-pharmacological technique, or PS either on its own or in combination with a non-pharmacological technique. The last two options were then explored in greater detail.

\section{b. Systematic literature review}

An independent literature study was conducted by two authors (PL and JV). Searches in the English language were carried out using the search engines of Medline, Cochrane Library, and Embase for the Medical Subject Heading (MESH) search entries "Conscious sedation/all", "Moderate sedation/all", "Deep sedation/all", and the independent search terms "sedation", " $p(a)$ ediatric sedation", and "procedural sedation". The search was limited to the age group 018 years and to articles published between January 1995 and October 2010 in Dutch, English, French, and German. Studies for which only abstracts were available were not included. The first 
results were systematically combined with the MESH entry "Magnetic Resonance Imaging/all". Comparable combined searches were repeated for all medicines that were regularly used by Dutch general paediatricians for PS for the purpose of MRI. The search was repeated for all other medicines registered in the Netherlands that were reported in literature as being used for PS during MRI scans in children. Manuals and reference tables were examined for the existence of unknown studies. Articles were selected based on the extent to which they described the effectiveness and safety of the sedation achieved. An optimally effective PS technique was defined as a technique that (A) guarantees virtually $100 \%$ predictable procedural success as well as patient comfort, (B) enables optimal synchronization with the planned time of examination, and (C) is characterized by a minimal induction and recovery period, preventing any unnecessary strain on the care system. Only studies about PS during MRI scan on spontaneously breathing, nonintubated, and not seriously ill people (ASA I and II) were included. The results were processed in an evidence table, summarizing the data on effectiveness, complications, and late adverse effects. Articles were divided into categories based on the quality of the methodology used: $A 1$ : systematic review based on at least two independent A2 studies, A2: randomized, double-blind research, B1: comparative, prospective, non-randomized research, B2: retrospective comparative research, and C: non-comparative research.

\section{Results}

\section{a. Response}

At the beginning of 2008,428 specialists in The Netherlands were active for at least $50 \%$ of their working time on activities as a general paediatrician. Questionnaires were sent to all these 428 paediatricians. Of the 258 respondents, 182 (70,5\%) had performed a PS for an MRI examination during the previous year. On the assumption that this is a representative percentage, the study population was estimated to be 302 specialists $(0,705 \times 428)$. The relative sample can therefore be estimated at $60.3 \%(182 / 302)$. Respondents represented 88 of the $97(90,2 \%)$ paediatric departments.

\section{b. Most frequently applied PS techniques}

Only a small minority deployed anaesthesia. The majority applied some form of PS, sometimes in combination with a non-pharmacological technique. For children under six months old, a nonpharmacological technique was used most of the time. (Table 1a) The most used pharmaceutical products (alone or in combinations) were chloral hydrate (mostly rectally), midazolam (mostly intravenously), and lytic cocktails. Also regularly used were pethidine and antihistamines. (Table 1b) Swaddling (with young infants), a pre-procedural feed, having parents present, and playtherapeutical preparation or support were the most frequently applied non-pharmacological techniques. No distraction techniques or simulation were reported. Dutch paediatricians frequently combined sedation with swaddling or with a pre-procedural feed. (Table 1c) 
Table 1a: Overview of techniques reported by Dutch general paediatricians as their first choice for obtaining cooperation and immobility during MRI examinations of children (percentages).

\begin{tabular}{llll}
\hline & $<6$ months & $\mathbf{6 - 3 6}$ months & $\mathbf{3 - 8}$ years \\
\hline Anesthesia* & $34(19.4)$ & $43(25.4)$ & $27(15.0)$ \\
Non-pharmacological technique (NFT) & $53(30.3)$ & $6(3.6)$ & $29(16.1)$ \\
PS (Total) & $88(50.3)$ & $120(71.0)$ & $124(68.9)$ \\
PS + NFT & 88 & 86 & 100 \\
PS without NFT & 0 & 34 & 24 \\
Total** & $175(100)$ & $169(100)$ & $180(100)$ \\
\hline
\end{tabular}

* This means: a form of anesthesia or sedation performed by an anesthesiologist

** Since not every respondent had experience with every age category, the totals lie somewhat below 182.

Table 1b: Overview of medicines that Dutch general paediatricians reported using for PS during MRI examinations of children. Combinations of these medicines may have been used (percentages).

\begin{tabular}{llll}
\hline & $<6$ months & $6-36$ months & 3-8 years \\
\hline Chloral hydrate & $85(96.6)$ & $87(72.5)$ & $75(60.5)$ \\
Midazolam & $29(32.9)$ & $53(44.2)$ & $63(50.8)$ \\
Lytic cocktail & $11(12.5)$ & $36(30.0)$ & $32(25.8)$ \\
Pethidine & $1(1.1)$ & $9(7.5)$ & $9(7.3)$ \\
Morphine & $3(3.4)$ & $0(0.0)$ & $0(0.0)$ \\
Alimemazine & $4(4.5)$ & $5(4.2)$ & $6(4.8)$ \\
Promethazine & $0(0.0)$ & $3(2.5)$ & $5(4.0)$ \\
Pentobarbital & $1(1.1)$ & $1(0.8)$ & $0(0.0)$ \\
Thiopental & $1(1.1)$ & $2(1.7)$ & $0(0.0)$ \\
Diazepam & $0(0.0)$ & $3(2.5)$ & $3(2.4)$ \\
Clonazepam & $1(1.1)$ & $0(0.0)$ & $0(0.0)$ \\
Ketamine & $0(0.0)$ & $2(1.7)$ & $2(1.6)$ \\
Haloperidol & $0(0.0)$ & $1(0.8)$ & $0(0.0)$ \\
Trimipramine & $0(0.0)$ & $1(0.8)$ & $0(0.0)$ \\
Total & $88(100)$ & $120(100)$ & $124(100)$ \\
\hline
\end{tabular}

Table 1c: Overview of non-pharmacological techniques that Dutch general paediatricians reported using during MRI examinations of children. Combinations of these techniques may have been used (percentages).

\begin{tabular}{llll}
\hline & $<6$ months & $\mathbf{6 - 3 6 \text { months }}$ & $\mathbf{3 - 8}$ years \\
\hline Preprocedural feed & $128(90.8)$ & $46(50.0)$ & $9(6.9)$ \\
Swaddling or restraint & $59(41.8)$ & $20(21.7)$ & $7(5.4)$ \\
Presence of parent(s) & $80(62.5)$ & $84(91.3)$ & $124(96.1)$ \\
Distraction technique & $1(0.7)$ & $2(2.2)$ & $4(3.1)$ \\
Play therapy & $34(24.1)$ & $84(91.3)$ & $128(99.2)$ \\
& & & \\
Established combinations for PS & & $20(21.7)$ & $7(5.4)$ \\
Swaddling/restraint + PS & $36(25.5)$ & $42(45.6)$ & $9(6.9)$ \\
Preprocedural feed + PS & $75(53.2)$ & $92(100)$ & $129(100)$ \\
Total & $141(100)$ & & \\
\hline
\end{tabular}




\begin{tabular}{|c|c|c|c|c|c|c|c|}
\hline \multirow{4}{*}{\multicolumn{2}{|c|}{ 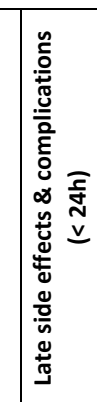 }} & 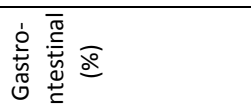 & $\frac{\infty}{z}$ & $\frac{\alpha}{z}$ & $\frac{\infty}{2}$ & $\frac{\infty}{2}$ & $\frac{\alpha}{2}$ \\
\hline & & 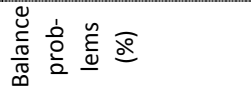 & $\frac{\alpha}{2}$ & $\frac{⿱}{z}$ & $\frac{\alpha}{2}$ & $\underset{\text { F }}{\mathcal{H}}$ & $\frac{\infty}{z}$ \\
\hline & & 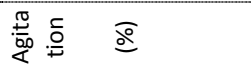 & $\frac{\infty}{z}$ & $\frac{\alpha}{2}$ & $\frac{\infty}{z}$ & $\stackrel{\text { n }}{\rightarrow}$ & $\frac{\infty}{z}$ \\
\hline & & 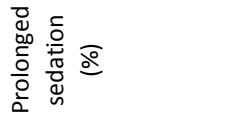 & $\frac{\infty}{z}$ & $\frac{\infty}{z}$ & $\frac{\infty}{2}$ & $\frac{⿱}{z}$ & $\frac{\alpha}{z}$ \\
\hline \multirow{6}{*}{\multicolumn{2}{|c|}{ 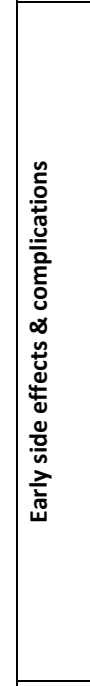 }} & 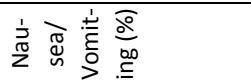 & $\frac{\infty}{z}$ & $\frac{\alpha}{z}$ & \multirow{6}{*}{ 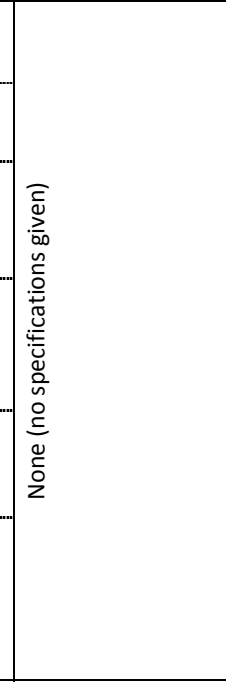 } & ฉ̃ & $\stackrel{i}{i}$ \\
\hline & & 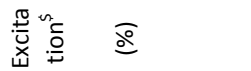 & $\frac{\infty}{z}$ & $\frac{\alpha}{z}$ & & $\frac{\infty}{2}$ & $\frac{\alpha}{z}$ \\
\hline & & 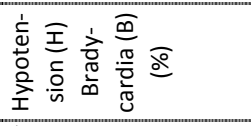 & 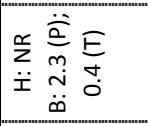 & $\frac{\kappa}{z}$ & & $\frac{\infty}{z}$ & $\frac{\infty}{z}$ \\
\hline & & 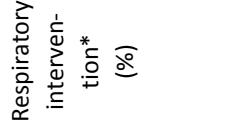 & \multirow{3}{*}{ 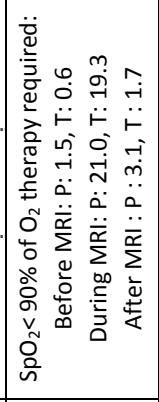 } & $\frac{\infty}{z}$ & & 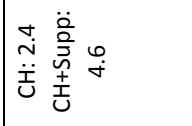 & $\stackrel{\circ}{\circ}$ \\
\hline & & 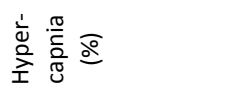 & & 产 & & $\frac{\infty}{z}$ & $\frac{⿱}{z}$ \\
\hline & & 离 & & $\frac{\propto}{2}$ & & 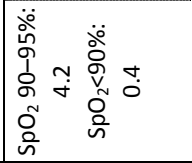 & $\stackrel{9}{i}$ \\
\hline & & 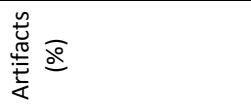 & $\frac{⿱}{z}$ & $\frac{\kappa}{z}$ & $\frac{⿱}{z}$ & $\frac{⿱}{z}$ & $\frac{\infty}{z}$ \\
\hline & $\ddot{ٍ ్ ~}$ & 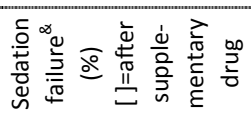 & $\begin{array}{ll}0 & m \\
0 & \stackrel{m}{+} \\
\dot{0} & \stackrel{\leftrightarrow}{*}\end{array}$ & 苟 & 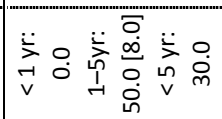 & 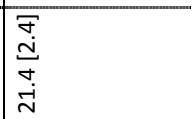 & 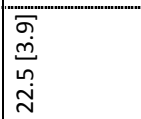 \\
\hline & 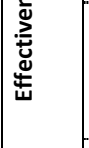 & 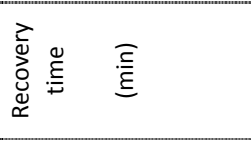 & 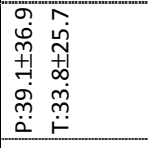 & $\frac{\infty}{z}$ & $\frac{\infty}{2}$ & 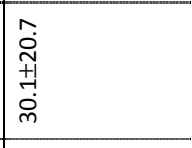 & $\begin{array}{l}9 \\
-1 \\
0 \\
+1 \\
-1 \\
6 \\
\end{array}$ \\
\hline & & 飬导 & $\frac{\alpha}{z}$ & $\frac{\alpha}{z}$ & $\frac{\infty}{z}$ & $\begin{array}{l}\hat{i} \\
\dot{i} \\
+1 \\
\dot{y} \\
\dot{y}\end{array}$ & 芦 \\
\hline \multicolumn{3}{|c|}{ 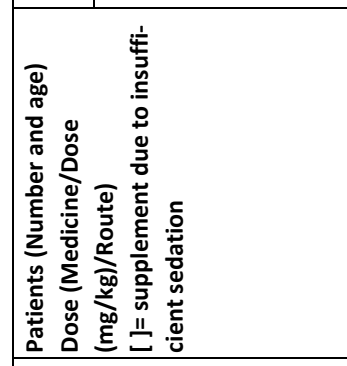 } & 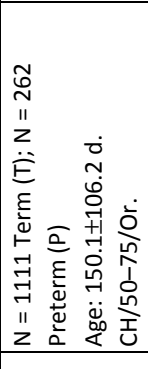 & 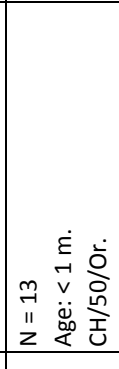 & 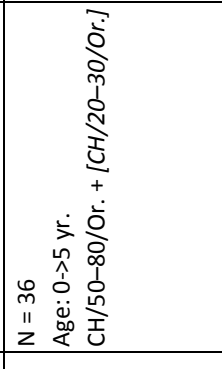 & 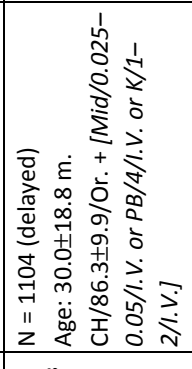 & 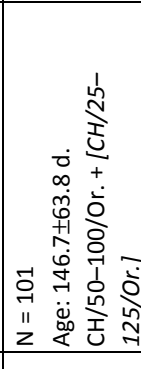 \\
\hline \multicolumn{3}{|c|}{ 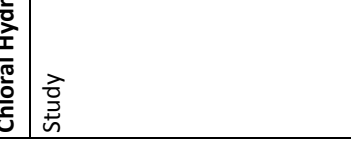 } & 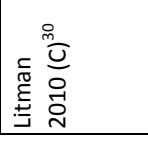 & 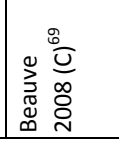 & 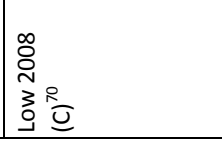 & 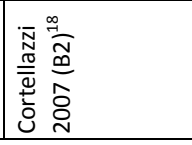 & 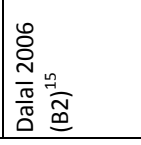 \\
\hline
\end{tabular}




\begin{tabular}{|c|c|c|c|c|c|c|}
\hline$\frac{\alpha}{z}$ & 孚 & m. & \multirow{4}{*}{ 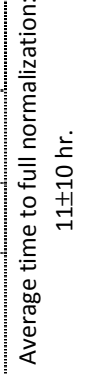 } & 魚 & \multirow{4}{*}{ 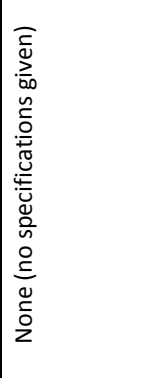 } & $\frac{\kappa}{z}$ \\
\hline$\frac{\alpha}{z}$ & $\frac{x}{z}$ & $\frac{\alpha}{z}$ & & $\frac{\alpha}{z}$ & & $\frac{\kappa}{z}$ \\
\hline$\frac{\alpha}{z}$ & 孚 & นn & & $\frac{\kappa}{z}$ & & $\frac{\alpha}{z}$ \\
\hline$\frac{⿱}{z}$ & $\frac{\alpha}{z}$ & $\stackrel{\circ}{\circ}$ & & $\frac{\alpha}{z}$ & & $\frac{\alpha}{z}$ \\
\hline 受 & 愛 & $\stackrel{m}{m}$ & $\exists$ & $\frac{\alpha}{z}$ & ma & $\frac{\alpha}{z}$ \\
\hline$\frac{⿱}{z}$ & $\frac{\alpha}{z}$ & $\because$ & $\because$ & 魚 & $\stackrel{m}{o}$ & $\frac{\alpha}{z}$ \\
\hline$\frac{\alpha}{z}$ & $\mid \begin{array}{ll}0 & 0 \\
0 & 0 \\
\ddot{I} & \ddot{\varphi}\end{array}$ & $\frac{\mathscr{c}}{z}$ & $\frac{\alpha}{z}$ & 魚 & $\frac{\mathscr{c}}{z}$ & $\frac{\mathscr{c}}{z}$ \\
\hline$\frac{⿱}{z}$ & ڤ̃ & 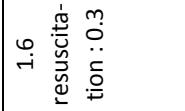 & $\stackrel{0}{0}$ & 鱼 & $\overrightarrow{7}$ & $\frac{⿱}{z}$ \\
\hline$\frac{⿱ ㇒}{z}$ & 受 & 受 & 愛 & 魚 & 愛 & 愛 \\
\hline$\frac{\kappa}{z}$ & 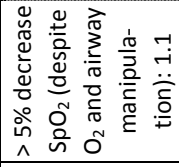 & 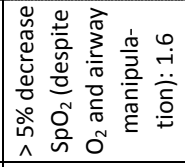 & 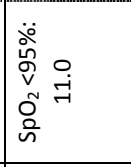 & 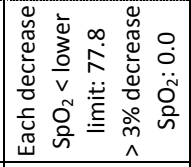 & 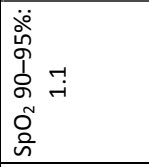 & $\stackrel{\circ}{i}$ \\
\hline$\frac{⿱ ㇒}{z}$ & 孚 & 愛 & $\stackrel{\circ}{\leftrightarrow}$ & $\begin{array}{l}\stackrel{9}{6} \\
\end{array}$ & 魚 & $\frac{o}{z}$ \\
\hline 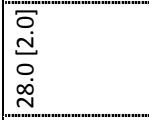 & $\frac{⿱}{z}$ & $\stackrel{m}{r}$ & 品 & $\hat{\grave{\omega}}$ & $\stackrel{m}{0}$ & $\stackrel{\circ}{i}$ \\
\hline $\begin{array}{l}\stackrel{5}{\varepsilon} \\
\stackrel{9}{G} \\
\stackrel{+}{+1}\end{array}$ & 受 & $\frac{\alpha}{z}$ & $\begin{array}{l}\stackrel{9}{+} \\
\stackrel{+1}{m}\end{array}$ & $\frac{\alpha}{z}$ & 品怘离 & $\frac{\mathscr{c}}{z}$ \\
\hline 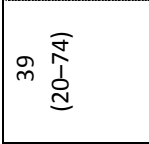 & 受 & 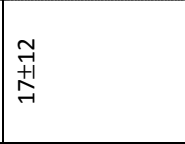 & 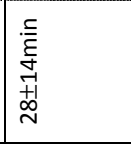 & $\begin{array}{l}+ \\
\infty \\
\infty \\
0 \\
\infty \\
\rightarrow\end{array}$ & 壳 & 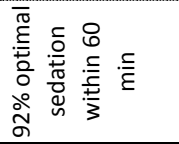 \\
\hline 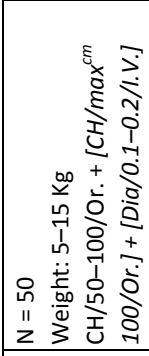 & 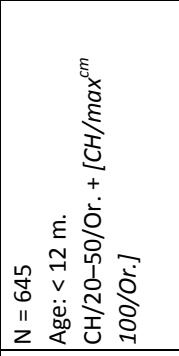 & 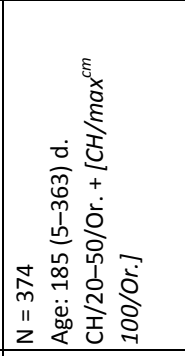 & 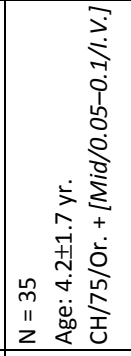 & 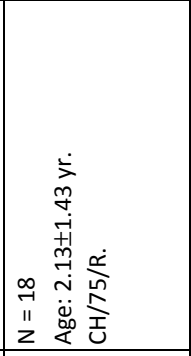 & 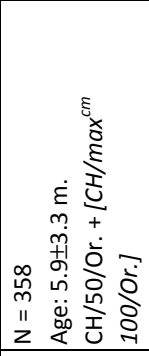 & 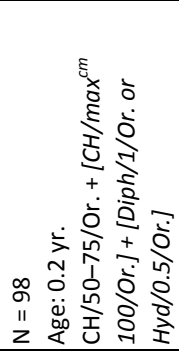 \\
\hline 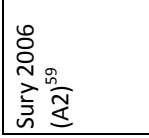 & 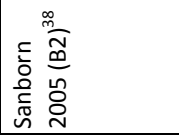 & 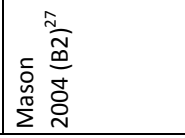 & 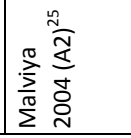 & 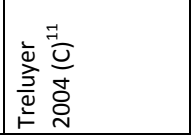 & 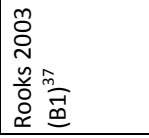 & 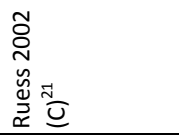 \\
\hline
\end{tabular}




\begin{tabular}{|c|c|c|c|c|c|c|c|}
\hline \multirow{4}{*}{\multicolumn{2}{|c|}{ 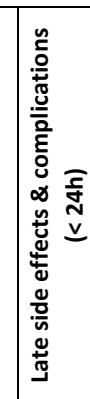 }} & 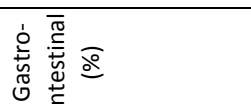 & 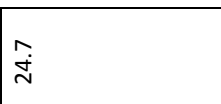 & $\frac{\infty}{z}$ & \multirow{4}{*}{ 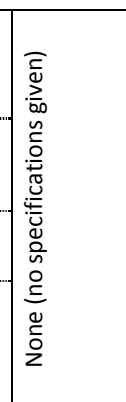 } & $\frac{\infty}{z}$ & $\begin{array}{l}n \\
+1\end{array}$ \\
\hline & & 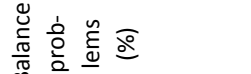 & $\underset{\sim}{\tilde{m}}$ & \multirow{2}{*}{\begin{tabular}{|l|}
$\frac{\alpha}{2}$ \\
$\frac{\alpha}{z}$
\end{tabular}} & & \multirow[t]{2}{*}{$\frac{\infty}{2}$} & $\infty$ \\
\hline & & 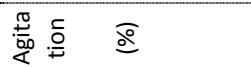 & $\stackrel{\sim}{N}$ & & & & I \\
\hline & & 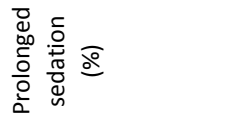 & $\frac{\alpha}{z}$ & $\frac{\infty}{z}$ & & $\frac{\infty}{z}$ & 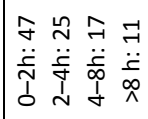 \\
\hline \multirow{6}{*}{\multicolumn{2}{|c|}{ 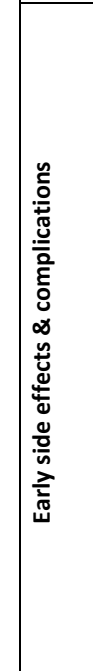 }} & 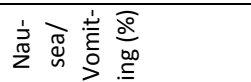 & $\stackrel{4}{4}$ & $\frac{\alpha}{z}$ & \multirow{6}{*}{ 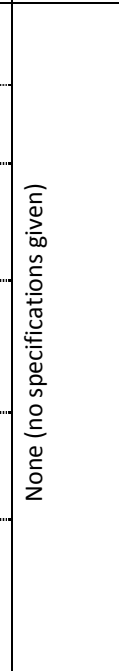 } & $\underset{\sim}{\infty}$ & $\frac{\alpha}{z}$ \\
\hline & & 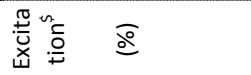 & $\stackrel{i}{4}$ & $\frac{\alpha}{z}$ & & 8 & $\frac{\alpha}{z}$ \\
\hline & & 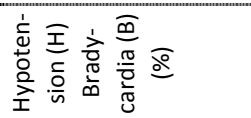 & $\frac{\alpha}{z}$ & $\frac{\alpha}{z}$ & & $\frac{\infty}{2}$ & $\frac{\alpha}{z}$ \\
\hline & & 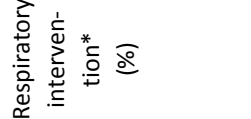 & $\frac{\alpha}{z}$ & $\stackrel{\circ}{\rightarrow}$ & & 8 & : \\
\hline & & 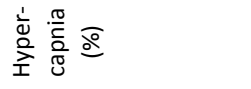 & $\frac{\alpha}{z}$ & $\frac{\alpha}{z}$ & & $\frac{\infty}{z}$ & $\frac{\alpha}{z}$ \\
\hline & & 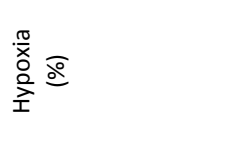 & 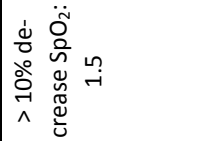 & $\stackrel{9}{\mathrm{~N}}$ & & 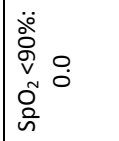 & 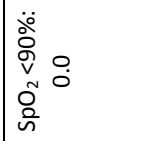 \\
\hline \multirow{4}{*}{ 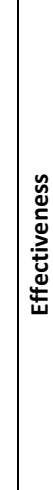 } & & 莺 & $\frac{\alpha}{z}$ & 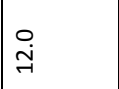 & $\frac{\alpha}{z}$ & $\frac{\infty}{z}$ & $\frac{\alpha}{z}$ \\
\hline & $\check{q}$ & 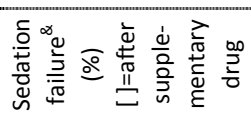 & 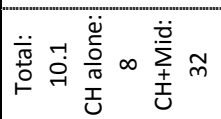 & m & io & $\begin{array}{l}\sigma \\
0 \\
\\
0 \\
\sigma\end{array}$ & 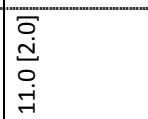 \\
\hline & $\begin{array}{l}\text { 莣 } \\
\text { 离 }\end{array}$ & 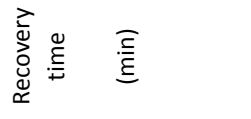 & $\frac{\alpha}{z}$ & $\begin{array}{l}\infty \\
\infty \\
\infty \\
+1 \\
\infty \\
\infty \\
\infty \\
\sim\end{array}$ & $\frac{\alpha}{z}$ & $\frac{\infty}{2}$ & $\frac{\alpha}{z}$ \\
\hline & & 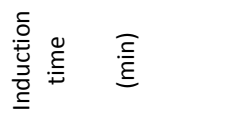 & $\frac{\alpha}{z}$ & 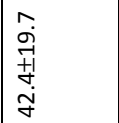 & $\begin{array}{l}m \\
\stackrel{m}{+1} \\
\text { g }\end{array}$ & $\frac{\infty}{2}$ & $\frac{\alpha}{z}$ \\
\hline \multicolumn{3}{|c|}{ 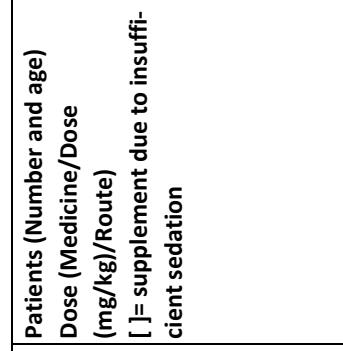 } & 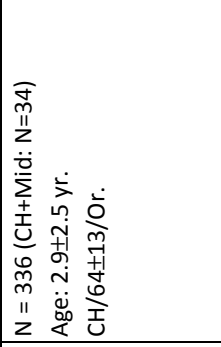 & 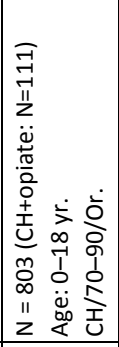 & 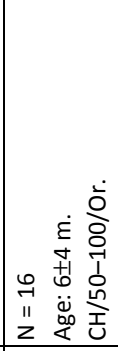 & 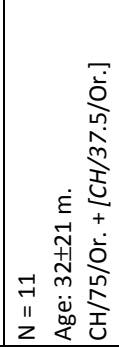 & 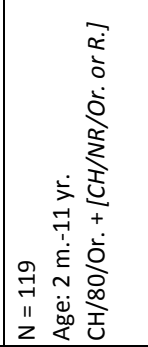 \\
\hline \multicolumn{3}{|c|}{ 誉 } & 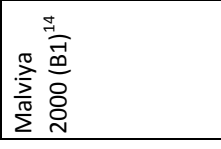 & 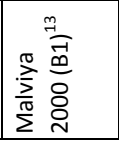 & 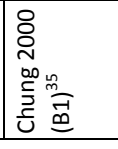 & 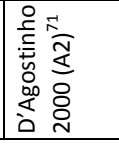 & 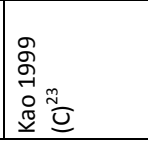 \\
\hline
\end{tabular}




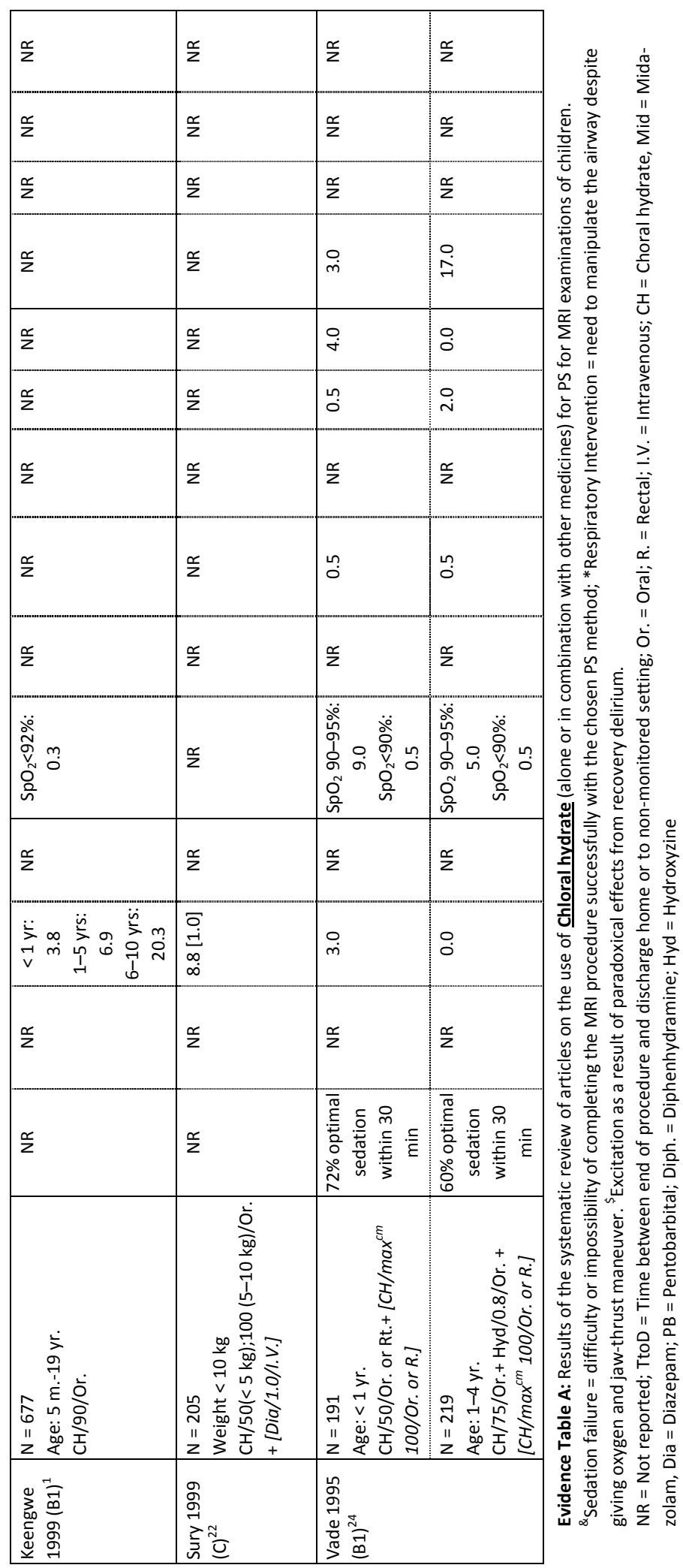




\begin{tabular}{|c|c|c|c|c|c|c|c|c|}
\hline \multirow{4}{*}{\multicolumn{2}{|c|}{ 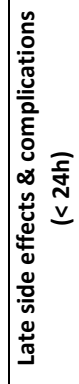 }} & 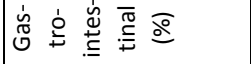 & $\frac{\alpha}{z}$ & $\frac{\alpha}{z}$ & $\frac{\alpha}{z}$ & $\stackrel{n}{\sim}$ & $\frac{\infty}{z}$ & $\frac{\alpha}{z}$ \\
\hline & & 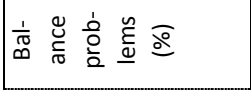 & $\frac{\infty}{z}$ & $\frac{\infty}{z}$ & $\frac{⿱}{z}$ & $\stackrel{n}{\pi}$ & $\frac{\infty}{z}$ & $\frac{\alpha}{z}$ \\
\hline & & 噌 & $\frac{\kappa}{z}$ & $\frac{\alpha}{z}$ & $\frac{\kappa}{z}$ & $\stackrel{n}{r}$ & $\stackrel{\circ}{\circ}$ & $\frac{\alpha}{2}$ \\
\hline & & 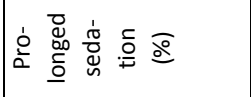 & $\frac{\infty}{z}$ & $\frac{x}{z}$ & $\frac{\alpha}{z}$ & $\frac{\infty}{z}$ & $\frac{\infty}{z}$ & $\frac{\alpha}{z}$ \\
\hline \multirow{6}{*}{\multicolumn{2}{|c|}{ 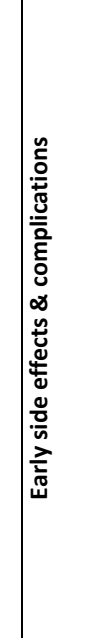 }} & 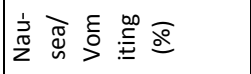 & m & g & $\frac{\kappa}{z}$ & $\because$ & \multirow{6}{*}{ - } & $\stackrel{n}{q}$ \\
\hline & & 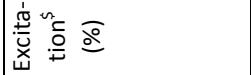 & $\frac{\alpha}{2}$ & $\frac{\infty}{z}$ & $\frac{x}{z}$ & $\stackrel{n}{\sim}$ & & $\frac{\kappa}{z}$ \\
\hline & & 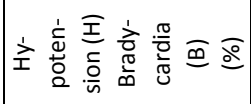 & $\mid \begin{array}{ll}0 & 0 \\
0 & 0 \\
\ddot{x} & 0\end{array}$ & $\mid \begin{array}{ll}0 & 0 \\
0 & 0 \\
\ddot{I} & \ddot{\varphi}\end{array}$ & $\begin{array}{ll}0 & 0 \\
0 & 0 \\
\ddot{I} & \ddot{\theta}\end{array}$ & $\frac{\alpha}{z}$ & & $\frac{x}{z}$ \\
\hline & & 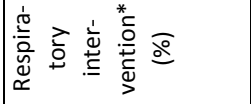 & $0_{0}^{\circ}$ & $:$ & $\frac{x}{z}$ & $\frac{\alpha}{z}$ & & $\because$ \\
\hline & & 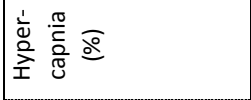 & $\frac{\alpha}{2}$ & $\frac{\alpha}{z}$ & $\frac{x}{z}$ & $\frac{\alpha}{z}$ & & $\frac{\kappa}{z}$ \\
\hline & & 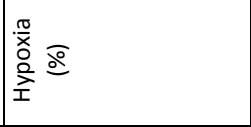 & $\stackrel{+}{\sim}$ & 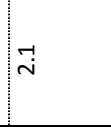 & 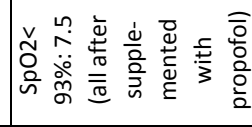 & น & & $\ddot{\circ}$ \\
\hline \multirow{4}{*}{\multicolumn{2}{|c|}{ 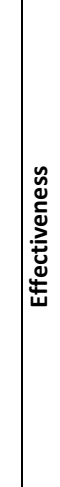 }} & 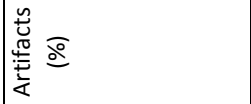 & $\frac{\infty}{2}$ & $\frac{\infty}{z}$ & $\frac{⿱}{z}$ & $\frac{\kappa}{2}$ & $\frac{x}{z}$ & $\frac{\alpha}{z}$ \\
\hline & & 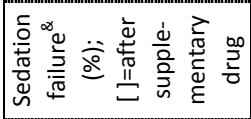 & 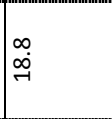 & i & 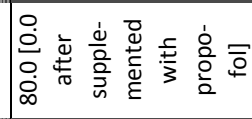 & i̊ & 焉 & i \\
\hline & & 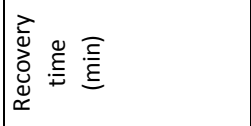 & $\mid \begin{array}{l}\infty \\
+1 \\
\infty \\
\sim \\
\sim\end{array}$ & 䓪 & $\begin{array}{l}0 \\
\ddot{m} \\
\vdots \\
0 \\
\dot{\omega} \\
\dot{\omega}\end{array}$ & $\frac{\alpha}{z}$ & $\frac{\alpha}{z}$ & $\frac{\alpha}{z}$ \\
\hline & & 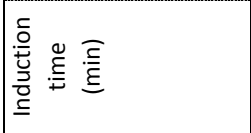 & | & $\frac{1}{2}$ & 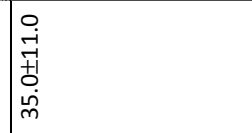 & $\frac{\alpha}{z}$ & $\frac{\alpha}{z}$ & $\frac{\alpha}{z}$ \\
\hline \multicolumn{3}{|c|}{ 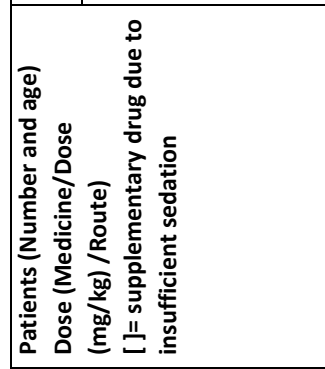 } & 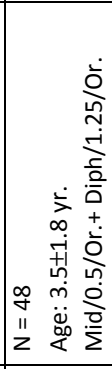 & 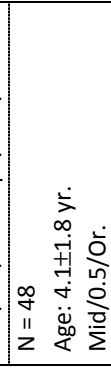 & 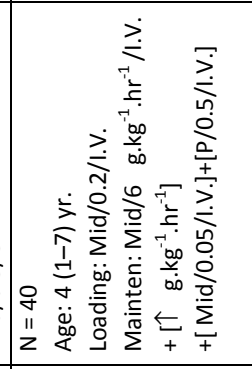 & 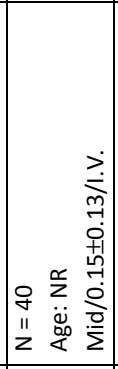 & 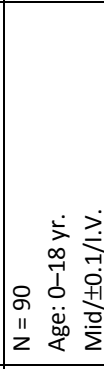 & 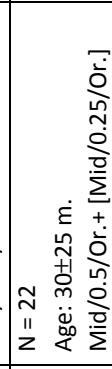 \\
\hline 党. & 章 & & 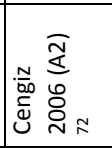 & & 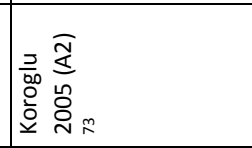 & 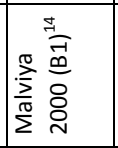 & 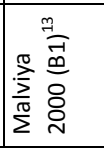 & 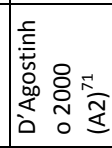 \\
\hline
\end{tabular}




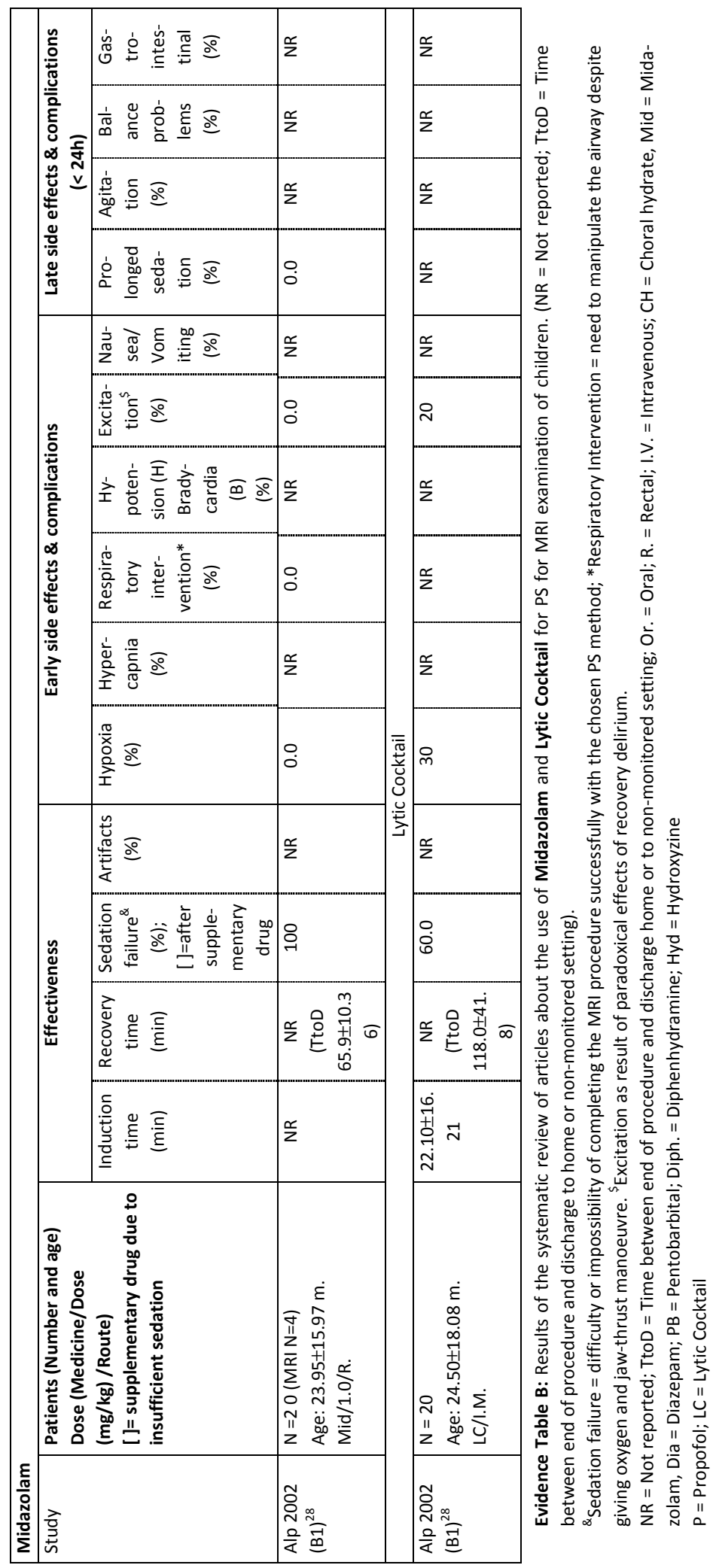




\section{c. Systematic review of published studies on sedatives frequently used in the Netherlands for MRI in children (Evidence tables A and B)}

A total of 23 articles were found, of which 20 concerned the use of chloral hydrate (CH). Only one study focused on the rectal administration of $\mathrm{CH}^{11}$ Six articles described study results for midazolam (MID) and one described study results for a lytic cocktail (LC). No literature was found on pethidine and antihistamines.

\section{Effectiveness}

The incidence of sedation failure for $\mathrm{CH}$ varies strongly between the studies (from 0 to $46 \%$ ). This is primarily the result of divergent definitions. Well-conceived studies suggest that PS using $\mathrm{CH}$ fails in approximately 10 to $30 \%$ of MRI scans. By supplementing the medication with additional $\mathrm{CH}$ and/or other sedatives (usually a benzodiazepine), the rate of sedation failure usually falls to below $5 \%$. Then again, Malviya demonstrated that the addition of MID is associated with a significantly increased risk of sedation failure (up to $32 \%$ ). ${ }^{12}$ The only study focusing on the rectal administration of $\mathrm{CH}$ reported a sedation failure of $16,7 \% .{ }^{11}$ Only a few studies into the use of $\mathrm{CH}$ used the quality of the MRI images as a result criterion. Signs of relevant movement occurred in 4 to $22,5 \%$ of cases ${ }^{11,13-15}$. In comparison with $\mathrm{CH}$, MID and LC are characterized by a high risk of sedation failure (i.e. between 20 and 100\%).

$\mathrm{CH}$ has the greatest effectiveness in infants. The risk of sedation failure rises sharply above the age of one. ${ }^{12,16}$ Behavioural problems and learning difficulties are other notable risk factors for sedation failure. ${ }^{17,18}$ However, the deployment of professionals with specific training who use established sedation protocols leads to higher success rates. ${ }^{1,17,19-22}$

The induction time for PS using CH, MID or LC is characterized by high averages and standard deviations. This means that it can be difficult to synchronize the optimal sedation depth with the time of the examination. The study by Ruess noted a delay in $35 \%$ of cases. ${ }^{21}$ Some studies also measured the recovery time or the time until discharge. $\mathrm{CH}$ and $\mathrm{LC}$ in particular were characterized by a long recovery time. $\mathrm{CH}$ was often associated with a prolonged sedation lasting for many hours after the actual procedure. ${ }^{23,24}$. In Malviya's study, sedation lasted for an average of $11 \pm 10$ hours before the child had recovered to the initial state. ${ }^{25}$

Safety

Most studies describe a comparatively small number of cases $(\mathrm{N}<1000)$. As a result, they lack the power to detect rare (serious) side effects. ${ }^{26}$ In addition, the studies apply very divergent definitions for side effects and complications. The main early complications are desaturations and airway obstructions. These complications are generally mild, but serious incidents do occur. In our systematic review, we found only one reanimation out of a total of 7110 sedations. ${ }^{27}$ This is in line with the 1 in 10,000 risk of serious incidents during PS reported elsewhere. ${ }^{26}$ Airway interventions and/or assisted breathing are required in 0 to $4.6 \%$ of cases. Not one study reported the use of capnography. The incidence of hypoventilation and airway obstruction is undoubtedly underestimated as a result. Serious respiratory incidents occur more frequently with PS using CH or MID in comparison with general anaesthesia. ${ }^{1,13} 28$ In the study by Homan, the use of $\mathrm{CH}$ seemed to be an independent risk factor for complications. ${ }^{29}$

The use of $\mathrm{CH}$ with very young or ex-preterm children is characterized by a high risk of relevant complications such as hypoxia and bradycardia. ${ }^{30}$ These may occur up to several hours after the actual procedure. ${ }^{31}$ The only study that examined the use of LC for MRI reported a high incidence of respiratory complications (30\%). 
Important late side effects of $\mathrm{CH}$ are agitation or irritability (0.5-29\%), balance problems (32$68 \%)$, and gastro-intestinal complaints (0.3-27\%). ${ }^{14,23,25,27}$

\section{d. Systematic review of studies on medications rarely used by Dutch paediatricians for PS during MRI for children}

\section{Barbiturates}

Four studies ( $N=1100$ procedures) describe the use of rectally administered thiopental (TP; dosage $25-50 \mathrm{mg} / \mathrm{kg}$ ). All are of a moderate quality. $\left(\mathrm{B}^{28} ; \mathrm{C}^{32-34}\right)$ In comparison with $\mathrm{CH}$, the induction time appears shorter (on average between 7 and 25 minutes). Sedation failure occurred in 4 to $7 \%$ of cases. The studies do not give information about delays, the quality of the MRI images, or the recovery time. The incidence of mild desaturations varied between 2 and $10 \%$. No serious incidents were reported. Late adverse effects occurred frequently: long-term sedation (13.6\%), balance problems (12.7\%), and gastro-intestinal complaints. ${ }^{33}$

Five studies ( $N=4720$ procedures) were found regarding oral pentobarbital (PB) $\left(B 1{ }^{35-37}\right.$; $\left.B 2{ }^{27,}{ }^{38}\right)$. All studies relate to children under one year old. It is not clear whether the studies are independent from each other. A high success rate $(97.4-99.7 \%)$ is achieved with a total dosage of between 4 and $8 \mathrm{mg} / \mathrm{kg}$. The studies do not report any data regarding delays or the quality of the MRI images. The induction time resembled that of $\mathrm{CH}$. The recovery time was not described separately but the total sedation period was long (on average $100 \pm 35$ minutes). Mild desaturations, airway interventions, and late adverse effects were rare $(<1 \%)$, but were not always systematically investigated. There are indications that in infants, oral PB is just as effective as $\mathrm{CH}$, but is associated less frequently with respiratory complications. ${ }^{27}$

Ten independent studies about intravenous $P B$ were found $\left(N=16447\right.$ procedures) $\left(A 2^{25,39}\right.$; $\left.B 1^{36,40,41} ; B 2{ }^{15,38,42} ; C^{43,44}\right)$. The doses varied between 2 and $9 \mathrm{mg} / \mathrm{kg}$. Sometimes PB was combined with midazolam and/or fentanyl. Reported success rates varied between 88 and $100 \%$. One study reported that movements occurred in $15 \%$ of cases. ${ }^{25}$ Mason et al. described a success rate of $99.7 \%$ in babies under one year old. ${ }^{36}$ The risk of sedation failure was significantly higher above the age of one. ${ }^{44}$ There are indications that the addition of fentanyl increases the success rate to almost $100 \%{ }^{39,}{ }^{42,}{ }^{43}$ Malviya et al. compared intravenous PB with oral $\mathrm{CH}$. Although PB was characterized by a faster onset of sedation (on average $9 \pm 6$ minutes) and less need for supplementary medication, the overall success rate was the same. ${ }^{25}$ Other studies also suggest that the average induction time (9-25 minutes) is shorter than that for $\mathrm{CH}$ or oral $\mathrm{PB} .{ }^{15}$ $36,39,41$ Then again, the average recovery time (35-120 minutes) is comparatively long ${ }^{15,25,36,39-}$ ${ }^{41}$, with recovery to the initial state requiring on average $17 \pm 13$ hours. ${ }^{25}$

Mild desaturations or airway interventions were rare (0.2-1.0\%). Malviya et al. report an incidence of $17 \%$ of mild desaturations and $2.9 \%$ desaturations $<90 \% .{ }^{25} \mathrm{~A}$ relevant early complication, particular with older children, was paradoxical excitation (6.7-14\%). Prolonged sedation $(20 \%)$, agitation (26\%), motor instability (85\%), and gastrointestinal side effects (18\%) are relevant late adverse effects. ${ }^{25,39}$

\section{Ketamine}

Ketamine is a dissociative anaesthetic with a strong analgesic and sedative effect. Since ketamine interferes little with respiration and airway reflexes, it is an interesting medicine for PS. Only one small study was found about PS using ketamine for MRI in children. Haeseler et al. compared the combination of midazolam and $\mathrm{S}(+)$-ketamine with general anaesthesia in a ran- 
domized controlled trial $(\mathrm{N}=34) .{ }^{45}$ The authors concluded that in comparison with anaesthesia, the effectiveness (100\%) and recovery time (average of $44.6 \pm 35.1$ minutes) were the same and the induction time was shorter.

\section{Propofol}

Propofol is a fast-acting, short-duration intravenous anaesthetic that can be easily titrated in accordance with sedation requirements. Sixteen studies were found concerning propofol sedation for MRI examinations $\left(A 2^{39,46-52} ; \mathrm{B}^{40,53} ; \mathrm{B} 2{ }^{15} ; \mathrm{C}^{54-58}\right.$ ). They relate to a total of 6908 procedures, of which 5072 come from the multicenter study by Mallory et al. ${ }^{40}$ Usually, a loading dose $(2-3 \mathrm{mg} / \mathrm{kg}$ ) was administered followed by a titrated maintenance infuse (3-15 mg/kg/hour). Incidences of sedation failure varied between 0 and $12 \%$. In Mallory's study, in which $95.3 \%$ of all sedations were performed by non-anaesthesiology professionals who were specifically trained in PS, the sedation failure was $0.81 \%$. A few studies looked at movement artefacts and reported incidences between 0 and $3.7 \%$. The most notable characteristics of propofol are the very short induction time (one to ten minutes, depending on the induction dose) and recovery period (on average between 0 and 30 minutes). Mild desaturation as a result of respiratory depression is the main side effect with an incidence of 0 to $15 \%$. The preventive administration of oxygen reduces the risk of desaturation to below 3\%. In Mallory's study, desaturations were recorded in $1.22 \%$ of the total number of PS, and it became necessary to intervene with a mask and balloon in $0.32 \%$ of cases. Heard et al. $(\mathrm{N}=20)$ described $5 \%$ mild hypercapnia, whereas Cho et al. $(\mathrm{N}=160)$ and Machat et al. $(\mathrm{N}=500)$ did not observe a single instance of hypercapnia. Inadequate sedation and deep desaturation were very rare in Mallory's study, but they occurred more frequently if the PS was performed by a non-anaesthesiologist. The was not the case for the risk of apnoea. ${ }^{40}$ Not a single study mentioned any reanimations, serious complications causing permanent injury, or fatal incidents. Clinically relevant hypotension or bradycardia was not recorded as complications in any of the studies, nor were there any late adverse effects.

\section{Other medicines}

Sury et al. (A2) studied the effectiveness of melatonin as an adjuvant substance in addition to $\mathrm{CH}$ or in addition to a combination of temazepam and droperidol. Melatonin did not appear to increase the effectiveness of the PS. ${ }^{59}$ Seven studies were found concerning the use of intravenous dexmedetomidine, a selective a2 receptor inhibitor with interesting sedative properties and broad safety margins. Since the product is not yet available in the Netherlands, it will not be discussed any further.

\section{Discussion}

Dutch paediatricians infrequently use of anaesthesia for MRI examinations. The most frequently used medicines to perform PS for MRI examinations are chloral hydrate and to a lesser extent midazolam and lytic cocktails. When asked for an estimate of the sedation success, paediatricians reported that their sedation technique was not optimal in 25 to $50 \%$ of cases. Sedation failure, movement artefacts, delays in relation to the planned moment, or a need to interrupt the procedure for additional sedation were the main causes. It is not known how many MRI examinations of children in the Netherlands fail completely or partially, but the estimates are in line with the results of our literature study. The effectiveness of the most frequently used PS 
technique (moderate sedation based on chloral hydrate with or without midazolam) is suboptimal. Although the average success rate can be up to $90 \%$, the opportunity for high-quality images is lower and the individual predictability of success and timing is limited. The latter is particularly due to the long induction time, the unpredictable individual response, and the fact that chloral hydrate is non-titratable. The deployment of a specifically trained sedation team with adequate logistical support nevertheless generates higher success rates. The longer half-life leads to an unpredictably long recovery period, as a result of which the patient always needs to be monitored for a long period after the procedure. Furthermore, late side-effects such as agitation, motor instability, and deviant behaviour, occur quite frequently.

In comparison with chloral hydrate, the sedation success of midazolam is substantially lower. Too little research has been done into the results of sedation using lytic cocktails to draw any well-founded conclusions. The available evidence and the pharmacological characteristics nevertheless suggest moderate sedation success and a very long recovery period. Further, the need for intramuscular administration is considered to be a disadvantage in paediatrics. In relevant literature, there are nevertheless indications of a high sedation success rate when pentobarbital is used. The comparatively short induction time seems to increase the predictability of the onset of sedation, but the long half-life and the high incidence of unpleasant late side effects form significant disadvantages.

The risk and incidence of (very) serious complications when using chloral hydrate, midazolam, cocktails, and barbiturates are low, but nevertheless higher than during general anaesthesia. ${ }^{13}, 28$ Desaturations of the arterial blood and (impending) airway obstructions are not rare. These can nearly always be explained by an unexpected deeper sedation then intended that interferes with respiration and airway reflexes and that is not acknowledged and treated in time. ${ }^{29,60}$ Other risk factors for serious complications, regardless of the sedative used, are: young age or low weight ${ }^{12,30,31,61-63}$, the combination of sedatives an analgesics $29,38,61,62,64$, comorbidity ${ }^{12,30,38,61,62,65}$, mental retardation ${ }^{2,18}$, the omission of a risk assessment ${ }^{5,29}$, the failure to apply generally accepted safety guidelines ${ }^{5,29}$, and inadequate professional competencies. ${ }^{5,66}$ Recent guidelines therefore recommend that extensive precautions are taken when sedatives are used, in terms of patient selection, monitoring (including the use of capnography), professional competencies, and emergency provisions. Research has shown that the average sedation practice within Dutch paediatric wards is substantially different to the norms. ${ }^{10}$

Of all PS medication available in the Netherlands, a deep sedation with propofol appears to be the most effective. In comparison with general anaesthesia, the chance of success is just as high, without the need for intubation. The logistics are also easier, because there is no need for an MRI-proof ventilator. The very short induction time, the ability to titrate real time to individual requirements, the short recovery period, and the absence of inconvenient late adverse effects are considerable advantages. The use of propofol sedation therefore may result in an optimally efficient MRI program. There are indications that this greater effectiveness results in cost reductions. ${ }^{67}$ The intravenous administration and therefore the need for an intravenous access can be a disadvantage but is also mandatory for safety reasons. Furthermore, propofol is a drug used for induction and maintenance of anaesthesia that, if used improperly, can lead in hypoxia quickly and unexpectedly. General paediatricians in the Netherlands do not usually acquire the necessary competencies during their training to administer propofol as a sedative safely and effectively. Recent literature contains reports of several tens of thousands of propofol sedations, carried out by specifically trained non-anaesthesiologists. These professionals achieve the same results as anaesthesiologists in terms of safety and effectiveness. ${ }^{40,61}$ 


\section{Conclusions and recommendations}

The sedatives used by paediatricians in the Netherlands for MRI are less than optimally effective. Their use is associated with an only mediocre effectiveness and efficiency and considerable safety risks when handled by untrained clinicians. It is not known how many examinations partially or completely fail as a result of ineffective PS, and at what cost consequences. Moreover, these medicines are associated with a real risk of complications. Their use must therefore always be combined with extensive precautionary measures and the presence of professionals who can deal with any complications. ${ }^{3}$ A recent systematic review demonstrated that the same preconditions, level of monitoring, and emergency provisions apply for moderate (e.g. caused by chloral hydrate) and deep (e.g. caused by propofol) sedation. ${ }^{66}$ Optimizing these preconditions requires significant investment in training and infrastructure. Although such interventions will increase patient safety, they can only be cost-efficient provided they actually result in optimal effectiveness. ${ }^{67}$ Propofol is the most effective of all sedatives. Propofol can be used safely by competent, well trained non-anaesthesiologists. ${ }^{68}$ In view of the great requirement for adequate PS for other procedures, it is recommended to press ahead with adequate training and the deployment of specific PS professionals in the Netherlands. For as long as these professionals are not available, it is recommended that children who need to be sedated for MRI are referred to an anaesthesiologist. Specific agreements will be needed, both at the national and local level, to optimize the services provided. 


\section{References}

1. Keengwe IN, Hegde S, Dearlove O, Wilson B, Yates RW, Sharples A. Structured sedation programme for magnetic resonance imaging examination in children. Anaesthesia. 1999;54(11):1069-1072.

2. Kannikeswaran N, Mahajan PV, Sethuraman U, Groebe A, Chen X. Sedation medication received and adverse events related to sedation for brain MRI in children with and without developmental disabilities. Paediatric anaesthesia. 2009;19(3):250-256.

3. Krauss B, Green SM. Procedural sedation and analgesia in children. Lancet. 2006;367(9512):766-780.

4. Cote CJ, Karl HW, Notterman DA, Weinberg JA, McCloskey C. Adverse sedation events in pediatrics: analysis of medications used for sedation. Pediatrics. 2000;106(4):633-644.

5. Cote CJ, Notterman DA, Karl HW, Weinberg JA, McCloskey C. Adverse sedation events in pediatrics: a critical incident analysis of contributing factors. Pediatrics. 2000;105(4 Pt 1):805-814.

6. Guidelines for monitoring and management of pediatric patients during and after sedation for diagnostic and therapeutic procedures: addendum. Pediatrics. 2002;110(4):836-838.

7. Practice guidelines for sedation and analgesia by non-anesthesiologists. Anesthesiology. 2002;96(4): 1004-1017.

8. Guideline statement: management of procedure-related pain in children and adolescents. J Paediatr Child Health. 2006;42 Suppl 1:S1-29.

9. Cote $\mathrm{CJ}$, Wilson S. Guidelines for monitoring and management of pediatric patients during and after sedation for diagnostic and therapeutic procedures: an update. Paediatric anaesthesia. 2008;18(1):910.

10. Leroy PL, Nieman FH, Blokland-Loggers HE, Schipper DM, Zimmermann L, Knape JT. Adherence to safety guidelines on paediatric procedural sedation: the results of a nationwide survey under general paediatricians in The Netherlands. Archives of disease in childhood. 2010.

11. Treluyer JM, Andre C, Carp PF, Chalumeau M, Tonnelier S, Cuq C, et al. Sedation in children undergoing CT scan or MRI: effect of time-course and tolerance of rectal chloral hydrate. Fundamental \& clinical pharmacology. 2004;18(3):347-350.

12. Malviya S, Voepel-Lewis T, Tait AR. Adverse events and risk factors associated with the sedation of children by nonanesthesiologists. Anesthesia and analgesia. 1997;85(6):1207-1213.

13. Malviya S, Voepel-Lewis T, Eldevik OP, Rockwell DT, Wong JH, Tait AR. Sedation and general anaesthesia in children undergoing MRI and $\mathrm{CT}$ : adverse events and outcomes. British journal of anaesthesia. 2000;84(6):743-748.

14. Malviya S, Voepel-Lewis T, Prochaska G, Tait AR. Prolonged recovery and delayed side effects of sedation for diagnostic imaging studies in children. Pediatrics. 2000;105(3):E42.

15. Dalal PG, Murray D, Cox T, McAllister J, Snider R. Sedation and anesthesia protocols used for magnetic resonance imaging studies in infants: provider and pharmacologic considerations. Anesthesia and analgesia. 2006;103(4):863-868.

16. Greenberg SB, Faerber EN, Aspinall CL, Adams RC. High-dose chloral hydrate sedation for children undergoing MR imaging: safety and efficacy in relation to age. Ajr. 1993;161(3):639-641.

17. Beebe DS, Tran P, Bragg M, Stillman A, Truwitt C, Belani KG. Trained nurses can provide safe and effective sedation for $\mathrm{MRI}$ in pediatric patients. Canadian journal of anaesthesia = Journal canadien d'anesthesie. 2000;47(3):205-210.

18. Cortellazzi P, Lamperti M, Minati L, Falcone C, Pantaleoni C, Caldiroli D. Sedation of neurologically impaired children undergoing MRI: a sequential approach. Paediatric anaesthesia. 2007;17(7):630-636.

19. Egelhoff JC, Ball WS, Jr., Koch BL, Parks TD. Safety and efficacy of sedation in children using a structured sedation program. Ajr. 1997;168(5):1259-1262.

20. Karian VE, Burrows PE, Zurakowski D, Connor L, Poznauskis L, Mason KP. The development of a pediatric radiology sedation program. Pediatric radiology. 2002;32(5):348-353.

21. Ruess L, O'Connor SC, Mikita CP, Creamer KM. Sedation for pediatric diagnostic imaging: use of pediatric and nursing resources as an alternative to a radiology department sedation team. Pediatric radiology. 2002;32(7):505-510. 
22. Sury MR, Hatch DJ, Deeley T, Dicks-Mireaux C, Chong WK. Development of a nurse-led sedation service for paediatric magnetic resonance imaging. Lancet. 1999;353(9165):1667-1671.

23. Kao SC, Adamson SD, Tatman LH, Berbaum KS. A survey of post-discharge side effects of conscious sedation using chloral hydrate in pediatric CT and MR imaging. Pediatric radiology. 1999;29(4):287-290.

24. Vade A, Sukhani R, Dolenga M, Habisohn-Schuck C. Chloral hydrate sedation of children undergoing CT and MR imaging: safety as judged by American Academy of Pediatrics guidelines. Ajr. 1995;165(4):905909.

25. Malviya S, Voepel-Lewis T, Tait AR, Reynolds PI, Gujar SK, Gebarski SS, et al. Pentobarbital vs chloral hydrate for sedation of children undergoing MRI: efficacy and recovery characteristics. Paediatric anaesthesia. 2004;14(7):589-595.

26. Cravero JP, Blike GT. Review of pediatric sedation. Anesthesia and analgesia. 2004;99(5):1355-1364.

27. Mason KP, Sanborn P, Zurakowski D, Karian VE, Connor L, Fontaine PJ, et al. Superiority of pentobarbital versus chloral hydrate for sedation in infants during imaging. Radiology. 2004;230(2):537-542.

28. Alp H, Orbak Z, Guler I, Altinkaynak S. Efficacy and safety of rectal thiopental, intramuscular cocktail and rectal midazolam for sedation in children undergoing neuroimaging. Pediatr Int. 2002;44(6):628-634.

29. Hoffman GM, Nowakowski R, Troshynski TJ, Berens RJ, Weisman SJ. Risk reduction in pediatric procedural sedation by application of an American Academy of Pediatrics/American Society of Anesthesiologists process model. Pediatrics. 2002;109(2):236-243.

30. Litman RS, Soin K, Salam A. Chloral hydrate sedation in term and preterm infants: an analysis of efficacy and complications. Anesthesia and analgesia.110(3):739-746.

31. Allegaert K, Naulaers G. Procedural sedation of neonates with chloral hydrate: a sedation procedure does not end at the end of the acquisition of the images. Paediatric anaesthesia. 2008;18(12):12701271.

32. Beekman RP, Hoorntje TM, Beek FJ, Kuijten RH. Sedation for children undergoing magnetic resonance imaging: efficacy and safety of rectal thiopental. European journal of pediatrics. 1996;155(9):820-822.

33. Glasier CM, Stark JE, Brown R, James CA, Allison JW. Rectal thiopental sodium for sedation of pediatric patients undergoing MR and other imaging studies. Ajnr. 1995;16(1):111-114.

34. Nguyen MT, Greenberg SB, Fitzhugh KR, Glasier CM. Pediatric imaging: sedation with an injection formulation modified for rectal administration. Radiology. 2001;221(3):760-762.

35. Chung T, Hoffer FA, Connor L, Zurakowski D, Burrows PE. The use of oral pentobarbital sodium (Nembutal) versus oral chloral hydrate in infants undergoing CT and MR imaging--a pilot study. Pediatric radiology. 2000;30(5):332-335.

36. Mason KP, Zurakowski D, Connor L, Karian VE, Fontaine PJ, Sanborn PA, et al. Infant sedation for MR imaging and CT: oral versus intravenous pentobarbital. Radiology. 2004;233(3):723-728.

37. Rooks VJ, Chung T, Connor L, Zurakowski D, Hoffer FA, Mason KP, et al. Comparison of oral pentobarbital sodium (nembutal) and oral chloral hydrate for sedation of infants during radiologic imaging: preliminary results. Ajr. 2003;180(4):1125-1128.

38. Sanborn PA, Michna E, Zurakowski D, Burrows PE, Fontaine PJ, Connor L, et al. Adverse cardiovascular and respiratory events during sedation of pediatric patients for imaging examinations. Radiology. 2005;237(1):288-294.

39. Pershad J, Wan J, Anghelescu DL. Comparison of propofol with pentobarbital/midazolam/fentanyl sedation for magnetic resonance imaging of the brain in children. Pediatrics. 2007;120(3):e629-636.

40. Mallory MD, Baxter AL, Kost SI. Propofol vs pentobarbital for sedation of children undergoing magnetic resonance imaging: results from the Pediatric Sedation Research Consortium. Paediatric anaesthesia. 2009;19(6):601-611.

41. Mason KP, Zurakowski D, Karian VE, Connor L, Fontaine PJ, Burrows PE. Sedatives used in pediatric imaging: comparison of IV pentobarbital with IV pentobarbital with midazolam added. Ajr. 2001; 177(2):427-430.

42. Ross AK, Hazlett HC, Garrett NT, Wilkerson C, Piven J. Moderate sedation for MRI in young children with autism. Pediatric radiology. 2005;35(9):867-871. 
43. Connor L, Burrows PE, Zurakowski D, Bucci K, Gagnon DA, Mason KP. Effects of IV pentobarbital with and without fentanyl on end-tidal carbon dioxide levels during deep sedation of pediatric patients undergoing MRI. Ajr. 2003;181(6):1691-1694.

44. Greenberg SB, Adams RC, Aspinall CL. Initial experience with intravenous pentobarbital sedation for children undergoing $\mathrm{MRI}$ at a tertiary care pediatric hospital: the learning curve. Pediatric radiology. 2000;30(10):689-691.

45. Haeseler G, Zuzan O, Kohn G, Piepenbrock S, Leuwer M. Anaesthesia with midazolam and S-(+)-ketamine in spontaneously breathing paediatric patients during magnetic resonance imaging. Paediatric anaesthesia. 2000;10(5):513-519.

46. Bryan YF, Hoke LK, Taghon TA, Nick TG, Wang Y, Kennedy SM, et al. A randomized trial comparing sevoflurane and propofol in children undergoing MRI scans. Paediatric anaesthesia. 2009;19(7):672-681.

47. Cho JE, Kim WO, Chang DJ, Choi EM, Oh SY, Kil HK. Titrated propofol induction vs. continuous infusion in children undergoing magnetic resonance imaging. Acta anaesthesiologica Scandinavica.54(4):453-457.

48. Gutmann A, Pessenbacher K, Gschanes A, Eggenreich U, Wargenau M, Toller W. Propofol anesthesia in spontaneously breathing children undergoing magnetic resonance imaging: comparison of two propofol emulsions. Paediatric anaesthesia. 2006;16(3):266-274.

49. Heard C, Burrows F, Johnson K, Joshi P, Houck J, Lerman J. A comparison of dexmedetomidinemidazolam with propofol for maintenance of anesthesia in children undergoing magnetic resonance imaging. Anesthesia and analgesia. 2008;107(6):1832-1839.

50. Koroglu A, Teksan H, Sagir O, Yucel A, Toprak HI, Ersoy OM. A comparison of the sedative, hemodynamic, and respiratory effects of dexmedetomidine and propofol in children undergoing magnetic resonance imaging. Anesthesia and analgesia. 2006;103(1):63-67, table of contents.

51. Shorrab AA, Demian AD, Atallah MM. Multidrug intravenous anesthesia for children undergoing MRI: a comparison with general anesthesia. Paediatric anaesthesia. 2007;17(12):1187-1193.

52. Tomatir E, Atalay H, Gurses E, Erbay H, Bozkurt P. Effects of low dose ketamine before induction on propofol anesthesia for pediatric magnetic resonance imaging. Paediatric anaesthesia. 2004;14(10): 845-850.

53. Amundsen LB, Artru AA, Dager SR, Shaw DW, Friedman S, Sparks B, et al. Propofol sedation for longitudinal pediatric neuroimaging research. Journal of neurosurgical anesthesiology. 2005;17(4):180-192.

54. Levati A, Colombo N, Arosio EM, Savoia G, Tommasino C, Scialfa G, et al. Propofol anaesthesia in spontaneously breathing paediatric patients during magnetic resonance imaging. Acta anaesthesiologica Scandinavica. 1996;40(5):561-565.

55. Tsui BC, Wagner A, Usher AG, Cave DA, Tang C. Combined propofol and remifentanil intravenous anesthesia for pediatric patients undergoing magnetic resonance imaging. Paediatric anaesthesia. 2005; 15(5):397-401.

56. Usher AG, Kearney RA, Tsui BC. Propofol total intravenous anesthesia for MRI in children. Paediatric anaesthesia. 2005;15(1):23-28.

57. Machata AM, Willschke H, Kabon B, Kettner SC, Marhofer P. Propofol-based sedation regimen for infants and children undergoing ambulatory magnetic resonance imaging. British journal of anaesthesia. 2008;101(2):239-243.

58. Hasan RA, Shayevitz JR, Patel V. Deep sedation with propofol for children undergoing ambulatory magnetic resonance imaging of the brain: experience from a pediatric intensive care unit. Pediatr Crit Care Med. 2003;4(4):454-458.

59. Sury MR, Fairweather $\mathrm{K}$. The effect of melatonin on sedation of children undergoing magnetic resonance imaging. British journal of anaesthesia. 2006;97(2):220-225.

60. Motas D, McDermott NB, VanSickle T, Friesen RH. Depth of consciousness and deep sedation attained in children as administered by nonanaesthesiologists in a children's hospital. Paediatric anaesthesia. 2004;14(3):256-260.

61. Cravero JP, Beach ML, Blike GT, Gallagher SM, Hertzog JH. The incidence and nature of adverse events during pediatric sedation/anesthesia with propofol for procedures outside the operating room: a report from the Pediatric Sedation Research Consortium. Anesthesia and analgesia. 2009;108(3):795-804. 
62. Green SM, Roback MG, Krauss B, Brown L, McGlone RG, Agrawal D, et al. Predictors of airway and respiratory adverse events with ketamine sedation in the emergency department: an individual-patient data meta-analysis of 8,282 children. Annals of emergency medicine. 2009;54(2):158-168 e151-154.

63. Resch K, Schilling C, Borchert BD, Klatzko M, Uden D. Topical anesthesia for pediatric lacerations: a randomized trial of lidocaine-epinephrine-tetracaine solution versus gel. Annals of emergency medicine. 1998;32(6):693-697.

64. Pitetti RD, Singh S, Pierce MC. Safe and efficacious use of procedural sedation and analgesia by nonanesthesiologists in a pediatric emergency department. Archives of pediatrics \& adolescent medicine. 2003;157(11):1090-1096.

65. Vespasiano M, Finkelstein M, Kurachek S. Propofol sedation: intensivists' experience with 7304 cases in a children's hospital. Pediatrics. 2007;120(6):e1411-1417.

66. Leroy PL, Schipper DM, Knape HJ. Professional skills and competence for safe and effective procedural sedation in children: recommendations based on a systematic review of the literature. International journal of pediatrics.2010:934298.

67. Kain ZN, Gaal DJ, Kain TS, Jaeger DD, Rimar S. A first-pass cost analysis of propofol versus barbiturates for children undergoing magnetic resonance imaging. Anesthesia and analgesia. 1994;79(6):1102-1106.

68. Krauss B, Green SM. Training and credentialing in procedural sedation and analgesia in children: lessons from the United States model. Paediatric anaesthesia. 2008;18(1):30-35.

69. Beauve B, Dearlove O. Sedation of children under 4 weeks of age for MRI examination. Paediatric anaesthesia. 2008;18(9):892-893.

70. Low $E$, O'Driscoll M, MacEneaney $P, O^{\prime}$ Mahony $O$. Sedation with oral chloral hydrate in children undergoing MRI scanning. Irish medical journal. 2008;101(3):80-82.

71. D'Agostino J, Terndrup TE. Chloral hydrate versus midazolam for sedation of children for neuroimaging: a randomized clinical trial. Pediatric emergency care. 2000;16(1):1-4.

72. Cengiz M, Baysal Z, Ganidagli S. Oral sedation with midazolam and diphenhydramine compared with midazolam alone in children undergoing magnetic resonance imaging. Paediatric anaesthesia. 2006; 16(6):621-626.

73. Koroglu A, Demirbilek S, Teksan H, Sagir O, But AK, Ersoy MO. Sedative, haemodynamic and respiratory effects of dexmedetomidine in children undergoing magnetic resonance imaging examination: preliminary results. British journal of anaesthesia. 2005;94(6):821-824. 


\section{Appendix to Part 2.2}

Successful and safe sedation of children for MRI examination

This section has been published in:

Piet LM Leroy, Hans(J) TA Knape. Archives of Disease in Childhood 2011; Published on line $-10^{\text {th }}$ of February 2011.

(http://adc.bmj.com/content/96/1/114.1.long/reply\#archdischild_el_9150) 


\section{Letter to the editor}

\section{Procedural sedation for paediatric neuroimaging: time to move on!}

In their observational study Sammons et al. showed that general anaesthesia (GA) is more convenient and better tolerated than procedural sedation (PS) for paediatric neuroimaging.[1] These findings are fully consistent with what can be obviously concluded from recent literature: in paediatric neuroimaging, and especially in magnetic resonance imaging, standard sedatives lack optimal effectiveness. The obvious explanation is the unpredictability of onset, depth and duration of sedation. Although the incidence of sedation failure is usually below $10 \%$, delay, motion artefacts, interruption of procedure for supplementary sedation and interference with scanning schedule occur frequently. In addition, the long half-life makes an extensive monitored recovery period imperative, generating an extra burden for health care. Finally, these drugs may cause unexpectedly deep sedation that might interfere with respiratory reflexes.[2] Their use must therefore be restricted to settings with high safety standards for monitoring, professional competences and rescue facilities. From a cost-benefit point-of-view one may question the justifiability of implying these standards in a sedation practice that applies suboptimal sedatives. Simply replacing PS by GA is not a reasonable alternative, given the generally limited anaesthesia services for neuroimaging.

Recent literature yields interesting new concepts. The anaesthetic propofol is an excellent sedative for PS in spontaneously breathing children. Its short induction and recovery times and optimal titratability make propofol a suitable alternative for GA in neuroimaging.[3] Furthermore, there is good evidence that well-trained non-anaesthesiologists may provide propofol sedation safely.[4] Appropriate safety precautions, monitoring and professional skills, rather than professional title, are determinants for its safe and effective use. [5] Time has come to further explore these concepts and to move to practical implementation. Optimally safe and effective PS in paediatric neuroimaging needs competent sedation providers who are specifically trained in deep sedation using highly effective drugs within a context of transparency and ongoing quality control.

\section{References}

1. Sammons, H.M., et al., General anaesthesia or sedation for paediatric neuroimaging: current practice in a teaching hospital. Arch Dis Child. 96(1): p. 114.

2. Motas, D., et al., Depth of consciousness and deep sedation attained in children as administered by nonanaesthesiologists in a children's hospital. Paediatr Anaesth, 2004. 14(3): p. 256-60.

3. Mallory, M.D., A.L. Baxter, and S.I. Kost, Propofol vs pentobarbital for sedation of children undergoing magnetic resonance imaging: results from the Pediatric Sedation Research Consortium. Paediatr Anaesth, 2009. 19(6): p. 601-11.

4. Cravero, J.P., et al., The incidence and nature of adverse events during pediatric sedation/anesthesia with propofol for procedures outside the operating room: a report from the Pediatric Sedation Research Consortium. Anesth Analg, 2009. 108(3): p. 795-804.

5. Green, S.M. and B. Krauss, Barriers to propofol use in emergency medicine. Ann Emerg Med, 2008. 52(4): p. 392-8. 
Table 1 Sedation protocol for scans

\begin{tabular}{lll}
\hline & $<4$ years & $>4$ years \\
\hline 1st Ine drugs & Chloral hydrate $50-100 \mathrm{mg} / \mathrm{kg}(\max 2 \mathrm{~g})$ & $\begin{array}{l}\text { Quinalbarbitone } 7.5-10 \mathrm{mg} / \mathrm{kg} \text { to nearest } 25 \mathrm{mg} \\
\text { (max 200 } \mathrm{mg})\end{array}$ \\
2nd line drugs & $\begin{array}{l}\text { PR Paraldehyde } 0.3 \mathrm{~m} / \mathrm{kg}+\text { equal volume of } \\
\text { ollve oil (max } 12 \mathrm{ml})\end{array}$ & $\begin{array}{l}\text { PR Paraldehyde } 0.3 \mathrm{~m} / \mathrm{kg}+\text { equal volume of olive } \\
\text { oil (max } 12 \mathrm{mll})\end{array}$ \\
\hline
\end{tabular}

Table 2 Opinions of parents and children on the experience of receiving oral sedation or general anaesthesia for neuroimaging

\begin{tabular}{llll}
\hline Parents' experience & OK & 'Bad' or 'quite bad' & Not present \\
\hline Oral sedation, $n=145$ & $128(90 \%)$ & $14(10 \%)$ & 3 \\
GA, $n=69$ & $65(97 \%)$ & $2(3 \%)$ & 2 \\
\hline Children's experience & OK & 'Bad' or 'quite bad' & Too young \\
\hline Oral sedation, $n=172$ & $31(85 \%)$ & $5(15 \%)$ & 136 \\
GA, $n=79$ & $44(98 \%)$ & $1(2 \%)$ & 34 \\
\hline
\end{tabular}

GA, general anaesthesia.

cent of children had neuro-developmental disabilities. Twenty-five per cent had scans before cochlear implant surgery. Airway support was needed for $30 \%$ in recovery, and for one child in transit to the ward. No children vomited during general anaesthesia, but one vomited afterwards and two became nauseated requiring ondansetron. All scans were successful. In 103 children for whom we collected data, median time for the child to wake was $1 \mathrm{~h}$ and $30 \mathrm{~min}$ (IQR $1 \mathrm{~h} 12 \mathrm{~min}$ to 1 h $45 \mathrm{~min}$ ).

Parents and children were asked to grade their experiences (table 2).

Our rates for successful sedated scans were similar to those reported in other studies in the UK. ${ }^{3}$ The one serious adverse event (an unscheduled overnight hospital stay) in 301 sedated scans is consistent with a serious adverse event rate of $0.33 \%$ ( $95 \% \mathrm{Cl} 0.06$ to $1.85 \%$ ).

Although observational, our results suggest that general anaesthesia could be more convenient and better tolerated than sedation for neuroimaging children. Future work to compare the economic cost and patient satisfaction of a mixed service of sedation and anaesthesia versus anaesthesia alone may help determine how can we most efficiently use our current resources and best serve our patients' interests.

\section{H M Sammons, 'J Edwards, ${ }^{2}$ R Rushby.}

C Picton. ${ }^{2} \mathrm{~J}$ Colliet,' W P Whitehouse

'Academic Division of Child Health, The Medical School, Derbyshire Children's Hospital, Derby. UK IUniversity Hospital Nottingham. Nottingham, UK 'Faculty of Medicine and Health Sciences, School of Nursing. Queen's Medical Centre, Nottingham, UK Nursing. Queen's Medical Centre. Nottingham, UK
'University of Nottingham, Queen's Medical Centre, Nottingham, UK

Correspondence to DrW PWhitehouse Department of Paediatric Neurology, Division of Human Development, University of Nottingham and Nottingham University Hospitals NHS Trust, Queen's
Medical Centre, Nottingham NG7 2UH, UK: william.whitehouse@nottingham.ac.uk

Acknowledgements We thank all the MPI sedation nurses, the rurses on ward E39 and Ambulatory Care, and the anaesthetists at the Nottingham University Hospitals NHS Trust, Queen's Medical Centre, Nottingham for thei help in the audit.

\section{Competing interests None.}

Provenance and peer review Not commissioned: externally peer reviewed.

Accepted 23 September 2010 Published Onine First 27 October 2010

Arch Ois Child 2011:96:114.

doi: $10.1136 / a d c: 2010.185256$

\section{REFERENCES}

1. SIGN. Safe Sedation of Children Undergoing Diagnostic and Therapeutic Procedures. A National Clinical Guideline No 58 . Edirburgh: Scottish Intercollegiate Guidelines Network, 2004.

2. Sury MR, Harker $H$, Begent J, et al The management of infants and chlidren for painless imaging Cin Radiol 2005,60:731-41.

3. Woodthorpe C, Trigg A. Alison G, et at. Nurse led sedation for paediatric MRi: progress and issues. Paediatr Nurs2007; 19:14-18 



\section{Part 2.3 Safe and effective procedural sedation/analgesia (PSA) for gastro-intestinal endoscopy in children.}

A systematic review of the literature 


\section{Abstract}

Objective: To assess the safest and most effective way to provide procedural sedation and analgesia (PSA) in children undergoing gastrointestinal endoscopy (GIE).

Study designs and methods: The databases Medline, Cochrane Library and Embase were used. Search terms 'endoscopy, gastrointestinal' or 'endoscopy, digestive system' were combined with 'sedation', 'analgesia', 'conscious sedation', 'moderate sedation', 'deep sedation', 'hypnotics and sedatives'. The final review was restricted to studies reporting specifically on safety (incidences of adverse events) and/or effectiveness (time characteristics, need for supplemental sedation, need for restraint, procedural success, provider satisfaction and patient comfort) of PSA for GIE in children younger than $18 \mathrm{yr}$.

Results: The search yielded 182 references and the final selection included 11 Randomized Controlled Trials (RCT) and 15 non-RCTs. Six sedation categories were identified: propofol-based, opioid/benzodiazepine-based, premedication, ketamine-based, sevoflurane-based and midazolam-based. Only a few RCTs have compared different categories. Opioid/benzodiazepinebased PSA and propofol-based PSA have a similar safety profile with a very low incidence of major adverse events. Propofol-based sedation turned out to be the most effective regimen, with effectiveness comparable to general anaesthesia. The addition of midazolam, fentanyl, remifentanil, and/or ketamine to propofol may increase the effectiveness without creating more adverse events. Data on midazolam-, ketamine- and sevoflurane-based sedation were generally too limited to draw conclusions.

Conclusions: Despite a lack of randomized controlled trials containing all aspects of effectiveness and safety, the current evidence indicates propofol-based PSA to be the best practice for PSA in children undergoing GIE. Propofol can be safely administered by specifically trained nonanaesthesiologists.

Key words: Gastro-intestinal endoscopy, procedural sedation, effectiveness, safety, children 


\section{Introduction}

Gastrointestinal endoscopy (GIE) is a well-established procedure for diagnosis and treatment in paediatric gastroenterology. Despite local anaesthesia, reassurance and distraction techniques most children are unable to undergo GIE without being physically restrained. ${ }^{1,2}$ In addition, the recall of an unpleasant GIE generates a more negative attitude towards future endoscopies. ${ }^{3}$ Therefore, children undergoing GIE will need anaesthesia or procedural sedation (PSA) to guarantee an optimal comfort and cooperation. PSA can be defined as the use of sedative, analgesic, or dissociative drugs to relieve anxiety and pain associated with diagnostic and therapeutic procedures, while maintaining spontaneous ventilation. ${ }^{4,5}$ PSA covers a large spectrum of sedatives and involves a wide range of sedation levels. (Table 1) Light sedation, formerly called anxiolysis, is typically the result of one standard dose of midazolam or by the breathing of $50 \% \mathrm{Ni}$ trous oxide. ${ }^{6}$ Moderate sedation, formerly called conscious sedation, is often used incorrectly to describe a state that is probably more like deep sedation: reflex withdrawal to a painful stimulus alone should not be considered as rousable. ${ }^{7}$ The term deep sedation has been under discussion in some professional groups, because it may be indistinguishable from anaesthesia. While this point may be overstated it has led to the recommendation that the same personnel, equipment and facilities must manage both deep sedation and anaesthesia. Some have proposed other descriptions of deep sedation/anaesthesia: the terms light anaesthesia or minimal anaesthesia may be more appropriate to describe a technique in which the patient seems unconscious although any appreciable stimulation is likely to rouse them. ${ }^{8,9}$

PSA provided in a context of inadequate training, competence or safety precautions is associated with a greater likelihood of potentially fatal complications. ${ }^{4,} 10$ PSA by the untrained also bares the risk of ineffective sedation, possibly leading to unsuccessful and/or uncomfortable procedures. Paediatric gastroenterologists differ in their opinions on the effectiveness of PSA, and many prefer anaesthesiologist's assistance. ${ }^{11}$ Depending on local anaesthesiology resources, traditions and personal experience endoscopists have adopted different PSA techniques. ${ }^{12,13}$ This diversity of sedation practices is, at least partially, the consequence of a lack of consensus on the safest and most effective regimen. By systematically reviewing the literature, we aimed to answer the following clinical question: what is the safest and most effective way to provide PSA in children undergoing GIE?

\section{Methods}

Literature was searched in the databases Medline, Cochrane Library and Embase. The search terms 'endoscopy, gastrointestinal' or 'endoscopy, digestive system' were combined with the terms 'sedation', 'analgesia' 'conscious sedation', 'moderate sedation', 'deep sedation', 'hypnotics and sedatives'. The search was restricted to papers including human subjects aged 0-18 years and published between January 1995 and January 2011. Additional records were searched in bibliographies of published studies, review articles, editorials and guidelines. In a first step papers addressing a mainly adult population were excluded. Next, both authors reviewed independently the full text of the remaining articles. Authors were not blinded for either the journal or authors. Exclusion criteria were: studies not reporting on the safety or effectiveness of PSA; studies focusing on non-GIE procedures; studies on specific endoscopy procedures (e.g. endoscopic retrograde cholangiopancreatography) or specific research populations (e.g. critically ill 
Table 1: Definitions of levels of sedation and significance for the respiratory and cardiovascular condition (American Academy of Paediatrics)

\begin{tabular}{|c|c|c|}
\hline Sedation level & Definition $^{5,40}$ & $\begin{array}{l}\text { Significance for Respiratory and Circulatory } \\
\text { Condition }\end{array}$ \\
\hline Light sedation & $\begin{array}{l}\text { Patients respond normally to verbal commands. } \\
\text { Cognitive function and coordination may be } \\
\text { impaired, }\end{array}$ & $\begin{array}{l}\text { Ventilatory and cardiovascular functions are } \\
\text { unaffected. }\end{array}$ \\
\hline $\begin{array}{l}\text { Moderate } \\
\text { sedation }\end{array}$ & $\begin{array}{l}\text { Patients respond purposefully to verbal com- } \\
\text { mands (e.g., "open your eyes," either alone } \\
\text { or accompanied by light tactile stimulation, } \\
\text { such as a light tap on the shoulder or face, } \\
\text { not a sternal rub). } \\
\text { For older patients, this level of sedation implies } \\
\text { an interactive state; for younger patients, } \\
\text { age appropriate behaviors (e.g., crying) occur } \\
\text { and are expected. } \\
\text { Reflex withdrawal, although a normal response } \\
\text { to a painful stimulus, is not considered as the } \\
\text { only age-appropriate purposeful response } \\
\text { (i.e., it must be accompanied by another re- } \\
\text { sponse, such as pushing away the painful } \\
\text { stimulus, to confirm a higher cognitive func- } \\
\text { tion) }\end{array}$ & $\begin{array}{l}\text { No intervention is required to maintain a patent } \\
\text { airway, and spontaneous ventilation is ade- } \\
\text { quate. Cardiovascular function is usually } \\
\text { maintained. However, in the case of proce- } \\
\text { dures that may themselves cause airway ob- } \\
\text { struction (e.g., dental or endoscopic), the } \\
\text { practitioner must recognize an obstruction } \\
\text { and assist the patient in opening the airway. } \\
\text { If the patient is not making spontaneous efforts } \\
\text { to open their airway to relieve the obstruc- } \\
\text { tion, then the patient should be considered } \\
\text { to be deeply sedated. }\end{array}$ \\
\hline Deep sedation & $\begin{array}{l}\text { Patients cannot be easily aroused but respond } \\
\text { purposefully (see discussion of reflex with- } \\
\text { drawal above) after repeated verbal or pain- } \\
\text { ful stimulation (eg, purposefully pushing } \\
\text { away the noxious stimuli). }\end{array}$ & $\begin{array}{l}\text { The ability to independently maintain ventila- } \\
\text { tory function may be impaired. Patients may } \\
\text { require assistance in maintaining a patent } \\
\text { airway, and spontaneous ventilation may be } \\
\text { inadequate. A state of deep sedation may be } \\
\text { accompanied by partial or complete loss of } \\
\text { protective airway reflexes. } \\
\text { Cardiovascular function is usually maintained. }\end{array}$ \\
\hline Anaesthesia & $\begin{array}{l}\text { Patients are not arousable, even by painful } \\
\text { stimulation. }\end{array}$ & $\begin{array}{l}\text { The ability to independently maintain ventila- } \\
\text { tory function is often impaired. Patients of- } \\
\text { ten require assistance in maintaining a pat- } \\
\text { ent airway, and positive-pressure ventilation } \\
\text { may be required because of depressed spon- } \\
\text { taneous ventilation or drug-induced depres- } \\
\text { sion of neuromuscular function. } \\
\text { Cardiovascular function may be impaired. }\end{array}$ \\
\hline
\end{tabular}

children); review articles; editorials; policy statements; guidelines; case reports. Disagreements were discussed and solved among authors. The final review was restricted to studies that reported specifically on 'safety' and/or 'effectiveness' of PSA for GIE in children. Safety was assessed using incidences of adverse events: hypoxia, hypoventilation or apnea, laryngeal spasm, bradycardia, hypotension, vomiting, complications with fatal outcome or permanent sequelae and the need for rescue interventions. Effectiveness was assessed using time characteristics (induction, recovery and total sedation time), the need for supplemental sedation, procedural success, provider satisfaction and patient comfort (stress or pain scores; recall; parent or patient satisfaction). We defined that an optimally effective PSA technique should achieve near $100 \%$ predictable procedural success and timing, minimal induction and recovery times, and an optimal patient comfort (absence of procedural pain, anxiety or the need for restraint. ${ }^{2}$ 
To answer the clinical question results from randomized controlled trials (RCT) were used at first. If these sources remained inconclusive the results of non-RCTs were used to formulate a deliberate answer. Non-RCTs were classified as B1 for 'comparative-prospective', B2 for 'comparative-retrospective' and C for 'non-comparative' studies.

\section{Results}

The results of the literature search and selection process are summarized in figure 1 . The final selection included 25 papers, reporting on 26 studies (11 RCTs and 15 non-RCTs). One paper included the results from a retrospective comparative study and a RCT. ${ }^{14}$ RCTs are summarized in an evidence table, listing details on methodology, results, limitations and conclusions. (Table 2) Non-RCT studies are summarized in Table 3. Esophago-gastroduodenoscopy (EGD) was the most frequent GIE. Depending on the investigated sedative(s), RCTs can be divided in 3 categories: propofol-based, opioid+benzodiazepine combination and studies on premedication. NonRCT literature yielded three additional categories, i.e. ketamine-based, sevoflurane-based and midazolam-based protocols. Results are ordered according to the number of reported procedures. Studies on premedication are reported separately.

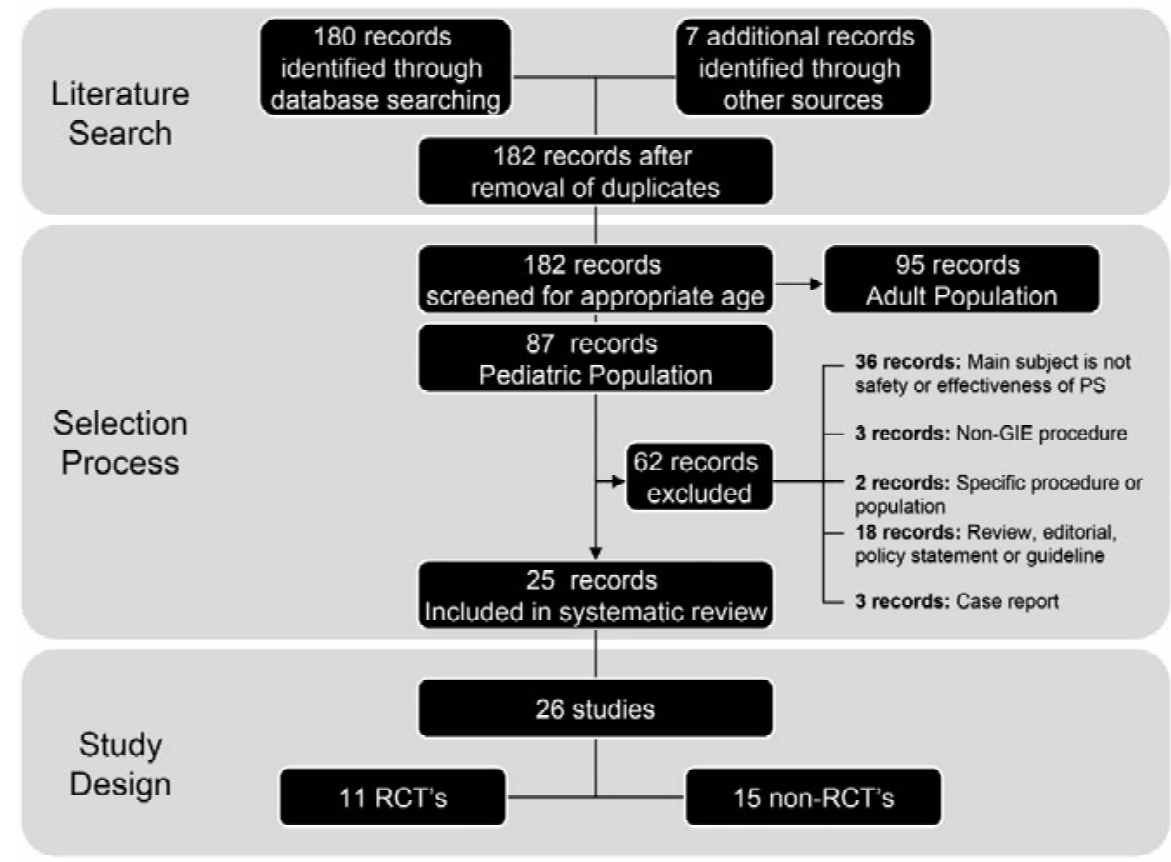

Figure 1: Results of the literature search and selection process 


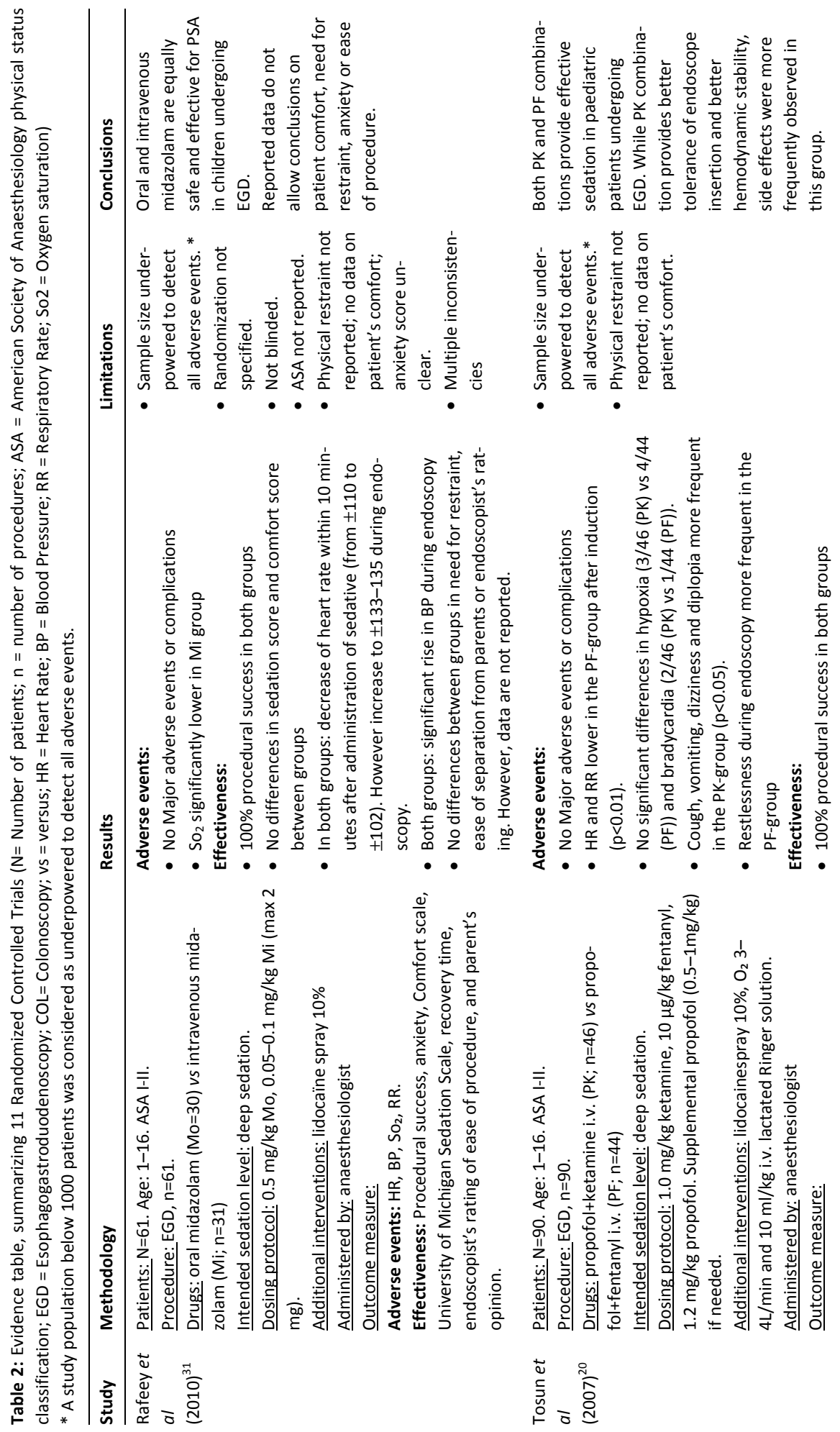




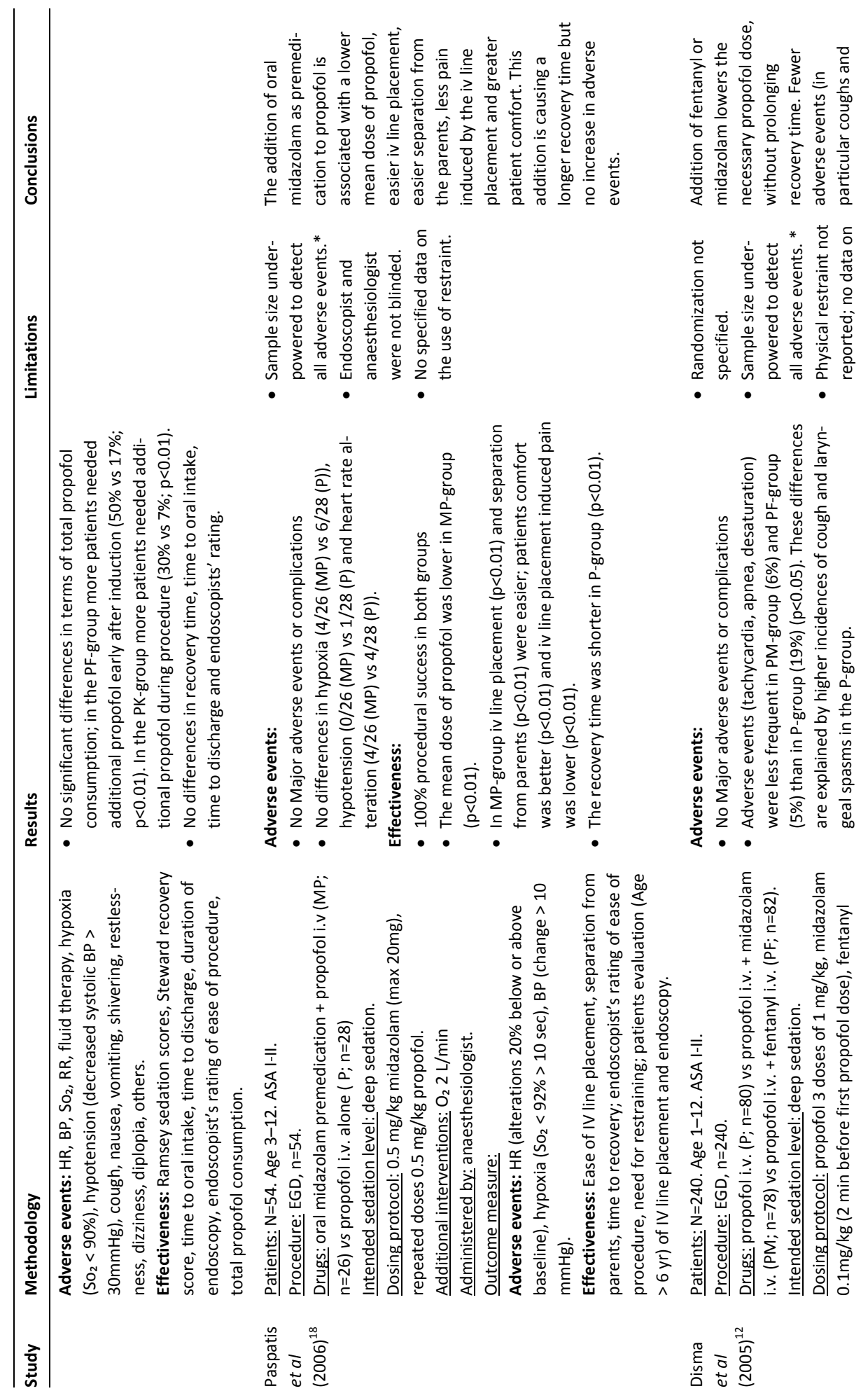




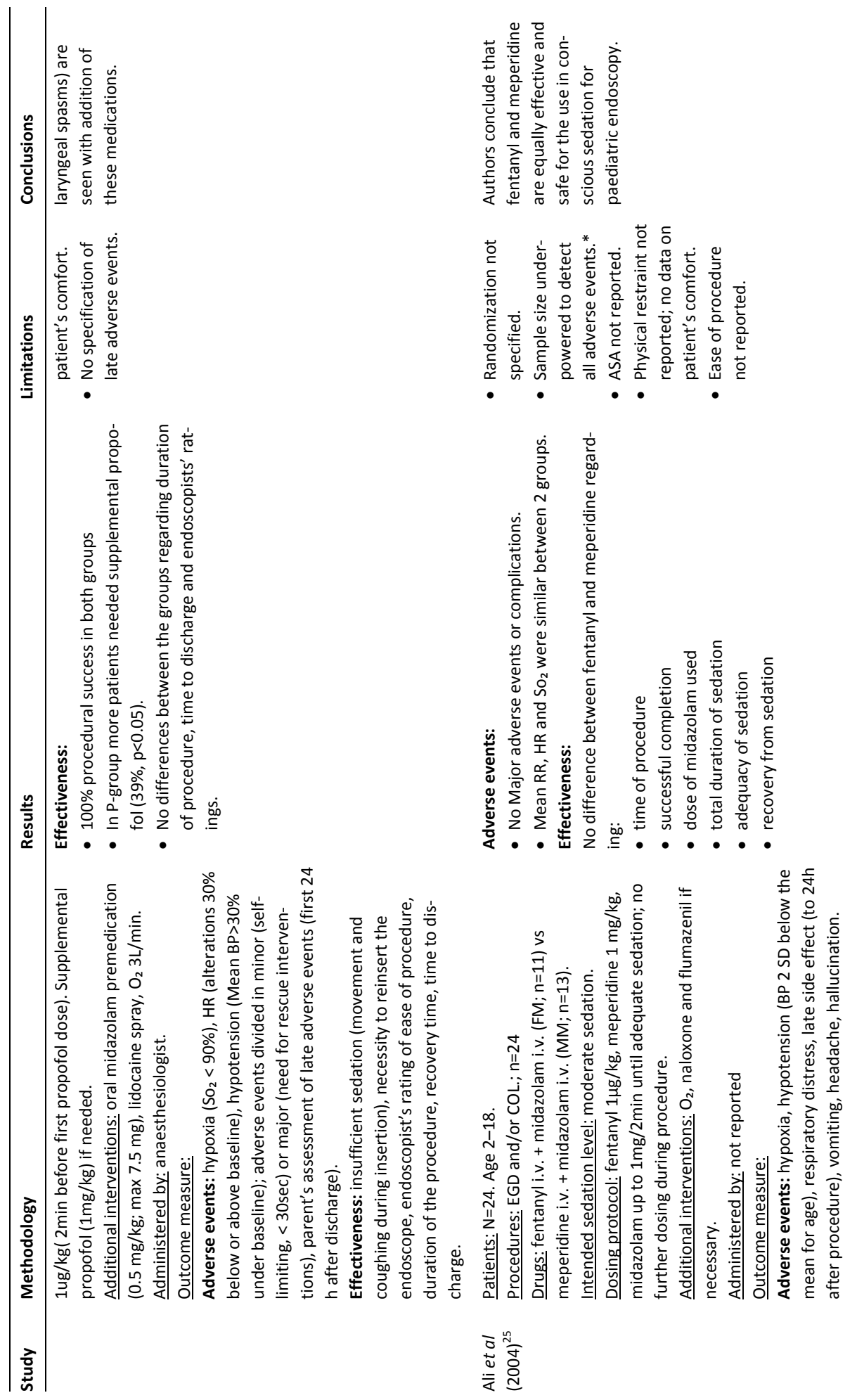




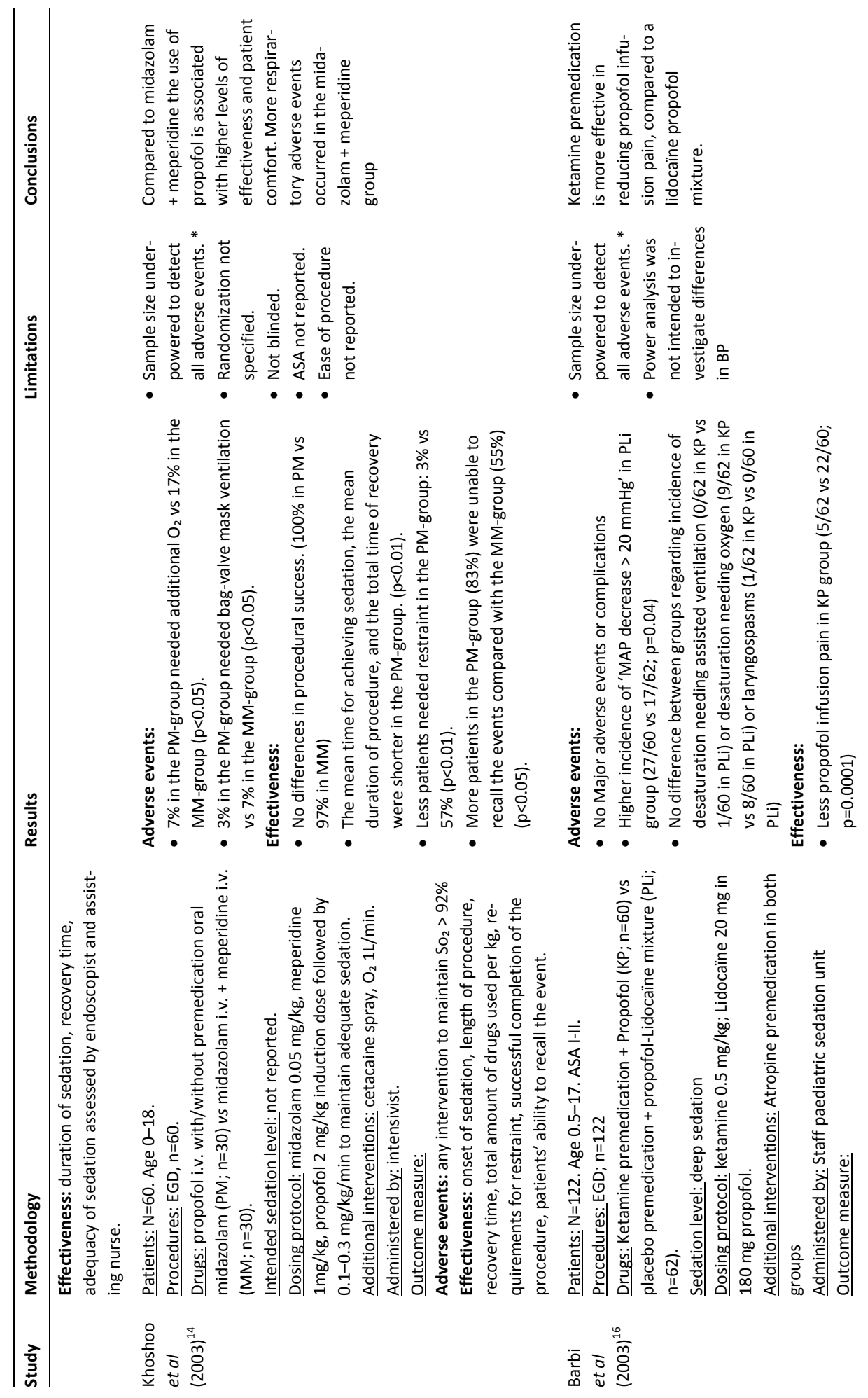




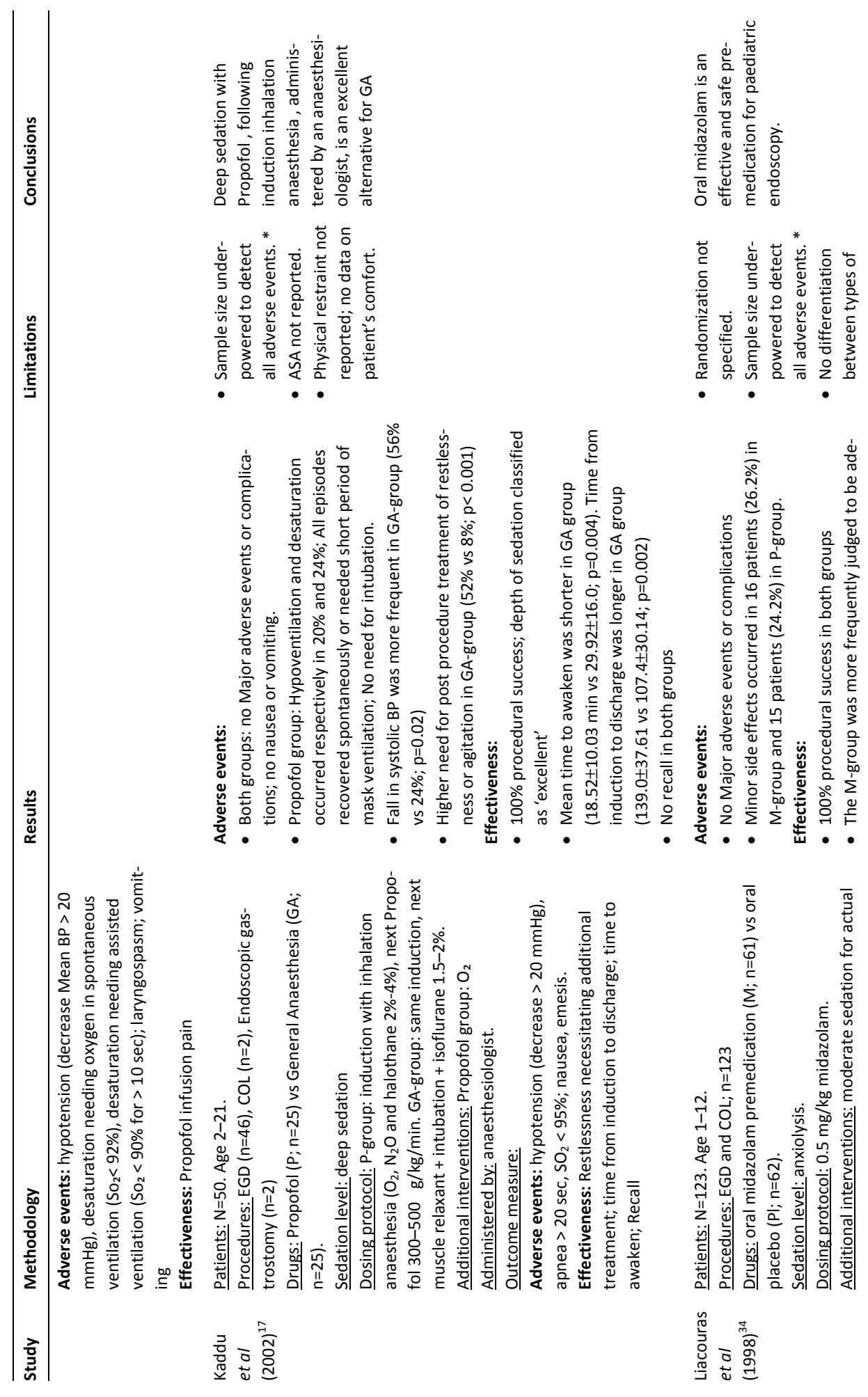




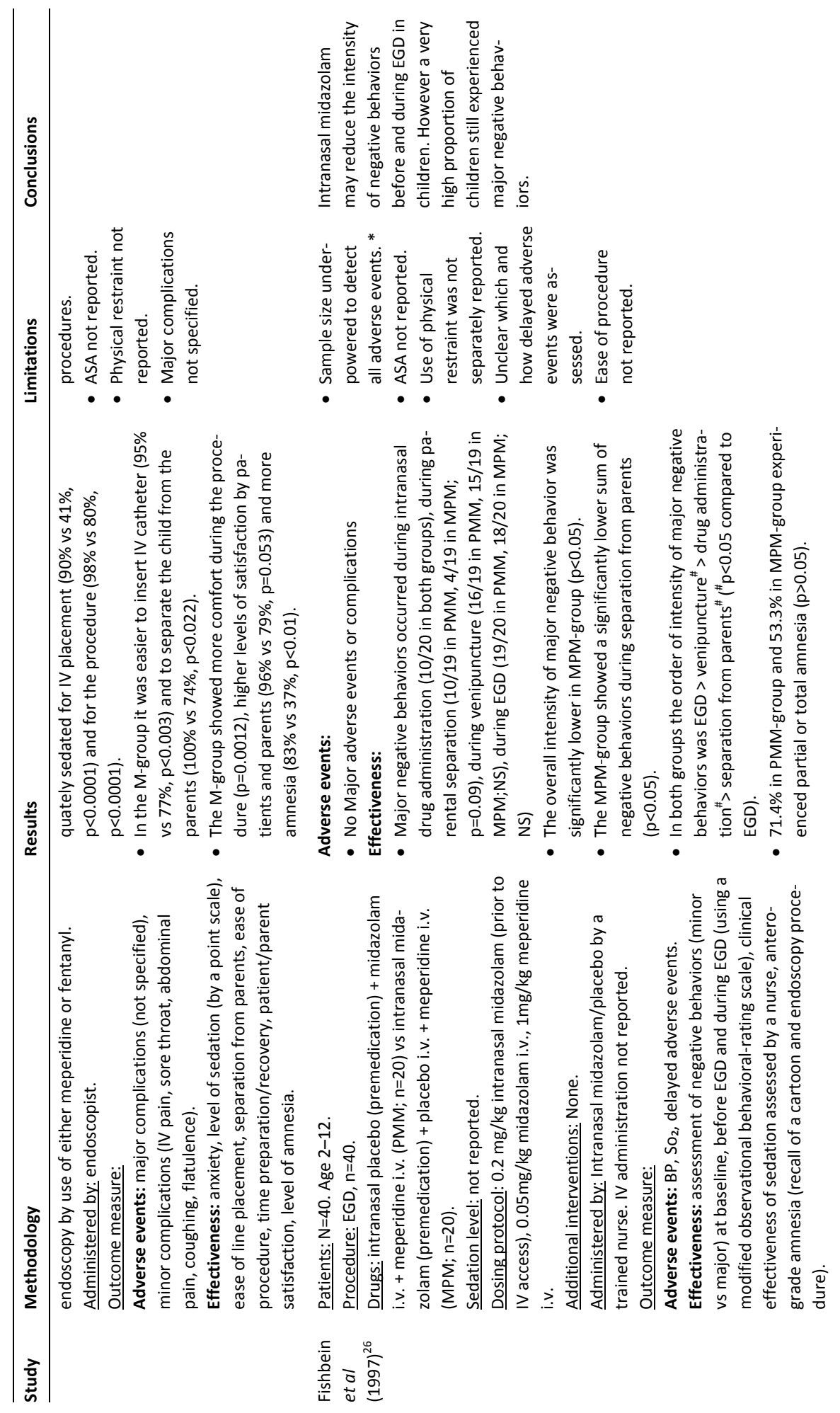




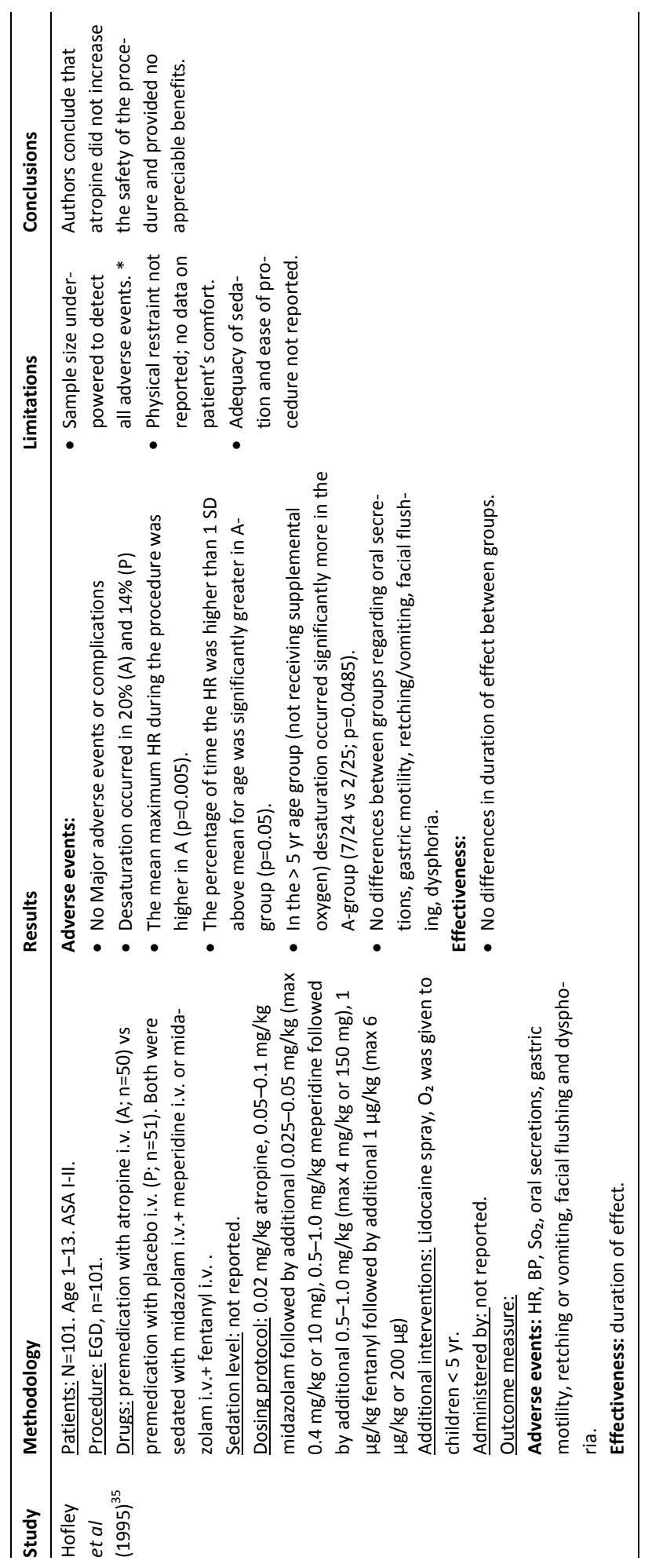




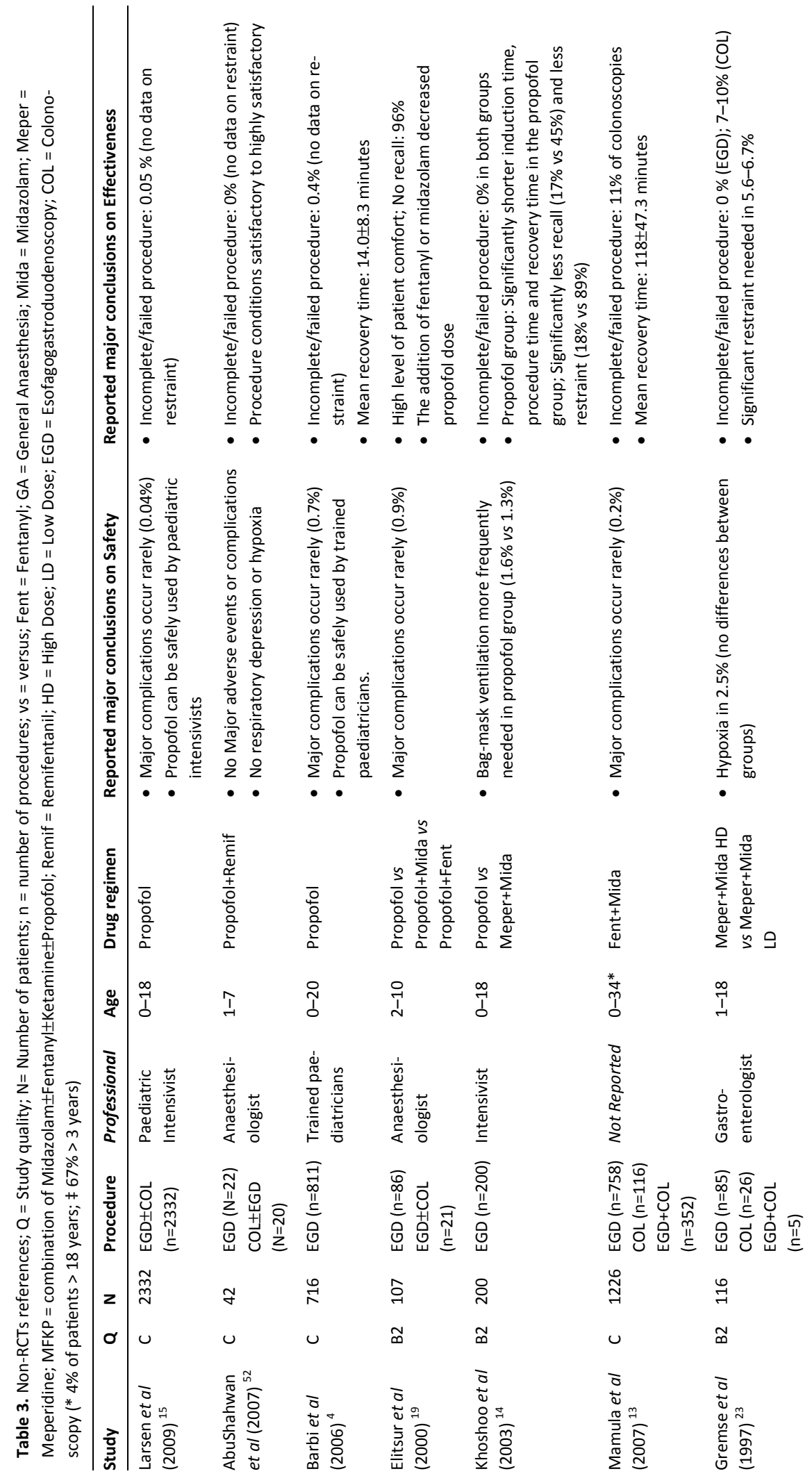




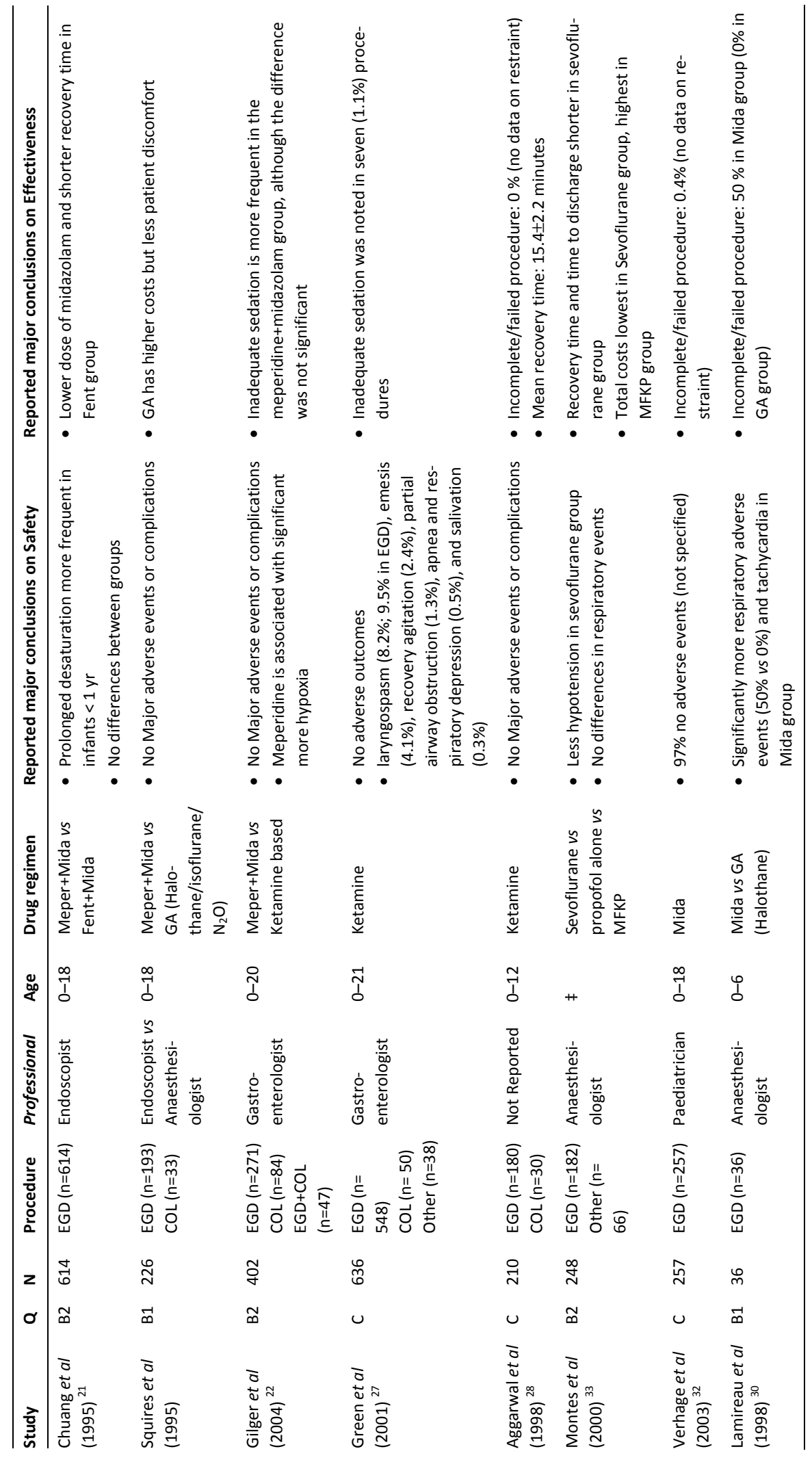




\section{Propofol-based PSA}

Six RCTs ( $N=561$ procedures) and 4 non-RCTs ( $N=3322$ procedures) examined the safety and/or effectiveness of propofol-based PSA. The majority of published propofol sedations (3420/3883; $88.1 \%$ ) were performed by non-anaesthesiologists, i.e. paediatric intensivists ${ }^{14,15}$ or specifically trained paediatricians ${ }^{4,16}$.

Propofol-based PSA is safe. Mild respiratory events occur frequently and major complications may happen rarely, but adverse events do not occur more frequently compared to other sedation regimens. On a total of 3883 reported propofol-based sedations, major respiratory complications like total airway obstruction, deep hypoxia or apnea occurred 11 times (0.3\%). No cases of intubation, resuscitation, permanent sequelae or death were reported. In the largest study (Larsen et al; $\mathrm{N}=2332$ ) the incidence was only $0.04 \%$ but the retrospective design might have caused underreporting. In all studies patients routinely received additional oxygen. Overall, the incidence of adverse events is not higher compared to other sedative regimens included in this systematic review. Minor respiratory events, like temporary desaturation of the arterial blood or hypoventilation occurred more frequently (up to $24 \%$ in Kaddu's study), particularly in infants younger than 1 year old (incidence 35\% compared to $12.5 \%$ in children > 10 years; $\mathrm{p}<0.02) .{ }^{15}$ Three of the six children needing assisted ventilation in the study by Barbi et al $(\mathrm{N}=811$ procedures) were also under 1 year of age. The other three and all 14 children developing a laryngospasm (overall incidence $1.7 \%$ ) were under 4 years of age. ${ }^{4}$ Disma et al reported an incidence of laryngospasm of $3.75 \%$ in children sedated with propofol alone. Adding midazolam or fentanyl to propofol resulted in no cases of laryngospasm. ${ }^{12}$ Clinically relevant hypotension, hypoperfusion or bradycardia have not been reported.

Propofol-based PSA is highly effective. None of the retrieved papers reported in itself on all aspects of effectiveness as defined in the methodology section of this systematic review. The reported incidences of incomplete or failed procedure ranged from $0 \%{ }^{14}$ over $0.05 \%{ }^{15}$ to $0.4 \%{ }^{4}$, ${ }^{15}$. Kaddu et al showed that deep sedation with propofol is an equivalent alternative for general anaesthesia. ${ }^{17}$ The time to achieve adequate sedation, the mean procedure time and the recovery time were significantly shorter in children sedated with propofol, compared to the combined sedation with meperidine plus midazolam. ${ }^{14}$ Only three studies assessed patient comfort or the need for restraint as outcome parameters for effectiveness. Paspatis et al reported an incidence of extreme discomfort of $14.2 \%$ if propofol was used alone. Premedication with oral midazolam improved significantly the level of patient comfort during the procedure $(0 \%$ extreme discomfort). ${ }^{18}$ Khoshoo showed that propofol+midazolam was associated with the need for restraint in only $3 \%$ of cases, compared to $57 \%$ in the meperidine+midazolam group $(P<0.01)$. Total absence of recall was observed more frequently in the propofol+midazolam group ( $88 \%$ versus $55 \%$; $p<$ 0.05). ${ }^{14}$. Elitsur et al reported the absence of pain and recall in $96 \%$ of patients. ${ }^{19}$ Propofol injection pain was reported to occur in $53 \%$ of cases, despite the addition of lidocainne to propofol. ${ }^{4}$

The addition of ketamine, midazolam or fentanyl to propofol-based PSA may have beneficial effects on PSA quality. Tosun et al compared propofol+ketamine with propofol+fentanyl. Both combinations provided equally effective sedation and similar low incidences of mild respiratory events. The use of ketamine was associated with a better tolerance of endoscope insertion but higher incidences of cough, vomiting, dizziness and diplopia. ${ }^{20}$. A low dose of ketamine prior to propofol is more effective in reducing propofol infusion pain, compared to a lidocaïne propofol mixture. ${ }^{16}$ Disma et al showed that the addition of either fentanyl or midazolam caused less adverse events compared to propofol alone. This effect was mainly due to a lower incidence of coughs and laryngeal spasms. No differences were seen between these two additional medi- 
cation regimes regarding effectiveness or adverse events. ${ }^{12}$ Both Disma and Elitsur showed that adding midazolam or fentanyl significantly lowers the necessary dose of propofol. ${ }^{12,19}$

\section{Opioid+benzodiazepine-based PSA}

The effectiveness and/or safety of opioid+benzodiazepine-based sedation were studied in three RCTs ( $N=94$ procedures) and 6 non-RCTs ( $N=2326$ procedures). The administrating professionals were intensivists ${ }^{14}$, gastro-enterologists/endoscopist ${ }^{21-24}$ or were not reported. ${ }^{13,25,26}$

Opioid+benzodiazepine-based sedation is safe. Mild respiratory events occur frequently but major complications may happen occasionally. On a total of 2420 opioid+benzodiazepine-based sedations, major respiratory complications like apnea, total airway obstruction or deep desaturation occurred only 5 times $(0.2 \%)$. In all studies patients received oxygen routinely, except for the studies by Fishbein et al, Chuang et al and Gilger at al.

Koshoo et al showed that the use of meperidine+midazolam was associated with a higher need for supplemental oxygen and ventilatory support, compared to the use of propofol+midazolam (respectively $17 \%$ versus $7 \%$ and $7 \%$ versus $3 \%$; $p<0.05$ for both differences). ${ }^{14}$ Ali et al showed in a small RCT that fentanyl+midazolam and meperidine+midazolam are equally safe. Chuang et al came to the same conclusion in their retrospective comparative study. ${ }^{21}$ Fishbein et al compared intravenous meperidine+midazolam with the combination of intranasal midazolam and intravenous meperidine. Both regimens were equally safe and none of the patients experienced major adverse events. ${ }^{26}$ In their prospective study on intravenous fentanyl+midazolam Mamula et al reported $9 \%$ respiratory adverse events ( $8 \%$ transient desaturation, $1 \%$ prolonged desaturation, $0.2 \%$ apnea), $11 \%$ mild cardiovascular adverse events ( $3 \%$ hypertension, $8 \%$ hypotension), $0.6 \%$ skin rash, $1 \%$ agitation and $5 \%$ vomiting. The following rescue interventions were reported: $9.5 \%$ extra oxygen therapy, $1.3 \%$ tactile stimulation, $0.4 \%$ jaw thrust, $0.2 \%$ bag and mask ventilation and $1.6 \%$ i.v. fluid bolus. In a smaller prospective study Squires et al compared PSA using meperidine+midazolam with general inhalational anaesthesia. No major adverse events or desaturations were reported. ${ }^{24}$ Gilger et al compared retrospectively meperidine+midazolam with meperidine+midazolam+ketamine and midazolam+ketamine. Mild respiratory adverse events, especially desaturations, occurred most frequently in the meperidine+midazolam group $(30.7 \%$ versus respectively $10.9 \%(p<0.001)$ and $0.6 \%(p<0.001) .^{22}$

The evidence indicates that effectiveness of opioid+benzodiazepine based PSA is suboptimal, especially regarding time-characteristics and the need for restraint. None of the retrieved papers reported in itself on all aspects of effectiveness as defined in the methodology section of this systematic review. In their comparative study between propofol+midazolam and meperidine+midazolam Khoshoo reported equally high procedural success rates $( \pm 100 \%)$. However, the time to achieve adequate sedation, the mean procedure time and the recovery time were all significantly longer if midazolam+meperidine was used. Furthermore, restraint was significantly more needed in the meperidine+midazolam group, suggesting a suboptimal effectiveness of this regimen. ${ }^{14}$ Fishbein et al reported very high incidences of major negative behavior during EGD in children sedated with intravenous midazolam+meperidine (19/20) or intranasal midazolam + intravenous meperidine (18/20). The intensity of negative behavior was significantly lower in the group receiving intranasal midazolam premedication. ${ }^{26}$ Ali et al could not show any significant difference in effectiveness between fentanyl-based and meperidine-based PSA, but patient comfort was not an outcome parameter in their study. ${ }^{25}$ In Mamula's study on 
fentanyl+midazolam $11 \%$ of the colonoscopies were incomplete because of inadequate sedation. ${ }^{13}$ Gremse et al found similar figures in their study on meperidine+midazolam. ${ }^{23}$ Gilger et al showed that, compared to ketamine+midazolam, the use of meperidine+midazolam resulted more frequently in inadequate sedation ( $8.6 \%$ versus $3.1 \%)$, although the difference was not significant $(p=0.07) .{ }^{22}$ Finally, a prospective comparison between meperidine+midazolam and general anaesthesia (GA) showed a lower procedural failure rate ( $0 \%$ versus $4.8 \%$ ), lower need for restraint ( $0 \%$ versus $13 \%$ ) and less direct and indirect indications of discomfort in the GA group. $^{24}$

\section{Ketamine-based PSA}

Three non- $\mathrm{RCT}$ trials ( $\mathrm{N}=1056$ procedures) have examined the safety and/or effectiveness of ketamine. In two studies gastroenterologists administered ketamine. ${ }^{22,}{ }^{27}$ Aggarwal did not report the administrating professional. ${ }^{28}$

Ketamine-based PSA is probably safe, but data are limited. Especially in EGD, ketamine is associated with a high chance of laryngeal spasm. Compared to meperidine+midazolam, Gilger et al reported less desaturation in the ketamine+midazolam group. ${ }^{22}$ Aggarwal et al found no particular major adverse events or complications, but it is unclear how vital parameters were assessed. ${ }^{28}$ A retrospective study by Green et al reported more detailed on adverse events. All patients routinely received oxygen therapy. Laryngeal spasm occurred in $9.5 \%$ of EGDs. The only significant independent predictor of laryngospasm in a multivariate analysis was age: $13.9 \%$ in preschool-aged ( $\leq 6$ years) children and $3.6 \%$ in school-aged ( $>6$ years) children (difference $10.3 \%, 95 \% \mathrm{Cl} 5.5-14.9 \%)^{27}$

Ketamine-based PSA is probably effective, but data are limited. Reliable data on patient comfort and the need for restraint are missing. According to Gilger et al inadequate sedation occurrs less frequently in ketamine+midazolam sedation compared to meperidine+midazolam. ${ }^{22}$ Incidences of failed sedation range from $0 \%$ to $1.1 \%$. ${ }^{27,29}$ However, data on patient comfort, the need for restraint or the ease of the procedure are missing.

\section{Midazolam alone}

One RCT ( $\mathrm{N}=61$ procedures) and two non-RCTs ( $\mathrm{N}=275$ procedures) have studied the safety and/or effectiveness of midazolam alone. Either anaesthesiologists ${ }^{30,31}$ or paediatricians ${ }^{32}$ administered midazolam.

Data are too limited to draw conclusions on the safety of midazolam-based PSA. Rafeey et al compared oral and intravenous midazolam. Major adverse events did not occur. The mean oxygen saturation was lower in the intravenous group. ${ }^{31}$ Verhage et al reported an overall incidence of adverse events of $3 \%$. Nature and severity of events were not reported. Flumazenil was needed in $2.7 \%$ of cases. $^{32}$ In a small comparative study Lamireau et al compared intravenous midazolam with halothane anaesthesia. Desaturation below $90 \%$ occurred significantly more frequently in the midazolam group ( $50 \%$ versus $0 \% ; p<0.001) .{ }^{30}$

Midazolam-based PSA is probably ineffective, but data are limited.

Compared to halothane anaesthesia Lamireau et al found that incomplete procedures and complete procedures under difficult conditions occurred significantly more frequently in patients sedated with intravenous midazolam (respectively $50 \%$ versus $0 \%$ and $38.9 \%$ versus $0 \%$ ). ${ }^{30}$ Rafeey and Verhage reported procedural success rates of about $100 \%$. However both studies fail 
to report reliable data on patient comfort, adequacy of sedation and ease of procedure. ${ }^{31,32}$ The significant rise of heart rate and blood pressure during endoscopy, as registered by Rafeey el al, suggests that midazolam is ineffective in reducing patient discomfort.

\section{Sevoflurane inhalation for PSA}

In a retrospective study Montes et al compared inhaled sevoflurane ( $\mathrm{N}=67$ procedures), administered by an anaesthesiologist using an oropharyngeal tube, to two sedative regimens administered by paediatric intensivists: propofol alone ( $N=114$ procedures) and a combination of midazolam and/or fentanyl and/or ketamine and/or propofol ( $\mathrm{N}=67$ procedures). ${ }^{33}$

Sevoflurane-based PSA may be safe, but data are limited? No severe adverse events were reported. Respiratory adverse events occurred rarely at similar incidences in the three groups. The incidence of hypotension was $0 \%$ in de sevoflurane group versus $13.2 \%$ and $7.5 \%$ in the other groups. None of these hypotensive episodes were considered as relevant.

Data are too limited to draw conclusions on the effectiveness of sevoflurane-based PSA. Compared to the intravenous regimens sevoflurane was characterized by a shorter recovery time, earlier discharge and lower costs. This study did not report on patient comfort, need for restraint or overall procedural success. Neither did it specify if and how sevoflurane pollution in the ambient air was avoided. ${ }^{33}$

\section{Premedication}

Three RCTs ( $\mathrm{N}=107$ procedures) specifically analyzed the safety and/or effectiveness of midazolam premedication. One $\mathrm{RCT}$ ( $\mathrm{N}=50$ procedures) analyzed atropine premedication.

Midazolam premedication is probably safe for children undergoing PSA for gastrointestinal endoscopy, but data are limited. None of the studies recorded differences in safety between premedication and non-premedication groups.

Premedication with midazolam has positive effects on patient comfort. There is good evidence that oral midazolam premedication preceding a propofol-based or opioid-based PSA improves significantly the ease and comfort of both IV catheter placement and separating the child from the parents. It also increases the level of patient comfort during the procedure. ${ }^{18,34}$ Following a opioid-based PSA the degree of partial or total amnesia is higher, compared to placebo, if oral midazolam premedication is given (83\% versus $55 \%$; $p<0.05) .{ }^{34}$ In propofol-based PSA the addition of oral midazolam premedication lowers the propofol dose during the procedure but increases the recovery time. ${ }^{18}$ Fishbein et al showed that premedication with intranasal midazolam prior to intravenous meperidine effectively reduces the intensity of negative behaviors. However, no effect was seen on the total number of negative behaviors. Furthermore, the intranasal administration itself was associated with negative behavior in $50 \%$ of cases. ${ }^{26}$

Atropine premedication probably does not increase safety or effectiveness of PSA in children undergoing gastrointestinal endoscopy, but data are limited. Hofley et al compared atropine premedication to placebo in a small group of children sedated with fentanyl+midazolam or meperidine+midazolam. They concluded that atropine did not increase PSA safety and provided no appreciable benefits on oral secretions, gastric motility, vomiting, facial flushing or diphoria. ${ }^{35}$ 


\section{Discussion}

The available evidence suggests that propofol-based PSA is the most effective regimen for PSA during GIE in children. Propofol guarantees an excellent level of procedural success, optimal timing and maximal patient comfort, in particular if propofol is preceded by midazolam premedication. The addition to propofol of intravenous midazolam, fentanyl or remifentanil may increase the effectiveness without generating more adverse events, provided that sedation is performed by trained procedural sedation practitioners under certain quality and safety conditions. Compared to general anaesthesia propofol-based PS seems to be an equally effective technique for selected cases of diagnosis and therapy. It must be noted, however that there is a considerable risk that deep sedation with propofol may result in an actual sedative state close or similar to general anaesthesia for a limited period of time with an increased risk. In a prospective study of children sedated with propofol by non-anaesthesiologists $90 \%$ of children reached a level of "anaesthesia" albeit briefly. ${ }^{36}$ Nevertheless, this systematic review's search category was "sedation" and not "anaesthesia", so that irrespective of the actual sedation level, practitioners thought that they were sedating and not anaesthetizing.

Propofol-infusion-related pain following intravenous injection of propofol can be prevented with a low dose of ketamine, which is in children a more effective approach compared to adding lidocaïne to propofol. Giving a small dose of lidocaïne before injecting propofol could be a very effective alternative but has not been studied in children. Opioid+benzodiazepine-based PSA also results in high procedural success rates. However, the longer time needed to achieve sedation, the longer recovery time and the significantly lower levels of patient comfort are substantial drawbacks compared to propofol-based PSA. There is some evidence that the relatively high incidence of incomplete colonoscopies in opioid+benziodiazepine-based PSA (up to 10\%) is due to ineffective sedation. We could find no study comparing the effectiveness of propofol and ketamine for GIE in children. The available evidence on ketamine suggests less inadequate sedation, compared to meperidine+midazolam. However, none of the papers reported data on patient comfort, the need for restraint or the ease of the procedure. It is plausible that intravenous midazolam alone is not an effective way to provide PSA for GIE in children. However oral midazolam premedication preceding a propofol-based or opioid-based PSA improves significantly the ease and comfort of both IV catheter placement and separating the child from the parents. It also increases the level of patient comfort during the procedure.

Comparing the safety outcome of propofol-based PSA with the more traditional opioid+benziodiazepine-based PSA does not reveal striking differences. Mild and transient hypoxia is reported as the most frequent adverse event in both strategies. Major respiratory adverse events, like deep hypoxia, hypoventilation, apnea and airway obstruction seem to occur rarely but at similar rates. As a consequence both regimens require the same safety precautions. In adult medicine three recent meta-analyses did not show significant differences between propofol-based and traditional PSA for hypoxemia and hypotension, except for fewer cardiorespiratory complications with propofol during colonoscopy. ${ }^{37-39}$ Khoshoo et al published the only available RCT comparing the safety of propofol versus opioid+benzodiazepine-based PSA in children. In the propofol group oxygen therapy and ventilatory support were significantly less needed. There is some evidence that laryngospasm occurs in about $9 \%$ of children sedated with ketamine for $\mathrm{EGD}^{27}$, compared to $1.7-3.7 \%$ if propofol is used alone ${ }^{4,12}$ and $0 \%$ if propofol is combined with midazolam or fentanyl ${ }^{12}$. Therefore it may be prudent to advice not to use ketamine for upper GIE and to add midazolam or fentanyl to a propofol PSA. 
The fact that, apart from one paper ${ }^{4}$, none of the studies made use of capnography monitoring may have caused underestimation of the real incidences of airway obstruction and hypoventilation. The relevance of capnography for early detecting imminent respiratory events during PSA is well established. ${ }^{40-42}$ Its application during GIE has been reviewed recently. ${ }^{43}$

The similar low rate of adverse events in the different sedation regimens is not surprising. It has been shown that PSA related safety is determined by the circumstances, applied safety precautions and professional skills rather than by specific pharmacologic characteristics. ${ }^{10}$ In most studies included in this systematic review, competent and skilled professionals performed PSA in accordance with widely accepted safety guidelines. Adherence to PSA safety guidelines reduces the occurrence of PSA related adverse events. ${ }^{36,44,45}$

The current review suggests the superior effectiveness of propofol, compared to the more traditionally used opioid+benzodiazepine combination. The latter is generally considered as safe in non-anaesthesiologist's hands, while the administration of the anaesthetic propofol is usually restricted for safety reasons to anaesthesiologists only. This distinction is remarkable given the results of this systematic review showing a similar safety profile of both regimens. Furthermore, the vast majority of included propofol sedations were performed by non-anaesthesiologists. Due to limited anaesthesiology resources, propofol is currently being administered worldwide by non-anaesthesiologists (especially trained nurses or endoscopists) for GIE in selected adult patients. ${ }^{46}$ An evidence based guideline on this topic has been published recently. ${ }^{47}$ Also in children, significant evidence exists demonstrating that well-trained non-anaesthesiologists may provide propofol sedation safely, including for gastrointestinal procedures. ${ }^{48}$ It has also been shown that within the setting of adequate training and strict safety measures no differences exists in rates of major complications among different specialist (i.e. anaesthesiologists and nonanaesthesiologists). ${ }^{49}$ Appropriate safety precautions, monitoring and professional skills, rather than professional title, are determinants for the safe and effective use of propofol for PSA. ${ }^{50}$

This study has several limitations. At first, very few randomized controlled trials have compared different pharmacological techniques. The fact that different studies used different outcome measures or different definitions for safety and effectiveness is another important limitation. An objective or validated assessment of the more subjective measures like satisfaction, ease of procedure or patient comfort is missing in most studies. Consequently, it is currently impossible to summarize the available evidence in meta-analyses or to draw solid conclusions on best practice. Nevertheless, we believe that it is possible to draw prudent conclusions and to formulate practical recommendations. Since most of the reviewed studies included both upper and lower GIE we choose not to differentiate between them. It is likely that optimal sedation characteristics and issues on safety and effectiveness are not the same for both procedures. However, we believe that the main conclusions would probably remain the same. Psychological distraction techniques may be useful adjuncts or partial alternatives for PSA. We deliberately choose not to include these non-pharmacological methods in this systematic review. Finally, in individual patients the optimal PSA strategy may deviate from the conclusions of this systematic review. Although the benzodiazepine/opioid combination is not as good as propofol-based PSA, it may be suitable for some children if they can tolerate moderate sedation. Also unsedated endoscopy in selected, highly motivated children has been reported. ${ }^{51}$ In this respect it is remarkable that in Barbi's study among children over the age of 14 years $26.6 \%$ preferred to have no sedation at all during upper GIE. ${ }^{4}$

The limitations of this systematic review clearly identify a gap of scientific knowledge on this topic. Only well-designed, procedure-specific RCT's comparing validated outcome measures on effectiveness and safety between different sedative regimens in large numbers of children may 
yield a definite answer on our clinical question. However, given the results of this systematic review in general and the findings on patient comfort in particular, one may question whether setting up such trials is still ethically justifiable.

\section{Conclusion}

Despite the methodological limitations, the evidence gathered in this systematic review indicates propofol-based PSA as the best practice for PSA in children undergoing GIE. The addition to propofol of intravenous midazolam, fentanyl or remifentanil may increase the safety and effectiveness. Oral midazolam premedication leads to a lower mean dose of propofol, less painful and easier intravenous access, easier separation from the parents and greater patient comfort during endoscopy. A low dose of ketamine is effective in reducing propofol infusion-pain. Propofol is likely to lead to an unconscious state in many children. Whilst this may not be unsafe, it does mean that it is not strictly sedation. Practitioners should be trained to manage this depth of sedation (ie anaesthesia) and parents and children warned that this may happen. Conversely, children who expect to be oblivious to the GIE during sedation with propofol should be told that they may be sedated (not anaesthetized) and that they might recall some details of the procedure. Propofol can be safely administered to children by specifically trained nonanaesthesiologists that provide PSA in adherence to established safety guidelines. In the absence of these professionals and settings it is advisable to refer children that need to undergo a GIE to an anaesthesiologist for propofol-based PSA or anaesthesia. 


\section{Appendix to Part 2.3}

Procedural sedation for gastro-intestinal endoscopy in children. Current practices in Dutch general paediatrics 


\section{Introduction}

Recent literature evidence indicates propofol-based PSA to be the best practice for PSA in children undergoing GIE. This conclusion is in line with the recommendation of the Dutch Society of Paediatrics, stating that children undergoing GIE need anaesthesia or deep sedation. ${ }^{53}$

\section{Methods}

A questionnaire was sent to all paediatricians practicing general paediatric medicine in any of the 97 Dutch hospitals for at least $50 \%$ of their working time in 2008. The list of addressees was based on the member's list of the Dutch Society of Paediatrics and a telephonic enquiry among all paediatric departments. The questionnaire contained questions concerning each paediatrician's PSA practice for GIE examinations in young children (< age of 12 years). No distinction was made between upper and lower GIE.

\section{Results}

At the beginning of 2008,428 specialists were spending at least $50 \%$ of their working time on activities as a general paediatrician. Questionnaires were sent to all these 428 paediatricians. Of the 258 respondents, 67 (25.9\%) had been locally involved in performing a GIE in one of their patients during the previous year. These professionals represented 41 of the 97 paediatric departments (42.3\%). The rest of the respondents used to refer patients for GIE to a specialized endoscopy department in a university hospital. In 20 of the 41 paediatric departments, patients underwent GIE always under general anaesthesia, whereas procedural sedation prescribed by a paediatrician was the standard of care in the remaining 21. 36 of 67 respondents worked in one of these 21 departments. The drugs they use for PSA are summarized in table 4.

Table 4: Overview of medicines that Dutch general paediatricians $(\mathrm{N}=36)$ reported using for PSA during GIE examinations of children.

\begin{tabular}{lc}
\hline Sedative & Frequencies of reported use (\%) \\
\hline Midazolam alone (oral route) & $2(5.5)$ \\
Midazolam alone (rectal route) & $6(16.6)$ \\
Midazolam alone (intravenous route) & $21(58.3)$ \\
Midazolam alone (rout not specified) & $11(30.1)$ \\
Morphine alone (intravenous route) & $3(8.3)$ \\
Fentanyl alone (intravenous route) & $1(2.7)$ \\
Alimemazine & $1(2.7)$ \\
Midazolam+Morpine (both rectal route) & $1(2.7)$ \\
Midazolam+Morphine (intravenous route) & $1(2.7)$ \\
Midazolam+Ketamine (intravenous route) & $1(2.7)$ \\
\hline
\end{tabular}




\section{Discussion}

In about one fifth of the Dutch general paediatric departments, children undergoing GIE receive PSA prescribed by a general paediatrician. Midazolam-alone turned out to be the most popular PSA strategy, whereas benzodiazepine+opioid-based PSA is applied only rarely. Comparing these results with the results of a systematic review of the literature (see chapter 2, part 2.3), we conclude that highly ineffective drugs are commonly used in children undergoing PSA for GIE. Based on data from literature this condition must be associated with a high prevalence in this group of unsuccessful or incomplete procedures. Furthermore, benzodiazepine-alone is ineffective for achieving sufficient patient comfort during GIE and is likely to cause a high need for forced immobilization and restraint.

\section{Conclusion}

Both human considerations regarding patient's right of comfort and good care, as well as scientific evidence indicate that PSA practices for GIE in children must be changed urgently in about one fifth of the Dutch paediatric departments. 


\section{References}

1. Koh JL, Black DD, Leatherman IK, Harrison RD, Schmitz ML. Experience with an anesthesiologist interventional model for endoscopy in a pediatric hospital. Journal of pediatric gastroenterology and nutrition. 2001;33(3):314-318.

2. Leroy PL, Schipper DM, Knape HJ. Professional skills and competence for safe and effective procedural sedation in children: recommendations based on a systematic review of the literature. International journal of pediatrics.2010:934298.

3. Lewis Claar R, Walker LS, Barnard JA. Children's knowledge, anticipatory anxiety, procedural distress, and recall of esophagogastroduodenoscopy. Journal of pediatric gastroenterology and nutrition. 2002;34(1):68-72.

4. Barbi E, Petaros P, Badina L, Pahor T, Giuseppin I, Biasotto E, et al. Deep sedation with propofol for upper gastrointestinal endoscopy in children, administered by specially trained pediatricians: a prospective case series with emphasis on side effects. Endoscopy. 2006;38(4):368-375.

5. Krauss B, Green SM. Procedural sedation and analgesia in children. Lancet. 2006;367(9512):766-780.

6. Babl FE, Oakley E, Seaman C, Barnett P, Sharwood LN. High-concentration nitrous oxide for procedural sedation in children: adverse events and depth of sedation. Pediatrics. 2008;121(3):e528-532.

7. Cote CJ. Round and round we go: sedation -- what is it, who does it, and have we made things safer for children? Paediatric anaesthesia. 2008;18(1):3-8.

8. Sury MR, Smith JH. Deep sedation and minimal anesthesia. Paediatric anaesthesia. 2008;18(1):18-24.

9. Vangerven M, Van Hemelrijck J, Wouters $P$, Vandermeersch E, Van Aken H. Light anaesthesia with propofol for paediatric MRI. Anaesthesia. 1992;47(8):706-707.

10. Cote CJ, Notterman DA, Karl HW, Weinberg JA, McCloskey C. Adverse sedation events in pediatrics: a critical incident analysis of contributing factors. Pediatrics. 2000;105(4 Pt 1):805-814.

11. Lightdale JR, Mahoney LB, Schwarz SM, Liacouras CA. Methods of sedation in pediatric endoscopy: a survey of NASPGHAN members. J Pediatr Gastroenterol Nutr. 2007;45(4):500-502.

12. Disma N, Astuto M, Rizzo G, Rosano G, Naso P, Aprile G, et al. Propofol sedation with fentanyl or midazolam during oesophagogastroduodenoscopy in children. European journal of anaesthesiology. 2005;22(11):848-852.

13. Mamula P, Markowitz JE, Neiswender K, Zimmerman A, Wood S, Garofolo M, et al. Safety of intravenous midazolam and fentanyl for pediatric GI endoscopy: prospective study of 1578 endoscopies. Gastrointestinal endoscopy. 2007;65(2):203-210.

14. Khoshoo V, Thoppil D, Landry L, Brown S, Ross G. Propofol versus midazolam plus meperidine for sedation during ambulatory esophagogastroduodenoscopy. Journal of pediatric gastroenterology and nutrition. 2003;37(2):146-149.

15. Larsen R, Galloway D, Wadera S, Kjar D, Hardy D, Mirkes C, et al. Safety of propofol sedation for pediatric outpatient procedures. Clinical pediatrics. 2009;48(8):819-823.

16. Barbi E, Marchetti F, Gerarduzzi T, Neri E, Gagliardo A, Sarti A, et al. Pretreatment with intravenous ketamine reduces propofol injection pain. Paediatric anaesthesia. 2003;13(9):764-768.

17. Kaddu R, Bhattacharya D, Metriyakool K, Thomas R, Tolia V. Propofol compared with general anesthesia for pediatric GI endoscopy: is propofol better? Gastrointestinal endoscopy. 2002;55(1):27-32.

18. Paspatis GA, Charoniti I, Manolaraki M, Vardas E, Papanikolaou N, Anastasiadou A, et al. Synergistic sedation with oral midazolam as a premedication and intravenous propofol versus intravenous propofol alone in upper gastrointestinal endoscopies in children: a prospective, randomized study. Journal of pediatric gastroenterology and nutrition. 2006;43(2):195-199.

19. Elitsur Y, Blankenship P,Lawrence Z. Propofol sedation for endoscopic procedures in children. Endoscopy. 2000;32(10):788-791.

20. Tosun Z, Aksu R, Guler G, Esmaoglu A, Akin A, Aslan D, et al. Propofol-ketamine vs propofol-fentanyl for sedation during pediatric upper gastrointestinal endoscopy. Paediatric anaesthesia. 2007;17(10):983988.

21. Chuang E, Wenner WJ, Jr., Piccoli DA, Altschuler SM, Liacouras CA. Intravenous sedation in pediatric upper gastrointestinal endoscopy. Gastrointestinal endoscopy. 1995;42(2):156-160. 
22. Gilger MA, Spearman RS, Dietrich CL, Spearman G, Wilsey MJ, Jr., Zayat MN. Safety and effectiveness of ketamine as a sedative agent for pediatric GI endoscopy. Gastrointestinal endoscopy. 2004;59(6):659663.

23. Gremse DA, Kumar S, Sacks Al. Conscious sedation with high-dose midazolam for pediatric gastrointestinal endoscopy. Southern medical journal. 1997;90(8):821-825.

24. Squires RH, Jr., Morriss F, Schluterman S, Drews B, Galyen L, Brown KO. Efficacy, safety, and cost of intravenous sedation versus general anesthesia in children undergoing endoscopic procedures. Gastrointestinal endoscopy. 1995;41(2):99-104.

25. Ali S, Davidson DL, Gremse DA. Comparison of fentanyl versus meperidine for analgesia in pediatric gastrointestinal endoscopy. Digestive diseases and sciences. 2004;49(5):888-891.

26. Fishbein M, Lugo RA, Woodland J, Lininger B, Linscheid T. Evaluation of intranasal midazolam in children undergoing esophagogastroduodenoscopy. Journal of pediatric gastroenterology and nutrition. 1997;25(3):261-266.

27. Green SM, Klooster M, Harris T, Lynch EL, Rothrock SG. Ketamine sedation for pediatric gastroenterology procedures. Journal of pediatric gastroenterology and nutrition. 2001;32(1):26-33.

28. Aggarwal A, Ganguly S, Anand VK, Patwari AK. Efficacy and safety of intravenous ketamine for sedation and analgesia during pediatric endoscopic procedures. Indian pediatrics. 1998;35(12):1211-1214.

29. Agrawal D, Manzi SF, Gupta R, Krauss B. Preprocedural fasting state and adverse events in children undergoing procedural sedation and analgesia in a pediatric emergency department. Annals of emergency medicine. 2003;42(5):636-646.

30. Lamireau T, Dubreuil M, Daconceicao M. Oxygen saturation during esophagogastroduodenoscopy in children: general anesthesia versus intravenous sedation. Journal of pediatric gastroenterology and nutrition. 1998;27(2):172-175.

31. Rafeey M, Ghojazadeh M, Feizo Allah Zadeh H, Majidi H. Use of oral midazolam in pediatric upper gastrointestinal endoscopy. Pediatr Int.52(2):191-195.

32. Verhage J, Mulder CJ, Willekens FL. Intravenous midazolam sedation in pediatric diagnostic upper digestive endoscopy. A prospective study in a general hospital. Romanian journal of gastroenterology. 2003;12(4):273-276.

33. Montes RG, Bohn RA. Deep sedation with inhaled sevoflurane for pediatric outpatient gastrointestinal endoscopy. Journal of pediatric gastroenterology and nutrition. 2000;31(1):41-46.

34. Liacouras CA, Mascarenhas M, Poon C, Wenner WJ. Placebo-controlled trial assessing the use of oral midazolam as a premedication to conscious sedation for pediatric endoscopy. Gastrointestinal endoscopy. 1998;47(6):455-460.

35. Hofley MA, Hofley PM, Keon TP, Gallagher PR, Poon C, Liacouras CA. A placebo-controlled trial using intravenous atropine as an adjunct to conscious sedation in pediatric esophagogastroduodenoscopy. Gastrointestinal endoscopy. 1995;42(5):457-460.

36. Barbi E, Gerarduzzi T, Marchetti F, Neri E, Verucci E, Bruno I, et al. Deep sedation with propofol by nonanesthesiologists: a prospective pediatric experience. Archives of pediatrics \& adolescent medicine. 2003;157(11):1097-1103.

37. McQuaid KR, Laine L. A systematic review and meta-analysis of randomized, controlled trials of moderate sedation for routine endoscopic procedures. Gastrointestinal endoscopy. 2008;67(6):910-923.

38. Qadeer MA, Vargo JJ, Khandwala F, Lopez R, Zuccaro G. Propofol versus traditional sedative agents for gastrointestinal endoscopy: a meta-analysis. Clin Gastroenterol Hepatol. 2005;3(11):1049-1056.

39. Singh H, Poluha W, Cheung M, Choptain N, Baron KI, Taback SP. Propofol for sedation during colonoscopy. Cochrane database of systematic reviews (Online). 2008(4):CD006268.

40. Cote $\mathrm{CJ}$, Wilson S. Guidelines for monitoring and management of pediatric patients during and after sedation for diagnostic and therapeutic procedures: an update. Pediatrics. 2006;118(6):2587-2602.

41. Krauss B, Hess DR. Capnography for procedural sedation and analgesia in the emergency department. Annals of emergency medicine. 2007;50(2):172-181.

42. Yarchi D, Cohen A, Umansky T, Sukhotnik I, Shaoul R. Assessment of end-tidal carbon dioxide during pediatric and adult sedation for endoscopic procedures. Gastrointestinal endoscopy. 2009;69(4):877882 . 
43. Gerstenberger PD. Capnography and patient safety for endoscopy. Clin Gastroenterol Hepatol.8(5):423425.

44. Hoffman GM, Nowakowski R, Troshynski TJ, Berens RJ, Weisman SJ. Risk reduction in pediatric procedural sedation by application of an American Academy of Pediatrics/American Society of Anesthesiologists process model. Pediatrics. 2002;109(2):236-243.

45. Pitetti R, Davis PJ, Redlinger R, White J, Wiener E, Calhoun KH. Effect on hospital-wide sedation practices after implementation of the 2001 JCAHO procedural sedation and analgesia guidelines. Archives of pediatrics \& adolescent medicine. 2006;160(2):211-216.

46. Rex DK, Deenadayalu VP, Eid E, Imperiale TF, Walker JA, Sandhu K, et al. Endoscopist-directed administration of propofol: a worldwide safety experience. Gastroenterology. 2009;137(4):1229-1237; quiz 1518-1229.

47. Dumonceau JM, Riphaus A, Aparicio JR, Beilenhoff U, Knape JT, Ortmann M, et al. European Society of Gastrointestinal Endoscopy, European Society of Gastroenterology and Endoscopy Nurses and Associates, and the European Society of Anaesthesiology Guideline: Non-anesthesiologist administration of propofol for GI endoscopy. Endoscopy.42(11):960-974.

48. Cravero JP, Beach ML, Blike GT, Gallagher SM, Hertzog JH. The incidence and nature of adverse events during pediatric sedation/anesthesia with propofol for procedures outside the operating room: a report from the Pediatric Sedation Research Consortium. Anesthesia and analgesia. 2009;108(3):795-804.

49. Couloures KG, Beach M, Cravero JP, Monroe KK, Hertzog JH. Impact of provider specialty on pediatric procedural sedation complication rates. Pediatrics.127(5):e1154-1160.

50. Green SM, Krauss B. Barriers to propofol use in emergency medicine. Annals of emergency medicine. 2008;52(4):392-398.

51. Bishop PR, Nowicki MJ, May WL, Elkin D, Parker PH. Unsedated upper endoscopy in children. Gastrointestinal endoscopy. 2002;55(6):624-630.

52. Abu-Shahwan I, Mack D. Propofol and remifentanil for deep sedation in children undergoing gastrointestinal endoscopy. Paediatric anaesthesia. 2007;17(5):460-463.

53. NVK Richtlijn Inflammatoire darmziekten bij kinderen (IBD) $2008 \mathrm{http}: / /$ www.nvk.nl/Kwaliteit/Richtlijnenenindicatoren/Richtlijnen/IBD/tabid/332/language/nl-NL/Default.aspx. 
Part 2.4 Providing procedural sedation/analgesia in children. How satisfied are Dutch general paediatricians with their personal performance?

Piet LJM Leroy, Fred HM Nieman, Heleen E Blokland-Loggers, Luc JI Zimmermann.

Submitted 


\begin{abstract}
Objectives: Procedural sedation/analgesia (PSA) in Dutch hospitalized children is usually provided by non-trained general paediatricians. However, these professionals mostly fail to adhere to established safety guidelines. Before setting up a strategy for improvement we wanted to know how (dis)satisfied general paediatricians are with their personal way of providing procedural sedation. A more profound understanding of the reasons for (dis)satisfaction may lead to possible handles for the enhancement of guideline adherence. We hypothesize that personal beliefs and individual background characteristics predict the level of (dis)satisfaction with personal performance.
\end{abstract}

Study design and methods: A nationwide survey was conducted amongst general paediatricians. Selected Personal beliefs were operationalized into Likert-scale items with which respondents could agree or disagree. Factor and reliability analysis on item scores resulted in 10 psychometric scales with a high internal consistency. The hypothesis was tested by multiple regression analysis.

Results: (Dis)satisfaction with own practice showed large variations amongst respondents. Beliefs of 'relevance as a problem', 'extra burden', 'worrying about safety', 'the lack of support by anaesthesiologists' in less confident practitioners and 'the lack of appropriate material and infrastructure' in protocol users were significant predictors of dissatisfaction with own practice (78.2\% explained variance).

Conclusions: Explaining the variance of (dis)satisfaction with personal performance reveals interesting handles for the enhancement of the level of adherence to new guidelines. 


\section{Introduction}

Invasive diagnostic and therapeutic procedures are part of daily paediatric practice. Many of these procedures are painful, stressful or impossible to perform without immobilizing the patient. Therefore children may need procedural sedation and/or analgesia (PSA). PSA can be defined as the use of sedative, analgesic, or dissociative drugs to provide anxiolysis, analgesia, sedation and motor control during painful or unpleasant procedures. ${ }^{1}$ Due to limited anaesthesia services PSA is often provided by non-anaesthesiologists, who usually have not received a formal training in it. ${ }^{2}$ Following the report of severe PSA related accidents in children, concerns about the safety of this practice led to the publication of safety guidelines on PSA. ${ }^{1,3-8}$ All these guidelines specify similar safety precautions including the assessment of the sedation risk prior to PSA and guidelines on a proper fasting status, appropriate monitoring, recovery standards, professional competence, and imperative rescue facilities. It has been shown that adherence to these guidelines will lead to safer PSA in children. ${ }^{9-11}$

In the Netherlands PSA for common procedures (e.g. diagnostic imaging) in hospitalized children is mostly prescribed by general paediatricians. A national guideline on PSA was published in 1998 and has been adopted by the Dutch Society of Paediatrics as their official guideline on this topic. Despite the availability of this guideline PSA is still associated with important problems in Dutch paediatrics. A recent quality audit by the Dutch Society of Paediatrics revealed that general paediatricians report sedation failure, unsafety, lack of competence and especially a lack of anaesthetic support as highly relevant problems in their daily PSA practice. In the last decade at least 3 severe accidents ( 2 with a fatal outcome and 1 with permanent damage) have happened in children during PSA. In a nationwide survey of general paediatricians we recently have shown that the level of adherence to established safety guidelines is rather low. ${ }^{12}$ Therefore, (regularly repeated) interventions are urgently needed to enhance guideline adherence. Measuring the level of actual adherence to guidelines may reveal to what extent it should be enhanced. However, it does not simply indicate which interventions may be needed to boost individual adherence to a level that guarantees a close to optimal safety. Before setting up a strategy for guideline implementation a deeper understanding of the individual variations and characteristics of the target population (i.e. PSA providers) is advisable.

In order to understand fundamentally the issue of practicing with low adherence, it must be looked upon as a habit-like behaviour. Habit-like behaviour is not an isolated phenomenon but rather the result of latent and progressively developing subjective beliefs emerging from the interaction between (external) norms and own actions. As far as 'adherence to a guideline' is concerned, an important general belief is the extent in which paediatricians are satisfied or dissatisfied with their own PSA performance seen in the light of fulfilment to the professional norms they have to adhere to. Measuring satisfaction and dissatisfaction is important: if professionals are dissatisfied with their performance, it surely will reflect itself in their actual adherence behaviour. There is good evidence from literature that feelings of frustration, dissatisfaction with personal practice or general unwellness have negative effects on workplace productivity and efficiency but also on quality of patient care and patient safety. ${ }^{13}$

The purpose of this study was to operationalize, measure and analyze (dis)satisfaction with personal PSA practice in Dutch general pediatricians entrusted with PSA. We hypothesize that satisfaction or dissatisfaction with self-performed PSA can be explained by specific underlying motivational beliefs professionals have (developed) on PSA and by individual background variables they can be characterized with. (Figure 1) We believe that analyzing the assumed relation- 
ships will reveal interesting viewpoints on both the way paediatricians in general view and experience their personal PSA practice and on the type of problems they generally say to encounter. By a more profound understanding of the sources and variations of their (dis)satisfaction we aim to define more practical advice for the improvement of adherence to PSA guidelines among Dutch general paediatricians.

\section{PREDICTORS OUTCOME}

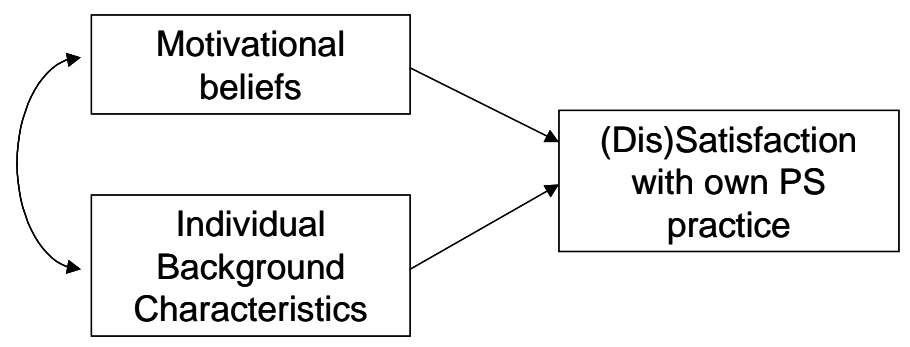

Figure 1: Hypothesis of this study

\section{Materials \& Methods}

\section{Study population}

The elective population for the survey is defined as: all paediatricians who during 2007 were clinically active in general paediatrics for at least $50 \%$ of their working time within one of the 97 Dutch hospitals ánd who had themselves performed at least one PSA procedure during the 12 months preceding this study. Names, addresses and hospitals of the elective paediatricians were collected by use of the records of the Dutch Society of Paediatrics and by a telephonic inquiry of all Dutch paediatric departments. For the study it was decided to contact $100 \%$ of the elective population.

\section{Method of observation}

Two self-administered postal questionnaires delivered within an interval of 3 months, were used. The first one included questions on the background characteristics of the respondent and a procedural practice list on which respondent could score what type of, how and how many times parts of the PSA were actually on average performed during the preceding 12 months. The second questionnaire concentrated upon psychometric measurements comprising motivational beliefs and feelings on practicing PSA and the central concept of satisfaction or dissatisfaction with own performance of PSA. Both questionnaires were followed by one prompting/reminder 2 months later. 


\section{Measuring respondent's background characteristics}

Individual background characteristics were measured via direct questions on age, gender, number of years of working experience, personal use of any PSA protocol and contextual background factors of the hospital unit, where the respondent is actually working ('teaching qualification', 'number of beds', 'number of medical staff personnel' and the 'existence of and/or (active) participation in a local PSA protocol group').

\section{Measuring (dis)satisfaction with and underlying motivational beliefs on performing PSA}

Motivational types of beliefs on performing PSA were found and selected by interviewing a small group of general paediatricians on their personal concerns about performing PSA. This resulted in 12 domains, one for (dis)satisfaction and 11 for possible underlying motivational beliefs. (Appendix $A)$

Overall (dis)satisfaction with performing PSA was operationalized in two-ways, i.e. firstly as (dis)satisfaction with the way the PSA procedure had been implemented at the department where the respondent was professionally active and secondly as (dis)satisfaction with the way the respondent him/herself had been planning, arranging and performing PSA during the past 12 months. For both subconcepts positive and negative verbal statements (items) were gathered expressing respectively satisfaction and dissatisfaction with the general daily PSA practice. The same procedure of operationalization was used for all defined motivational beliefs (perceptions, expectations, and other types of evaluations). Because no validated questionnaire from literature was available for this purpose, a new one had to be constructed. Statements to be used in items were judged on their face and content validity only. In their initial state, they were presented as a pre-testing list to a similar group of the intended practitioners, who were not included in the elective population of the survey. After useful suggestions to improve the existing list (expert validity), a revised and shortened list of statements was used in the eventual questionnaire. Finally, a total of 78 statements were presented in a final list as 5-point items (Likert scales) on which respondents could either agree or very much agree, or could disagree or very much disagree. The midpoint of the scale was reserved for the position: "I equally agree, as well as equally disagree". An outer-scale, sixth position could either be used for respondents who felt that they had no opinion whatsoever on the statement in the item, or for respondents who felt that the case or the description of it mentioned within the item did not apply to their working situation. Because the questionnaire list was brand-new, and extended psychometric testing after measurement often implies dumping of mal-phrased, non-valid and unreliable items, the total number of positively and negatively stated items was initially set at about six per study domain. Only for the central concept in the study, overall (dis)satisfaction with own practice, an exception to this rule was made: to enhance its reliability 10 items were initially used to operationalize it (i.e. 5 for each subconcept). For each of the 12 domains one eventual onedimensional scale was planned to be measured by all items belonging to it.

\section{Testing the hypothesis of this study}

Univariate and multivariate statistics were used to examine the relationships between respondent's level of (dis)satisfaction on the one hand and motivational beliefs and/or individual background characteristics on the other hand. 


\section{Statistics}

\section{a. Scale construction}

If metric data were normally distributed (tested by Kolmogorow-Smirnow (K-S)) means and standard deviations were calculated. For non-normally distributed metric data medians and score ranges were also given. Categorical data were presented by frequencies and percentages. Ordinal-scale five-point Likert-scales used in items for beliefs were assumed to have equal distances between scores; so metric characteristics can more suitably be applied. Relationships between metric data were measured by Pearson product-moment correlation coefficients ( $r$ ). Multiple statistical relationships between item-scores in and between domains presumed to be conceptually related to each other were analyzed by principal components analysis (PCA) using pairwise and listwise deletion of missing cases. Initial matrices of component loadings were transformed to pattern matrices of factor loadings using oblique rotation. In solutions a Thurstone Simple Structure was aimed at, where items had high loadings on only one factorial dimension (component) and (ideally) very low loadings on other dimensions. Bi- or multidimensionally loading items were removed from successive renewed runs of PCA without such items. Factor/component loadings in the final solution had to be less than -0.50 or higher than +0.50 . Next, internal component stability was measured by calculating Cronbach's alpha for each component separately. Alpha had to be higher than 0.70 for each component and a specific item was removed, if alpha for scale minus this item was heightened, which procedure was again repeated for remaining items. Next, scale constructions were additively computed using the average score over items validly scored. If scores of half of the number of items plus 1 belonging to a factor were non-missing, average scale scores were computed, if not, scale scores were set as missing.

\section{b. Multiple regression analysis}

Multiple regression analysis was performed with overall (dis)satisfaction with own practice in PSA as a 'dependent' variable and all other psycho-social scales plus background characteristics as predictors using listwise deletion of missing cases. Forward selection as well as backward elimination techniques of entering the regression equation were used. A cross-section of predictors that had shown statistical significance in both forward and backward procedure was used to calculate effects within a pre-final forced-entry regression model. Finally, a final model was found after testing all first-order interactions of all possible pairs of remaining predictors. A $p$ value of less than 0.05 was used to define statistical significance. All data analysis was done with SPSS-pc, version 16.0.

\section{Results}

\section{Response rate}

By the end of 2007428 specialists were clinically active in general paediatrics for at least $50 \%$ in one of the 97 hospitals in the Netherlands. Since PSA is not a formally registered activity in The Netherlands it was impossible to define the elective population precisely in advance. Therefore questionnaires were sent to all 428 specialists. Questionnaire one and two were returned by respectively 258 and 223 of them. Respectively 197 of the 258 respondents on questionnaire 
one $(76.4 \%)$ and 175 of the 223 on questionnaire two (78.5\%) reported to have performed PSA personally during the preceding 12 months. Given the fact that about $77 \%$ of respondents turned out to have personal experience with PSA, we estimate that the elective population amounts to about 330 specialists (i.e. 77\% of 428). Response rates can therefore be estimated to be about $59.7 \%$ (questionnaire one) and $53.0 \%$ (questionnaire two). In order to analyze motivational beliefs on practicing PSA, as well as individual background characteristics as possible predictors of (dis)satisfaction the databases of both parts of the questionnaire were combined in one data file. This resulted in an extra loss of 20 of the 175 respondents (11.4\%). The combined responses ( $\mathrm{N}=155 ; 46.9 \%$ of the elective population) were sufficiently complete for data analysis. Remaining respondents included general paediatricians working in 88 of the $97(90.7 \%)$ hospitals.

\section{Background Characteristics $(N=197)$}

101 respondents (51.3\%) were male. Overall mean age was 46.7 years (SD 7.8;range 31-63). The median reported numbers of years of experience was 11 years (range 1-31). Demographics and regional distribution of respondents were similar to those of the total population of Dutch general paediatricians (data not shown). Respectively 89 (45.2\%), 76 (38.6\%) and $32(16.2 \%)$ respondents reported to have performed PSA personally less than 1 time a month, 1 to 4 times a month and more than 4 times a month. Procedures for which respondents reported to use PSA were diagnostic imaging $(81.7 \%)$, wound care $(33.5 \%)$, endoscopy $(27.9 \%)$ and lumbar puncture (27.9\%). Out of 195 respondents 59 (29.9\%) reported never to use a protocol for PSA. 81 (41.1\%) of the respondents reported the existence of a local working group on paediatric PSA in which 23 respondents $(11.7 \%)$ were personally and actively involved.

\section{Analyzing (dis)satisfaction}

Results of Psychometric scale construction ( $N=175)$

Principle component and reliability analysis of the 12 domains resulted in only a slight rearrangement of some items in 10 definitive psychometric scales. A high internal consistency was attained. The two subconcepts of overall (dis)satisfaction had so high internal correlations that only one scale remained for the outcome variable. Furthermore, 9 scales for motivational beliefs (i.e. potential predictors of the outcome variable) remained after psychometric analysis. The 10 observed scales included 69 out of the initially used 78 items. Definitions and descriptive statistics of the definitive scales are summarized in table 1 . Only a minority of this population showed high levels of satisfaction with personal PSA practice. High level of agreement with positive beliefs (e.g. feeling of positive support by anaesthesiologists) and high level of disagreement with negative beliefs (e.g. concerns on risking failure, worrying about safety, perception of lack of appropriate materials and infrastructure) turned out to occur rarely. 
Table 1: Descriptive statistics and Cronbach alpha's $(C \alpha)$ of definitive psychometric scales constructed after principal component and reliability analysis (see methods section). ( $N=175)$

\begin{tabular}{|c|c|c|c|c|c|c|c|c|c|}
\hline \multicolumn{5}{|c|}{ Scale } & \multicolumn{5}{|c|}{ Descriptive Statistics } \\
\hline Description & $\begin{array}{l}\text { Number } \\
\text { of Items }\end{array}$ & Range & Value labels & $\mathrm{C} \alpha$ & $\mathrm{N}$ & Min & Max & Mean & SD \\
\hline $\begin{array}{l}\text { (Dis)satisfaction regarding } \\
\text { the state of affairs of self- } \\
\text { performed PSA }\end{array}$ & 9 & $1-5$ & $\begin{array}{l}\text { 1= Highly satisfied } \\
5=\text { Highly dissatis- } \\
\text { fied }\end{array}$ & 0.89 & 166 & 1.11 & 4.78 & 3.01 & 0.64 \\
\hline $\begin{array}{l}\text { Self-evaluated lack of skills } \\
\text { and knowledge to perform } \\
\text { PSA properly }\end{array}$ & 12 & $1-5$ & $\begin{array}{l}1=\text { Highly dis- } \\
\text { agree } \\
5=\text { Highly agree }\end{array}$ & 0.90 & 172 & 1.38 & 4.63 & 2.86 & 0.67 \\
\hline $\begin{array}{l}\text { Evaluation of positive sup- } \\
\text { port by anaesthesiologists in } \\
\text { solving PSA related issues }\end{array}$ & 4 & $1-5$ & $\begin{array}{l}1=\text { Highly agree } \\
5=\text { Highly dis- } \\
\text { agree }\end{array}$ & 0.92 & 172 & 1.00 & 5.00 & 3.35 & 1.02 \\
\hline $\begin{array}{l}\text { Evaluation of positive sup- } \\
\text { port by diagnostic personnel } \\
\text { in handling PSA during diag- } \\
\text { nostic procedures }\end{array}$ & 4 & $1-5$ & $\begin{array}{l}1=\text { Highly agree } \\
5=\text { Highly dis- } \\
\text { agree }\end{array}$ & 0.90 & 169 & 1.00 & 4.75 & 2.85 & 0.76 \\
\hline $\begin{array}{l}\text { Concern on risking failure of } \\
\text { self-performed PSA }\end{array}$ & 12 & $1-5$ & $\begin{array}{l}\text { 1= Highly dis- } \\
\text { agree } \\
5=\text { Highly agree }\end{array}$ & 0.92 & 169 & 1.10 & 4.90 & 3.04 & 0.70 \\
\hline $\begin{array}{l}\text { Evaluation of importance and } \\
\text { relevance of PSA as a prob- } \\
\text { lem }\end{array}$ & 5 & $1-5$ & $\begin{array}{l}1=\text { Highly agree } \\
5=\text { Highly dis- } \\
\text { agree }\end{array}$ & 0.81 & 173 & 1.00 & 4.00 & 2.26 & 0.69 \\
\hline $\begin{array}{l}\text { Feeling of being lumbered-up } \\
\text { with the PSA job }\end{array}$ & 6 & $1-5$ & $\begin{array}{l}1=\text { Highly agree } \\
5=\text { Highly dis- } \\
\text { agree }\end{array}$ & 0.86 & 171 & 1.33 & 4.83 & 3.09 & 0.71 \\
\hline $\begin{array}{l}\text { Feeling of PSA as an extra- } \\
\text { burden next to other tasks }\end{array}$ & 6 & $1-5$ & $\begin{array}{l}\text { 1= Highly dis- } \\
\text { agree } \\
5=\text { Highly agree }\end{array}$ & 0.86 & 171 & 1.33 & 4.67 & 3.16 & 0.72 \\
\hline $\begin{array}{l}\text { Perception of lack of appro- } \\
\text { priate infrastructure and/or } \\
\text { material for PSA }\end{array}$ & 6 & $1-5$ & $\begin{array}{l}\text { 1= Highly dis- } \\
\text { agree } \\
5=\text { Highly agree }\end{array}$ & 0.79 & 173 & 1.00 & 4.50 & 2.97 & 0.72 \\
\hline $\begin{array}{l}\text { Worrying about the safety of } \\
\text { self-performed PSA }\end{array}$ & 5 & $1-5$ & $\begin{array}{l}\text { 1= Highly dis- } \\
\text { agree } \\
5=\text { Highly agree }\end{array}$ & 0.83 & 167 & 1.20 & 4.40 & 2.86 & 0.65 \\
\hline
\end{tabular}

PSA=Procedural Sedation/Analgesia. $\mathbf{N}=$ number of complete responses used for scale construction; $\mathbf{C} \boldsymbol{\alpha}=\mathbf{C r o n b a c h}$ alpha; Min=Lowest observed value; $\mathbf{M a x}=$ Highest observed value; $\mathbf{S D}=$ Standard Deviation

\section{Predictors of (Dis)Satisfaction with personal PSA practice $(N=155)$}

In the final regression model results only six of the nine newly-constructed scales of motivational beliefs turned out to have statistically significant effects on the level of (dis)satisfaction with PSA practice. Non-significant effects were found for the scales 'Self-evaluated lack of skills and knowledge for performing PSA', 'Evaluation of (supportive) attitude in diagnostic personnel' and 'Feelings of being lumbered-up with doing the job'. Univariate Pearson correlations of these three scales with the outcome variable were respectively: $r=0.546(p<0.001 ; n=148), r=0.326$ $(p<0.001 ; n=146)$ and $r=0.561(p<0.001 ; n=148)$. From the list of eight individual background variables and factors only 'personal use of a PSA protocol' finally turned out to have a statistically significant effect on the outcome variable in the final regression model. 
Table 2: Results of the final dummy regression analysis model using (dis)satisfaction of actually working with PSA as a 'dependent' variable. Listwise deletion of missing cases. Scores of the 'dependent' variable vary from 1 (highly satisfied) to 5 (highly dissatisfied). $N=144,11(7.1 \%)$ cases missing. Explained Variance $\left(r^{2}\right)=0.782$.

\begin{tabular}{|c|c|c|c|c|c|}
\hline \multirow[t]{2}{*}{ Predictors: } & \multicolumn{2}{|c|}{$\begin{array}{l}\text { Unstandardized } \\
\text { Coefficients }\end{array}$} & \multirow{2}{*}{$\begin{array}{c}\text { Standardized } \\
\text { Coefficients } \\
\text { Beta }\end{array}$} & \multirow[t]{2}{*}{$\mathrm{t}$} & \multirow[t]{2}{*}{$p$-value $e^{\S}$} \\
\hline & B & Std. Error & & & \\
\hline (Constant) & 2.449 & 0.515 & & 4.754 & $<0.001$ \\
\hline $\begin{array}{l}\text { Evaluation of (pos.)support by anaesthesiologists } \\
\text { (agree(1)-disagree(5)) }\end{array}$ & -0.216 & 0.112 & -0.334 & -1.931 & $(0.056)$ \\
\hline $\begin{array}{l}\text { Concern for PSA failure } \\
\text { (disagree(1)-agree(5)) }\end{array}$ & -0.060 & 0.139 & -0.066 & -0.432 & $(0.666)$ \\
\hline $\begin{array}{l}\text { Relevance of PSA-problem } \\
\text { (agree(1)-disagree(5)) }\end{array}$ & -0.198 & 0.045 & -0.217 & -4.370 & $<0.001$ \\
\hline $\begin{array}{l}\text { Feeling of PSA as extra-burden } \\
\text { (disagree(1)-agree(5)) }\end{array}$ & 0.171 & 0.044 & 0.188 & 3.911 & $<0.001$ \\
\hline $\begin{array}{l}\text { Lack of materials \& infrastructure } \\
\text { (disagree(1)-disagree(5)) }\end{array}$ & 0.008 & 0.082 & 0.009 & 0.095 & $(0.925)$ \\
\hline $\begin{array}{l}\text { Worrying about safety in PSA } \\
\text { (agree(1)-disagree(5)) }\end{array}$ & 0.168 & 0.050 & 0.169 & 3.357 & 0.001 \\
\hline $\begin{array}{l}\text { Use of protocol } \\
\text { (sometimes(1) versus never(0)) }\end{array}$ & -0.812 & 0.326 & -0.573 & -2.487 & $(0.014)$ \\
\hline $\begin{array}{l}\text { Use of protocol } \\
\text { (always(1) versus never(0)) }\end{array}$ & -0.979 & 0.308 & -0.750 & -3.176 & $(0.002)$ \\
\hline Evaluation support by anaesthetist * Concern for failure & 0.099 & 0.037 & 0.652 & 2.670 & 0.009 \\
\hline $\begin{array}{l}\text { Lack of materials * Use of protocol (sometimes(1) versus } \\
\text { never(0)) }\end{array}$ & 0.209 & 0.103 & 0.472 & 2.031 & 0.044 \\
\hline $\begin{array}{l}\text { Lack of materials * Use of protocol (always(1) versus } \\
\text { never(0)) }\end{array}$ & 0.271 & 0.099 & 0.629 & 2.730 & 0.007 \\
\hline
\end{tabular}

${ }_{\S}$ p-values between brackets should not be interpreted, due to higher order interactions of the predictor with others; * indicates a first-order interaction between two predictors

Using data on seven predictors the final regression model results comprise three main effects and two first-order interaction effects with statistical significance of $p<0.05$. (Table 2) Results can be described as follows:

1. If respondents believe that problems in PSA are a very relevant topic in Dutch paediatrics, they tend to feel significantly more dissatisfied about performing PSA than respondents who regard PSA problems as much less relevant. (beta=-0.217, $p<0.001$ )

2. If respondents regard PSA tasks as an extra burden next to their usual work, they will show significantly more dissatisfied feelings with PSA than respondents who do not regard PSA tasks as an extra burden. (beta $=0.188, \mathrm{p}<0.001)$

3. If respondents tend to worry about the safety of the PSA procedures, they will show significantly higher dissatisfaction with PSA than respondents who do not worry about safety. (beta $=0.169, p=0.001$ ).

4. If respondents express feelings of concern on risking failure in their PSA practice and -at the same time - negatively evaluate the collegial support they get (or do not get) from the anaesthesiologists at their hospital, they will show significantly higher scores of dissatisfaction than both effects separately added together (beta $=0.652, p=0.009$ ). When the total sample 
of respondents is artificially broken down into a group who shows no concern on risking failure in PSA practice ('Confident group'; score between 1.00 than $3.00, n=82,48.5 \%$ ) and a group expressing concern on risking failure ('Diffident group'; score from 3.00 to $5.00, n=87$, $51.5 \%$ ) the regression model can be repeated for both groups (6 or $3.4 \%$ has missing scores). In the 'confident' group the effect of the evaluation of the collegial support on (dis)satisfaction with PSA shows no statistical significance (beta $=0.030, p=0.730$ ), while in the 'diffident' group this effect turns out to be highly significant (beta=0.227, $p=0.005$ ).

5. If respondents express feelings of concern on the lack of materials and infrastructure they perceive in their PSA practice and -at the same time- profess to make personal use of protocols in their PSA practice, they will show significantly higher scores of dissatisfaction than both effects separately added together (respectively beta $=0.472, p=0.044$ for 'protocol sometimes used' versus 'protocol never used' and beta $=0.629, p=0.007$ for 'protocol always used' versus 'protocol never used'). So a specification effect is seen here: as a general result protocols users are satisfied with their PSA practice, unless they express feelings of shortcomings in materials and infrastructure. Then they are very dissatisfied with their PSA practice. When the total sample of respondents is broken down into a group who professes 'never' to use protocols in PSA ( $n=46,29.9 \%)$ and a group that says to use protocols 'occasionally, often or always' $(n=108,70.1 \%)$ the regression model can be repeated for both groups ( 21 or $12.0 \%$ has missing scores). In the no-protocol-user group the effect of feelings of concern on the lack of materials and infrastructure perceived in PSA practice shows no statistical significance (beta $=0.037, p=0.757$ ), while in the protocol-user group this effect turns out to be highly significant (beta $=0.278, p<0.001$ ).

Variance explained by the finally found regression model amounts to 0.782 .

\section{Discussion}

For many general paediatricians in The Netherlands, performing PSA in children turns out to be not a highly satisfying clinical activity. In this study we showed that the overall satisfaction on the state of affairs on self-performed PSA tends to vary considerably from specialist to specialist. (Table 1) This heterogeneity of opinions shows that performing PSA has become an "issue", a matter in which debate will rise and positions have been taken in. Some may wish to keep performing PSA in the familiar way, while others wish to change a lot. Studying the variations in (dis)satisfaction is quite interesting. Our final regression model, explaining $78.2 \%$ of satisfaction variance, found 5 significant effects ( 3 main effects and 2 interactive effects). (Table 2 ) Assuming a positive effect of feelings of satisfaction on professional performance, we believe that at least four of the 5 significant effects can be regarded as potential targets for a practice improving strategy in this population of PSA providers. We showed that motivational beliefs like 'the extra burden PSA-related tasks create next to the daily work' and 'worrying about the safety of the PSA procedures', are significantly related to the level of satisfaction on personal PSA performance. An implementation strategy that incorporates the sources of such positive and negative beliefs as strategic targets (i.e. diminishing the feeling or belief that PSA means an extra burden or might be unsafe) is likely to create a higher level of satisfaction. In addition, if respondents profess to make personal use of a protocol and - at the same time - express feelings of concern on the lack of materials and infrastructure they will show significantly higher scores of dissatisfaction. This means that an implementation strategy focusing only on the dissemination of PSA protocols will 
fail in the enhancement of satisfaction on PSA performance, if at the same time the belief of a lack of appropriate tools and infrastructure is neglected. This finding also suggests that offering protocols and theoretical frameworks to professionals may help discover additional shortcomings in the performance of PSA. Well-informed professionals are likely to become more faultfinding, critical and therefore less satisfied. Finally, and most interestingly, we found that in particular in less confident paediatricians the level of satisfaction is significantly related to the collegial support they get (or do not get) from anaesthesiologists. From a practical point of view this finding may help to direct specific interventions (e.g. enhancing anaesthetic support) to those professionals who have less confidence in their own competence.

In an earlier study in the same population we have recently shown that the adherence to safety guidelines on PSA is low. ${ }^{12}$ The lack of an appropriate, well-tailored program for guideline implementation is likely to be an important explanation. In modern medicine, guidelines are increasingly being designed in an attempt to improve the professional performance, health care process, outcomes and costs. However, it has also been shown that the mere designing, publishing and disseminating of guidelines do not necessarily imply the intended positive change in daily practice unless they are clear and reflect the clinical context. ${ }^{14}$ Guideline recommendations land into the quagmire of a heterogeneous population of professionals having different background characteristics, experience, knowledge and skills. In addition they certainly have differently developed opinions and motivational beliefs (i.e. positive and negative perceptions, evaluations and expectations). This bewildering heterogeneity of factors to be reckoned with in upgrading the present situation is nicely demonstrated in this study. Since all these factors separately may act as important facilitators as well as barriers for guideline implementation, a thorough assessment of them and their interactive effects will be highly informative for the design of a successful implementation strategy.

This study has several limitations. As in any self-report survey, data may be subject to social desirability bias. The 'one-shot' approach of the sample survey technique is another limitation. Forming of opinions is a dynamic process, and even then opinions may change over time. A more longitudinal approach of registering (dis)satisfaction, underlying beliefs and even background characteristics can be done when fixed panel-studies are used. To register progress in implementation studies such an approach is a methodological prerequisite. Next, beliefs cannot be operationalized and quantified in an interval or ratio scale, so results are basically of an ordinal nature. Practical application of parametric statistics must therefore always be regarded with caution. Finally, the basic assumption that actual adherence behaviour is determined by feelings of (dis)satisfaction, motivational beliefs and background characteristics needs to be further examined in future research.

In conclusion we showed that in Dutch general paediatricians the level of satisfaction varies largely from specialist to specialist. We showed that the sources of these differences of opinion are lying in the individual background characteristics and underlying motivational beliefs or concerns. These insights can be used as targets for the enhancement of guideline adherence. Guideline implementation is a complex process that should at least make use of an individually focussed, non-uniform approach of a - by definition - heterogeneous target population. An implementation strategy that incorporates sources for dissatisfaction as strategic targets is very likely to be more successful. 


\section{References}

1. Krauss B, Green SM. Procedural sedation and analgesia in children. Lancet. 2006;367(9512):766-780.

2. Cote CJ. Discharge criteria for children sedated by nonanesthesiologists: is "safe" really safe enough? Anesthesiology. 2004;100(2):207-209.

3. Guidelines for monitoring and management of pediatric patients during and after sedation for diagnostic and therapeutic procedures: addendum. Pediatrics. 2002;110(4):836-838.

4. Practice guidelines for sedation and analgesia by non-anesthesiologists. Anesthesiology. 2002;96(4): 1004-1017.

5. SIGN Guideline 58: Safe sedation of children undergoing diagnostic and therapeutic procedures http://www.sign.ac.uk/pdf/sign58.pdf. Published 2004.

6. Guideline statement: management of procedure-related pain in children and adolescents. $J$ Paediatr Child Health. 2006;42 Suppl 1:S1-29.

7. Cote $\mathrm{CJ}$, Wilson S. Guidelines for monitoring and management of pediatric patients during and after sedation for diagnostic and therapeutic procedures: an update. Pediatrics. 2006;118(6):2587-2602.

8. Leroy $\mathrm{PL}$, Gorzeman MP, Sury MR. Procedural sedation and analgesia in children by nonanesthesiologists in an emergency department. Minerva pediatrica. 2009;61(2):193-215.

9. Barbi E, Gerarduzzi T, Marchetti F, Neri E, Verucci E, Bruno I, et al. Deep sedation with propofol by nonanesthesiologists: a prospective pediatric experience. Archives of pediatrics \& adolescent medicine. 2003;157(11):1097-1103.

10. Hoffman GM, Nowakowski R, Troshynski TJ, Berens RJ, Weisman SJ. Risk reduction in pediatric procedural sedation by application of an American Academy of Pediatrics/American Society of Anesthesiologists process model. Pediatrics. 2002;109(2):236-243.

11. Pitetti R, Davis PJ, Redlinger R, White J, Wiener E, Calhoun KH. Effect on hospital-wide sedation practices after implementation of the 2001 JCAHO procedural sedation and analgesia guidelines. Archives of pediatrics \& adolescent medicine. 2006;160(2):211-216.

12. Leroy PL, Nieman FH, Blokland-Loggers HE, Schipper DM, Zimmermann LJ, Knape JT. Adherence to safety guidelines on paediatric procedural sedation: the results of a nationwide survey under general paediatricians in The Netherlands. Archives of disease in childhood. 2010.

13. Wallace JE, Lemaire JB, Ghali WA. Physician wellness: a missing quality indicator. Lancet. 2009;374 (9702):1714-1721.

14. Sheldon TA, Cullum N, Dawson D, Lankshear A, Lowson K, Watt I, et al. What's the evidence that NICE guidance has been implemented? Results from a national evaluation using time series analysis, audit of patients' notes, and interviews. BMJ (Clinical research ed. 2004;329(7473):999. 


\section{Appendix A: Schematic overview of study domains: general and specific beliefs in experiencing and evaluating one's own performance in PSA.}

\begin{tabular}{ll}
\hline Concept & Content \\
\hline General belief & \\
$\begin{array}{ll}\text { A. Overall satisfaction or dissat- } \\
\text { isfaction with own perform- } \\
\text { ance }\end{array}$ & $\begin{array}{l}\text { The respondent's positive or negative attitude towards the } \\
\text { state of affairs in an average self-performed PSA procedure } \\
\text { during the } 12 \text { months preceding the answering of the } \\
\text { questionnaire. }\end{array}$
\end{tabular}

Specific motivational beliefs ( $N=11$ )

B. Level of Personal Knowledge

The respondent's perception that he/she is or is not lacking in practical knowledge in performing a PSA

C. Importance \& Relevance of the problem

The respondent's evaluation of the rele-

vance/importance/urgency of the problem of PSA in Dutch paediatrics

D. Worrying about safety and risks

The extent in which the respondent is worrying about the safety of the PSA procedure and the risks involved in performing

Type

Overall evaluation

E. Confidence in own skills Evaluation of one's competence /the extent of confidence one has in own skills regarding PSA

F. Confidence in drug effectiveness

The respondent's perception of the effectiveness of drugs used in PSA.

G. Feeling of Extra burden

The expectations the respondent has on the degree of workload PSA represents amongst the other tasks one has to perform as a general paediatrician

H. Lack of infrastructure and materials

The respondent's perception on the availability of infrastructure and materials needed for performing PSA properly

I. Feelings of concerns, anxiety and stress

Feelings of anxiety and stress the respondent may have when considering failure in performing PSA

Perception

Overall evaluation

Perception /

Expectation

Expectation

Perception

Expectation

Perception

Expectation /

Anxiety

Expectation of success or failure the respondent has in performing PSA

Expectation

Expectation

K. Feeling of being lumbered up Feelings the respondent may have of being lumbered up with PSA task with the responsibility for the task of PSA at his/her department

L. Level of support by other professionals

Evaluation of support given by professional co-workers in performing PSA (i.e.: anaesthesiologists, diagnostic personnel). 



\section{CHAPTER 3}

\section{Professional skills and competence for safe and effective Procedural Sedation/Analgesia (PSA) in children}

Recommendations based on a systematic review of the literature

This chapter has been published in:

Piet LM Leroy, Daphne M Schipper, Hans(J) TA Knape. Professional skills and competences for safe and effective procedural sedation in children: recommendations based on a systematic review of the literature. Int J Paediatr 2010; 3: 188-191 


\section{Abstract}

Objectives: To investigate which skills and competence are imperative to assure optimal effectiveness and safety of procedural sedation/analgesia (PSA) in children and to analyze the underlying levels of evidence.

Study Design and methods: Systematic review of relevant literature published between 1993 and March 2009. Selected papers were classified according to their methodological quality and summarized in evidence-based conclusions. Since no randomized prospective studies comparing different levels of skills and competence were found, indirect techniques were used to deduce from literature which competences and skills are essential to guarantee optimal safety and effectiveness of PSA in children.

Results: Although the safety profiles vary among PSA drugs, the possibility of potentially serious adverse events and the predictability of depth and duration of sedation define the imperative skills and competence necessary for a timely recognition and appropriate management. The level of effectiveness is mainly determined by the ability to apply titratable PSA, including deep sedation using short-acting anaesthetics for invasive procedures and nitrous oxide for minor painful procedures, and the implementation of non-pharmacological techniques.

Conclusions: PSA related safety and effectiveness are determined by the circumstances and professional skills rather than by specific pharmacologic characteristics. Evidence based recommendations regarding necessary skills and competence should be used to set up training programs and to define which professionals can and cannot be 'credentialed' for PSA in children. 


\section{Introduction}

Invasive diagnostic procedures are a part of daily paediatric practice. Many of these procedures are painful, stressful and impossible to perform without immobilizing the patient. Therefore, procedural sedation/Analgesia (PSA) is required to enable these procedures to be performed. PSA can be defined as the use of sedative, analgesic, or dissociative drugs in order to provide anxiolysis, analgesia, sedation, and motor control during painful or unpleasant procedures. ${ }^{1}$

Since anaesthesiologists cannot cover the growing demand for PSA, non-anaesthesiologists have organized their own PSA strategies. ${ }^{2,3}$ Historically, this resulted in a wide range of drugs and techniques for use in paediatric PSA, involving a large variance of sedation levels, sedation level predictability, effectiveness and associated risks. However, by the end of last century PSA by non-anaesthesiologists was increasingly criticized by anaesthesiologists for neglecting transparency and standard safety precautions. There are strong indications that within this criticism, a source could be found for PSA related accidents. ${ }^{4,5}$ About a decade ago, dedicated nonanaesthesiology specialists who recognized the urgent need to improve the safety and quality of PSA in children joined the initial criticism by anaesthesiologists, pointing at the safety problems of PSA by the untrained. In order to prevent PSA-related tragedies guidelines on PSA were published. ${ }^{1,6-10}$ In summary, these guidelines specify safety precautions that include the assessment of the risk of sedation prior to PSA, informed consent, guidelines on proper fasting status, appropriate monitoring, recovery standards, appropriate rescue facilities and specific professional skills and competence. Generally recommended skills and competence are: the ability to perform a pre-procedural risk analysis, practical knowledge and experience of applied sedatives, the ability to implement the necessary monitoring and surveillance, the ability to recognize and interpret sedation levels and the ability to immediately recognize and adequately treat any unwanted side effects or complications, particularly hypoventilation and airway obstruction. These recommendations are mainly based on indirect evidence, expert opinion, 'common sense' and widely accepted safety rules for general anaesthesia. The adoption of a uniform and systematic practice is associated with a significant reduction in adverse events during anaesthesia. ${ }^{11}$ Similarly, there is strong evidence that implementation of published guidelines leads to safer and more effective PSA. ${ }^{12-15}$

However, despite the availability of guidelines, PSA practice is still unsafe in many settings and adherence to guidelines among non-anaesthesiologists has been reported to be low. ${ }^{16,17}$ It has been argued that guidelines on PSA produced by consensus between anaesthesiologists (rather than on evidence-based guidelines by the clinicians themselves) have caused confusion and variation in practices. ${ }^{3}$

Recent papers focus increasingly on the duty to deliver effective PSA, not only from a procedural point of view (i.e. guaranteeing predictable procedural success and timing) but also from a patient's perspective (i.e. achieving optimal procedural comfort and minimizing procedural stress and failure). ${ }^{18,19}$ Drugs traditionally used for PSA (e.g. chloral hydrate, midazolam, barbiturates and lytic cocktails) are associated with a substantial risk of procedural failure, discomfort, extended sedation times and deeper sedation levels than intended with associated safety risks. ${ }^{20,21}$ Patient comfort is currently considered a primary goal of procedural sedation. ${ }^{22}$ It has been argued that young children who are anticipated to suffer from substantial emotional distress need a titrated form of PSA, including deep sedation, in order to have a successfully completed procedure, and to avoid major psychological trauma to the child, the family and health- 
care staff. ${ }^{23,}{ }^{24}$ The application of forced immobilization by physical restraint is increasingly considered as inhumane and unacceptable in non-lifesaving procedures ${ }^{22,25}$.

We searched the literature for available evidence on essential professional skills and competence required for effective and safe PSA in children. Results were used to define evidencebased recommendations on the skills and competence a professional entrusted with PSA should minimally possess, in order to be able to perform PSA in children safely and effectively.

\section{Methods}

Literature was searched and selected by a multidisciplinary panel of the Dutch Institute for Health Care Improvement $\mathrm{CBO}$, involved in the development of an evidence-based guideline on PSA using the Evidence Based Guideline Development (EBRO) methodology. Systematic searches were done in Medline, Cochrane Library and Embase, using the Medical Subject Heading (MESH) search-terms "Conscious sedation/all", "Moderate sedation/all" "Deep sedation/all" and the free search-terms "sedation", "paediatric sedation" and "procedural sedation" in title or abstract. The search was limited to papers published between 1993 and March 2009, in 4 languages (Dutch, English, French and German) and to human subjects aged 0-18 years. Results were systematically and repeatedly combined with the MESH-term 'Drug Toxicity' and the MESH-terms of drugs, drug combinations and drug groups available for PSA (chloral hydrate, (lytic) cocktails, promethazine, chlorpromazine, pentobarbital, thiopental, midazolam, fentanyl, meperidine, ketamine, propofol, dexmedetomidine, remifentanil, nitrous oxide, opioids, benzodiazepines, antihistaminic, antipsychotics, barbiturates, nitrous oxide and anaesthetics). For all drugs specific searches were done using the MESH subheading 'adverse effects'. Additional combined searches were done using search terms for safety, effectiveness and non-pharmacologic methods (hypnosis, distraction techniques, play therapy). Textbooks and reference tables were systematically searched for additional papers.

Before inclusion in the pool of studies to be reviewed, all papers obtained were analyzed by the multidisciplinary panel for relevance and accuracy of definitions of safety and effectiveness.

In accordance with the EBRO methodology selected papers were classified according to their methodological quality and strength of evidence: A1: systematic review including at least two independent A2-level studies, A2: Randomized, double blinded comparative clinical trial of good quality and substantial size, B: comparative study, including retrospective cohort study and case-controlled trial, but not having all characteristics of an A2 study, C: non-comparative studies and D: expert opinion. Findings from literature were summarized in conclusions. These conclusions were classified in 4 levels. Level 1: conclusion based on one A1 study or on at least two independent $A 2$ studies, Level 2: conclusion based on one A2 study or on at least two independent B studies, Level 3: conclusion based on one B or C study, Level 4: conclusion based on expert opinion only. Finally, on the basis of the conclusions and remaining considerations (nonclassified) recommendations were formulated. 


\section{Results}

\section{Requisite skills and competence to guarantee optimal safety}

Many studies were found claiming the safety of all kinds of PSA drugs in a variety of settings but usually in a limited series of patients. However, given an estimated incidence of severe adverse events of about $1 / 10000$, the majority of these studies are insufficiently powered to prove such conclusion. ${ }^{18}$ Most studies use vague definitions for the adverse reactions they report and consider the absence of directly life-threatening events as synonym for 'safe'. In more recent observational studies on PSA, the study setting is usually a strictly controlled, well-equipped, welltrained and dedicated sedation team, which may differ appreciably from common settings in many practices around the world. Finally, well-designed controlled prospective studies in nonanaesthesiologists analyzing the relationship between the level of professional skills/competence and the safety of PSA are non-existent. Therefore evidence on this subject must be gathered in an indirect way. To do so the following rationale was followed. At first, published critical analysis of PSA related incidents might elucidate the requisite competence and skills for PSA. Next, the level of skills and competence professionals must achieve with regard to safety are likely to be determined by (1) the probability that a medicine may have undesirable adverse effects which require specific recognition and treatment, and (2) the predictability of the depth and duration of sedation of a medicine. The latter is important since unexpected deep sedation is associated with a higher rate of adverse events. ${ }^{21}$ Out of all retrieved studies reporting PSA related adverse events, only those were selected for this systematic review that reported the incidence of adverse events in large numbers of patients ( $> \pm 1000)$, or that had studied adverse events following the use by non-anaesthesiologists of the anaesthetics propofol, ketamine, dexmedetomidine, remifentanil and nitrous oxide.

\subsection{Retrospective Critical Incident analysis of PSA related adverse events and outcomes}

In 2000, Coté published, in two separate papers, a retrospective critical incident analysis of adverse sedation events in paediatrics, as reported to the American Food and Drug Administration between 1969 and 1996. 95 incidents were reported, 51 resulting in death, 9 in permanent neurological injury and 21 in prolonged hospitalization. Significant contributing factors were: 'out of hospital' locations, inappropriate monitoring of physiological parameters, inadequate resuscitation skills, inadequate pre-sedation medical evaluation and inadequate recovery procedures. No particular medication was associated with a higher risk, except that overdosing and drug interactions (particularly when 3 or more drugs were used) were associated with mortality. ${ }^{4,5}$ Although the safety profile and the margins of safety vary among drugs, Coté showed that PSA related safety is determined by circumstances and professional skills, rather than by specific pharmacological characteristics. Professionals who do not have the requisite competence to recognize and treat the potential PSA-related complications constitute a significant risk factor for the occurrence of fatal complications or complications causing permanent harm to the patient. (Level 3 conclusion; ${ }^{4,5}$ )

\subsection{Reported data on PSA related adverse events}

The studies stated below are summarized in table 1 'Overall conclusions regarding the relation between professional competence/skills and PSA related safety' and table 2 'Drug-specific conclusions regarding the relation between professional competence/skills and PSA related safety'. 
Table 1: Overall conclusions regarding the relation between professional competence/skills and PSA related safety.

\begin{tabular}{lll}
\hline $\mathrm{Nr}$ Conclusion & Quality \\
Level
\end{tabular}

1 Serious PSA related adverse events occur more frequently

I. In children with an underlying disease.

Level 1
A1 Green $2009^{42}$
B Sanborn $2005^{26}$, Cravero $2009^{42}$
C Malviya $1997^{27}$, Vespasiano $2007^{41}$

II. If multiple sedatives are used

Level 1
A1 Green $2009^{46}$
B Hoffman 2002, Pitetti 2003, Sanborn $2005^{26}$, Cravero $2009^{42}$
C Gall $2001^{58}$

\section{In young children}

Level 1
A1 Green $2009(<2 \text { years })^{46}$
B Cravero 2009 (<6 months) ${ }^{42}$
C Malviya $1997(<1 \text { year })^{27}$, Gall $2001(<1 \text { year })^{58}$

\section{In certain drugs compared to others:}

IV.1. The combination of a benzodiazepine with an opiate (for example midazolam + fentanyl) is associated with a higher risk of respiratory complications (21-23\%) compared to the use of midazolam alone or ketamine with midazolam.

A2 Yldzdaz $2004^{8}$

B Pitetti $2003^{35}$, Roback $2005^{32}$, Newman $2003^{33}$

IV.2. Oral pentobarbital is associated with less adverse events compared to oral chloral hydrate B Mason $2004^{29}$

IV.3. In comparison with ketamine, midazolam and ketamine + midazolam, midazolam + fentanyl and propofol generate a higher risk of hypoventilation and desaturation.

A2 Yldzdaz $2004^{8}$

2 Serious PSA related adverse events occur less frequently if specifically trained professionals working in dedicated teams perform sedation according to international guidelines.
B Barbi $2003^{12}$, Hoffman $2002^{13}$, Cravero $2009^{42}$
C Vespasiano $2007^{41}$ 
Table 2: Drug-specific conclusions regarding the relation between professional competence/skills and PSA related safety.

\begin{tabular}{ll}
\hline $\mathrm{Nr}$ Conclusion & $\begin{array}{l}\text { Quality } \\
\text { Level }\end{array}$ \\
\hline
\end{tabular}

\section{Non-titratable drugs intended for moderate to deep sedation}

1 During PSA, intended to moderate or deep sedation, with the use of benzodiazepines, chloral Level 2 hydrate, barbiturates, opiates or combinations of these medicines, and during the subsequent recovery phase, there exists a variable but real risk of potentially serious drug-induced adverse events. Especially the risk for respiratory depression and/or airway obstruction necessitates specific skills and competence from the professionals in charge in terms of recognition and treatment.
B Hoffman $2002^{13}$, Pitetti $2003^{35}$, Sanborn $2005^{26}$, Cravero $2006^{28}$, Roback $2005^{32}$, Newman $2003^{33}$, Pena $1999^{34}$, Mason $2001^{31}$, Mason $2004^{29}$, Mason $2004^{30}$
C Malviya $1997^{27}$

\section{Propofol}

1 During PSA using propofol, there is a real risk of potentially serious drug-induced adverse events. Especially the risk for respiratory depression and/or airway obstruction necessitates specific skills and competence from the professionals in charge in terms of recognition and treatment.

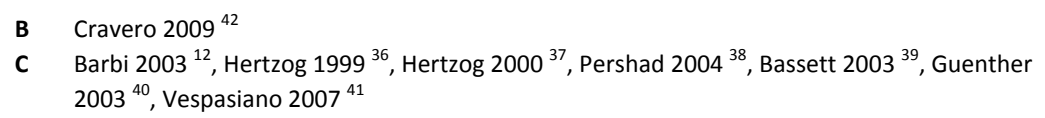

2 PSA with propofol, including deep sedation, is equally safe in the hands of anaesthesiologists and non-anaesthesiologists if the latter are well trained and part of dedicated sedation team.
B Cravero $2009^{42}$
C Barbi $2003^{12}$, Vespasiano $2007^{41}$

3 A deep PSA using ketamine or propofol for examination of the upper airways, or for endoscopies of the upper gastro-intestinal system, carries a real risk of potentially serious complications (i.e. laryngospasm and deep desaturation), which require specific skills and competence from the professionals in charge in terms of recognition and treatment.

C Barbi $2003^{12}$, Green $2001^{47}$

\section{Ketamine}

1 During PSA using ketamine, there is a small but real risk of potentially serious drug-induced adverse events. Especially the risk for respiratory depression, airway obstruction and - infrequently - laryngeal spasm necessitates specific skills and competence from the professionals in charge in terms of recognition and treatment.
A1 Green $2009^{46}$
C Green $2001^{47}$, Evans $2005^{43}$, Meyer $2003^{45}$, Cheuk $2005^{44}$,

2 Independent risk factors for respiratory adverse events during a PSA with the use of ketamine are Level 1 high intravenous doses, administration to children younger than 2 years or aged 13 years or older, and the co-administration of anticholinergics or benzodiazepines. 
Table 2: Drug-specific conclusions regarding the relation between professional competence/skills and PSA related safety (Continued)

\begin{tabular}{ll}
\hline $\mathrm{Nr}$ Conclusion & $\begin{array}{l}\text { Quality } \\
\text { Level }\end{array}$ \\
\hline
\end{tabular}

\section{Dexmedetomidine}

1 Based on a limited published experience on the use of dexmedetomidine for PSA by experienced Level 1 professionals, there seems to be a very small risk of potentially serious drug-induced adverse events. Respiratory events are extremely rare and hemodynamic adverse events (i.e. bradycardia and hypotension) are mostly clinically insignificant. Specific experience in dosing techniques, individual titration and avoiding dexmedetomidine in those patients who may not tolerate hemodynamic fluctuations seems to be associated with low risks.

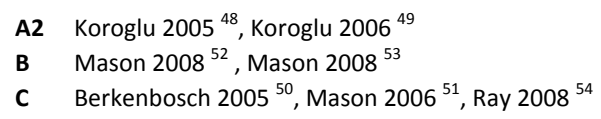

\section{Remifentanil}

2 During PSA using remifentanil, there is a real risk of potentially serious drug-induced adverse events. Especially the risk for respiratory depression and/or airway obstruction necessitates specific skills and competence from the professionals in charge in terms of recognition and treatment.
A2 Keidan $2001^{56}$
C Litman $1999^{104}$, Litman $2000^{55}$

\section{Nitrous Oxide}

1 PSA with nitrous oxide is associated with an extremely low chance of serious adverse events. Instant discontinuation of gas flow in case of respiratory depression is the most important rescue intervention.
B $\quad$ Babl $2005^{60}$, Babl $2008^{61}$
C Gall $2001^{58}$

2 Specific risks for adverse events during nitrous oxide administration are: I. A young age $(<1$ year old)

\section{Gall $2001^{58}$}

II. Simultaneous use of other sedatives

\section{Gall $2001^{58}$}

3 In patients sedated with nitrous oxide, there exists no significant difference in median fasting time between patients with and without emesis

\section{B Babl $2005^{60}$}

4 Nitrous oxide $70 \%$ causes significantly deeper sedation compared to nitrous oxide $50 \%$. However, Level 3 if embedded in a comprehensive sedation program there exists no significant difference in adverse events rates between both regimens.
B Babl $2008^{61}$
C $\quad$ Zier $2007^{59}$ 
Adverse effects of commonly used non-titratable sedatives

A retrospective study by Sanborn et al. of 16467 sedations during imaging procedures in children using chloral hydrate, midazolam, fentanyl or pentobarbital found $70(0.4 \%)$ respiratory incidents: desaturation only $(\mathrm{N}=58)$, aspiration $(\mathrm{N}=2)$ and airway obstruction requiring airway intervention $(\mathrm{N}=10)$. The main risk factors were an underlying respiratory problem and the use of more than one sedative. ${ }^{26}$

A prospective study by Malviya et al. in 1140 children, of which the majority were sedated with chloral hydrate for diagnostic imaging, showed a $5.5 \%$ incidence of respiratory complications leading to oxygen saturation of $<90 \%$ : respiratory depression $(4.7 \%)$, airway obstruction $(0.6 \%)$ and apnea $(0.17 \%)$. The risk of complications was significantly greater for more seriously ill children and for children less than one year old. ${ }^{27}$.

A risk analysis by Hofmann et al. based on prospectively collected data of 950 sedations using chloral hydrate, midazolam, fentanyl, pentobarbital, ketamine or cocktails of 3 or more agents, identified 27 sessions (2.8\%) in which a serious adverse event occurred: deep desaturation $(\mathrm{N}=9)$, airway obstructions $(\mathrm{N}=5)$, apneas $(\mathrm{N}=3)$, aspirations $(\mathrm{N}=2)$, hypotension or bradycardia $(N=2)$, excessively deep or prolonged sedations $(N=6)$. Significant risk factors were the absence of a systematic risk assessment, a failure to follow safety guidelines, deep sedation, the simultaneous use of multiple agents and the use of chloral hydrate. ${ }^{13}$.

A prospective international multi-centre study of 30037 sedations by specifically trained professionals working in dedicated PSA teams reported low incidences of major adverse events: desaturation (SatO2 < 90\%) 1.57\%, stridor $0.04 \%$, laryngospasm $0.04 \%$, apnea $0.24 \%$, excessive airway secretions $0.41 \%$ and vomiting $0.47 \%$. The attending professional could adequately treat all complications. Cardio-pulmonary resuscitation was necessary in one case. Anaesthesiologists (19\%), emergency physicians (28\%) and intensivists (28\%) administered the sedations. The most frequently used sedatives were propofol $(50 \%)$, midazolam $(27 \%)$, ketamine (14\%), chloral hydrate (12\%), pentobarbital (13\%) and opiates $(10 \%){ }^{28}$

Mason et al reported in three separate comparative studies the adverse events of oral and intravenous (IV) pentobarbital used for PSA in diagnostic imaging or nuclear medicine. Potentially severe adverse events like oxygen desaturation occurred extremely rare $(<1 \%)$. Compared to oral chloral hydrate and intravenous pentobarbital, oral pentobarbital is associated with significantly less desaturations (respectively $1.6 \%$ versus $0.2 \%$ and $0.9 \%$ versus $0.2 \%$ ) ${ }^{29-31}$

A retrospective study by Roback et al. in 2500 successive children undergoing PSA in an emergency department (ED) showed that the incidence of respiratory complications depended on the medication used: $5.8 \%$ for midazolam, $6.1 \%$ for ketamine, $10 \%$ for ketamine + midazolam, and $19.3 \%$ for midazolam + fentanyl. ${ }^{32}$.

A prospective study by Newman et al. of 1341 PSA sessions in children in an ED showed an incidence of serious complications of $11.9 \%$ ( $96,2 \%$ hypoxia, $1,3 \%$ hypotension and $2,5 \%$ stridor). $92 \%$ of the complications occurred during the actual procedure, whereas the rest occurred after the procedure up to 40 minutes after the last dose of sedative. The risk of complications depended strongly on the medication used: midazolam $1.4 \%$, ketamine + midazolam + atropine 9.8\% and midazolam + fentanyl $21.5 \% .^{33}$

Another ED study by Pena et al. of 1180 successive children, using intravenous medicines (midazolam+fentanyl $\mathrm{N}=391$, midazolam $\mathrm{N}=67$, fentanyl $\mathrm{N}=21$, ketamine $\mathrm{N}=40$, pentobarbital $\mathrm{N}=93$, lorazepam $\mathrm{N}=9$ or midazolam+morphine $\mathrm{N}=1)$, intramuscular ketamine $(\mathrm{N}=180)$, oral medicines (midazolam $\mathrm{N}=62$, ketamine $\mathrm{N}=2$, chloral hydrate $\mathrm{N}=122$, diazepam $\mathrm{N}=1$, lorazepam $\mathrm{N}=1$ ), rectal chloral hydrate $\mathrm{N}=4$ ), intranasal medicines (midazolam $\mathrm{N}=3$, midazolam+sufentanil $\mathrm{N}=25)$, and nitrous oxide $(\mathrm{N}=168)$, showed an overall complication incidence of $2.3 \%(\mathrm{~N}=27)$. The 
following complications occurred: desaturation $<90 \%$ requiring intervention $(N=10)$, apnea $(\mathrm{N}=1)$, larynx spasm $(\mathrm{N}=1)$, bradycardia $(\mathrm{N}=1)$, stridor with vomiting $(\mathrm{N}=1)$ and 1 child started to vomit while being ventilated with a mask/bag applied to treat desaturation. There was no significant difference in the incidence of adverse events between the different sedation medicines. $^{34}$

A prospective study by Pitetti et al. of 1244 sedations in 1215 children in an ED showed an incidence of adverse events of $17.8 \%$ including desaturation ( $N=178)$, stridor $(\mathrm{N}=6)$, hypotension $(\mathrm{N}=2)$, vomiting $(\mathrm{N}=4)$, a rash $(\mathrm{N}=7)$, agitation $(\mathrm{N}=9)$, hiccups $(\mathrm{N}=3)$ and dizziness $(\mathrm{N}=3)$. An antidote had to be administered 6 times ( 3 flumazenil, 3 naloxone) and 2 patients sedated with fentanyl + midazolam required respiratory interventions (one with a Mayo cannula and one with mask/bag ventilation). The risk of complications depended heavily on the medication used. Patients sedated with midazolam + fentanyl had a significantly higher risk of adverse events $(161 / 686=23.4 \%)$, compared to patients who had been treated with midazolam + ketamine + atropine $(24 / 277=8.6 \%)$ or IV midazolam $(1 / 65=1.5 \%) .{ }^{35}$

In a randomized controlled trial by Yldzdaz et al. of 126 children undergoing a PSA for painful oncology procedures patients were randomly assigned for one of five forms of intravenous PSA: ketamine $(1 \mathrm{mg} / \mathrm{kg})$, midazolam $(0.15 \mathrm{mg} / \mathrm{kg})$, ketamine + midazolam $(1 \mathrm{mg} / \mathrm{kg}+0.1$ $\mathrm{mg} / \mathrm{kg})$, midazolam + fentanyl $(0.1 \mathrm{mg} / \mathrm{kg}+2$ micrograms $/ \mathrm{kg})$ and propofol $(2 \mathrm{mg} / \mathrm{kg})$. Patients were monitored through saturation measurement and capnography. Patients sedated with midazolam + fentanyl and with propofol had a significantly more desaturations and hypercapnia compared to the three other groups. Desaturations were observed in $0 \%, 0 \%, 8 \%, 28 \%$ and $52 \%$ respectively, whereas hypercapnia was found in $0 \%, 0 \%, 0 \%, 4 \%$ and $12 \%$ respectively. $^{8}$

\section{Adverse effects of Propofol}

Barbi's prospective study concerned deep PSA with propofol administered by nonanaesthesiologists (1059 procedures in 827 children aged $0-21$ years old: gastroscopies $(\mathrm{N}=483)$, colonoscopies $(\mathrm{N}=289)$ and painful procedures $(\mathrm{N}=173)$. All sedating professionals had followed a specific training, including theoretical and practical training on propofol, airway management, mask/bag ventilation and resuscitation. Of the 1059 patients, 34 (12.6\%) had a transient desaturation that resolved spontaneously. Deep desaturation with the need for mask/bag ventilation was required in $4 / 483$ patients $(0.8 \%)$ undergoing a gastroscopy, in $1 / 287$ patients $(0.3 \%)$ undergoing a painful intervention and in $0 / 289$ patients $(0.0 \%)$ undergoing a colonoscopy. Laryngospasm occurred in 10/483 patients (2.1\%) who underwent a gastroscopy. In 24 of the 483 gastroscopies (4.9\%) an anaesthesiologist was urgently required. In $13 / 24$ cases $(54.2 \%)$ this concerned assistance with the laryngoscopic insertion of an endoscope, in 10/24 cases (41.7\%) the treatment of a laryngospasm and in $1 / 24$ cases (4.2\%), assistance to deal with a serious esophageal bleed. The trained professionals were able to manage adequately all adverse events that occurred during colonoscopies and painful interventions. ${ }^{12}$ Propofol for PSA in children administered by specifically trained non-anaesthesiologists has also been studied in paediatric oncology, radiology and emergency medicine. A retrospective study by Hertzog et al. found that in 251 propofol sedations by paediatric intensivists hypotension (50\%) and respiratory depression requiring transient bag-valve-mask ventilation (6\%) were the most important adverse events. ${ }^{36}$ A prospective study by the same authors in 28 oncology patients, undergoing 50 sedations, showed similar results: transient hypotension (64\%) and partial airway obstruction (12\%) were the most important adverse events. Apnea requiring bag-valve-mask ventilation occurred in $2 \%$ of procedures. ${ }^{37}$ In a retrospective case series by Pershad et al. $(\mathrm{N}=52)$ of propofol PSA in the ED, no patient required assisted ventilation or developed clinically significant hypotension. 
The incidence of respiratory depression requiring airway repositioning or supplemental oxygen was $5.8 \%{ }^{38}$

Bassett et al. analyzed prospectively 293 propofol sedations in children on an ED. Transient decrease in systolic blood pressure without clinical signs of poor perfusion was found in $92 \%$ of the patients. Nineteen patients (5\%) had hypoxia, 11 patients $(3 \%)$ required airway repositioning or jaw-thrust maneuvers, and 3 patients $(0.8 \%)$ required bag-valve-mask ventilation. No patient required endotracheal intubation. ${ }^{39}$ In a similar study by the same authors in 87 patients (291 sedation sessions) partial airway obstruction requiring brief jaw-thrust maneuver was noted for $4 \%$ of patient sedations. Transient apnea requiring bag-valve-mask ventilation occurred in $1 \%$ of patient sedations. ${ }^{40}$

Vespasiano et al. reported a prospective study on 7304 propofol sedations outside the operation room in 4464 children, undergoing MRI (42.8\%), non-MRI diagnostic imaging (22.5\%), hematology/oncology procedures $(26.2 \%)$ or other procedures $(10.5 \%)$. All sedations were performed by paediatric intensivists according a sedation program that was in adherence to the American Academy of Paediatrics guidelines. The program was locally governed by a multidisciplinary committee with representation from anaesthesiology, critical care, nursing, oncology, cardiology and emergency medicine. To assess the overall safety profile of propofol a specific quality audit tool was designed. Hypotension ( $>25 \mathrm{mmHg}$ drop from baseline) occurred in 31.4 $\%$ of the patients but was mostly without circulatory compromise. High volume fluid therapy was necessary in only $0.11 \%$ of cases. Infrequent respiratory adverse events were laryngospasm (0.27\%), regurgitation without aspiration $(0.05 \%)$, regurgitation with aspiration $(0.01 \%)$ and bronchospasm $(0.15 \%)$. Almost $5 \%$ of patients had an oxygen desaturation ( $1.73 \%$ between $85-$ $90 \% ; 2.9 \%<85 \%$ ) while airway obstruction requiring an oral or nasal airway occurred in $2 \%$ of cases. Unfortunately $\mathrm{ETCO}_{2}$ was not evaluated systematically in this study. Only $0.37 \%$ of the patients needed bag-valve-mask ventilation because of hypopnea and/or apnea. All side effects could be managed successfully by the sedation team. There were no cardiac arrests. Patients with an abnormal airway (as defined by an airway score) were significantly more likely to develop oxygen desaturation or airway obstruction. None of the intended procedures or sedations had to be aborted. ${ }^{41}$

The multicentric Paediatric Sedation Research Consortium (PSRC) collected prospectively data on 49836 propofol sedations in children. The PSRC consists of anaesthesiologists, paediatric medical subspecialists, emergency physicians, paediatric intensivists, nurses, physician assistants and health care research personnel who seek to continuously improve the quality, safety, effectiveness and cost of paediatric sedation/anaesthesia practice. Participants work in 37 different locations, including large children's hospitals, children's hospitals within hospitals and general/community hospitals. Following an initial study group meeting this consortium agreed on a collective mission statement regarding paediatric procedural sedation. Decisions were based on guidelines from the American Academy of Paediatrics, American Society of Anaesthesiologists and American College of Emergency Physicians regarding sedation/anaesthesia of paediatric patients, a review of the literature and the consensus of the consortium members. Besides sharing a common mission on PSA, the PSRC is a data-sharing group: all participators agree to perform periodic audits of records to assure data and to maintain a prospective registry of all patients receiving PSA. ${ }^{28}$

Transient $\mathrm{O}_{2}$ desaturation below $90 \%$ for more than 30 seconds occurred 154 times per 10000 propofol administrations (1.5\%). Central apnea or airway obstruction occurred 575 times per 10000 administrations (5.8\%). Per 10000 encounters stridor occurred 50 times (0.5\%), laryngospasm 96 times (0.96\%), excessive secretions 341 times (3.4\%), and vomiting 49 times 
(0.49\%). Aspiration occurred 4 times during these 10000 sedation/anaesthesia encounters (0.04\%). There were no deaths. Cardiopulmonary resuscitation was required twice $(0.02 \%)$. The sedating professionals could manage all adverse events appropriately. In an unadjusted analysis, the rate of pulmonary adverse events was not different for anaesthesiologists versus other providers. Young age ( $<6$ months), fasting time $<8$ hours, ASA classification III or higher and concomitant use of opoids were all significantly related with a higher risk for respiratory adverse events. $^{42}$

\section{Adverse effects of Ketamine}

Several authors studied ketamine for PSA during oncology procedures (lumbar punctures, bone marrow punctures and/or bone biopsies), performed by non-anaesthesiologists. Evans et al. reported an incidence of desaturation of $1.7 \%$ and no airway obstruction during 119 sedation sessions ${ }^{43}$ Cheuk et al. reported an incidence of desaturations of $8.7 \%$ during 369 sedation sessions. These desaturations only required brief treatment with oxygen. No apneas or airway obstructions occurred. ${ }^{44}$ In a prospective study by Meyer et al. of 183 PSA sessions potentially serious complications were desaturation < $90 \%$ (5.4\%) and laryngospasm (0.5\%). ${ }^{45}$ Both intravenously (IV) and intramuscularly (IM) administered Ketamine was studied for PSA in painful ED procedures. In a recent meta-analysis of 8282 children receiving PSA with ketamine for procedures in an ED, the overall incidence of respiratory adverse events was 3.9\%. Independent risk factors were high intravenous doses, administration to children younger than 2 years or aged 13 years or older, and the concomitant use of anticholinergics or benzodiazepines. Variables without independent association included oropharyngeal procedures, underlying physical illness (American Society of Anaesthesiologists class >or $=3$ ), and the choice of intravenous versus intramuscular route. ${ }^{46}$ A retrospective analysis by Green et al. of a series of cases $(N=636)$ in which sedation with ketamine was administered by paediatric gastro-enterologists for gastrocopies in children, showed a high incidence of laryngospasm $(13.9 \%$ in the age group < 6 years; $3.6 \%$ in the age group $>6$ years). ${ }^{47}$

\section{Adverse effects of Dexmedetomidine}

In the last few years dexmedetomidine has been studied for PSA in children undergoing painless procedures. Regarding effectiveness for sedation in diagnostic imaging dexmedetomidine is significantly superior to midazolam and similar to propofol. ${ }^{48,49}$ Berkenbosch et al. published a prospective case series reporting the use of Dexmedetomidine in 48 children. Heart rate, blood pressure, and respiratory rate decreased but remained within normal limits for age. End-tidal $\mathrm{CO}_{2}$ exceeded $50 \mathrm{~mm} \mathrm{Hg}$ in seven of 404 measurements (1.7\%). ${ }^{50}$ Mason et al studied dexmedetomidine for sedation for computer tomography imaging (CT) in 62 patients. Heart rate (HR) and mean arterial blood pressure decreased an average of $15 \%$ and no significant respiratory changes were observed. ${ }^{51}$ In another study $(\mathrm{N}=250)$ these authors showed that individual titration of dexmedetomidine for $\mathrm{CT}$ imaging is associated with modest fluctuations in HR and blood pressure which were independent of age, required no pharmacologic interventions and did not result in any adverse events. ${ }^{52}$ In a prospective study by the same group dexmedetomidine as sole agent for paediatric MRI was studied in 747 consecutive patients. Three different dosing groups were analysed. Bradycardia without hypotension occurred in $16 \%$ of cases. There were no respiratory adverse events. ${ }^{53}$ Ray and Tobias retrospectively reviewed the charts of 42 children with autism pervasive developmental disorders and epilepsy, who received dexmedetomidine for sedation during electro encephalography. No significant hemodynamic or respiratory effects were noted. ${ }^{54}$ In two separate randomized controlled trials Koroglu et al. compared 
dexmedetomidine with respectively midazolam and propofol for sedation in children undergoing MRI scanning. No relevant adverse events were seen in the children sedated with dexmedetomidine ( $\mathrm{N}=70) .{ }^{48,49}$

\section{Adverse effects of Remifentanil}

Remifentanil, a potent ultra-short acting synthetic opioid, has been studied for PSA in children undergoing short painful procedures (e.g. lumbar puncture, bone marrow puncture), both as a sole agent and combined with Midazolam or Propofol. Litman et al. reported a high incidence of potentially life threatening respiratory depression in children undergoing painful procedures with the combination of a benzodiazepine and remifentanil. Out of 31 patients 25 (80.6\%) developed an apnea, requiring constant stimulation, and 10 (32.3\%) became hypoxemic ${ }^{55}$ Keidan et al. published a randomized controlled trial comparing propofol $(\mathrm{N}=36)$ and propofolremifentanil $(\mathrm{N}=41)$ for bone marrow aspiration in children. The addition of remifentanil was associated with a decrease in propofol dose and, consequently, recovery time, but with an increased risk of respiratory depression: hypoventilation or hypoxemia were significantly more frequent if remifentanil was added (19.5\% versus $11.1 \%){ }^{56}$ In a recent randomized controlled trial by Antmen et al. (A2) Eighty children undergoing bone marrow aspiration were randomly assigned to one of four sedation regimens: remifentanil $1 \mathrm{mcg} / \mathrm{kg}$ ( $\mathrm{N}=20)$, midazolam $0.05 \mathrm{mg} / \mathrm{kg}$ + remifentanil $0.5 \mathrm{mcg} / \mathrm{kg} / \mathrm{min}(\mathrm{N}=20)$, alfentanil $20 \mathrm{mcg} / \mathrm{kg}(\mathrm{N}=20)$ and midazolam $0.05 \mathrm{mg} / \mathrm{kg}+$ alfentanil $20 \mathrm{mcg} / \mathrm{kg}(\mathrm{N}=20)$. Relevant adverse events occurred in none of the 4 groups. ${ }^{57}$

\section{Adverse effects of Nitrous Oxide}

A French multicentric prospective study by Gall et al. of 7,511 sedation sessions with $50 \%$ nitrous oxide/ $50 \%$ oxygen premix, investigated the incidence of serious complications (oxygen desaturation, airway obstruction, apnea, bradycardia and/or oversedation). Such complications occurred in 25 sessions (0.3\%). In all cases, the problems dissolved instantly after discontinuation of the administration of nitrous oxide, without any need for airway intervention or ventilation. The main risk factors were age ( $<1$ year) and the simultaneous administration of benzodiazepines and opiates. ${ }^{58}$

Zier et al. reported a case series of 1018 sedation sessions using nurse-administered nitrous oxide (continuous flow; concentration of $70 \%$ ) for urinary catheterization. Only minor adverse events (diaphoresis, nausea and vomiting) were observed in $4 \%$ of the sessions. Oversedation without respiratory compromise occurred in $0.8 \%$ of cases. ${ }^{59}$ Babl et al. studied prospectively the relationship between fasting status and adverse events in 220 patients receiving nitrous oxide in a paediatric ED. Fasting status was obtained in 218 patients (99.1\%). Of these, 155 (71.1\%) did not meet fasting guidelines for solids. There were no serious adverse events and no episodes of aspiration. Emesis occurred in $7 \%$ of cases. There was no significant difference in median fasting time between patients with and without emesis. ${ }^{60}$

The same author studied prospectively the safety of high-concentration continuous-flow nitrous oxide $(70 \%$ versus $50 \%)$ in children $(\mathrm{N}=762$, age range $1-17$ yrs). Sixty-three (8.3\%) patients sustained mild and self-resolving adverse events, most of which were vomiting (5.7\%); 2 patients $(0.2 \%)$ had serious adverse events. Both serious events ( 1 chest pain and 1 desaturation) occurred in the group of $70 \%$ nitrous oxide. There was no significant difference in adverse events rates between nitrous oxide $70 \%(8.4 \%)$ and nitrous oxide $50 \%(9.9 \%){ }^{61}$ 


\subsection{Data from literature on the predictability and controllability of sedation depth}

It has been shown that unexpected deep sedation is associated with a higher rate of adverse events. ${ }^{13,21}$ The predictability of final sedation levels of a certain drug therefore determines the skills and competence the professionals in charge should possess. A search was made of existing literature on this subject. Results are summarized in table 3. 'Conclusions regarding predictability and controllability of non-titratable drugs intended for PSA'.

Table 3: Conclusions regarding predictability and controllability of non-titratbale drugs intended for PSA

\begin{tabular}{|c|c|c|}
\hline $\mathrm{Nr}$ & Conclusion & $\begin{array}{l}\text { Quality } \\
\text { Level }\end{array}$ \\
\hline \multirow[t]{2}{*}{1} & $\begin{array}{l}\text { For a PSA with medicines that are difficult to titrate and/or long-acting (e.g. chloral hydrate, } \\
\text { midazolam, barbiturates, opiates or combinations), the eventual depth of sedation, effective- } \\
\text { ness and duration of the sedation and timing of adverse events cannot reliably be predicted. } \\
\text { Therefore possible adverse effects of any possible sedation depth should always be anticipated } \\
\text { in terms of recognition and treatment. }\end{array}$ & Level 2 \\
\hline & B Hoffman $2002^{13}$, Newman $2003^{33}$, Malviya $2004^{62}$, Motas $2004^{21}$ & \\
\hline
\end{tabular}

Motas et al. published an observational study in 86 children who underwent PSA using midazolam, midazolam in combination with fentanyl or pethidine, chloral hydrate, pentobarbital or ketamine. Sedation depth was assessed by an independent observer, using a validated sedation scale and by bispectral cerebral function monitoring $\left(\mathrm{BIS}^{\circledast}\right)$. These observations were compared to the sedation depth the practitioners set out to achieve. The intended sedation depth was reached in $72 \%$ (sedation scale) and in $52 \%\left(\mathrm{BIS}^{\circledR}\right.$ ) of the cases respectively. In $35 \%$ of the cases, the $\mathrm{BIS}^{\circledast}$ figure present was consistent with general anaesthesia. The incidence of airway complications was significantly higher in the group that had been deeply sedated unintentionally. ${ }^{21}$

A risk assessment by Hoffman et al. based on prospective collected data of 96 sedations for widely varying procedures with chloral hydrate $(15 \%)$, midazolam $(28 \%)$, fentanyl (1\%), pentobarbital $(28 \%)$, ketamine $(2.8 \%)$ or cocktails of 3 or more of the medicines $(5.7 \%)$, showed that in $22 \%$ of the procedures, a deep sedation level was reached, although deep sedation had only been intended in $7 \%$ of the procedures. ${ }^{13}$

Malviya et al. studied two different types of discharge criteria in 29 children who had been sedated for an echocardiography $(27 / 29=93.1 \%$ with chloral hydrate and $2 / 29=6.9 \%$ with midazolam + diphenhydramine). Standard criteria (normal vital parameters, normal oxygen saturation, return to original consciousness level, normal cough and swallowing reflexes, normal movement) were compared with an objective assessment of the consciousness using BIS ${ }^{\circledR}$ monitoring and two validated scales of observation. The objective criteria correlated better with being fully awake than the standard criteria but it took significantly more time before those objective criteria were reached. ${ }^{62}$

The under 1.2 cited study by Newman et al. (prospective study of 1341 PSA sessions in children in an emergency department (ED)) showed an incidence of serious complications of $11.9 \%$ of which $92 \%$ occurred during the actual procedure, whereas the rest occurred after the procedure up to 40 minutes after the last administered dose of sedative. ${ }^{33}$ 


\section{Requisite skills and competences to guarantee optimal effectiveness}

Effectiveness is named as an outcome measure in most of the studies on PSA published over the last few decades. Mutual comparisons or combining averages is impossible, because the definition of effectiveness varies considerably for each procedure, or because it is not properly defined at all. No prospective controlled studies were found comparing different levels of professional skills/competence and the effectiveness of PSA. Evidence was therefore searched in an indirect way by looking for which PSA techniques a professional should master in order to achieve optimal PSA effectiveness. We defined that an optimal PSA technique should achieve near $100 \%$ predictable procedural success and timing, an optimal match between desired and achieved levels of sedation, minimal induction and recovery times and an optimal patient comfort by minimizing procedural pain, anxiety and the need for physical immobilization or restraint. Next we looked for settings and techniques with published evidence for contributing in reaching this optimal level. Results were classified as conclusions in four different categories of techniques or strategies with a proven effect on PSA effectiveness.

\subsection{Effect of the introduction of a dedicated well-trained team for PSA on the effectiveness of PSA}

Several authors have shown that the introduction of a dedicated PSA team that works according to published guidelines results in a significant decrease of procedural failure. (Level $\mathbf{2}$ conclusion based on Hoffman 2002 (B), Ruess 2002(C), Sury 1999(C)). ${ }^{13,15,63}$ Although it is impossible to deduce from those studies to what extent this result is due to specific professional skills and competence, PSA seems to become more effective when specifically trained professionals perform PSA in accordance with international guidelines.

\subsection{The superiority of titratable medicines or medicines with a highly predictable effectiveness, including deep sedation}

In order to achieve an optimal level of effectiveness, each PSA should ideally be directed to an individually determined sedation level. This makes the use of short acting drugs (e.g. propofol) that can be titrated to the desired level of sedation (including deep sedation) advantageous over the use of long acting drugs. There is growing evidence for the need for deep sedation for the majority of procedures in paediatrics. A retrospective analysis by Dial et al. of the sedation depth that was eventually required for a category of examinations $(N=32)$ that were not (very) painful and for which immobility was not strictly required turned out to be deep sedation after all in $26 / 32$ cases (81.3\%). For the category of painful and invasive examinations for which local anaesthesia was used light to moderate sedation turned out to be sufficient in only 4/156 cases (2.6\%), whereas deep sedation was necessary in $136 / 156$ cases (87.2\%) and even a general anaesthesia in $16 / 156$ cases $(10.3 \%) .{ }^{24}$. On the other hand there is good evidence for the superior effectiveness of PSA with titratable medicines with a clearly predictable effectiveness. This has been demonstrated in children undergoing very painful procedures (e.g. oncological procedures, procedures in an ED), (protracted) stressful procedures (e.g. endoscopies) and procedures for which patients need to lie still for long periods (e.g. for imaging and radiotherapy). In addition, working with propofol also leads to a significantly shorter induction time and a significantly quicker recovery. Having the requisite competencies and skills to use this sort of sedatives safely therefore seems important to guarantee optimal effectiveness (Level 1 conclusion based on Migita 2006 (A1), Marx 1997 (A2), Pershad 2007 (A2), Dalal 2006(B), Seiler 2001(B), lannalfi 2005(B), Kohsoo 2003(B), Holdsworth 2003(B)) ${ }^{64-70}$ 


\subsection{Deployability of techniques for light sedation}

Children often have to be physically forced or restrained for so-called 'minor painful procedures' (e.g. (blood sampling, inserting an intravenous access, suturing a wound, lumbar puncture, bone marrow puncture, changing a dressing, incision of abscess, resection of naevus or cyst, bladder catheterization, intra-articular injection and Ear Nose Throat procedures). It has been demonstrated that the level of comfort during such interventions can be considerably improved when nitrous oxide is used. Nitrous oxide in concentrations of up to $70 \%$, when combined with nonpharmacological distraction techniques and adequate topical anaesthesia, is a very effective and safe way to suppress procedural pain and stress in children $>1$ year old. (Level $\mathbf{3}$ conclusion based on lannalfi 2005(B), Kanagasundaram 2001(C), Burnweit 2004(C), Frampton 2003(C), Zier 2007 (C). ${ }^{59,71-74}$ For children undergoing reduction of an uncomplicated forearm reduction nitrous oxide in concentrations of $50 \%$ in combination with local anaesthetics is equally effective as intravenous ketamine but is associated with a significantly shorter recovery time and less respiratory side effects. (Level 3 conclusion based on Luhmann $2006(B)) .{ }^{75}$ In children that need to receive sutures, nitrous oxide in concentrations of $50 \%$ in combination with local anaesthetics controls the procedural pain and stress more effectively than orally taken midazolam or local anaesthetics alone. (Level 2 conclusion based on Luhmann 2001(B), Bar-Meir 2006(B)). ${ }^{76,77}$ Inserting a venous access in children who are known to have difficult veins is easier under sedation with nitrous oxide + topical anaesthesia than topical anaesthesia alone. (Level 3; Ekbom 2005(B)). ${ }^{78}$ Topical anaesthesia and nitrous oxide combined are more effective than topical anaesthesia or nitrous oxide alone. (Level 1 conclusion based on Paut 2001(A2), Hee 2003(A2)). 79,80

\subsection{Use of non-pharmacological techniques}

In literature good evidence is available for the importance of applying non-pharmacological techniques to improve procedural success and comfort. When a professional takes care over providing good information about the procedure to be followed, this may result in the children feeling less stress during the procedure and being less scared about future procedures. (Level 3 conclusion based on Lewis-Claar 2002(C), Bishop 2002(C)). ${ }^{81,82}$ Adequate information also helps parents to provide better support for their children during a painful procedure. (Level 3 conclusion based on Kupietzky 2002(C), Cline 2006(D)) ${ }^{83,84}$ A child (> 4 years old) that receives sufficient preparation (e.g. by information, practice, simulation, play therapy) before an MRI examination, a gastroscopy or nuclear examination will experience less distress during the procedure and will require less sedation or analgesia. (Level 2 conclusion based on Mahajan 1998 (A2), Rosenberg 1997(B), Presdee 1997(C), Awogbemi 2005 C), de Amorim e Silva 2006 C)) ${ }^{85-89} \mathrm{Be}-$ tween 1993 and 20093 high-quality Systematic Reviews (SR) of nonpharmacological interventions for procedure related pain in children have been published, allowing 3 Level 1 conclusions. Cepeda et al. (2006;A1 including 51 RCT's of which only 4 addressed procedural pain in children) could not demonstrate evidence for the effectiveness of music therapy during intravenous cannulation and vaccination in children. Although listening to music reduced pain intensity scales in general and opioid requirements in particular, the reported effects are small. Pooling of the 4 studies was impossible due to different quantification methods of pain intensity ${ }^{90}$ Richardson et al. published a SR (2006; A1 including 7 RCTs and 1 non-RCT) on the pain reducing effects of hypnosis in paediatric cancer patients undergoing common painful procedures (infusapost access, venipuncture, lumbar puncture and bone marrow aspiration). Although $7 / 8$ studies included reported a significant reduction of pain, the authors conclude that due to methodological limitations there is no conclusive evidence for a significant effect of hypnosis on procedure 
related pain. ${ }^{91}$ Finally, the SR by Uman et al (2006;A1 including 28 trials) showed a significant effect of distraction and hypnosis on self-reported pain during needle-related procedures (intramuscular injection, vaccination, venipuncture, intravenous cannulation, lumbar punction and bone marrow aspiration). For other psychologhical techniques no significant effect on procedure-related pain could be concluded. ${ }^{92}$

An additional SR by Kleiber and Harper (1999) focused on the effects of distraction on selfreported pain in children during intravenous cannulation, lumbar punction, bone marrow aspiration, injection, venipuncture, dental procedures and burn treatment. They showed that distraction causes a significant reduction of self-reported pain. An important limitation of this SR is the fact that no details are provided on the methodological quality of the included studies. ${ }^{93}$ None of the SR could demonstrate any adverse events of non-pharmacological techniques.

Hypnosis on children reduces procedure-related pain and distress more effectively compared to local anaesthesia (venipuncture and lumbar puncture; Level 1 conclusion based on three independent A2 studies by the same authors: Liossi 2009, Liossi 2006 and Liossi 2003), to cognitive behavioral therapy or no therapy (bone marrow aspiration; Level 2 conclusion based on Liossi 1999 (A2)) and to standard medical care including relaxation exercises or play intervention (cystogram; Level 2 conclusion based on Butler 2005(A2)). ${ }^{94-98}$

In conclusion we found that a professional able to use psychological techniques for distraction or hypnosis during painful and/or stressful medical procedures may be able to reduce the child's procedural distress. Furthermore, the use of psychological techniques intended to distract children during a painful and/or stressful medical procedure reduces the need for sedation (Level 2 conclusion based on Harned 2001(B) and Train 2006(B)) ${ }^{99,100}$

\section{Discussion}

This review shows sufficient evidence to support the statement that safety and effectiveness of PSA are significantly related to the level of professional skills and competence. Although there are no prospective studies comparing the effect of different levels of skills and competence on PSA related safety and effectiveness, this systematic review identified in the relevant literature which competences and skills a professional should possess or achieve in order to be able to perform PSA in children safely and effectively. For that purpose we systematically summarized the results in conclusions classified according to the strength of evidence of the contributing papers. These conclusions can be translated into recommendations on the general skills and competence any professional entrusted with PSA must have in order to achieve optimal safety and effectiveness. (Table 4 and 5) 
Table 4: General recommendations on necessary skills and competence for achieving optimal PSA related safety and effectiveness in children

\begin{tabular}{ll}
\hline $\mathbf{N r}$ & Recommendations \\
\hline 1 & $\begin{array}{l}\text { Knowledge of the drug dosing, dosing techniques, indications, contra-indications and requisite precautions } \\
\text { of the sedation technique used, acquired through specific training or demonstrable relevant experience. }\end{array}$ \\
2 & Regular personal experience of the applied medication or technique*. \\
3 & $\begin{array}{l}\text { Applying the form of sedation that is most appropriate for the procedure and the patient. This implicates the } \\
\text { ability to guarantee the optimally effective sedation level in a predictable manner. An optimal PSA technique } \\
\text { should achieve near } 100 \% \text { predictable procedural success and timing, an optimal match between desired } \\
\text { and achieved levels of sedation, minimal induction and recovery times and an optimal patient comfort by } \\
\text { minimizing procedural pain, anxiety and the need for physical immobilization or restraint. }\end{array}$
\end{tabular}

4 The ability to perform pre-procedural screening and a systematic risk analysis.

5 The ability to inform the patient, parents or carers about the sedation technique, the effects, potential side effects and possible alternatives. The information must be given in time and be appropriate for the comprehension level of the patient and parents/carers.

6 The ability to guarantee a child-centered approach within a general policy that favors children before, during and after the procedure.

7 The ability to apply, or arrange for complementary non-pharmacological techniques like preparation, distraction, combined cognitive-behavioral interventions and hypnosis.

8 The ability to (a) apply effective local or topical anaesthesia, if appropriate, and (b) to recognize and intervene with possible toxicity of local anaesthetic agents.

9 Organizing the necessary monitoring and rescue facilities during and after the procedure for as long as the consciousness level is lowered.

10 The ability to organize a supervised recovery phase and to define the discharge criteria.

11 The ability to organize the prompt availability of a resuscitation team or a professional trained in Paediatric Life Support.

12 Supervising, registering, assessing and optimizing the quality of the sedation in terms of safety and effectiveness.

\footnotetext{
* It is impossible to derive from literature a more precise definition of "regular personal experience ". The authors believe that regular experience means a minimal of 50 PSA sessions per year
}

Besides general recommendations we formulated additional recommendations depending on the level of sedation. Contrary to the generally accepted division between mild, moderate and deep sedation in most guidelines we believe that, based on the evidence, having different levels of monitoring and competence for moderate and deep sedation is arbitrary and potentially dangerous. Ever since the first guideline on PSA was published, authors have linked the level of sedation with potential respiratory and cardiovascular side effects and by this with necessary safety precautions, monitoring and professional skills and competence. ${ }^{10}$ Consequently, definitions were made for light sedation, moderate sedation (formerly called 'conscious sedation'), deep sedation and anaesthesia. Light sedation, formerly called 'anxiolysis', is typically the result of one standard dose of midazolam or by the breathing of nitrous oxide (inspired concentration up to $50 \%) .{ }^{61}$ Higher doses, or other drugs, either alone or in combination, are likely to cause deeper levels of sedation. Commonly used PSA drugs intended for moderate sedation such as chloral hydrate, barbiturates, benzodiazepines with/without opioids and solely opioids cause wide variations in depth of sedation. If a single dose is given the goal of moderate sedation is not achieved or exceeded in a substantial number of children. Therefore, for individual cases, predic- 
tion of the effective sedation end point is unreliable. ${ }^{21}$ Multiple doses or combinations of drugs are more likely to cause deep sedation and are associated with hypoventilation, respiratory depression and serious morbidity. Considering sedation levels as a sliding scale, rather than a step-by-step change in consciousness, the transition from one level to another can be subtle and sudden. It is, therefore, advisable to recommend the same safety precautions and professional skills for all levels of sedation beyond light sedation, irrespective of the drug used for PSA. Consequently, it is wise to formulate separate recommendations regarding professional skills and competence for light sedation on one hand and for moderate to deep sedation on the other hand. (Table $5 a+5 b$ ) Although the safety profiles of PSA drugs are clearly different, the likelihood that potentially serious adverse events may happen and the predictability of depth and duration of sedation are clearly more important. Both issues have a direct impact on the imperative skills and competence, mainly in terms of timely recognition and appropriate management of possible adverse events. PSA related safety is determined by logistics, organization and professional skills rather than by specific pharmacologic characteristics.

Table 5a: Recommended specific additional skills and competence for achieving optimal safety during moderate and deep sedation in children

\begin{tabular}{ll}
\hline $\mathrm{Nr}$ & Recommendations \\
\hline 1 & In order to guarantee optimal levels of safety and effectiveness during a PSA involving (a possibility of) \\
moderate to deep sedation, the PSA must be carried out by a separate professional that is not involved in \\
the actual procedure. \\
During a PSA involving (a possibility of) moderate or deep sedation and during the subsequent recovery \\
phase, a professional must be present with at least the following additional competence and skills: \\
The ability to assess and interpret the sedation depth \\
The ability to guarantee the necessary monitoring of vital parameters, including capnography, and being \\
able to appraise and interpret the monitored information, \\
Having acquired the necessary knowledge during a specialist course and by means of refresher courses and \\
ability to manage the following techniques at APLS* level: \\
Techniques intended to guarantee an open airway, including skills to manage larynx spasm and to use \\
Laryngeal Mask Airways \\
Techniques to administer mask/bag ventilation. \\
The use of antagonists \\
Heart massage techniques \\
*APLS: Advanced Paediatric Life Support
\end{tabular}

Table 5b: Specific additional skills and competence for achieving optimal safety during light sedation/anxiolysis in children

\begin{tabular}{ll}
\hline $\mathbf{N r} \quad$ Recommendations \\
\hline $1 \quad$ During a PSA involving light sedation and during the subsequent recovery phase, a professional must be \\
present with the at least the following additional competence and skills: \\
The ability to assess and interpret the sedation depth \\
The ability to maintain continuous verbal contact with the patient in the absence of any other form of \\
monitoring. \\
Having acquired the necessary knowledge through a specialist course and by means of refresher courses and \\
the ability to manage the following techniques at BLS* level: \\
Techniques intended to guarantee an open airway \\
Techniques to administer mask/bag ventilation. \\
*BLS: Basic Life Support
\end{tabular}


In order to achieve an optimal level of effectiveness, each PSA should ideally be directed to an individually determined sedation level. This makes the use of short acting "titratable" drugs advantageous over the use of long acting drugs. Short acting drugs can be used to overcome the pain and distress that varies according to the procedures and the patients themselves. It can be concluded from this systematic review that professionals having the requisite skills and competence to work with titratable anaesthetics (e.g. propofol) are able to achieve more optimally an effective PSA for children undergoing very painful procedures (e.g. oncological procedures, procedures in an ED), (protracted) stressful procedures (e.g. endoscopies) and procedures for which patients need to lie still for long periods (e.g. diagnostic imaging and radiotherapy). In particular young children ( $<6$ years) are in need of deep sedation sometimes even for so called "mild" procedures. ${ }^{23,24}$ Although the obvious advantages of titratable deep sedation (e.g. using propofol) over other sedatives for many procedures in children are increasingly emphasized in recent literature, the term deep sedation has been under discussion, because it may be indistinguishable from general anaesthesia. While this point may be overstated it has led to the widespread recommendation that the same personnel, equipment and facilities must be available to manage both deep sedation and anaesthesia. The most important severe adverse effect of propofol is respiratory depression, which is associated with unexpected deep sedation and can arise suddenly and unexpectedly. ${ }^{1}$ As a consequence the question whether non-anaesthesiologists can be safely entrusted with the use of this potent drug has been a matter of debate. ${ }^{23}$ There is an obvious reluctance by the anaesthetic world to entrust trained non-anaesthesiologist with highly active anaesthetic drugs. ${ }^{23,101}$ However, in many countries a clear trend is seen to entrust deep sedation to specifically trained non-anaesthesia professionals in particular because of the scarcity of anaesthesiologists. Emergency physicians, intensivists and gastro-enterologists have been prominent in this development. ${ }^{12,36,37,41,101,102}$ In addition, It has been shown that in optimal safety and monitoring conditions deep sedation using propofol is equally safe irrespective whether it is administered by trained non-anaesthesiologists or anaesthesiologists. ${ }^{42,}{ }^{101} \mathrm{An}$ evidence-based clinical practice advisory for the administration of propofol for PSA by nonanaesthesiologists was recently published. ${ }^{103}$

For minor painful procedures the deployability of short-acting light sedation using nitrous oxide and ability to apply adequate topical anaesthesia are essential skills for optimal effectiveness. In addition, not only the ability to define and apply an individually tailored PSA technique but also the ability to implement non-pharmacological techniques, such as distraction, hypnosis and combined cognitive-behavioral interventions, belongs to the essential competence and skills.

Finally we found evidence that the application of published guidelines within a well organized, well trained and dedicated PSA team will enhance PSA related safety and effectiveness.

In conclusion, PSA has to be considered as a separate medical act, provided by well-trained, competent and skilled professionals only, working within a context of transparency, registration and ongoing quality control. Skills and competence, rather than professional title, are determinants for safe and effective PSA. We believe that these evidence based recommendations regarding necessary skills and competence should be used to set up training programs and to define which professionals can and cannot be credentialed for PSA in children. Much emphasis is needed for adequate and effective implementation strategies for these recommendations. 


\section{References}

1. Krauss B, Green SM. Procedural sedation and analgesia in children. Lancet. 2006;367(9512):766-780.

2. Cote CJ. Discharge criteria for children sedated by nonanaesthesiologists: is "safe" really safe enough? Anaesthesiology. 2004;100(2):207-209.

3. Ratnapalan S, Schneeweiss S. Guidelines to practice: the process of planning and implementing a paediatric sedation program. Paediatric emergency care. 2007;23(4):262-266.

4. Cote CJ, Karl HW, Notterman DA, Weinberg JA, McCloskey C. Adverse sedation events in paediatrics: analysis of medications used for sedation. Paediatrics. 2000;106(4):633-644.

5. Cote CJ, Notterman DA, Karl HW, Weinberg JA, McCloskey C. Adverse sedation events in paediatrics: a critical incident analysis of contributing factors. Paediatrics. 2000;105(4 Pt 1):805-814.

6. Guidelines for monitoring and management of paediatric patients during and after sedation for diagnostic and therapeutic procedures: addendum. Paediatrics. 2002;110(4):836-838.

7. Practice guidelines for sedation and analgesia by non-anaesthesiologists. Anaesthesiology. 2002;96(4): 1004-1017.

8. Yldzdas D, Yapcoglu H, Ylmaz HL. The value of capnography during sedation or sedation/analgesia in paediatric minor procedures. Paediatric emergency care. 2004;20(3):162-165.

9. Guideline statement: management of procedure-related pain in children and adolescents. $J$ Paediatr Child Health. 2006;42 Suppl 1:S1-29.

10. Cote CJ, Wilson S. Guidelines for monitoring and management of paediatric patients during and after sedation for diagnostic and therapeutic procedures: an update. Paediatrics. 2006;118(6):2587-2602.

11. Polaner DM, Houck CS, Rockoff MA, Mancuso TJ, Finley GA, Maxwell LG, et al. Sedation, risk, and safety: do we really have data at last? Paediatrics. 2001;108(4):1006-1008.

12. Barbi E, Gerarduzzi T, Marchetti F, Neri E, Verucci E, Bruno I, et al. Deep sedation with propofol by nonanaesthesiologists: a prospective paediatric experience. Archives of paediatrics \& adolescent medicine. 2003;157(11):1097-1103.

13. Hoffman GM, Nowakowski R, Troshynski TJ, Berens RJ, Weisman SJ. Risk reduction in paediatric procedural sedation by application of an American Academy of Paediatrics/American Society of Anaesthesiologists process model. Paediatrics. 2002;109(2):236-243.

14. Pitetti R, Davis PJ, Redlinger R, White J, Wiener E, Calhoun KH. Effect on hospital-wide sedation practices after implementation of the $2001 \mathrm{JCAHO}$ procedural sedation and analgesia guidelines. Archives of paediatrics \& adolescent medicine. 2006;160(2):211-216.

15. Ruess L, O'Connor SC, Mikita CP, Creamer KM. Sedation for paediatric diagnostic imaging: use of paediatric and nursing resources as an alternative to a radiology department sedation team. Paediatric radiology. 2002;32(7):505-510.

16. Babl FE, Munro J, Kainey G, Palmer GM, Iser A. Scope for improvement: hospital wide sedation practice at a children's hospital. Archives of disease in childhood. 2006;91(8):716-717.

17. Leroy P, Nieman F, Blokland-Loggers H, Schipper D, Zimmermann L, Knape H. Adherence to safety guidelines on paediatric procedural sedation: the results of a nationwide survey under general paediatricians in The Netherlands. Archives of disease in childhood. 2009 [Epub ahead of Print].

18. Cravero JP, Blike GT. Review of paediatric sedation. Anaesthesia and analgesia. 2004;99(5):1355-1364.

19. Leroy PL, Gorzeman MP, Sury MR. Procedural sedation and analgesia in children by nonanaesthesiologists in an emergency department. Minerva paediatrica. 2009;61(2):193-215.

20. Malviya S, Voepel-Lewis T, Eldevik OP, Rockwell DT, Wong JH, Tait AR. Sedation and general anaesthesia in children undergoing MRI and CT: adverse events and outcomes. British journal of anaesthesia. 2000;84(6):743-748.

21. Motas D, McDermott NB, VanSickle T, Friesen RH. Depth of consciousness and deep sedation attained in children as administered by nonanaesthesiologists in a children's hospital. Paediatric anaesthesia. 2004;14(3):256-260.

22. SIGN Guideline 58: Safe sedation of children undergoing diagnostic and therapeutic procedures http://www.sign.ac.uk/pdf/sign58.pdf. Published 2004. 
23. Cote CJ. Round and round we go: sedation -- what is it, who does it, and have we made things safer for children? Paediatric anaesthesia. 2008;18(1):3-8.

24. Dial S, Silver P, Bock K, Sagy M. Paediatric sedation for procedures titrated to a desired degree of immobility results in unpredictable depth of sedation. Paediatric emergency care. 2001;17(6):414-420.

25. Folkes K. Is restraint a form of abuse? Paediatric nursing. 2005;17(6):41-44.

26. Sanborn PA, Michna E, Zurakowski D, Burrows PE, Fontaine PJ, Connor L, et al. Adverse cardiovascular and respiratory events during sedation of paediatric patients for imaging examinations. Radiology. 2005;237(1):288-294.

27. Malviya S, Voepel-Lewis T, Tait AR. Adverse events and risk factors associated with the sedation of children by nonanaesthesiologists. Anaesthesia and analgesia. 1997;85(6):1207-1213.

28. Cravero JP, Blike GT, Beach M, Gallagher SM, Hertzog JH, Havidich JE, et al. Incidence and nature of adverse events during paediatric sedation/anaesthesia for procedures outside the operating room: report from the Paediatric Sedation Research Consortium. Paediatrics. 2006;118(3):1087-1096.

29. Mason KP, Sanborn P, Zurakowski D, Karian VE, Connor L, Fontaine PJ, et al. Superiority of pentobarbital versus chloral hydrate for sedation in infants during imaging. Radiology. 2004;230(2):537-542.

30. Mason KP, Zurakowski D, Connor L, Karian VE, Fontaine PJ, Sanborn PA, et al. Infant sedation for MR imaging and CT: oral versus intravenous pentobarbital. Radiology. 2004;233(3):723-728.

31. Mason KP, Zurakowski D, Karian VE, Connor L, Fontaine PJ, Burrows PE. Sedatives used in paediatric imaging: comparison of IV pentobarbital with IV pentobarbital with midazolam added. Ajr. 2001;177(2):427-430.

32. Roback MG, Wathen JE, Bajaj L, Bothner JP. Adverse events associated with procedural sedation and analgesia in a paediatric emergency department: a comparison of common parenteral drugs. Acad Emerg Med. 2005;12(6):508-513.

33. Newman DH, Azer MM, Pitetti RD, Singh S. When is a patient safe for discharge after procedural sedation? The timing of adverse effect events in 1367 paediatric procedural sedations. Annals of emergency medicine. 2003;42(5):627-635.

34. Pena BM, Krauss B. Adverse events of procedural sedation and analgesia in a paediatric emergency department. Annals of emergency medicine. 1999;34(4 Pt 1):483-491.

35. Pitetti RD, Singh S, Pierce MC. Safe and efficacious use of procedural sedation and analgesia by nonanaesthesiologists in a paediatric emergency department. Archives of paediatrics \& adolescent medicine. 2003;157(11):1090-1096.

36. Hertzog JH, Campbell JK, Dalton HJ, Hauser GJ. Propofol anaesthesia for invasive procedures in ambulatory and hospitalized children: experience in the paediatric intensive care unit. Paediatrics. 1999;103(3):E30.

37. Hertzog JH, Dalton HJ, Anderson BD, Shad AT, Gootenberg JE, Hauser GJ. Prospective evaluation of propofol anaesthesia in the paediatric intensive care unit for elective oncology procedures in ambulatory and hospitalized children. Paediatrics. 2000;106(4):742-747.

38. Pershad J, Godambe SA. Propofol for procedural sedation in the paediatric emergency department. The Journal of emergency medicine. 2004;27(1):11-14.

39. Bassett KE, Anderson JL, Pribble CG, Guenther E. Propofol for procedural sedation in children in the emergency department. Annals of emergency medicine. 2003;42(6):773-782.

40. Guenther E, Pribble CG, Junkins EP, Jr., Kadish HA, Bassett KE, Nelson DS. Propofol sedation by emergency physicians for elective paediatric outpatient procedures. Annals of emergency medicine. 2003;42(6):783-791.

41. Vespasiano M, Finkelstein M, Kurachek S. Propofol sedation: intensivists' experience with 7304 cases in a children's hospital. Paediatrics. 2007;120(6):e1411-1417.

42. Cravero JP, Beach ML, Blike GT, Gallagher SM, Hertzog JH. The incidence and nature of adverse events during paediatric sedation/anaesthesia with propofol for procedures outside the operating room: a report from the Paediatric Sedation Research Consortium. Anaesthesia and analgesia. 2009;108(3):795804.

43. Evans D, Turnham L, Barbour K, Kobe J, Wilson L, Vandebeek C, et al. Intravenous ketamine sedation for painful oncology procedures. Paediatric anaesthesia. 2005;15(2):131-138. 
44. Cheuk DK, Wong WH, Ma E, Lee TL, Ha SY, Lau YL, et al. Use of midazolam and ketamine as sedation for children undergoing minor operative procedures. Support Care Cancer. 2005;13(12):1001-1009.

45. Meyer S, Aliani S, Graf N, Reinhard H, Gottschling S. Sedation with midazolam and ketamine for invasive procedures in children with malignancies and hematological disorders: a prospective study with reference to the sympathomimetic properties of ketamine. Paediatric hematology and oncology. 2003;20(4):291-301.

46. Green SM, Roback MG, Krauss B, Brown L, McGlone RG, Agrawal D, et al. Predictors of airway and respiratory adverse events with ketamine sedation in the emergency department: an individual-patient data meta-analysis of 8,282 children. Annals of emergency medicine. 2009;54(2):158-168 e151-154.

47. Green SM, Klooster M, Harris T, Lynch EL, Rothrock SG. Ketamine sedation for paediatric gastroenterology procedures. Journal of paediatric gastroenterology and nutrition. 2001;32(1):26-33.

48. Koroglu A, Demirbilek S, Teksan H, Sagir O, But AK, Ersoy MO. Sedative, haemodynamic and respiratory effects of dexmedetomidine in children undergoing magnetic resonance imaging examination: preliminary results. British journal of anaesthesia. 2005;94(6):821-824.

49. Koroglu A, Teksan H, Sagir O, Yucel A, Toprak HI, Ersoy OM. A comparison of the sedative, hemodynamic, and respiratory effects of dexmedetomidine and propofol in children undergoing magnetic resonance imaging. Anaesthesia and analgesia. 2006;103(1):63-67, table of contents.

50. Berkenbosch JW, Wankum PC, Tobias JD. Prospective evaluation of dexmedetomidine for noninvasive procedural sedation in children. Paediatr Crit Care Med. 2005;6(4):435-439; quiz 440.

51. Mason KP, Zgleszewski SE, Dearden JL, Dumont RS, Pirich MA, Stark CD, et al. Dexmedetomidine for paediatric sedation for computed tomography imaging studies. Anaesthesia and analgesia. 2006;103(1):57-62, table of contents.

52. Mason KP, Zgleszewski SE, Prescilla R, Fontaine PJ, Zurakowski D. Hemodynamic effects of dexmedetomidine sedation for CT imaging studies. Paediatric anaesthesia. 2008;18(5):393-402.

53. Mason KP, Zurakowski D, Zgleszewski SE, Robson CD, Carrier M, Hickey PR, et al. High dose dexmedetomidine as the sole sedative for paediatric MRI. Paediatric anaesthesia. 2008;18(5):403-411.

54. Ray T, Tobias JD. Dexmedetomidine for sedation during electroencephalographic analysis in children with autism, pervasive developmental disorders, and seizure disorders. Journal of clinical anaesthesia. 2008;20(5):364-368.

55. Litman RS. Conscious sedation with remifentanil during painful medical procedures. Journal of pain and symptom management. 2000;19(6):468-471.

56. Keidan I, Berkenstadt H, Sidi A, Perel A. Propofol/remifentanil versus propofol alone for bone marrow aspiration in paediatric haemato-oncological patients. Paediatric anaesthesia. 2001;11(3):297-301.

57. Antmen B, Sasmaz I, Birbicer H, Ozbek H, Burgut R, Isik G, et al. Safe and effective sedation and analgesia for bone marrow aspiration procedures in children with alfentanil, remifentanil and combinations with midazolam. Paediatric anaesthesia. 2005;15(3):214-219.

58. Gall O, Annequin D, Benoit G, Glabeke E, Vrancea F, Murat I. Adverse events of premixed nitrous oxide and oxygen for procedural sedation in children. Lancet. 2001;358(9292):1514-1515.

59. Zier JL, Drake GJ, McCormick PC, Clinch KM, Cornfield DN. Case-series of nurse-administered nitrous oxide for urinary catheterization in children. Anaesthesia and analgesia. 2007;104(4):876-879.

60. Babl FE, Puspitadewi A, Barnett P, Oakley E, Spicer M. Preprocedural fasting state and adverse events in children receiving nitrous oxide for procedural sedation and analgesia. Paediatric emergency care. 2005;21(11):736-743.

61. Babl FE, Oakley E, Seaman C, Barnett P, Sharwood LN. High-concentration nitrous oxide for procedural sedation in children: adverse events and depth of sedation. Paediatrics. 2008;121(3):e528-532.

62. Malviya S, Voepel-Lewis T, Ludomirsky A, Marshall J, Tait AR. Can we improve the assessment of discharge readiness?: A comparative study of observational and objective measures of depth of sedation in children. Anaesthesiology. 2004;100(2):218-224.

63. Sury MR, Hatch DJ, Deeley T, Dicks-Mireaux C, Chong WK. Development of a nurse-led sedation service for paediatric magnetic resonance imaging. Lancet. 1999;353(9165):1667-1671. 
64. Dalal PG, Murray D, Cox T, McAllister J, Snider R. Sedation and anaesthesia protocols used for magnetic resonance imaging studies in infants: provider and pharmacologic considerations. Anaesthesia and analgesia. 2006;103(4):863-868.

65. Holdsworth MT, Raisch DW, Winter SS, Frost JD, Moro MA, Doran NH, et al. Pain and distress from bone marrow aspirations and lumbar punctures. The Annals of pharmacotherapy. 2003;37(1):17-22.

66. Khoshoo V, Thoppil D, Landry L, Brown S, Ross G. Propofol versus midazolam plus meperidine for sedation during ambulatory esophagogastroduodenoscopy. Journal of paediatric gastroenterology and nutrition. 2003;37(2):146-149.

67. Marx CM, Stein J, Tyler MK, Nieder ML, Shurin SB, Blumer JL. Ketamine-midazolam versus meperidinemidazolam for painful procedures in paediatric oncology patients. J Clin Oncol. 1997;15(1):94-102.

68. Migita RT, Klein EJ, Garrison MM. Sedation and analgesia for paediatric fracture reduction in the emergency department: a systematic review. Archives of paediatrics \& adolescent medicine. 2006;160(1):4651.

69. Pershad J, Wan J, Anghelescu DL. Comparison of propofol with pentobarbital/midazolam/fentanyl sedation for magnetic resonance imaging of the brain in children. Paediatrics. 2007;120(3):e629-636.

70. Seiler G, De Vol E, Khafaga Y, Gregory B, Al-Shabanah M, Valmores A, et al. Evaluation of the safety and efficacy of repeated sedations for the radiotherapy of young children with cancer: a prospective study of 1033 consecutive sedations. International journal of radiation oncology, biology, physics. 2001;49(3): 771-783.

71. Burnweit C, Diana-Zerpa JA, Nahmad MH, Lankau CA, Weinberger M, Malvezzi L, et al. Nitrous oxide analgesia for minor paediatric surgical procedures: an effective alternative to conscious sedation? Journal of paediatric surgery. 2004;39(3):495-499; discussion 495-499.

72. Frampton A, Browne GJ, Lam LT, Cooper MG, Lane LG. Nurse administered relative analgesia using high concentration nitrous oxide to facilitate minor procedures in children in an emergency department. Emerg Med J. 2003;20(5):410-413.

73. Iannalfi A, Bernini G, Caprilli S, Lippi A, Tucci F, Messeri A. Painful procedures in children with cancer: comparison of moderate sedation and general anaesthesia for lumbar puncture and bone marrow aspiration. Paediatric blood \& cancer. 2005;45(7):933-938.

74. Kanagasundaram SA, Lane $\amalg$, Cavalletto BP, Keneally JP, Cooper MG. Efficacy and safety of nitrous oxide in alleviating pain and anxiety during painful procedures. Archives of disease in childhood. 2001;84(6):492-495.

75. Luhmann JD, Schootman M, Luhmann SJ, Kennedy RM. A randomized comparison of nitrous oxide plus hematoma block versus ketamine plus midazolam for emergency department forearm fracture reduction in children. Paediatrics. 2006;118(4):e1078-1086.

76. Bar-Meir E, Zaslansky R, Regev E, Keidan I, Orenstein A, Winkler E. Nitrous oxide administered by the plastic surgeon for repair of facial lacerations in children in the emergency room. Plastic and reconstructive surgery. 2006;117(5):1571-1575.

77. Luhmann JD, Kennedy RM, Porter FL, Miller JP, Jaffe DM. A randomized clinical trial of continuous-flow nitrous oxide and midazolam for sedation of young children during laceration repair. Annals of emergency medicine. 2001;37(1):20-27.

78. Ekbom K, Jakobsson J, Marcus C. Nitrous oxide inhalation is a safe and effective way to facilitate procedures in paediatric outpatient departments. Archives of disease in childhood. 2005;90(10):1073-1076.

79. Hee HI, Goy RW, Ng AS. Effective reduction of anxiety and pain during venous cannulation in children: a comparison of analgesic efficacy conferred by nitrous oxide, EMLA and combination. Paediatric anaesthesia. 2003;13(3):210-216.

80. Paut O, Calmejane C, Delorme J, Lacroix F, Camboulives J. EMLA versus nitrous oxide for venous cannulation in children. Anaesthesia and analgesia. 2001;93(3):590-593.

81. Bishop PR, Nowicki MJ, May WL, Elkin D, Parker PH. Unsedated upper endoscopy in children. Gastrointestinal endoscopy. 2002;55(6):624-630.

82. Lewis Claar R, Walker LS, Barnard JA. Children's knowledge, anticipatory anxiety, procedural distress, and recall of esophagogastroduodenoscopy. Journal of paediatric gastroenterology and nutrition. 2002; $34(1): 68-72$. 
83. Cline RJ, Harper FW, Penner LA, Peterson AM, Taub JW, Albrecht TL. Parent communication and child pain and distress during painful paediatric cancer treatments. Social science \& medicine (1982). 2006;63(4):883-898.

84. Kupietzky A, Ram D. Effects of a positive verbal presentation on parental acceptance of passive medical stabilization for the dental treatment of young children. Paediatric dentistry. 2005;27(5):380-384.

85. Awogbemi T, Watson AR, Hiley D, Clarke L. Preparing children for day case nuclear medicine procedures. Nuclear medicine communications. 2005;26(10):881-884.

86. de Amorim e Silva CJ, Mackenzie A, Hallowell LM, Stewart SE, Ditchfield MR. Practice MRI: reducing the need for sedation and general anaesthesia in children undergoing MRI. Australasian radiology. 2006;50(4):319-323.

87. Mahajan L, Wyllie R, Steffen R, Kay M, Kitaoka G, Dettorre J, et al. The effects of a psychological preparation program on anxiety in children and adolescents undergoing gastrointestinal endoscopy. Journal of paediatric gastroenterology and nutrition. 1998;27(2):161-165.

88. Pressdee D, May L, Eastman E, Grier D. The use of play therapy in the preparation of children undergoing MR imaging. Clinical radiology. 1997;52(12):945-947.

89. Rosenberg DR, Sweeney JA, Gillen JS, Kim J, Varanelli MJ, O’Hearn KM, et al. Magnetic resonance imaging of children without sedation: preparation with simulation. Journal of the American Academy of Child and Adolescent Psychiatry. 1997;36(6):853-859.

90. Cepeda MS, Carr DB, Lau J, Alvarez H. Music for pain relief. Cochrane database of systematic reviews (Online). 2006(2):CD004843.

91. Richardson J, Smith JE, McCall G, Pilkington K. Hypnosis for procedure-related pain and distress in paediatric cancer patients: a systematic review of effectiveness and methodology related to hypnosis interventions. Journal of pain and symptom management. 2006;31(1):70-84.

92. Uman LS, Chambers CT, McGrath PJ, Kisely S. Psychological interventions for needle-related procedural pain and distress in children and adolescents. Cochrane database of systematic reviews (Online). 2006(4):CD005179.

93. Kleiber C, Harper DC. Effects of distraction on children's pain and distress during medical procedures: a meta-analysis. Nursing research. 1999;48(1):44-49.

94. Butler LD, Symons BK, Henderson SL, Shortliffe LD, Spiegel D. Hypnosis reduces distress and duration of an invasive medical procedure for children. Paediatrics. 2005;115(1):e77-85.

95. Liossi C, Hatira P. Clinical hypnosis versus cognitive behavioral training for pain management with paediatric cancer patients undergoing bone marrow aspirations. The International journal of clinical and experimental hypnosis. 1999;47(2):104-116.

96. Liossi C, Hatira P. Clinical hypnosis in the alleviation of procedure-related pain in paediatric oncology patients. The International journal of clinical and experimental hypnosis. 2003;51(1):4-28.

97. Liossi C, White P, Hatira P. Randomized clinical trial of local anaesthetic versus a combination of local anaesthetic with self-hypnosis in the management of paediatric procedure-related pain. Health Psychol. 2006;25(3):307-315.

98. Liossi C, White P, Hatira P. A randomized clinical trial of a brief hypnosis intervention to control venepuncture-related pain of paediatric cancer patients. Pain. 2009;142(3):255-263.

99. Harned RK, 2nd, Strain JD. MRI-compatible audio/visual system: impact on paediatric sedation. Paediatric radiology. 2001;31(4):247-250.

100.Train H, Colville G, Allan R, Thurlbeck S. Paediatric 99mTc-DMSA imaging: Reducing distress and rate of sedation using a psychological approach. Clinical radiology. 2006;61(10):868-874.

101.Green SM, Krauss B. Barriers to propofol use in emergency medicine. Annals of emergency medicine. 2008;52(4):392-398.

102.Green SM, Krauss B. Propofol in emergency medicine: pushing the sedation frontier. Annals of emergency medicine. 2003;42(6):792-797.

103. Miner JR, Burton JH. Clinical practice advisory: Emergency department procedural sedation with propofol. Annals of emergency medicine. 2007;50(2):182-187, $187 \mathrm{e} 181$.

104.Litman RS. Conscious sedation with remifentanil and midazolam during brief painful procedures in children. Archives of paediatrics \& adolescent medicine. 1999;153(10):1085-1088. 



\section{CHAPTER 4}

\section{Forced immobilization ('Restraint') during medical procedures in young children} An ethical and legal investigation of a common practice

Piet LJM Leroy* and Rankie (M) M ten Hoopen*

\footnotetext{
* Both authors made an equal contribution to this article.
} 


\section{Abstract}

It is frequently the case that children need to be physically restrained in order to allow medical procedures to be carried out. It is safe to assume that restraint is part of daily practice in the provision of medical care to children. However, the use of restraint in sick children raises important objections.

This paper lists the objections and summarizes the relevant scientific literature and available guidelines on this topic. The use of restraint is tested against ethical and legal considerations in the field of health care. The decision on whether to apply restraint must be approached from the perspective of the quality of care. All patients, including children, are entitled to good medical care. In non-lifesaving conditions, healthcare professionals facing the need for procedural restraint of an individual child must firstly consider all possible alternatives and next, choose the best option. The use of restraint is only acceptable if it is unquestionably the best alternative. 


\section{Introduction}

For medical procedures that require the patient to sit or lie still, young children often need help. By using a certain level of force, health professionals can achieve or support the necessary level of immobility. In literature, this action is called 'therapeutic holding'. ${ }^{1}$ It is nevertheless not uncommon for children to resist this, because they experience the procedure as frightening and/or painful. If a necessary procedure threatens to go wrong as a result, the decision may be taken to restrain the child, thereby physically forcing it to undergo the procedure. This method is known in literature as 'restrictive physical intervention' or simply 'restraint'. ${ }^{1}$

The application of restraint probably forms part of day-to-day paediatric care (table 1). The practice of applying restraint nevertheless faces a number of objections. By applying restraint, the professional consciously acts counter to the child's wishes. Since the procedure (for which restraint is applied) is after all in the child's interest, a difficult dilemma ensues: does the child's medical interest warrant ignoring or overpowering the child's resistance? In acute lifethreatening situations, it seems only natural to protect a non-cooperative child against itself and to force it to undergo necessary procedures. But what about procedures that are less urgent? Is it acceptable under such circumstances to restrain a child if better alternatives are available (table 2) and moreover if it is unlikely that a temporary delay of the treatment (in anticipation of applying the alternative) would adversely affect the child?

Table 1: Possible grounds for restraint during medical procedures in children

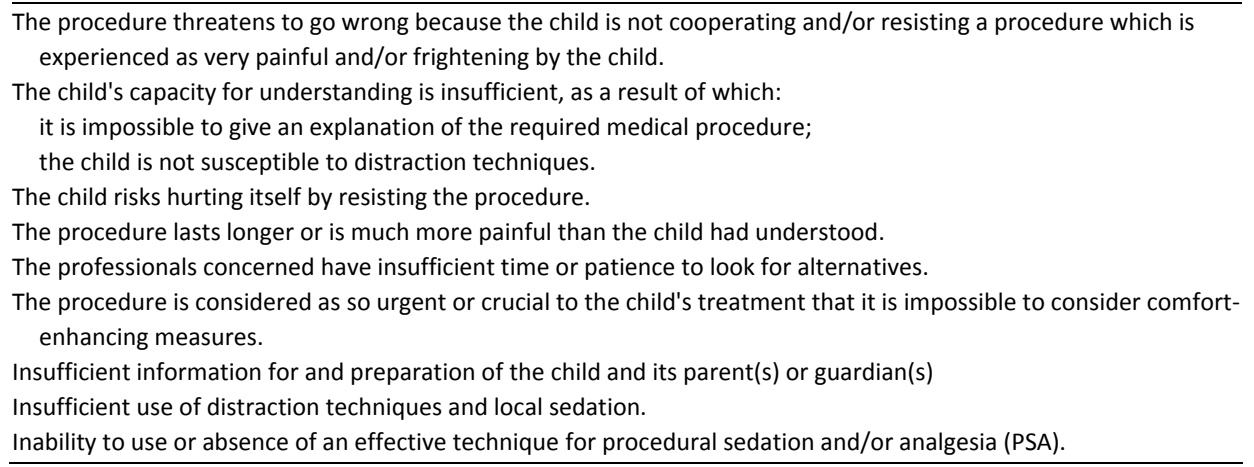

A young child that cannot yet understand the significance and objective of a specific treatment is unable to refuse or accept such treatment. It can nevertheless be aware of (the threat of) an impending treatment and understand the situation to that extent. Does the child have any right to stand up for its own will and - more importantly - how should a health worker deal with that right, if it exists?

Lastly, it is important to note that restraint is not a standard part of a medical treatment, but in fact a separate, additional treatment. Should a professional applying restraint therefore have a demonstrable competency for it, and should restraint be specified in protocols, partly for the same reason? Should the application of restraint be recorded in the patient's notes and, also important, should it be preceded by a fully informed consent, including an offer of alternative options? 
Table 2: Measures and provisions to optimize procedural comfort

\begin{tabular}{|c|c|}
\hline Strategy & Examples \\
\hline Preventive measures & $\begin{array}{l}\text { Avoid superfluous procedures. } \\
\text { Only allow an experienced professional to carry out procedures. } \\
\text { Agree a maximum number of attempts at the procedure in advance. } \\
\text { Early insertion of a central venous line under general anaesthesia (for example, } \\
\quad \text { during long-term treatment with intravenous antibiotics). }\end{array}$ \\
\hline $\begin{array}{l}\text { Optimal local and topical anaes } \\
\text { thesia }\end{array}$ & $\begin{array}{l}\text { Allow sufficient time for topical anaesthesia to become effective (for example, at } \\
\text { least } 60 \text { minutes for EMLA }{ }^{\oplus} \text { ). } \\
\text { Apply topical anaesthesia to the correct location. } \\
\text { Implementation of new topical anaesthetic techniques. }{ }^{18} \\
\text { For infiltration with lidocaine: buffer lidocaine with bicarbonate and use the small- } \\
\text { est possible needle to significantly reduce the pain upon infiltration. }{ }^{19}\end{array}$ \\
\hline $\begin{array}{l}\text { Non-pharmacological tech- } \\
\text { niques }\end{array}$ & $\begin{array}{l}\text { Optimal positioning of the child. }{ }^{20} \\
\text { Presence of the parent(s) or guardian(s). } \\
\text { Preparation, game therapy. } \\
\text { Distraction techniques and hypnosis. }\end{array}$ \\
\hline $\begin{array}{l}\text { Ready availability of effective } \\
\text { Procedural Sedation and/or } \\
\text { Analgesia (PSA) }\end{array}$ & $\begin{array}{l}\text { Light sedation for 'small' procedures (e.g. blends of nitrous oxide and oxygen). } \\
\text { Deep, titratable sedation for very painful procedures (e.g. propofol). } \\
\text { Professionals trained in PSA. }\end{array}$ \\
\hline Rescue Anaesthesia & $\begin{array}{l}\text { Availability of anaesthesia if other techniques appear or turn out to be ineffective } \\
\text { or unsafe. }\end{array}$ \\
\hline
\end{tabular}

\section{Restraint in medical literature}

In 2007, Brenner summarized the literature on this subject. She pointed out a lack of research and concluded that this demonstrates that medical professionals tend to ignore the existence and relevance of restraint or consider it a taboo subject. ${ }^{2}$ Various authors argue for more research, to shed light on medical grounds, age-related techniques, alternatives, required training for professionals, and the ethical and legal framework. ${ }^{3-8}$

In a few publications, restraint (in terms of the effects it has) has been associated with speech and language problems, a negative self-image, fear of and distrust of medical care, and with post-traumatic stress disorder. ${ }^{2}$ According to paediatric nurses, restraint is more traumatic for a child than the treatment itself. ${ }^{9}$ Longitudinal research with leukemia patients has shown that any participation by parents in restraint has a negative effect on the relationship with their child. $^{10}$

\section{Existing guidelines on restraint}

The guideline 'Safe sedation of children undergoing diagnostic and therapeutic procedures' from the Scottish Intercollegiate Guidelines Network calls restraint during a procedure that is not lifesaving unacceptable. ${ }^{11} \mathrm{~A}$ recent British guideline for paediatric nurses states that restraint must only be used to prevent serious injury to the child or to bystanders. According to this guideline, 
restraint must meet a number of basic principles, including the prevention of unnecessary procedures, setting a low threshold for using PSA, determining in advance the maximum number of attempts to perform the procedure, access to a training course and protocols, full informed consent from parents/carers, rigorous documentation of the procedure, and a subsequent evaluation of how the child, parents, and staff experienced it. ${ }^{1}$ As far as we could ascertain, the British Society of Paediatric Dentistry is the only one of all the medical occupational groups to have drawn up and published a policy document on the use of restraint. Based on ethical and legal considerations, the document recommends extreme reticence in applying restraint. ${ }^{12}$

\section{Ethical considerations}

Three important ethical basic principles in health care are: first and foremost, non-maleficence ('primum non nocere') and beneficence, respect for life, and respect for autonomy. When a painful procedure needs to be performed on a child that resists, it is crucial to weigh off whether applying restraint is in the child's interest. Creating psychological trauma is not in the child's interest; it could actually harm the child. Moreover, knowledge and technology are available for most procedures to make them (more) comfortable for the child. In this perspective, it is unethical to deny the child these techniques and using restraint on a fearful child cannot actually be fair. ${ }^{13}$ If the methods concerned are not immediately available, the question arises as to whether postponing the treatment would harm the patient's condition. In most non life-threatening situations, this will not be the case and sufficient time is available to look for an alternative without using restraint.

\section{Legal considerations}

In the Netherlands, there are no specific legal regulations concerning resistance put up by young children. The section in the Dutch Civil Code (BW) relating to the Medical Treatment Contracts Act (WGBO) contains only one provision on resistance put up by patients aged twelve and older incapable of giving legal consent (article 7:465 clause 6 BW). However, a special regime is nevertheless in place for the provision of information and obtaining of consent. For children up to the age of twelve, only the parent(s) or guardian(s) are required to give their consent for medical procedures to take place (article 7:450 and 7:465 clause $1 \mathrm{BW}$ ). In addition to the parent(s) or guardian(s), the child itself is nevertheless entitled to information, to be provided in a manner appropriate for its ability to understand (article 7:448 clause $1 \mathrm{BW}$ ). This means that a procedure for which consent has been given is allowed to take place even if the child resists. But is this really the case?

The International Convention on the Rights of the Child (ICRC), which became effective in the Netherlands on 8 March 1995, starts out from a number of fundamental principles regarding the interests and (legal) position of children in relation to available facilities, protection, and participation (including: freedom of opinion), also in relation to health care (articles 3 and 24) (table 3). Although not all provisions of the ICRC have a direct effect on health care, they serve to educate our State and our citizens (including health care workers) regarding an important body of ideas: for any measures relating to children, the interest of the child itself is paramount. As far as health care is concerned, this means that children are entitled to the best possible care. 
In the Netherlands, health care workers are bound by the general standard of 'good care provision' (article 7:453 BW). Applying restraint is therefore only permitted if it is absolutely certain to constitute the most adequate form of care in the absence of a better or practical alternative. Since restraint is no longer a standard component of a medical procedure, it also requires separate informed consent from the parent(s) or guardian(s). In respect of the child itself, good care provision means that in the event of resistance, the care provider must first use dialogue to try and convince the child of the importance of the treatment in order to gain as much trust and cooperation as still possible. Useful guidelines, hints, and tips for dealing and communicating with children can also be found in the model guideline for care providers in relation to information and consent for under-age patients ${ }^{14}$ and in the Code of Behavior in the event of resistance from under-age patients taking part in medical research, which is also very useful with regard to general clinical situations. ${ }^{15}$

Table 3: Articles from the Convention on the Rights of the Child*, that may apply to restraint during medical procedures.

Article 3, clause 1: "In all actions concerning children, whether undertaken by public or private social welfare institutions, courts of law, administrative authorities, or legislative bodies, the best interests of the child shall be a primary consideration".

Article 12, clause 1: "The child who is capable of forming his or her own views shall have the right to express those views freely in all matters affecting the child, the views of the child being given due weight in accordance with the age and maturity of the child".

Article 19, clause 1: "Children will have the right to be protected against all forms of physical or mental violence, both within the family and outside it".

Article 24, clause 3: "All effective and appropriate measures will be taken with a view to abolishing traditional practices prejudicial to the health of children".

* The ICRC was unanimously accepted on 20 November 1989 by the General Meeting of the United Nations and became effective in September 1990. For the Netherlands, the Convention took effect on March 8, 1995

Recent jurisprudence shows that a care provider who does not allow sufficient time and effort to adopt a suitable approach to a resisting child may have to face the - negative - consequences: courts may rule that a defensive (panic) response from children resulting in injuries to the care provider is not unlawful. In that case, any claim for damages against the parent(s) would fail. ${ }^{16}$ Seen from the child's perspective, it could furthermore be argued that the child has a right to oppose a medical treatment, at least within certain specific boundaries (to what extent could the defensive behavior have been expected? How serious was the injury caused?).

The norm of good care provision returns to the denominator of the right of the patient or client to 'good care' which is set to largely replace section 7.7.5 of the Dutch Civil Code (WGBO): the Clients' Rights (Care Sector) Act (WCZ). ${ }^{17}$ Not explicit, but implied in the draft legislation for the WCZ, is nevertheless the provision set out in article 7:448 clause 1 of the Civil Code, namely that a child under twelve years of age must be informed in accordance with his or her level of comprehension. According to the legislator, the right to be provided with information about the treatment, associated with the patient's or client's right to express his or her own opinion on the treatment, already follows from the right to receive good care. This view is susceptible to criticism. Particularly when young children are concerned, whose fear and tendency to protest call for a particularly careful approach, it must not be a mere formality to imprint on care providers' 
minds that they must proceed with the necessary care and prudence. This requires more than a broadly formulated, open standard ('good care'). Adopting a confidence-building approach and providing information come top of the list.

\section{New draft guidelines on Procedural Sedation and Analgesia (PSA) outside the operating theatre}

Under instructions from the Dutch Healthcare Inspectorate, the Netherlands Society of Anaesthesiologists (NVA) and the Dutch Society of Paediatrics (NVK), a working group with representatives of 21 professional associations with support from the Dutch Institute for Healthcare Improvement ( $\mathrm{CBO}$ ), have drawn up evidence-based guidelines for PSA. These guidelines are a follow-up to guidelines issued in 1998 and set out the conditions for safe and effective PSA in adults, children, and intensive-care patients. In the meantime, all professional associations have expressed their agreement with the content of the final draft version, which is now only waiting for ratification by the NVA and NVK. The guidelines state that applying restraint to children during non life-saving procedures is in principle unacceptable, unless it is certain that it amounts to providing the best possible care.

\section{Conclusions and recommendations}

Everyone is entitled to good care, including children. The application of physical restraint must be approached from the viewpoint of quality of care. Unless the child's life is at stake, health care providers encountering resistance from children against a procedure must first consider all possible alternatives and then opt for the most appropriate care for the case. If restraint is applied nonetheless, it must be carried out within a strict, transparent framework. This includes determining in advance the duration of the treatment and the number of attempts. Health care providers must be given good-quality training, including in adequate protocols. Furthermore, the parent(s) or guardian(s) must be asked for their full, informed consent, the treatment must be rigorously documented and motivated in the patient notes and the experiences of the child, parents, and attending care staff must be evaluated.

The above can only be achieved if functional alternatives are widely available and safe, including effective PSA techniques (table 2). It is therefore hoped that anaesthesiologists and paediatricians (NVA and NVK) will assume responsibility by making it a priority to ensure that the new guidelines can be applied in practice. 


\section{References}

1. Royal College of Nursing. 'Restrictive physical intervention and therapeutic holding for children and young people'. Guidance for nursing staff. http://www.rcn.org.uk/_data/assets/pdf_file/0016/312613/003573.pdf last accessed 2010.

2. Brenner M. 'Child restraint in the acute setting of pediatric nursing: an extraordinarily stressful event'. Issues in comprehensive pediatric nursing. 2007;30(1-2): 29-37.

3. Hardy M, Armitage G. 'The child's right to consent to X-ray and imaging investigations: issues of restraint and immobilization from a multidisciplinary perspective'. J Child Health Care. 2002;6(2): 107-119.

4. Jeffery K. 'Therapeutic restraint of children: it must always be justified'. Paediatric nursing. 2002;14(9): 20-22.

5. Pearch J. 'Restraining children for clinical procedures'. Paediatric nursing. 2005;17(9): 36-38.

6. Tomlinson D. 'Physical restraint during procedures: issues and implications for practice'. J Pediatr Oncol Nurs. 2004;21(5): 258-263.

7. Folkes K. 'Is restraint a form of abuse?' Paediatric nursing. 2005;17(6): 41-44.

8. Collins P. 'Restraining children for painful procedures'. Paediatric nursing. 1999;11(3): 14-16.

9. Robinson S, Collier J. 'Holding children still for procedures'. Paediatric nursing. 1997;9(4): 12-14.

10. McGrath P, Forrester K, Fox-Young S, Huff N. "Holding the child down” for treatment in paediatric haematology: the ethical, legal and practice implications'. Journal of law and medicine. 2002;10(1): 8496.

11. SIGN Guideline 58: Safe sedation of children undergoing diagnostic and therapeutic procedures http://www.sign.ac.uk/pdf/sign58.pdf. Published 2004.

12. Nunn J, Foster M, Master S, Greening S. 'British Society of Paediatric Dentistry: a policy document on consent and the use of physical intervention in the dental care of children'. International journal of paediatric dentistry / the British Paedodontic Society [and] the International Association of Dentistry for Children. 2008;18 Suppl 1:39-46.

13. Cravero JP. 'Pride, Prejudice, and Pediatric Sedation: A Multispecialty Evaluation of the State of the Art'. Report from a Dartmouth Summit on Pediatric Sedation. September 9th, 2000. http://www.npsf.org

14. Appendix 7 to: Witmer, H. and Roode, M. de, 'Van wet naar praktijk. Implementatie van de WGBO. Deel 2. Informatie en Toestemming' (From act to practice: Implementation of the Medical Treatment Contracts Act. Part 2. Information and Consent), Utrecht: KNMG 2004; 69-74 and 75-90 (notes and points of interest).

15. Central Committee on Research Involving Human Subjects (http://www.ccmo-online.nl/hipe/uploads/downloads/verzet_minderjarigen.doc).

16. Court of 's-Hertogenbosch, 26 January 2011, Medisch Contact 2011; 808-810.

17. Legislative bill Clients' Rights (Care Sector) Act, Parliamentary Papers II 2009/10, 32 402, no. 2.

18. Zempsky WT. 'Pharmacologic approaches for reducing venous access pain in children'. Pediatrics. 2008;122 Suppl 3:S140-153.

19. Davies RJ. 'Buffering the pain of local anaesthetics: A systematic review'. Emergency medicine (Fremantle, WA. 2003;15(1): 81-88.

20. Sparks LA, Setlik J, Luhman J. 'Parental holding and positioning to decrease IV distress in young children: a randomized controlled trial'. Journal of pediatric nursing. 2007;22(6): 440-447.

21. Een kind in het nauw maakt rare sprongen (Desperate needs lead to desperate deeds). Medisch Contact. 2011;66(13): 808-810. 


\section{Appendix bij hoofdstuk 4 \\ Een kind in het nauw maakt rare sprongen}

Piet Leroy, Rankie ten Hoopen, Hans Knape. Medisch Contact 2011; 66(20): 1284

De rechtbank in 's Hertogenbosch wees recentelijk een vordering af van een tandarts die gewond raakte bij een poging een vierjarig kind te behandelen. ${ }^{21}$ Het letsel ontstond door een afweerreactie van het kind. De vader was niet aansprakelijk omdat de paniekreactie niet onrechtmatig was. De uitspraak is bijzonder omdat zij de facto aan het kind een recht op verzet toekent. Het schaderisico ligt bij de hulpverlener als deze op de reactie bedacht kan zijn en veiligheidsmaatregelen kan treffen.

Maar hoe moet een arts met verzet van een kind omgaan? Voor een volwassene is het niet moeilijk om het verzet met kracht te doorbreken. Deze praktijk, 'restraint' genaamd, behoort tot de dagelijkse kindergeneeskundige zorg. Beschikbare comfortbevorderende methoden worden vaak niet toegepast. Ethisch is dit moeilijk te verdedigen. Voor verzet door kinderen (wilsonbekwame minderjarigen van 12 jaar en ouder daargelaten) bestaat geen wettelijke regeling. Daardoor kan een verrichting waarvoor de ouders toestemming hebben gegeven ondanks verzet gewoon doorgaan. Echter, een hulpverlener is gebonden aan de norm van goed hulpverlenerschap (artikel 7:453 BW) en dient het recht van het kind op de best mogelijke zorg te waarborgen. Restraint toepassen kan dan alleen als vaststaat dat er geen alternatief is en na instemming van de ouders.

De nieuwe conceptrichtlijn Procedurele Sedatie en/of Analgesie buiten de operatiekamer stelt dat restraint bij niet-levensreddende handelingen ontoelaatbaar is. Dit is alleen te realiseren als effectieve technieken voor lichte en diepe sedatie veilig inzetbaar zijn. Het is daarom te hopen dat anesthesiologen en kinderartsen hun verantwoordelijkheid nemen door er met voorrang voor te zorgen dat de richtlijn ook in de praktijk toepasbaar wordt. 


\section{Een kind in het nauw maakt rare sprongen}

In het leven van een kind zijn de bezoekjes aan de dokter doorgaans niet de meest vreugdevolle episodes. Een kind is niet geneigd tot het maken van een vreugdesprong bij een injectie in de arm, een neusendoscopie of druppels in de ogen of oren. Zeker na een - in de ogen van een kind - traumatische ingreep is de spontane bereidheid tot medewerking aan een vervolgbehandeling meestal niet erg groot. Zo ook bij de 4-jarige jongen in onderstaande casus. De week daarvoor was bij hem, nota bene zonder verdoving, een kies getrokken. En alweer mocht hij zitting nemen op de stoel van de tandarts, ditmaal voor het vullen van een kies.

Dat de jongen de kaken stijf op elkaar hield en de handen voor de mond, zal een gemiddelde arts niet verbazen. Ook nadat de tandarts tien minuten op de jongen had ingepraat en de hulp van een tweede assistente had ingeroepen, bleef de jongen zich verzetten. De tandarts vond het daarop welletjes geweest. Nog voor de komst van de tweede assistente hield hij beide handen van de jongen vast om zo alsnog de ingreep te kunnen verrichten. De tandarts had er evenwel geen rekening mee gehouden dat de angstige jongen in staat was een hand los te rukken. Daarbij verdraaide de tandarts zijn duim.

De tandarts stelde daarop de vader van de jongen aansprakelijk voor het letsel. De rechter gaat hierin niet mee. De paniekreactie van de jongen was niet geheel onverwacht. Het is bovendien onduidelijk waarom de tandarts niet heeft gewacht totdat de tweede assistente was gearriveerd. De rechter vindt het voorts veelbetekenend dat de jongen zich na de komst van deze assistente zonder problemen heeft laten behandelen.

Les uit onderstaande zaak is dat $u$ ook rekening moet houden met de gevoelens van kleine kinderen. Het geven van informatie over de behandeling, waartoe u ook bij kinderen verplicht bent, waarborgt nog geen medewerking. Daarvoor zijn andere vaardigheden vereist.

\section{B.V.M. Crul, arts}

prof. mr. A.C. Hendriks, jurist KNMG 


\section{CHAPTER 5}

\section{Summary of the new evidence based guideline on Procedural Sedation and/or Analgesia (PSA) in children at locations outside the operating theatre}

The final version of this guideline has been approved by all participating scientific societies and is currently pending authorization by the Netherlands Society of Anaesthesiologists Dutch Society of Paediatrics.

Piet Leroy, Daphne Schippers, Hans Knape

Initiators

Netherlands Society of Anaesthesiologists

Dutch Society of Paediatrics

Organization

Dutch Institute for Healthcare Improvement (Central Accompaniment Organization (CBO)

October 2010 


\section{Introduction}

At the request of the Public Health Inspection Service, the Netherlands Society of Anaesthesiologists, and the Dutch Society of Paediatrics, a working group comprising representatives of 21 professional associations compiled new guidelines for procedural sedation and/or analgesia (PSA), with support from the Dutch Institute for Healthcare Improvement CAO. These guidelines continue where the guidelines issued in 1998 left off. The current guidelines stand out because they include a section on adult patients, a section on patients in intensive care, and a section concerning children.

\section{Methodology}

The guidelines were drawn up using the method of evidence-based guideline development (EBGD), which means that conclusions were drawn from existing scientific evidence, in addition to other practical considerations, in order to formulate recommendations for daily practice. This process took place between June 2006 and January 2008. The literature search included publications until September 2007. A first draft was discussed with the boards of the two initiating scientific societies. Next, the guideline text was peer-reviewed by members of all participating scientific societies. A final version was presented to all participating societies by the end of 2009. By October 2010, this guideline's content was approved by all participating societies. Currently the guideline is waiting for final authorization and implementation by the two initiators.

\section{Guideline working group}

- P.L.J.M. Leroy, paediatrician (chairman), Nederlandse Vereniging voor Kindergeneeskunde (Dutch Society of Paediatrics)

- M.E.A. van Bergen-Rodts, Landelijke Vereniging Kind en Ziekenhuis (National association for children in hospitals)

- H.E. Blokland-Loggers, paediatrician, Nederlandse Vereniging voor Kindergeneeskunde (Dutch Society of Paediatrics)

- J.J. Dogger, anaesthetic nursing staff, Nederlandse Vereniging van Anaesthesiemedewerkers (Dutch association of anaesthetic nursing staff)

- M.P. Gorzeman, Emergency physician, Nederlandse Vereniging van Spoedeisende Hulp Artsen (Dutch association of accident and emergency doctors)

- M.M.L. van der Hall, remedial educationalist, Beroepsvereniging voor Orthopedagogen en Klinisch pedagogen met een Academische Opleiding (Professional association of special needs educationalists and academically-trained clinical educationalists)

- F.T.M. ten Have, anaesthesiologist, Nederlandse Vereniging voor Anaesthesiologie (Netherlands Society of Anaesthesiologists)

- G. Jorna, paediatric nurse and nurse practitioner paediatric neurology, Vereniging Verpleegkundigen en Verzorgenden Nederland Afdeling Kinder Verpleegkundigen (Association for nurses and carers in the Netherlands, section for paediatric nurses)

- A.Y.N. Schouten-van Meeteren, paediatric hemato-oncologist, Nederlandse Vereniging voor Kindergeneeskunde (Dutch Society of Paediatrics)

- M.H. Rövekamp, paediatrician, Nederlandse Vereniging voor Kindergeneeskunde (Dutch Society of Paediatrics) 
- I.N. Snoeck, paediatric neurologist, Nederlandse Vereniging voor Kinderneurologie (Dutch association for child neurology)

- L.K.P. Tielens, paediatric anaesthesiologist, Nederlandse Vereniging voor Anaesthesiologie (Netherlands Society of Anaesthesiologists)

- J.I.M.L. Verbeke, paediatric radiologist, Nederlandse Vereniging voor Radiologie (Dutch association for radiology)

- J.S.J. Veerkamp, dentist, Nederlandse Maatschappij ter Bevordering der Tandheelkunde (Dutch society for the promotion of dentistry)

- D.M. Schipper, consultant-methodologist CBO (secretary)

- T.A. van Barneveld, program director, CBO (until september 2008)

\section{Content}

These guidelines describe the conditions for optimally safe and optimally effective PSA in children. Both objectives (safety and effectiveness) form the recurring theme of the guidelines.

The guidelines consist of recommendations for PSA in children (age limit from 44 weeks postconceptional age to 15 years) at locations outside the operating theatre, setting out the underlying evidence for the recommendations.

The following subjects fall outside the scope of the guideline:

- PSA in (premature) neonates ( $<4$ weeks post-term age)

- All forms of PSA in intensive care for children

- All forms of PSA for the intubation of children

- All forms of PSA for palliative and/or termination situations

- All forms of pre-anaesthesia PSA

- All forms of postoperative analgesia

- All forms of psychiatric PSA

Although this directive primarily relates to procedures and actions occurring inside hospitals, the guidelines also apply to sedations outside hospitals, for example in dentistry and independent treatment centers (ZBC).

This document is a brief summary of the Guidelines for Sedation and/or Analgesia in Children at Locations outside the Operating Theatre. For a comprehensive description of the literature search, summary of the relevant literature, the levels of evidence and all references used, we refer to the full text of the guidelines.

\section{Part 1 Recommendations on the preconditions}

\subsection{Definitions}

\section{What is PSA?}

Procedural Sedation and/or Analgesia (PSA) refers to: "the prescription or administration outside the operating theatre (usually by a non-anaesthesiologist) of a sedative and/or sedating analgesic in the context of a diagnostic or therapeutic procedure for the purpose of keeping a patient sufficiently calm, and free of fear and pain, in order to allow the surgery to go ahead comfortably and efficiently." (Krauss and Green, Lancet 2006) 
PSA must be viewed as an independent medical treatment

Those performing PSA will need to be demonstrably competent in that area.

\section{Sedation and analgesia are separate concepts}

It is fundamentally wrong to combat pain exclusively with an anxiolytic agent or sedative. A topical and/or systemic analgesic must always be used in the event of painful procedures. Using only sedation for painful procedures is not acceptable, even if it is in the hopeful anticipation that the substance will have an amnesic effect.

\section{Definition of safe as well as comfortable procedures in children}

These guidelines formulate recommendations for safe and effective PSA. These recommendations must be considered as only one component of a larger, comprehensive policy, in order to ensure that medical procedures on children are optimally safe and comfortable. Table 1 contains the six cornerstones of this comprehensive policy.

Table 1: Cornerstones of a comprehensive policy towards procedural comfort in children

1. Prevention of procedural pain and stress.

2. An active policy in relation to the prevention of forced securing and restraint.

3. Optimal use of effective forms of local or topical anaesthesia.

4. The systematic application of effective non-pharmacological techniques (preparation, distraction, hypnosis, etc.).

5. The application of the most adequate PSA technique, individually titrated and carried out by a trained professional.

6. A local policy towards the ready availability of the so-called "rescue anaesthesia" if a PSA technique turns out to be inadequate or if it can be anticipated that the available PSA techniques may be insufficient or unsafe in an individual patient.

\section{Levels of sedation depth}

In recent medical literature, a distinction is made between various levels of sedation. (Table 2) The following observations need to be made with the definitions below:

1. These definitions apply to children with an ASA* I and II classification, over one year old. Other criteria may apply to other categories of patients (patients with a mental disability, patients with comorbidity leading to ASA III and ASA IV, patients with an abnormal airway, and patients $<1$ year).

2. The definitions must not give the impression that the levels of sedation can be incrementally discerned, or are individually predictable. The anticipated level of sedation is reasonably predictable only in the event of a light sedation/anxiolysis with ketamine. In all other cases, the sedation level is rather a continuum and the patient may reach a deeper sedation level than was intended.

\footnotetext{
* Physical Status Classification compiled by the American Society of Anesthesiologists (ASA) and internationally used for risk stratification in relation to anaesthesia. Most international guidelines recommend that this classification is also used for screening PSA (see Table 3 below).
} 
Table 2: definitions of different levels of sedation

1. Light sedation/anxiolysis: Two states that are difficult to tell apart, in which the anxiety and stress level of the patient have been lowered while the patient remains basically fully conscious. The patient responds adequately and consistently to verbal stimuli, and verbal communication therefore remains possible. This state is associated with few risks in patients without significant comorbidity. Although cognitive functions and coordination are reduced, ventilatory and cardiovascular functions remain unaffected. Light sedation/anxiolysis is typically a state of mind that occurs after 1 standard dose of midazolam $(0.1 \mathrm{mg} / \mathrm{kg}$ intravenously or $0.2-0.5 \mathrm{mg} / \mathrm{kg}$ transmucosally) and with nitrous oxide sedation (inhalation concentration up to 50\%). Higher doses, other medicines, and combinations with other analgesics will virtually always lead to a deeper sedation level.

2. Moderate sedation: Pharmaceutically induced reduction in awareness, during which the patient still responds purposefully when spoken to, or to light tactile stimuli. In this stage, no interventions are needed to keep the airway open, airway reflexes are intact, and ventilation is adequate. If the response is not clearly adequate and purposeful but more of a withdrawal reflex, we speak of deep sedation.

3. Deep sedation: This is a pharmaceutically induced decline in awareness, during which the patient does not respond to being spoken to, but reacts purposefully to repeated or painful stimuli. Airway reflexes and ventilation may be reduced and it may be necessary to keep the airway open. The concept of "deep sedation" is a contested term because the distinction with anaesthesia becomes less clear. A typical example is the deep sedation caused by propofol, during which it is possible, with the necessary expertise, to keep spontaneous respiration going and the airway open. The risk of reduced breathing is more or less a linear function of the dose and depth of sedation.

4. Dissociative sedation: Also called a trance-like cataleptic sedation, it is typically the result of sedation with ketamine. As far as the depth of sedation, analgesia, and response level is concerned, ketamine causes a state that primarily corresponds to anaesthesia. However, contrary to anaesthesia, the airway reflexes, respiration and hemodynamics largely remain intact, even at comparatively high doses. It makes ketamine attractive for use in PSA, particularly for painful procedures.

5. General anaesthesia: A pharmaceutically induced state of unconsciousness, in which the patient is unresponsive, even to painful stimuli. The ability to keep the airway open will often be reduced or absent, and ventilation will frequently be depressed, consequently requiring support. Cardiovascular functions may also be impaired. Can only be applied under the personal supervision of an anaesthesiologist.

3. In these guidelines, a clear link is made between the level of sedation and the necessary professional competences and other preconditions. Anxiolysis and light sedation require other competences and preconditions than do moderate and deep sedation. As far as PSA in children is concerned, it appears from existing literature that moderate and deep sedation are not easily discernible, in terms of predictability and controllability. Furthermore, a large possibility exists with children that a deliberately moderate level of sedation is insufficient and that PSA consequently needs to be "scaled up" to deep sedation. For that reason, these guidelines impose the same levels of expertise, competence, and preconditions for performing both moderate and deep sedation in children.

A formal training regime with accreditation and special instrumental and logistic facilities are essential for performing PSA safely in selected patients. Hence, the following recommendations:

\section{Recommendation 1}

PSA must only be performed by a professional who has the demonstrable competence. 


\section{Recommendation 2}

In all cases, it is necessary to opt for the most effective PSA technique:

1. The nature of the procedure to be performed, the individual sedation requirement of the patient, and the patient's state of health determine which sedation level and which PSA technique (sedation and/or analgesia) is most appropriate to make the intervention as successful as possible in a manner that is as comfortable and safe as possible for the individual patient. Optimally successful means that the PSA technique aims for a $100 \%$ predictable procedural success and timing, high predictability of the sedation level and duration, and minimal induction and recovery times. Optimally comfortable means minimal to absent pain and anxiety for the patient, and the absence of a need for restraint.

2. For (very) painful and/or (very) stressful procedures and for procedures in young children that require prolonged immobility or cooperation, deep sedation may be required.

3. Effective systemic and/or topical analgesics must be used on every occasion for painful procedures. Using only sedation for painful procedures is not acceptable, even if it is the expectation that the sedative will have an anterograde amnesic effect.

4. The aforementioned aspects (i.e. intended sedation level, applied sedation technique, individual patient characteristics, and state of health) determine the necessary preconditions and the necessary competencies of the medical staff involved (see the specific recommendations in the chapters on competencies, monitoring, rescue facilities, and training).

\section{Recommendation 3}

For the optimal procedural comfort in children, it is not sufficient for an optimal PSA technique to be available and deployable. Prevention of painful/stressful procedures, banishing securing and restraint, optimal application of local/topical anaesthesia, non-pharmacological techniques, and rescue anaesthesia must also be incorporated in a comprehensive policy aimed at safe and comfortable care.

\section{Recommendation 4}

The working group recommends that the four sedation levels of PSA are divided into two separate pragmatic categories: one category for light sedation/anxiolysis and another category for moderate and deep sedation. Various levels of expertise, competency, and preconditions are required for both categories of sedation levels.

\subsection{Patient selection, fasting, and informed consent}

\subsubsection{Patient selection and screening}

Since PSA is a second medical procedure in addition to the diagnostics or treatment for which PSA is needed, the risks associated with both procedures must be weighed off. In this regard, it is necessary to identify the factors associated with the procedure and patient, or a combination of both, that may contribute to an increased risk arising from the totality of these medical procedures. 
Indications can be found in relevant literature to the effect that children with an ASA classification above II, children under one year old, and children with an underlying (respiratory) condition have an increased risk of complications in the context of PSA. In addition, there are indications that the absence of a systematic preprocedural screening of the basic condition and any existing risks constitute a significant risk factor for fatal or serious complications arising during PSA. Other recommendations in relation to patient selection and screening are particularly based on indirect evidence (derived from the standard precautions during anaesthesia) and common sense. The working group has drawn up two separate lists of (relative) risk factors based on its literature review. Table 4 contains risk factors associated with an increased risk of complications. Table 5 contains risk factors associated with an increased risk of PSA being ineffective.

\section{SCREENING}

\section{Recommendation 5}

On the occasion of every PSA, a systematic evaluation of the patient and a risk assessment must be carried out prior to the procedure. A written evaluation report must be added to the patient notes, containing at least:

An anamnesis of health problems, airway problems, previous experiences with PSA or anaesthetics, and allergies or intolerance to the medicines to be used.

A physical examination, concentrating on vital functions and the quality of the airway.

A formal risk assessment, which can be carried out using the "ASA physical status classification" (Table 3). 
Table 3: ASA Physical status classification for children

\begin{tabular}{|c|c|c|}
\hline Status & Definition & (non-exhaustive) List with examples \\
\hline 1 & Healthy child & $\begin{array}{l}\text { No specific anamnesis } \\
\text { No maintenance treatment }\end{array}$ \\
\hline II & $\begin{array}{l}\text { Child with a mild systemic disease } \\
\text { without functional limitations }\end{array}$ & $\begin{array}{l}\text { Mild asthma without respiratory distress or need for oxygen } \\
\text { Disease with fever without organ-specific problems } \\
\text { Anemia without circulatory complaints } \\
\text { Epilepsy adequately controlled through medication (no recent fit) } \\
\text { (Corrected) cor vitium with normal heart function, without pul- } \\
\text { monary hypertension or respiratory problems } \\
\text { Controlled diabetes mellitus } \\
\text { Pleural empyema without respiratory distress } \\
\text { Malignity without respiratory, circulatory or neurological prob- } \\
\text { lems }\end{array}$ \\
\hline III & $\begin{array}{l}\text { Child with a serious systemic disease } \\
\text { as well as functional limitations }\end{array}$ & $\begin{array}{l}\text { Moderate to serious asthma with moderate respiratory distress } \\
\text { or need for oxygen } \\
\text { Bronchopulmonal dysplasia (BPD) with a low need for oxygen } \\
\text { Pneumonia } \\
\text { Epilepsy that is moderately controlled } \\
\text { (Corrected) cor vitium with light to moderately disturbed cardiac } \\
\text { function, light to moderate pulmonary hyptertension, or light } \\
\text { to moderate respiratory problems } \\
\text { Anemia with circulatory problems } \\
\text { Moderate diabetic keto-acidosis } \\
\text { Pleural empyema with moderate respiratory distress } \\
\text { Malignity with respiratory, circulatory, or neurological problems } \\
\text { Moderate obesity } \\
\text { Obstructive sleep apnea syndrome }\end{array}$ \\
\hline IV & $\begin{array}{l}\text { Child with a serious systemic disease } \\
\text { that is a constant threat to life }\end{array}$ & $\begin{array}{l}\text { Serious, life-threatening asthma } \\
\text { Serious BPD } \\
\text { Sepsis } \\
\text { Serious diabetic keto-acidosis } \\
\text { Any disease with far-reaching respiratory, circulatory, neurologi- } \\
\quad \text { cal, renal, and/or endocrine failures } \\
\text { Morbid obesity }\end{array}$ \\
\hline V & $\begin{array}{l}\text { Dying child not expected to survive } \\
\text { without intervention. }\end{array}$ & $\begin{array}{l}\text { Shock (hypovolemic, distributive, obstructive, cardiogene, or } \\
\text { dissociative) } \\
\text { Non-traumatic coma } \\
\text { Neurotrauma }\end{array}$ \\
\hline
\end{tabular}




\section{Recommendation 6}

1. In principle, only ASA I and II patients are eligible for PSA by a non-anaesthesiologist, on the express condition that these patients also have a normal airway, normal airway reflexes, and that there is no other demonstrable contra-indication (Table 4).

2. ASA III and IV patients and patients with a demonstrable contra-indication (Table 4) should preferably not have PSA administered by a non-anaesthesiologist in relation to the increased risk of complications.

In those cases it is recommended that an anaesthesiologist is consulted and that the procedure is only ever performed under PSA (a) provided the latter can be administered by a (child) anaesthesiologist or by a professional specifically trained in and credentialed for PSA and (b) provided the specific indications and contra-indications are carefully considered.

If the required quality and safety requirements in relation to professional competencies and preconditions are met, PSA can be carried out responsibly by a trained nonanaesthesiologist, provided it is done after consultation with and under the responsibility of an anaesthesiologist.

\section{Recommendation 7}

It is preferable not to consider patients with one or more of the conditions listed in Table 5 eligible for PSA, due to the increased risk of a laborious or ineffective sedation.

In that case, it is recommended that an anaesthesiologist is consulted and that the procedure is only ever performed under PSA (a) provided the latter can be administered by a (child) anaesthesiologist or by a professional specifically trained in PSA, and (b) provided the specific indications and contra-indications are carefully considered.

If the required quality and safety requirements in relation to professional competencies and preconditions are met, PSA can be carried out responsibly by a trained non-anaesthesiologist, provided it is done after consultation with and under the responsibility of an anaesthesiologist.

\section{PROCEDURE-DEPENDENT SELECTION CRITERIA}

The working group compiled a non-exclusive list of diagnostic and therapeutic procedures during which PSA can be performed, according to the relevant literature. (Table 6)

\section{Recommendation 8}

1. The factors that need to be considered in order to determine whether a procedure can be considered for a form of PSA, are the level of invasiveness and painfulness, the duration and complexity of the procedure, the expected effectiveness of the chosen form of PSA, and the anticipated individual response of the child to the procedure.

2. A form of PSA must be considered for any procedure that is not life-saving, or for which, despite maximum preparation, the patient's comfort and/or cooperation cannot be optimally guaranteed by local anaesthesia and the deployment of non-pharmacological techniques. If it is expected that PSA will not suffice, it is advisable to arrange access to 
low-threshold, readily available anaesthesia or to postpone the procedure until it can take place in more comfortable circumstances.

\section{Recommendation 9}

For procedures involving manipulation of the upper airway (for example, esophogagogastrescopy, laryngoscopy, and bronchoscopy), it is preferable to perform PSA under the supervision of an anaesthesiologist in view of the additional risk of respiratory complications.

Table 4: patient-specific risk factors for PSA-related complications

- ASA classification > II

- Abnormal airway (including large tonsils and anatomic defects of the upper or lower airway)

- Chronic lung disease

- Significant cardiac defects

- Increased intracranial pressure

- Decreased level of consciousness

- Anamnesis of sleep apnea

- Bulbar reflex defects

- Neuromuscular diseases or neurological disorders potentially leading to hypoventilation

- Defects in the kidney/liver function

- Gastroesophageal reflux or increased risk of choking

- Serious obesity

- Emergencies (and hence no empty stomach)

- Children under one year old, including neonates (until the age of one month if born at full-term) and premature children (until 60 weeks postconceptional age).

- Children already using opiates or sedatives

- Children using medication that may attenuate sedatives

- Children using anti-epileptic drugs

- Children with known allergies to sedatives

- Children who have previously suffered an adverse reaction

- No informed consent obtained from the parents/child itself

- Children offering limited cooperation and/or with learning difficulties

- Children that are too stressed, despite proper preparation

Older child with serious behavioural disorder

Table 5: patient-specific risk factors for an ineffective PSA

- Children in whom a previous PSA was ineffective

- Children offering limited cooperation and/or with learning difficulties

- Children that are too stressed, despite proper preparation

- Older children with serious behavioral disorders

- When it can be expected that the chosen form of PSA will be ineffective for a specific child (not optimally immobile during the full examination, not optimally comfortable for the patient, procedure only possible if restraint is used). 
Table 6: (non-exclusive) list of procedures for which PSA can be used

Oncology: Bone Biopsy (BB), Lumbar Puncture (LP), Bone Marrow Puncture (BM)

Radiology: Computer Tomography (CT) scan, Magnetic Resonance Imaging (MRI), MCG, echo(cardio)graphy.

Gastroenterology: Colonscopy, (gastroscopy - however, see recommendation 2)

Neurophysiology: Brainstem Auditory Evoked Potentials (BAEP), Electromyogram (EMG)

Others: Wound care, sutures, abscess incision/drainage (involving external and superficial abscesses not in the mouth/throat/neck area), fracture repositions, central venous line, insertion or removal of a chest tube.

Potentially: Blood test (arterial/venous/capillary), periferous venous access, arterial line, injections, bladder catheter, suprapubic catheter, stomach catheter, changing plaster mould.

\subsubsection{Fasting guidelines}

Most guidelines for PSA, including those of the American Society of Anaesthesiologists (ASA), use a 'nulla per os' duration of six hours for solid food and two hours for liquid food before a sedation in order to keep the risk of choking minimal. (Table 7)

Table 7: traditional ASA recommendations regarding having an empty stomach (American Society of Anaesthesiologists)

\begin{tabular}{ll}
\hline Food taken in & Duration of nulla per os \\
\hline Clear liquid & 2 hours \\
Breastfeeding & 4 hours \\
Milk powder & 6 hours \\
Non-human milk & 6 hours \\
Light meal & 6 hours \\
\hline
\end{tabular}

The ASA recommendations are congruous with those of the $\mathrm{CBO}$ guidelines on perioperative food (2007- http://www.cbo.nl/Downloads/304/rl_periovoed_07.pdf).

From information in literature, it appears that choking on stomach contents during PSA is extremely rare. There is no proof for the proposition that ensuring that the patient has an empty stomach actually reduces the risk of choking during PSA, or that not having an empty stomach increases the risk of choking. Furthermore, children with empty stomachs are often more difficult to sedate.

Since the stomach of some patients may be emptying at a slower rate in some circumstances or because the risk of reflux may be increased or choking may be induced by mask-andbag ventilation during hypoventilation, the working group has formulated the following recommendations:

\section{Recommendation 10}

1. Fasting is not needed for children undergoing light sedation

2. A child must preferably have an empty stomach for any (elective) PSA with moderate or deep sedation, in accordance with the same guidelines that apply to interventions taking place under general anaesthesia (two hours for clear liquids, four hours for breastfeeding, and six hours for other meals).

3. A child in an acute condition without an empty stomach is in itself no absolute contraindication for PSA. This is important if postponing the procedure would pose health risks and/or discomfort. However, in that case the choking risks must always be carefully considered, taking into account the choice of sedative, the depth of sedation, and any protection of the airway. In practice, this amounts to the following recommendations: 
(a) With PSA in an acute situation (without an empty stomach), deep sedation must be avoided as much as possible, since the protective airway reflexes may be disturbed or there is a high risk of respiratory impairment.

(b) If a procedure requires a form of deep sedation, the patient must have an empty stomach.

(c) If a procedure requiring a form of deep sedation is urgently needed and an empty stomach can therefore not be guaranteed, deep sedation must performed under the supervision of an anaesthesiologist in order to ensure optimal protection of the airway.

4. Not having an empty stomach must be no reason or excuse for performing a procedure with an ineffective form of light or moderate sedation.

\subsubsection{Informed consent}

According to the Medical Treatment Contracts Act (WGBO), patients must be informed of the procedure that they will undergo. Furthermore, the patient or their representative is entitled to know about the expected benefit of the diagnostic or surgical procedure, the risk of complications, and any available alternatives. The same applies in relation to PSA. The patient must grant consent, both for the procedure and for the proposed PSA, and the associated information must be stored in the medical file.

\section{Recommendation 11}

1. The patient/authorized representative must be verbally informed about the purpose, nature, and consequences of PSA, as well as any risks and alternatives for the proposed analgesic/sedation/anaesthesia/restraint.

2. The medical professional responsible for the PSA must also provide information to the child/authorized representative and obtain the consent of the child or authorized representative.

3. If the medical professional responsible for the PSA delegates the provision of information to a representative, it is recommended that written agreements are made about the content of the information to be provided.

4. It is recommended that a note is made in the patient records of which type of PSA consent was granted for, by who (child and/or authorized representative), to whom consent was granted, and when.

5. When information is provided to a foreign speaking patient and/or his parents/caretakers, neither the child itself nor another child should be used as interpreter

6. In addition to verbal information, it is recommended that written information is provided, including in any relevant foreign languages.

\subsection{Morbidity, mortality, safety, and registration}

During moderate and deep PSA and during the subsequent recovery phase, there is a small to real risk of complications which can only be recognized and treated if responsible professionals are present. The main complications that need to be recognized and treated quickly are hypoxia, hypoventilation, and/or obstruction of the airway. Some medicines are associated with hypotension, but the clinical relevance of this for patients who are otherwise healthy is doubtful. 
Although the safety profile of PSA medicines varies, the working group is of the opinion, based on the available scientific evidence, that the safety of PSA is primarily determined by the preconditions and professional competencies, rather than the pharmacological characteristics of the sedatives administered.

The main modern international guidelines indicate that in selected patients, sedation and/or analgesia (PSA) can be performed by non-anaesthesiologists under certain conditions. These conclusions are derived from data gathered from large surveys. The most frequent, significant complications are hypoxemia and hypotension. The most recent data nevertheless indicates that mortality and morbidity associated with PSA are very low, given sound monitoring and adequately trained staff. The same applies to the use of short-duration intravenous anaesthetics (e.g. ketamine, propofol) for PSA by trained non-anaesthesiologists.

It is imperative that PSA data are properly recorded in the Netherlands, as a basis for scientific research to establish the quality of a standardized sedation and/or analgesia (PSA) practice, and to improve it if required.

\section{Recommendation 12}

If the necessary quality and safety requirements are met, PSA, including deep sedation through short-duration intravenous anaesthetics (e.g. propofol), can be justifiably performed by a non-anaesthesiologist. The quality and safety requirements are described in the chapters "patient selection," "professional-dependent preconditions and competencies," "monitoring," and "means of resuscitation and emergency procedures." For these requirements, a distinction is made between:

(1) PSA that is limited to light sedation/anxiolysis.

(2) PSA for the purpose, or with the possibility, of resulting in moderate to deep sedation.

\section{Recommendation 13}

In order to obtain a better insight into the daily practice of PSA, the working group recommends that instances of morbidity and mortality resulting from PSA in the Netherlands are logged in a registration system.

\section{Recommendation 14}

The working group recommends that the scientific associations set up a national register for PSA. The scientific associations would play a coordinating role. It seems preferable that the national working group develops from the working group that has drawn up these guidelines and that it becomes responsible for:

1. Implementation.

2. Maintaining the guidelines.

3. Setting up a national registration system for PSA-related complications.

4. Supporting training. 


\subsection{Professional-dependent preconditions and competencies}

\section{GENERAL RECOMMENDATIONS}

\section{Recommendation 15}

\section{A. In relation to moderate and deep sedation}

1. Any PSA intended for or possibly leading to moderate or deep sedation must be carried out by an anaesthesiologist or by a specifically trained non-anaesthesiological professional with demonstrable competencies as described in recommendation 16.

2. The working group has established that there are currently few specialists in the Netherlands who are specifically trained in the practice of performing PSA in children. It is therefore recommended that the competencies in terms of skills and competencies in PSA are made more specific and that specific training is put in place (see chapter on training).

3. PSA in certain categories of children and risky interventions, as described in "1.2. Patient selection, the empty stomach requirement and informed consent," must only be performed under the direct supervision of an anaesthesiologist.

\section{B. In relation to light sedation/anxiolysis (nitrous oxide* or a one-off dose of midazolam**)}

1. Light sedation must be performed by a professional with demonstrable competencies in the field. The level of competence required in this respect is lower than for moderate to deep sedation (see recommendation 16). On that basis, light sedation can be carried out safely by a non-anaesthesiologist.

2. The working group notes that there is no training course in the Netherlands teaching light sedation, except in the field of specialist dentistry. Accordingly, the working group recommends that such training is arranged for all professionals who carry out (small) painful or frightening procedures in children (see chapter on training).

* to a maximum nitrous oxide concentration of $50 \%$

** $0.1 \mathrm{mg} / \mathrm{kg}$ intravenously or $0.2-0.5 \mathrm{mg} / \mathrm{kg}$ transmucosally

\section{NECESSARY COMPETENCIES}

There is a growing demand for effective PSA in the medical care of children. In general, it is fair to say that insufficient anaesthesiologists are available to meet that demand. However, solid scientific evidence shows that adequately trained non-anaesthesiologists are able to perform safe and effective PSA, including deep sedation with intravenous anaesthetics (see recommendation 12). Such a professional is referred to in this guideline as a "PSA practice specialist" (see also the chapter on training).

A PSA practice specialist can come from a variety of backgrounds: if available, child intensivists and neonatologists can request to take on a limited part of PSA, based on the competencies associated with their specific area of expertise. Conceivably, it can be very useful to let other medical disciplines (e.g. Emergency Care physicians) acquire competencies in PSA, or to train specific doctor's assistants in performing PSA in children.

In order to be able to perform high-quality and safe PSA for a large group of patients who need to undergo diagnostic or therapeutic interventions in a manner that is comfortable for them, a specialist in the practice of PSA must be able to screen patients, give them information and advice, and perform PSA independently (with, at the most, indirect supervision) in paediatric patients without, or with only few, general additional diseases (ASA I and II). Such a person must 
have knowledge of the various PSA techniques, the anatomy of the intervention area, specific pharmacological knowledge, the necessary knowledge to carry out adequate screening, monitoring knowledge, and have the necessary resuscitation skills.

The working group has formulated recommendations on the basis of its literature study in relation to the competencies that a specialist in PSA practice should be able to demonstrate.

Although it can be expected that anaesthesiologists already have most of these competencies at their fingertips, it must be emphasized that for PSA they also need to have competencies regarding child-centered preparation and circumstances, and the use of non-pharmacological techniques.

\section{Recommendation 16}

\section{A. Recommendations in relation to general competencies}

Any professional performing a PSA must have at least the following competencies, skills, or conditions, regardless of the medicine used for sedation and regardless of the intended sedation depth:

1. Knowledge of the indications, contra-indications, and requisite precautions for the sedation technique used, acquired through specific training or demonstrable relevant experience;

2. Regular experience with the applied medication or technique. The workgroup is of the opinion that "regular experience" must be interpreted as a minimum of 50 PSA sessions per year;

3. Knowledge to apply the form of PSA that is most appropriate for the procedure and the patient;

4. The ability to guarantee the optimally effective sedation level in a predictable manner. An effective sedation level is the sedation level required for the procedure to be successful and high-quality, while guaranteeing optimal comfort for the patient;

5. The ability to perform pre-procedural screening and a systematic risk analysis;

6. The ability to inform the patient, parent(s), or carer(s) about the PSA technique, the effects, potential adverse effects, and possible alternatives. The information must be given in time and be appropriate for the comprehension level of the patient and parent(s)/carer(s). The information must be given verbally and preferably also in writing;

7. The ability to guarantee child-centered circumstances before, during, and after the procedure;

8. The ability to apply or arrange for complementary non-pharmacological techniques to be used when possible (preparation, distraction, hypnosis, or self-hypnosis);

9. The ability to apply effective local or topical anaesthesia, when possible;

10. The ability to organize the necessary monitoring and rescue facilities during and after the procedure for as long as the consciousness level is lowered;

11. The ability to organize a supervised recovery phase and be able to identify the discharge criteria;

12. The ability to organize the immediate availability of a resuscitation team or a professional trained in Advanced Paediatric Life Support;

13. The ability to supervise, record, assess, and optimize the quality of the sedation in terms of safety and effectiveness. 


\section{B. Specific recommendations concerning competencies for moderate and deep sedation}

B.1.During a PSA with children involving (a risk of) moderate to deep sedation, the PSA must be carried out by a separate professional who is not involved in the actual intervention or operation.

B.2. During a PSA involving (a risk of) moderate or deep sedation and during the subsequent recovery, a professional must be present with the following necessary competencies, skills, and conditions:

1. The ability to assess and interpret the sedation depth;

2. The ability to guarantee the necessary monitoring of vital parameters and being able to appraise and interpret the monitored information (including capnography);

3. The acquired knowledge by means of a specific training course and the ability to manage at APLS* level as a result of repeated training (i.e. at least once a year):

- Techniques intended to guarantee an open airway;

- Techniques to administer mask/balloon ventilation;

- Administering antagonists;

- Cardiac compressions.

\section{Specific recommendations concerning competencies for light sedation/anxiolysis}

During a PSA involving light sedation and during the subsequent recovery phase, a professional must be present who still needs to have the following competencies, skills, and conditions:

1. The ability to assess and interpret the sedation depth;

2. To retain continuous verbal contact with the patient in the absence of any other form of monitoring;

3. The acquired knowledge by means of a specific training course and the ability to manage at BLS** level as a result of repeated training (i.e. at least once a year):

- Techniques intended to guarantee an open airway;

- Resuscitation techniques.

* APLS=Advanced Paediatric Life Support

**BLS=Basic Life Support

\subsection{Monitoring}

Serious cardiopulmonary complications with PSA are rare but not impossible. Cardiopulmonary (near) incidents nevertheless occur frequently to very frequently, depending on the procedure, the patient, and the definition. These incidents are usually brief and of a limited nature, but some of them are omens of a serious complication with permanent damage. It is therefore necessary to keep a close watch on the health status of the patient. The analysis of serious complications or fatal incidents in the context of a PSA teaches us that inadequate or absent monitoring is an important risk factor.

The working group has formulated recommendations about the monitoring required during PSA. A distinction is made between light sedation/anxiolysis on the one hand and moderate and deep sedation on the other hand (see recommendation 4 ) 


\section{Recommendation 17}

The recommendations formulated in relation to monitoring relate to the period during which consciousness is decreased. This is the case from the time the sedative is administered until the moment that it has completely worn off and the patient is fully awake again.

\section{PULSE OXYMETRY}

The most prevalent side-effect of PSA is hypoxia. It is therefore necessary to monitor patients earmarked for moderate to deep sedation continuously using a pulse oxymeter. The pulse oxymeter is a simple, non-invasive monitor that can detect a decrease in arterial oxygen saturation at an early stage, making it possible for corrective measures to be taken in time. However, the pulse oxymeter does not monitor the effectiveness of breathing. Based on its literature study, the working group has formulated the following recommendations:

\section{Recommendation 18}

1. For a PSA with light sedation/anxiolysis of a previously completely healthy patient, it may be decided not to carry out pulse oxymetry if monitoring takes place through continuous verbal communication.

2. For a PSA with (a possibility of) moderate to deep sedation:

- Oxygen saturation must always be monitored through pulse oxymetry.

- Pulse oxymetry must not be considered as a reliable monitor to assess the quality of breathing. In the absence of capnography, breathing must be monitored through continuous clinical assessment. The use of a pulse oxymeter must therefore not be a reason to decide against the continuous clinical assessment of respiratory quality by a professional who has this as their only task.

3. When using pulse oxymetry during a PSA for an MRI examination, suitable equipment must be used for this task.

\section{MONITORING OF THE AIRWAY AND THE BREATHING (VENTILATION)}

Respiratory depression is a rare but possible complication of PSA, particularly in moderate to deep sedation. It nearly always involves a certain level of hypoventilation (of which the most extreme form is apnea) and to a lesser extent a (partial) obstruction of the airway following a loss of muscle tone in the upper airway.

Breathing can be observed in several different ways:

- Continuous clinical observation;

- With an electronic breathing monitor, using ECG electrodes;

- With a precordial stethoscope, used by an observer to perform a continuous check of the respiratory sound;

- With capnography ( $\mathrm{ETCO}_{2}$ monitor), measuring the $\mathrm{CO}_{2}$ level in the air exhaled by the patient with a special device.

The airway is best assessed by continuous visual and especially auditory observation. An electronic breathing monitor is not suitable for detecting a threatened or blocked airway. A precordial stethoscope and capnography will nevertheless be able to do this to some extent.

Based on its literature study, the working group has formulated the following recommendations: 


\section{Recommendation 19}

1. The following applies to any form of PSA:

a. It is necessary to continuously monitor the quality of breathing (frequency, depth) and the airway.

b. Electronic monitoring (through thoracic electrodes) must be considered as a technique that primarily provides information about breathing frequency, but that is unreliable for checking the depth of breathing and the airway patency. Continuous visual and auditory monitoring of breathing and the airway are therefore essential during PSA, regardless of whether breathing movement is already being monitored electronically.

2. In the event of light sedation/ anxiolysis the quality of breathing (frequency, depth), must be evaluated on the basis of continuous verbal contact, and continuous direct observation of breathing movements and the airway. In addition, a precordially used stethoscope can be used if desired.

3. In the event of PSA with (a chance of) moderate or deep sedation, the quality of breathing and airway must be monitored using: (1) continuous direct observation of the airway and breathing (possibly supplemented with electronic monitoring) by a professional who is doing this as a main task; and (2) preferably also by means of capnography. As far as capnography is concerned, the working group recommends that this technique becomes available in any setting where PSA is performed with a (possibility of) moderate or deep sedation.

4. For a PSA during which continuous visual and auditory observation is impossible or unreliable (for example during an MRI investigation or during radiotherapy), the working group recommends monitoring the quality of breathing and the airway at all times using capnography.

\section{ECG MONITORING}

The most recent international guidelines recommend measuring heart frequency during moderate and deep sedation, using an ECG. There is no sound scientific evidence to support this recommendation.

Primary arrhytmia and arrhytmia secondary to an arrhytmogenic effect from analgesics or sedatives are very rare in childhood. In childhood, bradycardia and asystole are mostly the (relatively late) consequence of deep and/or long term preceding hypoxia. In that respect, an ECG monitor is low-sensitive to an impending catastrophe.

Heart frequency can usually also be measured with a saturation meter and is then derived from the frequency of the pulsatile plethysmographic signal. This signal is nevertheless sensitive to interference caused by movement of the sensor (for example, if the patient is restless), to the circulation being cut off (for example, for a blood pressure reading), to surrounding light, restricted circulation, or poor circulation. A heart frequency reading through an ECG signal is therefore more reliable and can also act as a check on the indications from the saturation meter. Based on these considerations, the working group has formulated the following tentative recommendation: 


\section{Recommendation 20}

1. In the event of light sedation/anxiolysis, it is not necessary to perform ECG monitoring, unless there are other reasons for monitoring the ECG.

2. In the event of a PSA with (a possibility of) moderate or deep sedation the ECG must be monitored, regardless of the medication used.

3. If ECG monitoring is required during PSA for an MRI examination, special electrodes must be used.

\section{BLOOD PRESSURE MEASUREMENT}

In addition to hypoxemia, hypotension is a frequently reported adverse effect of PSA. Hypotension is usually the direct result of a pharmacological effect generated by the sedatives administered. In this regard, ketamine is typically associated with hypertension. It is doubtful whether these blood pressure fluctuations are clinically relevant in an otherwise healthy patient. No solid evidence can be found in literature about the need to measure blood pressure during PSA. Furthermore, measuring blood pressure can be undesirably stressful in a lightly sedated patient.

However, most recent international guidelines recommend that the blood pressure is measured regularly during moderate and deep sedation. Based on its literature study, the working group has arrived at the following recommendations:

\section{Recommendation 21}

1. In the event of light sedation/anxiolysis it is not necessary to measure blood pressure.

2. In the event of PSA with (a possibility of) moderate or deep sedation blood pressure must be monitored through intermittent non-invasive measurements, particularly to indicate any potential direct effects of the PSA medication on blood pressure.

\section{SEDATION LEVEL}

Research shows that in the event non-titratable PSA medicines are used, it is virtually impossible to predict the effective sedation depth. Since unexpected deep sedation is associated with an increased risk of complications, it is useful to have access to a continuous or repetitive objective measure of sedation depth. A distinction can be made between observational techniques and techniques using technical instruments.

\section{Recommendation 22}

From the administration of a sedative medicine, the awareness level and the extent of its decrease must be measured in a way that is as objective and reproducible as possible. In that way:

1. Unintentional sedation can be discovered in time;

2. The sedation success of a specific sedation protocol can be measured;

3. The necessary observation time during recovery is determined on an individual basis, based on the verifiable return to normal consciousness.

It is preferable to use a validated measuring instrument for the purpose. The working group recommends that the University of Michigan Sedation Scale (UMSS, Malviya 2002) is used. During the recovery phase, supplementary use can be made of the Modified Maintenance of Wakefulness Test (MMWT, Malviya 2004). 


\section{Recommendation 23}

The working group advises against neuromonitoring using bispectral analysis (BIS) for assessing patients during moderate and deep sedation, because it does not have any added value.

\section{ROUTINE ADMINISTRATION OF OXYGEN}

This is one of the more controversial points. Proponents argue that administering oxygen results in less frequent occurrences of desaturation of the arterial blood; antagonists are of the opinion that it increases the risk of unrecognized hypercapnia due to insufficient respiration. When capnography is performed, the benefits of administering oxygen probably outweigh the postulated disadvantages. Hence the following recommendation:

\section{Recommendation 24}

- Oxygen does not need to be administered as a matter of routine, but it is definitely necessary upon the first signs of hypoxemia, i.e. if the oxygen saturation decreases to below $92 \%$ or by an amount greater than $5 \%$ of the starting value.

- Administering additional oxygen may conceal a respiratory deficiency. Additional attention must therefore be paid to respiratory quality.

\subsection{Emergency measures, recovery, discharge, and reporting}

\section{EMERGENCY MEASURES}

Serious morbidity or death as a result of administering PSA is unacceptable. The quality assessment and safety policy must limit the risk of complications. In the event a resuscitation situation arises nevertheless, it must be tackled instantly and optimally. Particularly at locations outside hospitals, a great deal of attention must be paid to these provisions, including adequate and quick referral to a hospital. The management of the organization is responsible for this (through the local PSA quality assessment committee).

\section{Recommendation 25}

It must be feasible to intervene adequately and quickly during PSA and the subsequent recovery period. This applies to expertise, as well as to equipment and medicines. It means that a professional trained in Life Support (for light sedation to a level of at least Basic Life Support (BLS); for moderate or deep sedation to a level of at least Advanced Paediatric Life Support $(A P L S))$ must be in the immediate vicinity and must have access to all the essential resources needed to support breathing and circulation.

\section{DISCHARGE CRITERIA}

After PSA, the patient must be continuously monitored, for a period comparable with that during PSA.

Immediately after the PSA and the associated procedure are terminated, there will be an imbalance between the (burden of) pain and the sedation level. Since the stimulus of the procedure has fallen away, the risk that the consciousness level will drop significantly after PSA, and the risk of respiratory depression, an obstruction of the airway, and hypotension are consider- 
able. Clinical observation, registration of respiration, blood pressure, pulse rate, and oxygen saturation must therefore continue.

Patient discharge must always occur based on clear and verifiable crite.

\section{Recommendation 26}

In the recovery phase, following on immediately from the PSA, the patient must be monitored in the same way (with personal assessment and instrumental monitoring) as during the PSA procedure.

\section{Recommendation 27: Recovery and Discharge}

The requirements that must be implemented for monitoring the recovery of consciousness and vital functions, particularly after deep sedation, are not essentially different from those for general anaesthesia. The same applies to the discharge criteria. The working group therefore recommends that the same criteria are used:

\section{Concerning monitoring during the recovery phase (recovery)}

- In the recovery phase, immediately after PSA, the patient must be supervised in the same way (always with personal as well as instrumental supervision) as during the PSA procedure, until the patient meets the discharge criteria (see below);

- During the recovery phase following a PSA with (a possibility of) moderate or deep sedation, a professional must be present, who meets the following competencies, skills, and conditions:

1. The ability to assess and interpret the sedation depth;

2. The ability to guarantee the necessary monitoring of vital parameters and be able to appraise and interpret the monitored information;

3. Being trained in the characteristics and adverse effects of the medication used;

4. Knowing and being in control (as a result of repetitive training) of those techniques (for example, as taught in the Advanced Life Support (ALS) course) that are aimed at guaranteeing an open airway, the performance of artificial respiration with a mask/balloon, and at treating a deep bradycardia or asystole.

- The observations and findings during the recovery period must be documented in writing. At a minimum, the following information must be recorded: the monitored vital parameters, the evolution of the patient's consciousness, adverse effects, and complications.

\section{Concerning the discharge criteria}

- Determining the moment of discharge must be considered as a formal medical decision that:

1. Is based on a number of verifiable criteria established in advance.

2. Must be documented in writing.

- Before the patient can be discharged, a number of conditions (objective criteria) must be met:

1. The patient must be uninterruptedly alert and orientated, i.e. have the same level of consciousness as before the PSA. 
2. The vital parameters of breathing and circulation must be normal.

3. The patient must be able to drink, not be sick, and have adequate analgesia.

- The discharge must not take place earlier than:

1. One hour after the last intravenous administering of sedative.

2. Two hours after any administration of antagonists.

- The patient must be accompanied on the way home or to the relevant ward by an adult who can call help if necessary.

- On being discharged, the patient or his/her parent(s)/carer(s) must receive a comprehensible document containing:

1. Any relevant instructions for developments after the PSA.

2. Explanation about any late adverse effects and how they should be dealt with

3. Any prescriptions and follow-up appointments.

4. A phone number to report complications and to obtain further advice (available 24/7).

\section{REPORTING}

PSA is a medical treatment in itself and an essential component of the treatment or examination procedure. It must therefore be recorded in a report. No specific literature has been found in relation to reporting. The guidelines from other countries, which were based on expert opinion and consensus, make adequate reporting an absolute requirement. Particularly any medication given, vital functions, the sedation level, the patient's reaction to the medication given and any adverse effects, and complications. It should also include the measurements of the sedation level and vital functions during postprocedural monitoring.

\section{Recommendation 28}

A careful report must be made of every PSA. This report must contain the information known prior to the PSA (risk analysis, informed consent, status in relation to empty stomach), a report of the procedure itself, and the development of the postprocedural period. The medication given and the reaction of the child to the medication, as well as monitoring data and postprocedural recommendations, must also be included.

\subsection{Non-pharmacological techniques}

PSA endeavors to make the child as comfortable as possible during a painful and/or stressful period. Sedatives and/or analgesics can be used for this purpose. However, it is certainly conceivable that the effect of the medication will benefit from the child and its parent(s) being well prepared for the procedure, taking into account specific, child-related circumstances, for which use is made of specific behavior-oriented and/or psychological techniques. In the guidelines on PSA from the American Academy of Paediatrics, it is stated that particularly with older and cooperative children, the use is recommended of non-pharmacological techniques such as distraction, the presence of the parent(s), certain imagination techniques, and hypnosis. Based on its literature study, the working group has formulated the following recommendations: 


\section{Recommendation 29}

1. According to the working group, non-pharmacological techniques must be considered as an indispensable part of PSA in children. Examples are:

- Giving information that is targeted at the child and at the parent(s). This means that honest information must be given at the level of the child and its parent(s). In this respect, it is recommended to no longer use the vague term "just a little nap." When giving "full information" to young children, it is important that the information is: (1) adjusted to their level of understanding; (2) adjusted to the manner in which the child thinks about the procedure or fears it; and (3) is also provided at sensory level (what will the child feel, see, hear, and smell) in order to make the event highly predictable for the child.

- Play-therapeutical preparation.

- Presence and involvement of the parent(s)*.

- Child-focused circumstances and professions.

- Applying distraction techniques.

- Using hypnosis.

* The presence of the parent(s) during the procedure can have positive as well as negative effects on the child's level of distress. Anxious parents can have a negative effect on the distress of a calm child. Calm parents can reduce the level of distress of an anxious child. There are indications that the presence of parents who have been specifically prepared and trained to give their child adequate support during the in duction for the anaesthesia lead to less distress and postoperative pain in the child.

2. Knowing and controlling or being able to delegate non-pharmacological techniques must be an essential competence for a professional who is responsible for PSA in children.

3. For children with learning difficulties, a careful check must be made of which procedures would benefit from PSA and which must be carried out under general anaesthesia. It is important to evaluate the level of the child's cognitive and emotional functions as accurately as possible in order to be able to use appropriate non-pharmacological techniques. Parents/companions know the child best and their information or experience is therefore invaluable.

\subsection{Swaddling, immobilization, and restraint during procedures}

The physical fixation and/or immobilization of children during a diagnostic or therapeutic procedure form part of the daily practice of providing healthcare to children. The following are urgent reasons to proceed to restraining and immobilizing children:

- [The need] to overpower the child's resistance to a procedure which is necessary, but experienced as painful and/or frightening by the child, to enable the procedure to take place.

- The partial or complete failure of a child to cooperate with procedures due to a lack of understanding or susceptibility to being distracted, making it impossible to give an explanation or to obtain consent for a necessary medical intervention to be performed.

$=$ Not having or not using effective analgosedation. 
The working party considers that specific recommendations are necessary, since carrying out procedural restraint in children could be used as an excuse for ineffective PSA and also because it gives rise to important legal and ethical questions.

In order to avoid any ambiguity, the working group has defined the following categories of fixation on the basis of its literature study:

1. First, swaddling. Although it has only been studied in the context of soothing crying babies, the technique is also used for specific procedures with young infants when prolonged immobilization is required (for example, for an MRI scan). The objective of swaddling is to induce comfort, relaxation, and sleep without using restraining force.

2. Second, immobilizing or holding. This is when a professional (or a parent) helps a child to lie still or to keep a limb as motionless as possible without using (significant) force. When this happens with the consent of the parent(s) and the (apparent) agreement of the child, it constitutes informed consent and everyone (including the child and its parent(s)) agrees that the immobility is necessary for the procedure to succeed.

3. Last, restraint or clinical holding. This is when the child's willpower and resistance are broken, using force. The child is forced to undergo a procedure without its agreement (up to the age of 12) or consent (from the age of 12). Sometimes, a further distinction is made between passive and active restraint. With passive restraint, the child is kept under control using a harness or straightjacket-like device, a sheet, or restraining ribbons. Active restraint refers to a professional or the child's parent(s)/carer(s) manually restraining the child.

\section{SWADDLING}

Various recent guidelines recommend the use of swaddling (possibly including a preprocedural feed) in neonates who need to undergo a radiological examination. No scientific research has been carried out into the effectiveness and safety of this technique. Nor has any research ever studied the combination of a preprocedural feed and/or swaddling with a sedative. It is accepted that both the preprocedural feed and swaddling may increase the risks of a PSA since (1) a child with a full stomach is moderately to fully sedated, (2) the visual observation of the child is impeded, and (3) the child is forced into a position that may counteract the airway remaining open as well as important protective reflexes (such as vomiting). Based on its literature study, the working group has formulated the following recommendations:

\section{Recommendation 30}

With young infants (under six months old) swaddling on its own or in combination with a feed immediately before the procedure is considered as an alternative to sedation for painless procedures. Swaddling must therefore be considered as an age-appropriate, nonpharmacological manner in which to create optimal comfort during a procedure.

The procedure is preferably planned immediately after an already existing feeding time, which means that the normal feeding pattern of the child is not compromised.

Swaddling and preprocedural feeds must not be combined with a sedative.

\section{FORCED IMMOBILIZATION AND RESTRAINT}

There has been little scientific research into this subject, and it only consists of a few epidemiological studies, the opinions and experiences of carers and parents, and the expert opinions of 
professionals. One recent guideline expresses a view on the subject, stating that restraint is in principle not acceptable for procedures that are not life-saving (SIGN guideline 2004).

The working group has also used ethical and legal considerations, the latter based on national and international legislation, to arrive at the following recommendations:

\section{Recommendation 31}

1. The forced immobilization and restraint of a child against its will for the purpose of overcoming the child's resistance to non-vital, non-acute, but medically necessary health treatment is in principle unacceptable.

2. The working group is of the opinion that it is incorrect to interpret the Medical Treatment Contracts Act in a way that seemingly condones the point of view that the resistance of a child under twelve years old can be ignored provided the parent(s) consent to the child being immobilized or restrained.

3. The working group feels that the professional responsible for performing a painful or stressful intervention on a child, is responsible for guaranteeing the child's optimal comfort and that the person concerned is in principle not allowed to use physical restraint or immobilization. The only way in which these procedures can be deployed is as part of an individual approach and a protocol that aims for maximum comfort, in the working group's opinion. Depending on the individual needs of the child, the protocol must be able to offer emotional support, hypnosis, distraction techniques, local anaesthesia, PSA, and/or general anaesthesia.

4. Immobilizing and restraining a child in the context of an effective, non-life saving medical procedure is only acceptable if:

(1) it does not use force;

(2) it is aimed at helping the child to keep its body or part of its body still, or for safety reasons;

(3) informed consent has been obtained*;

(4) everyone concerned (including the child and its parents) is convinced that the immobility is necessary for the procedure to succeed, and;

(5) the procedure and immobilization are interrupted if it appears form the child's verbal or non-verbal communication that it expresses resistance to the procedure.

* the working group judges that the professional responsible for a painful or stressful procedure with a child has a duty to explain the level of distress and pain to the child and parent(s), and to present the child and parent(s) with alternative methods for immobilization or restraint (such as local anaesthesia, PSA, and anaesthesia). See recommendations on "informed consent."

5. In the event of a life-saving or highly urgent medical procedure, restraint and/or immobilization may be appropriate because the condition of the patient prevents the use of a better alternative or because the alternative (for example, PSA) may be dangerous to the child.

6. The PSA of a child used in combination with passive or active restraint, or immobilization in order to keep the child under control and/or to enable the procedure to be performed, must be considered as an ineffective and unacceptable form of PSA.

7. For children with learning difficulties and other children who are more difficult to prepare for actions or procedures, and who are not or are barely cooperating, the procedures must preferably take place under general anaesthesia, provided no easier alternatives are available. 
8. It is necessary to develop a clear legal, ethical, and practical framework for the use of restraint and immobilization, in order to help the professional establish more clarity about (1) his/her responsibility in these procedures and (2) the manner in which immobilization and restraint (if unavoidable) can be correctly administered without neglecting the individual needs of the child.

\subsection{Recommendations for the use of medication cocktails for PSA in children}

A cocktail is a combination of medicines that are administered in fixed proportions. Several cocktails of sedatives, analgesics, and/or medicines with a sedating side effect are described and used in the context of PSA. The most frequently used mixture is the lytic (or MPC) cocktail; composed of meperidine, chlorpromazine, and promethazine. All three are medicines with a sustained effect, of which the individual pharmacological properties in children have been barely investigated. The medication is administered intramuscularly. Sometimes, parts of the cocktail are combined with chloral hydrate or a benzodiazepine.

Cocktails are still frequently used in paediatrics in the Netherlands, particularly for PSA during imaging (e.g. MRI scanning).

There is little recent scientific research on the cocktails used for PSA. Most recent international guidelines advise against using cocktails. The main arguments are: an immense disparity in when they start to take effect and for how long they work, limited effectiveness, the risk of oversedation, and the association between the risk of incidents and the use of several products simultaneously.

As far as the MPC cocktail for MRI examinations is concerned, the very limited literature on the subject is inconclusive with regard to effectiveness and safety.

\section{Recommendation 32}

The working group advises against sedation with an intramuscular cocktail, because there are better alternatives in terms of comfort, safety, and effectiveness. These alternatives must be readily available.

\subsection{Recommendations for the use of nitrous oxide (mixtures) for PSA in children}

From recent. high-quality scientific research, it appears that the inhalation of nitrous oxide (compound) is both a very safe and an extremely effective PSA technique for minor, but nevertheless painful and/or frightening procedures in children. Its effectiveness is further increased in combination with a local anaesthetic and a non-pharmacological technique to optimize the cooperation of the patient during inhalation.

Particularly in the context of short-term procedures, such as blood tests, inserting an intravenous catheter, bone marrow and lumbar punctures, wound care, suturing cuts, and repositioning uncomplicated fractures, nitrous oxide can make an important contribution to achieving optimal PSA. These procedures are currently often carried out without any form of PSA or only under local anaesthesia (for example Emla ${ }^{\circledR}$ ) or by using midazolam, intended for anxiolysis and amnesia. For those reasons, these interventions are often (very) traumatizing for children and it is frequently necessary to restrain and/or immobilize them. 
Nitrous oxide (up to $50 \%$ ) causes only light sedation that disappears quickly after the administration is discontinued. The level of required preconditions and competencies is therefore lower than for PSA techniques causing moderate to deep sedation. From the perspective of patient safety, nitrous oxide can therefore be readily used. Only a very brief recovery period is required. Lastly, nitrous oxide has the advantage that it can be administered without the need for intravenous access.

In hospitals worldwide, nitrous oxide is used very frequently and virtually as a matter of routine for the aforementioned procedures.

Since 2004, the use of nitrous oxide has become quite controversial in the Netherlands, in terms of the possible risks of toxic effects for the staff administering it (namely: toxicity to an unborn child and effects on fertility). The working group has therefore carefully studied the literature with regard to potential toxic effects

Observation: These guidelines recommend the use of nitrous oxide for PSA in children. This stance is at variance with the recent tendency in the Netherlands to ban any treatment with nitrous oxide. We therefore invite readers to study the literature survey in the original guideline text and the considerations following the recommendations below.

Based on its literature study, the working group has arrived at the following recommendations:

\section{Recommendation 33}

1. It is recommended that nitrous oxide is available as a PSA technique for use in children (over one year old) when it can be expected that they will put up strong resistance to, or suffer great discomfort from, a relatively minor but nevertheless painful or stressful procedure (for example, blood test, insertion of a drip, lumbar puncture, other punctures, bladder catheterization, the installation of a stomach tube, suturing or dressing wounds, fracture reductions, and small surgical procedures). After all, this form of PSA is possibly more effective than the use of local anaesthesia and/or anxiolysis with midazolam. The need for forced immobilization and restraint for these procedures may even be reduced as a result. The following additional benefits must also be taken into account: (1) the fact that no intravenous access is needed, and (2) the very brief recovery phase, enabling a quick discharge.

2. Contraindications for using nitrous oxide are: vitamin B12 deficiency, methionine synthase deficiency, methylmalonic acidemia, homocystinuria, (the possibility of) pneumothorax, and (the possibility of) intracranial air. These must be considered as contraindications for the use of nitrous oxide.

3. The use of nitrous oxide is not recommended for refractory or uncooperative patients. After all, having the mask forcefully imposed is traumatizing and leads to the workplace being contaminated with nitrous oxide.

4. In order to achieve maximum efficiency, it is necessary to:

- Combine nitrous oxide with local anaesthesia and with behavior-oriented, nonpharmacological techniques;

- Properly inform and prepare the child, in order to optimize acceptance of the mask and the procedure.

5. Administering nitrous oxide requires that the professional working with it is trained and 
takes all the necessary precautions to minimize the area being contaminated, in conformity with the applicable health and safety regulations. Based on its literature study, the working group is of the opinion that nitrous oxide does not pose a significant danger to the professionals working with it, provided adequate scavenging and ventilation is in operation.

6. If nitrous oxide is used for PSA in children, it is recommended that a special nitrous oxide device is used, with a continuous flow and measurable concentrations of nitrous oxide and oxygen. After all, the technique offers two major advantages in comparison with equimolar mixtures:

- The dose can be gradually increased and optimally titrated;

- The device also takes care of the extraction of excess gas.

7. Nitrous oxide up to $50 \%$ and without being combined with other sedatives only causes light sedation. It is therefore sufficient to monitor the patient by means of continuous observation and a saturation meter.

8. After the inhalation of nitrous oxide is stopped, the nitrous oxide leaves the body through the lungs. For that reason, there is a theoretical possibility of redistributive hypoxia in the event that nitrous oxide completely fills up the alveoli and prevents oxygen intake. It is therefore recommended to let the patient inhale 100\% oxygen for several ( 3 - 5) minutes after nitrous oxide inhalation.

\section{Part 2 Procedure for specific recommendations}

2.1. General recommendations for any procedure involving the administration of PSA

Part 2 of the guidelines contain recommendations for a number of procedures concerning the use of the most optimal PSA techniques in children. These recommendations are each time based on careful literature study and on the considerations of the working group itself.

The definition of what must be considered as optimal PSA will act as general guidance for each procedure. For that reason, Recommendations $\mathbf{2}$ and $\mathbf{3}$ of these guidelines apply to every procedure for which PSA is considered or applied:

\section{Recommendation 2}

In all cases, it is necessary to opt for the most effective PSA technique:

1. The nature of the procedure to be performed, the individual sedation requirement of the patient, and the patient's state of health determine which sedation level and which PSA technique (sedation and/or analgesia) is most appropriate to make the intervention as successful as possible and in a manner that is as comfortable and safe as possible for the individual patient. Optimally successful means that the PSA technique aims for a $100 \%$ predictable procedural success and timing, high predictability of the sedation level and duration, and minimal induction and recovery times. Optimally comfortable means minimal to absent pain and anxiety for the patient, and the absence of any need for restraint.

2. For (very) painful and/or (very) stressful procedures and for procedures in young children that require prolonged immobility or cooperation, deep sedation may be required.

3. Effective systemic and/or topical analgesics must be used on every occasion for painful 
procedures. Using only sedation for painful procedures is not acceptable, even if it in the expectation that the sedative will have an anterograde amnesic effect.

4. The aforementioned aspects (i.e. intended sedation level, applied sedation technique, individual patient characteristics, and state of health) determine the necessary preconditions and the necessary competencies of the medical staff involved (see the specific recommendations in the chapters on competencies, monitoring, makeshift facilities, and training).

\footnotetext{
A Point 1 of this recommendation is not immediately fully achievable. It can be expected that professionals will consider the ratified guideline as an immediate cause to develop initiatives that will help achieve this recommendation within the foreseeable future.
}

\section{Recommendation 3}

For the optimal procedural comfort in children, it is not sufficient for an optimal PSA technique to be available and deployable. Prevention of painful/stressful procedures, banishing securing and restraint, optimal local/topical anaesthetics, non-pharmacological techniques, and rescue anaesthetics must also belong to a comprehensive policy aimed at safe and comfortable care.

\subsection{Endoscopic procedures*}

\section{GASTROINTESTINAL ENDOSCOPIES}

Gastrointestinal endoscopies are frequently used and necessary in diagnostic, and sometimes therapeutic, procedures in children with gastrointestinal complaints. Endoscopies are uncomfortable, stressful, and moderately painful procedures and they can therefore not usually be carried out without sedation or anaesthesia in children, even if they have been suitably prepared.

Based on its literature study, the working group has therefore formulated the following recommendation:

\section{Recommendation 34}

1. A gastrointestinal (GI) endoscopic examination in a child must be carried out in principle under general anaesthetic or deep sedation. If it is decided to opt for deep sedation, then titratable medicines must be used that are certain to lead to an effective level of deep sedation. Of all the medicines studied, propofol is the most effective, if necessary in combination with midazolam or an opiate.

2. The working group advises against the following forms of PSA for GI endoscopic examinations:

- Using ketamine for endoscopic examinations of the esophagus, stomach, and duodenum, since there is an increased risk of laryngospasm.

- Using a benzodiazepine on its own or the combination of benzodiazepine with an opiate. Both forms of PSA are substantially less effective than anaesthesia or deep

\footnotetext{
* See also: 2.1. General recommendations for any procedure involving the administration of PSA.
} 
sedation with propofol.

- Benzodiazepines must not be considered as suitable medicines to generate a reliable level of amnesia for endoscopic procedures.

3. As far as rectoscopies are concerned, it is worth contemplating whether the investigation could be carried out without PSA insofar as informed consent has been obtained and provided the child is not scared or opposed to the examination.

4. If general anaesthesia or the support of an anaesthesiologist are not feasible, an endoscopic department must have access to the logistic possibilities as well as trained professionals in order to provide safe and effective deep sedation that fulfils the preconditions of these guidelines.

5. Premedication with midazolam taken orally can be considered prior to deep sedation. It reduces stress levels for inserting the drip at the start of the procedure and may therefore result in a smaller dose of propofol being required.

\section{FLEXIBLE BRONCHOSCOPY}

Bronchoscopies are uncomfortable, stressful, and moderately painful procedures and they can therefore not be carried out without sedation or anaesthesia in children, even if they have been suitably prepared. Most children that need to undergo a broncoscopy (whether or not in combination with a bronchoalveolar lavage) have an underlying pulmonary problem and/or a compromised airway that makes them even more vulnerable to PSA and procedure-related complications.

Based on its literature study, the working group has therefore formulated the following recommendation:

\section{Recommendation 35}

Bronchoscopies in children are only comfortable and safe if they are performed:

- Under anaesthesia or under a manageable form of deep sedation during which full control is kept over whether breathing is spontaneous;

- Under the supervision of an anaesthesiologist. A paediatric intensivist can be responsible for the PSA of intubated children admitted to an intensive care unit.

\subsection{Oncological procedures*}

Oncological children are frequently exposed to painful invasive procedures for diagnostics and therapy. Examples are taking blood using venipuncture, inserting a drip, inserting a catheter for a port-a-cath ${ }^{\circledast}$, bone marrow puncture, lumbar puncture, and sometimes a bone biopsy They have a high repeat rate and continue for years, particularly for patients with hemato-oncological disorders. A significant proportion of the patients and their parent(s) experience this period as stressful, which is correlated to the intensity of the treatment. Based on its literature study, the working group has therefore formulated the following recommendations:

\footnotetext{
* See also: 2.1. General recommendations for any procedure involving the administration of PSA.
} 


\section{Recommendation 36}

1. Given the existing indications are that experiences of pain contribute to negative late psychological effects from treatment in the survivors of childhood cancer, maximum investment must be made during the treatment in effective procedural pain and anxiety control, taking into account the individual wishes and needs of the patient.

2. Painful paediatric oncological procedures (such as lumbar puncture, bone marrow puncture, and bone biopsy) must preferably take place under general anaesthesia or under a deep PSA. If it is decided to opt for deep PSA, it is preferred to opt for powerful medicines that are highly effective (such as propofol, with or without an opiate, or ketamine), to prevent the need for particularly rigid restraint or immobilization of the patient. It is preferable not to use PSA with benzodiazepine, whether or not in combination with local anaesthesia, since optimal comfort cannot be guaranteed in most cases.

3. It is possible to deviate from this recommendation in individual cases, when the procedure is performed under local anaesthesia, possibly supplemented by a light form of sedation (for example benzodiazepine or nitrous oxide). In this case, it is nevertheless necessary that:

a. Full, informed consent has been obtained from the patient and that the latter prefers this working method;

b. No use is made during the procedure of forceful immobilization or restraint.

4. Local anaesthesia (in the form of Emla ${ }^{\circledR}$ or Rapydan ${ }^{\circledR}$, for example) must be administered to anaesthetize the skin when a port-a-cath ${ }^{\circledR}$ is inserted.

5. Practical or logistical arguments should be no reason for a child not to have access to deep PSA or general anaesthesia in the context of an oncological procedure. A paediatric oncological ward must therefore always have access to logistical facilities and trained professionals to provide safe and effective deep sedation that meets the preconditions set out elsewhere in these guidelines.

\subsection{Radiological procedures*}

The development and application of advanced radiological techniques such as computer tomographic examination (CT scan) and particularly magnetic resonance (MRI scan) in paediatrics have led to a surge in the need for PSA in radiology departments. Moreover, these diagnostic techniques are being used increasingly frequently, and at an increasingly young age. Immobilization must be ensured for a sufficiently long period, particularly for an MRI scan, to be able to generate high-quality images and to maximize the diagnostic possibilities. Children who are not, or are minimally, cooperative as a result of their developmental age can only undergo these examinations under some form of PSA. Furthermore, an MRI examination is very noisy (up to 100 decibels), which means that the sleep required during investigation must be sufficiently deep. Other radiological examinations, such as a micturating cystograms, are often too stressful for some children to undergo without PSA.

Based on its literature study, the working group has therefore formulated the following recommendations:

\footnotetext{
* See also: 2.1. General recommendations for any procedure involving the administration of PSA.
} 


\section{Recommendation 37}

Some form of PSA or general anaesthesia is recommended for any examination with imaging for which:

1. Children need to lie completely still for several minutes and for which it is expected that preparation, information, or distraction will not be enough to achieve that goal.

2. The examination is expected to generate a certain level of pain or stress, which cannot be adequately suppressed in any other way except through the use of PSA.

\section{RELATIONSHIP BETWEEN PSA TECHNIQUE AND (DEVELOPMENTAL) AGE}

\section{Recommendation 38}

1. Children who are insufficiently cooperative as a result of their (developmental) age and despite non-pharmacological techniques (for example play therapy) for a radiological examination, have the right to undergo the examination either under anaesthesia or under a form of PSA that is as safe and effective as possible.

2. In order to assess the chance of success and the risk of complications of a PSA in the context of a radiological examination, the (developmental) age must be formally taken into account as an important factor. Crucially, it must be borne in mind that children under one year old have a higher risk of complications, and that children aged over five have a greater need for deep sedation.

3. Feeding and/or swaddling may be a first choice for any necessary immobility and to obtain cooperation for the purpose of a radiological examination in infants (under six months old).

4. Children with learning difficulties and/or motor disabilities must receive an individually adjusted PSA that usually requires specific competencies of the professional, since these children (1) are often harder to sedate and (2) have a higher risk of complications. A PSA in these children must therefore preferably be performed under anaesthesiological supervision. The PSA and the recovery must always take place under anaesthesiological supervision in the event:

- of any anatomic or functional abnormalities of the airway and/or breathing (for example, muscular weakness, disturbed swallowing function, brainstem deviations, serious scoliosis);

- the intellectual limitation is such that it complicates an objective assessment of consciousness, sedation depth, and recovery;

- it can be expected that high doses of sedatives may be required.

\section{PSA TECHNIQUES FOR THE RADIOLOGICAL EXAMINATION OF CHILDREN}

\section{Recommendation 39}

1. With an ELECTIVE radiological examination that can be expected to be unsuccessful or uncomfortable for the child if it is carried out without sedation or with light sedation/anxiolysis, it is preferable to opt for a maximal titratable and controllable form of 
deep sedation (e.g. with propofol) or general anaesthesia because these techniques guarantee the highest level of effectiveness.

2. A radiology department performing painful, stress-inducing, or immobility-dependent radiological examinations in children must have access to the logistical possibilities and trained professionals to provide safe and effective deep sedations that comply with the preconditions set out elsewhere in these guidelines. Since the radiology department is pre-eminently a place with limited opportunity to observe and access the patient, and it must be possible to intervene quickly and adequately, the examinations should preferably be clustered and completely carried out by a specialist in PSA practice or by an anaesthesiologist. Moreover, the MRI department needs adjusted anaesthesiological equipment and materials. The working group emphasizes that the logistics must be of such a nature that the above resources must also be readily accessible and available with minimum delay.

3. If no anaesthesia or deep sedation is available for an ELECTIVE radiological examination, a decision can be made to use non-titratable sedatives.

a. The working group is nevertheless of the opinion that the systematic use of nontitratable sedatives must be discouraged, because the effectiveness of these medicines is always lesser than with general anaesthesia or deep sedation with titratable medicines. In particular, control over the starting time of an effective sedation level, and hence the synchronization with the anticipated time of the examination, are fairly poor. Putting together and using a procedural sedation team that can follow an individually developed sedation protocol (individual choice of medication, optimal comfort, individual timing, low-stimulus environment) is a long-winded and laborintensive method that can nevertheless lead to a higher success rate with these medicines.

b. Non-titratable sedatives must be considered not as light sedatives, but as medicines that are capable of generating moderate to deep sedation. Using these sedatives therefore requires the same preconditions as those applicable to deep sedation: specific monitoring, competencies, skills, and conditions as set out in part I of these guidelines.

c. Of all non-titratable sedatives studied (midazolam, chloral hydrate, pentobarbital and lytic cocktails), pentobarbital and chloral hydrate appear to be the most effective and safe. Their effectiveness is highest for children of a young age (under two years old)

d. The use of lytic cocktails or combinations of sedatives (for example, midazolam together with opiates) is strongly discouraged because there are better alternatives in terms of comfort, safety, and effectiveness.

4. In the event a form of sedation is needed for an URGENT radiological examination, the sedation must be performed under the supervision of an anaesthesiologist or an intensivist, given the potentially serious condition of the patient and the absence of an empty stomach. 


\subsection{Neurophysiological procedures*}

Based on its literature study, the working group has formulated the following recommendations:

\section{Recommendation 40}

1. The circumstances of the examination and the preparation of a child and parent(s) for any form of neurophysiological examination must be optimal, in order to reduce the need for PSA.

2. EMG examinations are painful and can therefore not be carried out without restraint. For that reason, PSA, local anaesthesia (for example Emla ${ }^{\circledR}$ ), and non-pharmacological techniques will often be needed in addition to the aforementioned conditions. An individual decision will need to be made on the use of anxiolysis/light sedation or moderate/deep sedation. The desirability of PSA must nevertheless be weighed up against the potentially negative influence of the sedatives on the reliability of the EMG examination.

\subsection{Specific recommendations for PSA in children in an emergency*}

Accident and Emergency Departments (A\&E) and paediatric wards regularly have to deal with children in circumstances when a diagnostic or therapeutic procedure must be performed at (some) speed. Many of these procedures are painful and/or stressful. Common characteristics of these procedures are that: (1) they are (relatively) urgent; (2) they largely need to be carried out in children that do not have an empty stomach; (3) they are usually relatively limited in scope or complexity and; (4) they do not usually need to be carried out in an operating theatre. As a result of these characteristics, it is usually not possible to call on an anaesthesiologist.

In relevant literature, many non-anaesthesiological methods are described for carrying out these interventions as painlessly and with as little stress as possible. However, it appears that too little use is made of these methods, particularly in children. (MacLean, 2007).

The working group has subdivided the results of its literature study into two sections: first, we will discuss the use and suitability of various medicines. The medicines we will look at are: ketamine, midazolam, fentanyl, chloral hydrate, and propofol. Second, we will formulate recommendations for specific interventions in which medicines can be used in order to make the intervention concerned as free of pain and stress as possible.

\subsubsection{Medicines for PSA in emergencies}

\section{CHLORAL HYDRATE}

Based on its literature study, the working group has formulated the following recommendations:

\section{Recommendation 41}

1. The use of chloral hydrate must be limited to less urgent interventions because the sedation peak only occurs after 30 minutes, it leads to a long recovery period, and the sedation is not always sufficient.

\footnotetext{
* See also: 2.1. General recommendations for any procedure involving the administration of PSA.
} 
2. Chloral hydrate can be applied for sedation in the event of interventions that cause little or no pain (e.g. imaging).

3. It is not recommended to use chloral hydrate in children under six months old.

4. Chloral hydrate causes moderate to deep sedation. The appropriate preconditions on patient selection, skills, competencies, monitoring, and other preconditions set out in part I of these guidelines must therefore be complied with.

\section{MIDAZOLAM AND MIDAZOLAM+FENTANYL}

Based on its literature study, the working group has formulated the following recommendations:

\section{Recommendation 42}

The combination of midazolam + fentanyl intravenously is suitable for application in (urgent) painful procedures in children, provided the following recommendations are observed:

1. In the event fentanyl is used intravenously in combination with midazolam intravenously, fentanyl must be administered first, after which midazolam must be titrated to the required level of sedation, in order to keep the risk of respiratory depression as low as possible.

2. The combination of fentanyl i.v. and midazolam i.v. leads to moderate to deep sedation, which means there is an increased risk of respiratory depression. The associated preconditions in relation to the patient selection, skills, competencies, monitoring, and other preconditions set out in part I of this guideline apply in that case.

3. With PSA using midazolam and/or fentanyl, the antagonists of both medicines (flumazenil and naloxone respectively) must be close to hand in order to quickly treat any adverse effects of these medicines.

The following recommendations apply to the use of midazolam:

1. The working group is of the opinion that one standard dose of midazolam only causes light sedation/anxiolysis. Used in higher doses, and/or in combination with other sedatives, midazolam will cause moderate to even deep sedation in some patients. The respective preconditions in relation to patient selection, skills, competencies, monitoring, and other preconditions set out in part I of these guidelines will apply in that case.

2. It is not acceptable to use midazolam on its own for painful procedures, even if it is in the hopeful anticipation that the substance will have an amnesic effect. For painful procedures, midazolam must always be combined with an effective systemic or local/topical analgesic.

3. In the event midazolam is not administered intravenously, it must preferably be used sublingually, in order to prevent irritation of the nose in the event of intranasal administration, ineffective sedation in the event of rectal administration, or an unpredictable plasma concentration in the event of oral administration.

4. The non-intravenous use of midazolam is best used on its own for less urgent applications, in view of the fact that peek sedation only occurs after ten to thirty minutes and the sedation is not always sufficient.

Based on its literature study, the working group has formulated the following recommendations: 


\section{Recommendation 43}

Ketamine is suitable for application in (urgent) painful procedures in children, provided the following recommendations are observed:

1. Ketamine causes deep sedation to anaesthesia. Despite the retention of airway reflexes, airway complications and respiratory complications may occur in the form of vomiting, laryngospasm, respiratory depression, and apnea. If it is decided to use ketamine, it is therefore important to ensure that the preconditions in terms of patient selection, skills, competencies, monitoring, and the other preconditions set out in part I of these guidelines are met.

2. It is preferable to use intravenous ketamine if intravenous access is present or if it can be inserted quickly and with little (emotional) stress to the child. In other situations, ketamine can also be considered for intramuscular (i.m.) use, but in that case, intravenous access must be generated as quickly as possible. The comparatively quick recovery from ketamine i.v. in comparison with i.m. provides an advantage for short procedures such as repositioning and brief, painful wound dressing.

3. Although limited research has been carried out into the use of administering anticholinergic agents with ketamine, the working group recommends it for higher doses, in order to reduce hypersalivation. In the event atropine is used, we recommend $0.01 \mathrm{mg} / \mathrm{kg}$ (minimum $0.1 \mathrm{mg}$, maximum $0.5 \mathrm{mg}$ ). The anticholinergic agent can be administered intravenously before the ketamine, or simultaneously intramuscularly.

4. Based on the scientific evidence found, it is not recommended that midazolam is added to ketamine, in order to prevent any agitation at the time of recovery. Furthermore, the combination of ketamine and midazolam poses a higher risk of respiratory complications. Midazolam can nevertheless be administered in the event of agitation during the recovery period.

5. Contraindications for the use of ketamine are:

Absolute contraindications:

- the patient being under three months old

- known or suspected psychosis, even if the condition is stable or under treatment at the time of sedation

Relative contraindications:

- age between three months and twelve months

- procedures that may stimulate the posterior pharynx and interventions during which laryngeal stimulation may occur

- an active airway infection, including upper airway infections or asthma

- a known cardiovascular disorder, including angina, heart failure, or hypertension

- cranial trauma with a loss of consciousness, reduced consciousness, or vomiting

- tumors or disorders of the central nervous system or hydrocephalus

- glaucoma or acute eyeball trauma

- porphyria, thyroid gland disorders, or thyroid gland medication

6. In view of the risk of agitation during recovery, it is recommended that patients undergoing PSA with ketamine are allowed to recover in a very quiet room.

\section{PROPOFOL}

Based on its literature study, the working group has formulated the following recommendations: 


\section{Recommendation 44}

Propofol is suitable for application in (urgent) painful procedures in children. Propofol causes deep sedation to anaesthesia. The preconditions on patient selection, skills, competencies, monitoring, and the other preconditions set out in part I of this guideline must therefore be complied with. Since propofol is a fast-acting, very potent medicine that can quickly lead to oversedation and respiratory depression in untrained hands, the working group also has the following recommendations:

1. The person who performs the PSA must never be the same person as the one carrying out the procedure or intervention.

2. The PSA is preferably carried out by an anaesthesiologist.

3. If the PSA with propofol is carried out by a non-anaesthesiologist, it must be performed by a physician who has already been working with the medicine for a longer period of time and who is able to assess and deal with any respiratory complications.

4. PSA with propofol in patients of ASA class III or higher must be performed by an anaesthesiologist.

5. Pre-oxygenation and monitoring through capnography with PSA using propofol is strongly encouraged in order to restrict the comparatively high risk of respiratory complications.

\section{NITROUS OXIDE}

\section{See Recommendation 33}

Specific recommendations in relation to the choice of medicine for emergency procedures*

\section{VERY PAINFUL, STRESSFUL, AND/OR MORE COMPLEX PROCEDURES}

These interventions have in common that the combination of local/topical anaesthesia, light sedation/anxiolysis (e.g. midazolam or nitrous oxide), and non-pharmacological techniques (e.g. distraction) is insufficient to let the procedure take place in a comfortable manner.

Examples are: fracture reduction, repositioning of a luxation, dressing a large wound or a smaller wound in a very anxious child, draining an abscess, etc. Also so-called smaller procedures may fulfill the above description for (young and/or very anxious) children.

Based on its literature study, the working group has formulated the following recommendations for this group:

\section{Recommendation 45}

For very painful, stressful, and/or more complex procedures, a PSA must be chosen that generates deep sedation with a sufficient analgesic effect. The working group recommends that the following medication is chosen:

- $1^{\text {st }}$ choice: ketamine intravenously (i.v.) has been the subject of extensive research and has proven itself as an effective sedative. Its main disadvantage is the frequent occurrence of vomiting.

- $\quad 2^{\text {nd }}$ choice: propofol i.v. in combination with fentanyl i.v. has as a disadvantage that it is associated with a frequent occurrence of respiratory complications and a tendency for unwanted general anaesthesia to set in quickly.

\footnotetext{
* See also: 2.1. General recommendations for any procedure involving the administration of PSA.
} 
- $\quad 3^{\text {rd }}$ choice: midazolam in combination with fentanyl i.v. Like propofol, it has the disadvantage of the more frequent occurrence of respiratory complications.

If a decision is made to use sedatives and/or sedating analgesics, the preconditions in relation to patient selection, the choice of medicines, skills, competencies, and monitoring must be met, as well as the other preconditions set out in part I of these guidelines.

For biopsies, thorax drainage, and the dressing of burns, reference is made to the relevant chapters in this guideline (2.8., 2.9., 2.10).

\section{PAINFUL AND/OR LESS COMPLEX PROCEDURES}

Examples are the suturing of small wounds, injections, arterial or venous punctures, etc. See below 2.7. Periferous intravenous access procedures, punctures, and other brief painful and/or stressful procedures

\subsection{Periferous intravenous access procedures, punctures, and other brief painful and/or stressful procedures*}

Children in hospitals very frequently undergo relatively minor and brief procedures that may be painful and/or stressful to the children.

To reduce the fear, pain, and discomfort of these procedures, there are (1) nonpharmacological techniques, (2) local, and (3) systemic medicinal options. A combination of (1) and (2) will often suffice. It nevertheless frequently happens, particularly with small children, that these procedures cannot be properly managed without some form of restraint because the child resists a procedure that is experienced as painful and/or stressful. In many cases, children with a chronic disease undergo the same painful procedures very regularly, which means there is a risk of greater resistance and anticipatory anxiety. It can therefore be preferable to have access to a form of PSA for these small procedures.

Based on its literature study, the working group has formulated the following recommendations:

\section{Recommendation 46}

1. For small painful procedures, such as the insertion of a periferous drip, venapuncture, lumbar puncture, thorax puncture, joint punctures, the suturing of a wound, etc., the following recommendations apply:

a. Topical anaesthesia and one or more non-pharmacological techniques (childcentered approach, distraction, possibly hypnosis) must always be used in cases that do not involve an immediately life-threatening situation.

b. The time required for topical anaesthetics to take effect and for applying nonpharmacological techniques is negligible provided a sufficient anticipatory approach is used and it should therefore not be used as a pretext for not applying them.

c. With local sedation by means of an intradermal injection of lidocaine or Oberst's anaesthesia of fingers (and toes), the following precautionary measures must be observed:

\footnotetext{
* See also: 2.1. General recommendations for any procedure involving the administration of PSA.
} 
- use of a small, thin injection needle

- slow injection of lidocaine

- buffering lidocaine with bicarbonate

- heating up lidocaine to body temperature

2. For minor stressful but painless procedures (for example, a stomach tube, bladder catheterization, echography, or otoscopy) one or more non-pharmacological techniques (childcentered approach, distraction, possibly hypnosis) must always be used.

3. If forced immobilization and restraint are necessary or expected to be necessary for small painful and/or stressful procedures (see examples under 1 and 2), the first choice must be to use a light form of sedation or anxiolysis in addition to non-pharmacological techniques and (insofar as applicable) local anaesthesia. Midazolam and nitrous oxide can be used on those occasions.

a. In the event midazolam is not administered intravenously, it must preferably be used sublingually, in order to prevent irritation of the nose in the event of intranasal administration, ineffective sedation in the event of rectal administration, or an unpredictable plasma concentration in the event of oral administration.

If it is decided to use midazolam, it is therefore important to comply with the preconditions in terms of patient selection, skills, competencies, monitoring, and the other preconditions set out in part I of these guidelines. Although it cannot be readily substantiated with research data, a one-off administration of an initial dose of midazolam will usually only lead to anxiolysis/light sedation. Used in higher doses, in combination with other sedatives, midazolam will cause moderate to even deep sedation in some patients.

b. It is recommended to have access to nitrous oxide as a PSA technique for the many relatively minor yet painful procedures in children (e.g. blood tests, insertion of a drip, bladder catheterization, insertion of a stomach tube, suturing or dressing wounds, reducing fractures, and small surgical procedures). After all, this form of PSA is possibly more effective than the use of topical anaesthesia and/or anxiolysis with midazolam. The following additional benefits must also be taken into consideration: (1) the fact that no intravenous access is needed, (2) the less rigid conditions in terms of competencies, skills, and monitoring in comparison with deep sedation, (3) the rapid effect, and (4) the very brief recovery phase which enables a speedy discharge.

4. If it is (or can be) expected that light sedation or anxiolysis will not suffice for small painful and/or stressful procedures, it must be decided to use anaesthesia or a deep form of PSA. If it is decided to use deep PSA, the following recommendations apply:

a. Choice of medication:

1st choice: ketamine intravenously (i.v.); it has been the subject of extensive research and has proven itself as an effective sedative. Its main disadvantage is the frequent occurrence of vomiting.

2nd choice: propofol i.v. (possibly in combination with fentanyl i.v.); has as a disadvantage that it is associated with a frequent occurrence of respiratory complications and a tendency for unwanted general anaesthesia to set in quickly.

3rd choice: midazolam in combination with fentanyl i.v.; like propofol, it has the disadvantage of the more frequent occurrence of respiratory complications.

b. The preconditions set out in part I of this guideline in relation to patient selection, 
skills, competencies, and monitoring must be met.

\subsection{Thorax drainage*}

Inserting a thorax drain is a painful to very painful procedure that is performed urgently or semiurgently in patients with an underlying pulmonary and/or pleural problem. A proportion of these patients consequently also have respiratory problems. The latter has significant implications for the manner in which PSA is applied.

Based on its literature study, the working group has formulated the following recommendations:

\section{Recommendation 47}

1. Inserting a thorax drain in children must preferably be carried out under deep sedation and/or general anaesthesia.

2. If it is decided to use a PSA, then:

a. it must be supervised by an anaesthesiologist or an intensivist, to ensure that any deterioration in the respiratory condition can be adequately recognized and dealt with;

b. intravenous (i.v.) ketamine (possibly in combination with midazolam i.v.) is a suitable medicine. Other possibilities are propofol + local anaesthesia or remifentanil;

c. The preconditions in relation to patient selection, the choice of medicines, skills, competencies, and monitoring must be met, as well as the other preconditions set out in part I of these guidelines.

3. For older children and provided full, informed consent is obtained from the child, a decision can be made to use a combination of local anaesthesia and non-pharmacological techniques, possibly in combination with a form of anxiolysis (e.g. midazolam). This strategy is only acceptable provided the resulting situation does not then necessitate forced immobilization and restraint.

4. In connection with the potential expansion of a pneumothorax, nitrous oxide must not be used as PSA for inserting a thorax drain.

5. For a one-off pleural puncture, a combination of local anaesthesia and non-pharmacological techniques may suffice in cooperative patients.

\subsection{Caring for burns*}

Burns can cause (often severe) pain in three different ways. First, there is the acute pain immediately after the burns are caused. This pain is usually treated with (a combination of) (1) cooling, (2) local wound care and dressing, (3) enteral or intravenous first-line analgesics (paracetamol, NSAID), and (4) intravenous opiates. With extensive and $2^{\text {nd }}$ or $3^{\text {rd }}$ degree burns, it is preferable for the wounds to be initially cared for under the supervision of an anaesthesiologist, due to the amount of pain involved and/or the critical condition of the patient.

Second, burns and their treatment may give rise to continuous or basal pain. This pain can be stronger than expected as a result of hyperalgesia occurring in burnt areas. The treatment of

\footnotetext{
* See also: 2.1. General recommendations for any procedure involving the administration of PSA.
} 
this pain will usually consist of (a combination of) paracetamol, NSAID's, and opiates, but it sometimes requires help from pain specialists.

Third, there is procedural pain. This pain (and anxiety) arises in the context of the many and often prolonged periods during which the wounds are treated and the dressings are changed. As a result of hyperalgesia, even relatively minor wound care procedures can be extremely painful. Furthermore, since these procedures need to be repeated over a long period, this procedural pain (and often also anticipatory anxiety) is extremely stressful for the patient. Based on its literature study, the working group has formulated the following recommendations:

\section{Recommendation 48}

1. In children with burns, analgesia and minimizing stress and discomfort are essential in all phases of the treatment. An adequate PSA is therefore an essential component of the overall burns treatment.

2. PSA during the Acute Phase: In the event of extensive burns, the PSA during the wound treatment in the acute phase must be left to an anaesthesiologist or intensivist with competency in the deep sedation of seriously ill children, in view of the potentially critical condition of the patient.

3. PSA during the Non-Acute phase or for limited burns:

- Ketamine i.v. and fentanyl i.v. are effective forms of PSA in a child that still has intravenous access. Ketamine is preferable over fentanyl because it is more effective and creates less risk of respiratory complications.

- If the child cannot be given intravenous access, oral morphine, oral fentanyl, intranasal fentanyl, oral ketamine, and rectal ketamine can all be used as effective forms of PSA. It is impossible to indicate which of these options is best, based on the literature. Among the opiates, intranasal fentanyl seems to be the most controllable form.

4. Separation anxiety must be prevented or reduced during the treatment, in order to prevent post-traumatic stress syndrome. An effective PSA with adequate analgesia and an endeavor to ensure that the parent(s) or carer(s) is/are present during the procedure are therefore imperative measures.

\subsection{Liver and kidney biopsies*}

Based on its literature study, the working group has formulated the following recommendations:

\footnotetext{
* See also: 2.1. General recommendations for any procedure involving the administration of PSA.
} 


\section{Recommendation 49}

1. Liver and kidney biopsies in children must be performed under deep sedation and/or general anaesthesia.

2. If it is decided to use a PSA, intravenous ketamine (possibly in combination with midazolam i.v.) is a suitable medicine. Other possibilities are propofol + local anaesthesia or remifentanil.

3. For older children and provided full, informed consent is obtained from the child, a decision can be made to use a combination of local anaesthesia and non-pharmacological techniques, possibly in combination with a form of light sedation/anxiolysis (e.g. nitrous oxide or midazolam). This strategy is only acceptable provided the resulting situation does not then necessitate forced immobilization and restraint.

\subsection{Dental procedures}

In the Netherlands, PSA in paediatric dentistry does not form part of the basic curriculum for dentist training; it is only taught in postgraduate courses. For paediatric dentists working in the Netherlands (Dentist-Paediatric dentists, DPs), it forms part of their (post-doctoral) specialty training. In that way, it is easy to incorporate contact and communication as a basic skill, and subsequently PSA. PSA is increasingly administered in paediatric dentistry by DPs, who are either based in their own practice, or at a center for special dentistry or academic center.

Based on its literature study, the working group has formulated the following recommendations:

\section{Recommendation 50}

It is important that

- PSA in paediatric dentistry is fundamentally based on light (inhalation or oral) PSA.

- PSA is frequently used for minor invasive procedures. Nitrous oxide must be considered as the first choice, and midazolam as a substitute if nitrous oxide cannot be administered.

- PSA is considered as a component of influencing behavior.

- Clinicians must be experienced and trained.

- All preconditions in the domain of analgesia must be met.

- The entire process of PSA must take place under the authority of the clinician. 


\title{
CHAPTER 6
}

\section{Conclusions, general discussion and future perspectives}

\author{
Mapping out the path ahead
}

Teach thy tongue to say 'I don't know'and thou shalt progress

Maimonides (1135-1204) 
This thesis' journey has come to an end. However, looking over the landscape in front of us, we must conclude that this first stage was nothing more than a modest exploration of a vast wasteland that still needs to be exploited. Challenges as well as feelings of frustration may be in front of us. We certainly haven't reached the final goal yet, i.e. having an implemented solution to deal safely and effectively with procedure related stress, pain or non-cooperative behaviour in sick children. In fact, at this point we could even feel disappointed or confused, certainly on a higher scientific level, but still confused and without pragmatic answers. In case of an imminent desperation it is wise to sit down, to take a deep breath, to have some rest and - at first - to look back to the path that brought us to this point. Any sustained effort yields its inspiration.

\section{PART I: Looking back and analyzing the observed obstacles}

\section{CONCLUSION NUMBER 1: PSA practices in Dutch general paediatrics are unsafe}

In the last decade at least three serious accidents happened during PSA in children. At first sight the occurrence of these accidents seems surprising. PSA medicines traditionally used by Dutch general paediatricians, such as (combinations of) chloral hydrate, benzodiazepines, meperidine, lytic cocktails, are often considered safe sedatives that only cause light sedation. This general notion is expressed by the somewhat camouflaging terminology that is used in this respect. Terms like 'conscious sedation' or 'just a glow' ('roesje' in Dutch) suggest a safe practice based on innocent drugs. Given the low incidence rate of major complications, most professionals will also have no personal experience with PSA-related accidents. ${ }^{2-7}$ This may confirm the overall opinion that specific safety precautions are not indicated.

Nevertheless, in each of the three severe accidents that happened in The Netherlands during PSA in children, one of these 'innocent' drugs was involved. Recent research has unmasked the real characteristics of the traditional PSA practice. Coté was the first to report an association between the traditional PSA drugs and the occurrence of severe accidents. In his often cited critical incident analysis (published in 2000) of PSA related adverse events in children reported between 1969 and 1996 to the Food and Drug Administration, he found that adverse sedation events were frequently associated with the use of long-acting drugs, with drug overdoses and with drug interactions, particularly when 3 or more drugs were used. Adverse outcome was associated with all routes of drug administration and all classes of medication, even those (such as chloral hydrate) thought to have minimal effect on respiration. ${ }^{8}$ Hofmann et al. found in his multifactor analysis of PSA related safety that the use of chloral hydrate was an independent predictor of PSA related adverse events. ${ }^{3}$ Motas et al. clearly showed that in traditional sedatives the prediction of the sedation end point is absolutely unreliable. Chloral hydrate, lytic cocktails, benzodiazepine+opioid combinations, meperidine, or barbiturates, cause wide variations in depth of sedation, including 'excesses' to levels of deep sedation and even anaesthesia. ${ }^{9}$ Alternatively, an undesirably low level of sedation (baring the risk of procedural failure) may trigger the administration of supplementary doses or sedatives and may therefore cause 'oversedation'. It has been shown that an unexpectedly deep level of sedation occurring suddenly outside the safety net of appropriate and prolonged monitoring and adequate airway and rescue skills, is the most important risk factor for severe respiratory accidents, such as airway obstruction, hypoventilation, and apnoea, all leading to life threatening hypoxia. ${ }^{3,10}$ 
Cotés findings have had a tremendous impact on defining essential preconditions, skills and competence for PSA in children, mainly in terms of timely recognition and appropriate management of possible adverse events. Nowadays there exists a wide agreement that PSA related safety is determined by logistics, organization and professional skills rather than by specific pharmacologic characteristics. They form the essential components of all recently published safety guidelines on PSA in children (see chapter 1, part 2). In fact the Dutch 1998 CBO guideline on PSA recommended similar safety precautions. ${ }^{11}$

We showed that in Dutch general paediatrics the above mentioned traditional, long acting and moderately effective PSA drugs are still very commonly used. ${ }^{12}$ In addition we showed that the level of adherence to established safety guidelines on PSA is (very) low among general paediatricians. ${ }^{13}$ According to Coté's finding one decade ago, these two observations contain exactly the two main components of an unsafe PSA practice: the use of unpredictable and moderately effective sedatives within a context that lacks the safety precautions to detect and manage adverse events in time. This means that potentially unsafe PSA settings are widespread in Dutch general paediatrics. We strongly believe that the three severe accidents that happened in children during PSA for diagnostic imaging were not at all isolated events. Not changing the current PSA-setting in The Netherlands will certainly lead to similar accidents in the near future.

\section{CONCLUSION NUMBER 2: PSA practices in Dutch general paediatrics lack optimal effectiveness}

For two representative procedures in general paediatrics (i.e. Magnetic Resonance Imaging and Gastro-Intestinal Endoscopy) we have compared the current practice in The Netherlands with the results of systematic reviews on the most effective PSA technique. It turned out that loweffective PSA strategies and drugs are still commonly in use. As a consequence these (expensive and burdensome) medical procedures are often unsuccessful, uncomfortable or painful. The most important reasons for this ineffectiveness are the reduced predictability of the medicines used, the absence of good, coordinated care, and the limited competence of non-anaesthesiologists to accomplish a predictable depth of the sedation.

In addition, other scientific reports have shown that procedural pain management is still often inadequate, or that available analgesic and/or sedative techniques are not applied, leading to avoidable procedural stress and pain. ${ }^{14-17}$ Recent non-controlled observations by paediatric nurses in training showed that topical anaesthesia (e.g. Emla ${ }^{\circledR}$ ) is often not applied at all or only for a short and non-effective time preceding a painful puncture*. Similar observations suggest the frequent application of forced inmobilization ('restraint') during painful procedures in hospitalized children.

\section{CONCLUSION NUMBER 3: Ethical and legal considerations: 'primum non nocere' and children's right for optimal care}

Perhaps one of the more intriguing topics addressed in this thesis is an ethical and legal one. In children undergoing a medical procedure, we must weigh the need to perform that procedure against the child's wishes to be left untouched. Therefore we need to consider whether the benefits of a medical procedure justify causing significant pain and stress for that child. Most

\footnotetext{
* Heleen Stechele. Procedural comfort and the use of forced immobilization in sick children. Bachelor's thesis Paediatric Nursing. June 2011.
} 
importantly, if the knowledge and technology to perform sedation/analgesia for this procedure easily and painlessly exists, we cannot justify merely restraining a terrified child for a painful procedure because of the cost or extra efforts involved. This issue is highly important since it defines which sedation end-point should be considered as optimal for an individual child in a given procedure.

Current Dutch legislation holds that a young child ( $<12$ years of age) is not autonomous that is he/she is not at liberty to refuse needed treatment, as long as informed consent is obtained from parents or caregivers. In Chapter 4 we demonstrated that this reasoning is a too narrow-minded reading of the law. As (sedation) professionals, we need to recognize our obligation to make the experience of any procedure as tolerable as possible for each child. In giving sedation, we must strive to alter the experience of an uncomfortable or stressful situation, in which a given child may otherwise wilfully want to resist. It has been postulated that only if society ultimately considers physical restraint to accomplish a medical procedure a violation of a child's civil liberty - which is for example the case in Scotland - we will all become more committed to alternative solutions. ${ }^{18}$ We must be honest with parents and children about the nature of a given procedure and the possible options for altering perception of that procedure - be they emotional support, hypnosis, distraction techniques, anxiolytic/analgesic medications, or general anaesthesia. Perhaps most importantly, the consideration of these ethical principles requires providers to consider alternative plans for sedation of each child. Physical immobilization or restraint cannot be a surrogate excuse for withholding the most effective form of PSA. Fear of potentially unsafe deep sedation is important but must not be counterbalanced by unwanted emotional and psychological injury. Horrific accounts of painful procedures without effective PSA have caused posttraumatic stress disorder ${ }^{19-22}$. Furthermore, performing procedures in a struggling child might cause danger to both the patient and carers. ${ }^{23}$

The principle 'primum non nocere' and the basic right for optimal care also implies that the alternative, i.e. procedural sedation and/or analgesia, is in the patient's best interests and certainly not harmful in any way. This means that PSA needs to be optimally safe. Also potential toxicity of PSA drugs needs to be excluded. In that respect the recent concerns on the possible neurotoxicity of anaesthetics on the developing brain may be highly relevant. ${ }^{24}$ Although clinical importance remains to be substantiated, results to date do indicate that exposure of animals to general anaesthesia during active synaptogenesis is most detrimental. ${ }^{25}$ Given the recent trend to use potent anaesthetics like ketamine and propofol for PSA these observations may be relevant. However, during PSA patient is exposed only shortly to sedatives or anaesthetics, although repetitive exposure during a limited period of time may occur frequently, especially in chronically ill patients. Currently it is not known whether the experimental findings in animals can be simply extrapolated to human beings in general and to PSA in children in particular. Furthermore, the eventual (neuro)toxicity of non-anaesthetics such as barbiturates, benzodiazepines and chloral hydrate has never been subject of systematic research. Strikingly, chloral hydrate has been linked with genetoxicity and carcinogenicity and for those reasons it has been banned in France for use in children. ${ }^{26}$ Anyway, potential toxicity of potent PSA drugs must be counterbalanced to the potential biological and psychological consequences of ineffective sedation and repeatedly painful or distressing experiences during childhood. ${ }^{27}$ Additional research is needed to clarify this dilemma. 


\section{CONCLUSION NUMBER 4: Inadequate anaesthesia resources}

Due to a limited availability of anaesthesiologists, non-anaesthesiologists have developed their own PSA procedures. In chapter 1 we explained how this has caused a sinister paradox: untrained and incompetent professionals performing PSA out of sheer necessity or obstinacy versus trained and competent professionals (i.e. anaesthesiologists) not able or not willing to offer an appropriate PSA service; a dramatic condition resulting in non-transparency, ineffectiveness and, most outrageous at all, unsafe conditions for patient.

However, even optimal commitment by anaesthesiologists is not likely to be sufficient for solving the problem. As a result of the increase of invasive diagnostic or therapeutic procedures in sick or injured children, PSA is increasingly needed outside the operation theatre. These PSA services will also be required on short notice and during unpredictable hours (e.g., emergency department, intensive care unit). ${ }^{1}$ There are currently insufficient anaesthesiologists to provide procedural sedation and analgesia for all of these settings, and this situation is unlikely to change in the foreseeable future, not only in the Netherlands but in most European countries as well. ${ }^{28}$ Therefore non-anaesthesiologists will have to play a role in performing PSA in children. Fortunately, recent evidence indicates that in selected patients and procedures, PSA can be safely entrusted to trained non-anaesthesiologists, including PSA based on the use of the anaesthetics propofol and ketamine. ${ }^{29}$

\section{PART II: Identifying possible solutions}

\section{THE NEW GUIDELINE}

The new evidence-based guideline was intended to define the preconditions for safe and effective PSA in children (see Chapter 5). The process to compose this guideline took about 4 years of intense work and had two main results. At first the dialogue within the working group helped enormously to disentangle the sedation paradox. For the first time in my academic life I practically enjoyed the experience that Scientia vincere tenebras" ${ }^{\#}$. Secondly - of course - a new and scientifically correct guideline was produced, containing 50 practical recommendations how to make PSA optimally safe and effective in children. Based on similar experiences in other countries, the guideline authors strongly believe that the implementation of these recommendations will result in safe and effective PSA in children. The most import basic principles are summarized in Table 1.

Table 1. Basic Principles of the new Dutch evidence based guideline on PSA (CBO 2010)

1. PSA must be regarded as an independent medical treatment that must be entrusted to competent, specifically trained professionals only, in order to make it optimally safe and effective.

2. PSA is safe if the following preconditions are met: adequate patient selection, fasting status, monitoring of vital functions (in particular airway patency and breathing by use of capnography), monitored recovery until fully awake, rescue facilities and the professional

\footnotetext{
\# "By science overcoming the darkness" (Motto of the Free University Brussels - Belgium).
} 
competence to discover and manage in time any potentially dangerous adverse event.

3. The extent of the necessary preconditions and competencies are determined by (1) the intended level of sedation, (2) the sedation technique used, (3) the type of procedure to be carried out, and (4) the patient's individual characteristics and health.

4. The levels of sedation must be regarded as a continuous descending scale during which the patient may unexpectedly reach an inadvertent deeper level of sedation. The new Dutch guideline is the first to define on an evidence-based ground that the same safety preconditions and professional competences are needed for any level of sedation beyond the level of light sedation.

5. Sedation and analgesia are separate concepts

6. The most effective PSA technique should always be used in order to achieve an optimal procedural success. In addition, providing optimal relief for procedure related pain and anxiety is an ethical imperative.

7. The cornerstones of a comprehensive policy towards procedural success and comfort are:

a. The prevention of procedural pain and stress

b. An active policy to prevent forced immobilization and restraint

c. The optimal use of effective forms of local or topical anaesthesia

d. The systematic application of effective non-pharmacological techniques (preparation, distraction, hypnosis, etc.)

e. The application of the most adequate PSA technique, individually titrated and carried out by a trained professional

f. The ready availability of the so-called "rescue anaesthesia": this means that general anaesthesia is readily available in case a PSA technique turns out to be inadequate or if it can be anticipated that the available PSA techniques may be insufficient or unsafe in an individual patient.

\section{THE INTRODUCTION OF “NEW" PSA DRUGS}

Currently, drugs traditionally used in The Netherlands for PSA in children lack optimal effectiveness (See Chapter 2) due to the unpredictability of depth and duration of sedation. Ideally, each PSA should be directed to a specifically determined sedation level during a predictable time period. This makes the use of short acting "titratable" drugs advantageous over the use of long acting drugs. Short acting drugs can be used to overcome the pain and distress that varies according to the procedures and the patients themselves.

\section{Easily accessible deep sedation for 'major procedures': promising propofol}

For magnetic resonance imaging and gastrointestinal endoscopy procedures we have shown in two separate systematic reviews (see chapter 2) that a propofol-based PSA is superior to the more traditionally used PSA regimens. Propofol is an ultra-short acting anaesthetic and is widely used in anaesthesia. Its pharmacological characteristics (i.e. rapid onset and offset, optimal titratability to a desired level of sedation and brief recovery) make this drug a highly attractive drug for PSA. This is especially the case in the setting of a busy emergency room or diagnostic department where lengthy titrations of sedatives and personnel-intensive recoveries are unpractical. Consequently the use of propofol for PSA by non-anaesthesiologists is a growing practice. $^{30}$ 
Propofol is primarily a potent sedative and has only limited analgesic effects. When titrated up to a level of deep sedation or 'light anaesthesia' it may be used for both painless and painful procedures. ${ }^{31}$ However, in case of painful procedures, combined administration of fentanyl ${ }^{32}$ or ketamine ${ }^{33}$ should be considered. The high efficacy of propofol for PSA in children has been demonstrated in large series of patients in paediatric oncology ${ }^{32,34}$, critical care ${ }^{35}$, radiology ${ }^{36}$, gastroenterology ${ }^{37}$ and emergency medicine. ${ }^{38-40}$

The most important severe adverse effect of propofol is respiratory depression, which can arise suddenly and unexpectedly. ${ }^{41}$ Therefore propofol should only be used by competent professionals trained in its use and in the recognition and management of adverse events. Continuous capnography during PSA with propofol may detect airway compromise and respiratory depression before clinical observation or pulse oximetry. ${ }^{42}$ Other possible side effects are hypotension and pain during injection. At least in uncompromised patients hypotension is usually transient and in general clinically irrelevant. ${ }^{43}$ Adding lidocaïne to the ropofol solution can prevent pain during injection, which is more frequently seen in children compared to adults. ${ }^{44,45}$ Pre-treatment with a low-dose Ketamine may be associated with less infusion pain, more analgesia, less hypotension, a lowered dose of Propofol and consequently a lower risk of hypoventilation. ${ }^{44}$ An evidence-based clinical practice advisory for the administration of Propofol for emergency department PSA was recently published. ${ }^{46}$

Traditionally - and particularly as a consequence of the narrow respiratory safety margins the anaesthetic propofol has always been considered as "safe only in the hands of an anaesthesiologist." However recent evidence indicates that this 'privilege' cannot be justified any longer. ${ }^{47}$ Due to the limited anaesthesia resources ánd the high level of effectiveness of propofol, some professional groups of non-anaesthesiologists have been prominent in the application of propofol for PSA in children. Their research, including many ten thousands of well-reported propofol sedations, shows that in optimal safety and monitoring conditions and in well-trained hands the drug has a very high safety profile. ${ }^{35,39,43,48-50}$ Skills and competences, rather than professional title, are determinants for safe and effective use of Propofol. ${ }^{49}$ Propofol cán be safely administered by specifically trained non-anaesthesiologists for PSA in selected groups of patients and in specific procedures: major procedures (e.g. endoscopy, prolonged imaging, oncology procedures, emergency procedures, biopsies, major wound care,...) in low-risk paediatric patients (ASA I-II; normal airway(reflexes); absence of product-specific contraindications ${ }^{51}$ ) are eligible for propofol-based PSA performed by trained non-anaesthesiologists. In fact, the current situation in The Netherlands (i.e. moderately effective, long-acting drugs administrated by the untrained in a setting of non-adherence to guidelines) is likely to cause substantially more severe accidents than a transparent PSA setting where highly active drugs like propofol are used by competent professionals, including well-trained non-anaesthesiologists. ${ }^{29}$, 48 Unfortunately and despite the scientific evidence, a collective of several European Societies of Anaesthesia has recently advised strongly against the use of propofol by non-anaesthesiologists. ${ }^{52}$ Hopefully, this will not result in resuming the old positional conflict between anaesthesiologists and nonanaesthesiologists regarding PSA and consequently in denying access for sick children to safe and effective PSA.

Finally, it must be noted that the pharmaceutical manufacturer of propofol recommends not using the drug for sedation in children under the age of 16 years because of possible toxicity. Metabolic acidosis, rhabdomyolisis, hyperpotassemia and fatal cardiac failure have been observed in children receiving high doses of propofol perfusions (>5 mg/kg/hr). Lactic acidosis and torsades de pointes have been reported in lower doses administered for at least $24 \mathrm{hrs} .{ }^{53} \mathrm{How}-$ ever, similar toxic effects have never been observed during short-term administration for PSA. 
Not to forget the so-called 'minor procedures': the need for nitrous oxide sedation

Needle-related distress has a substantial impact on children and their parents, and may lead to management problems and treatment refusal, especially in children with chronic diseases. ${ }^{\mathbf{5 4}}$ Psychological and pharmacological interventions may reduce distress substantially. For the socalled 'minor procedures' (a terminology clearly based on the performer's perspective...), like intravenous access, blood sampling, suturing, wound care, bladder catheterisation, lumbar puncture... the new guideline recommends the use of nitrous oxide (N2O) to relieve procedure related pain/distress and to improve cooperative behaviour. Nitrous oxide has enormous potentials for use in general paediatrics and emergency medicine, in particular to make so called 'minor procedures' comfortable. Effectiveness is maximal when it is combined with child-friendly circumstances, distraction techniques and optimal topical anaesthesia. ${ }^{55}$ Safety and effectiveness have been thoroughly demonstrated in the last decade. ${ }^{56-65}$ Only in very specific categories of vulnerable patients the use of $\mathrm{N} 2 \mathrm{O}$ is contraindicated (e.g. patients with abnormal vitamin B12 and B12-related metabolic disorders). ${ }^{66}$

Strikingly - and contrary to the USA and most other European countries - the use of nitrous oxide has become quite controversial in the Netherlands, in terms of the potential toxic effects for the staff administering it (namely: toxicity to an unborn child and effects on fertility). The (theoretically-based) recognition of potential problems of occupational exposure of $\mathrm{N}_{2} \mathrm{O}$ has led internationally to the introduction of occupational exposure limits (OEL), expressed as 8-hours time-weighed averages (TWA in parts per million (ppm)). Strikingly there is no clear consensus on which is an appropriate OEL, resulting in TWA limits ranging from 25 ppm (e.g New Zealand, Australia, USA) over 50 ppm (Norway, Denmark, Spain) and 80 ppm (The Netherlands) to 100 ppm (Finland, Sweden, United Kingdom, Switzerland). Canada has three different OELs (25 ppm (Ontario), $50 \mathrm{ppm}$ (Quebec) and $100 \mathrm{ppm}$ (Alberta), whereas France has no specifically defined OELs for $\mathrm{N}_{2} \mathrm{O}$. None of these limits has any scientific basis. At generally accepted occupational exposure limits, there is no conclusive evidence for reproductive, genetic, haematologic or neurologic toxicity from nitrous oxide exposure. ${ }^{67}$ Furthermore, the often cited studies by Rowland et al. suggesting health risks associated with higher OEL's (TWA $>1000 \mathrm{ppm}$ ) lack sufficient power and the methodological correctness to prove such an assumption. ${ }^{68,69}$ The very strict rules regarding ambient pollution make it currently practically impossible for Dutch health care to apply the inhalation techniques that are commonly in use for Nitrous oxide administration in the rest of the Western medical world. Of course, only competent professionals trained in its adequate and safe use, including minimizing ambient pollution, should use nitrous oxide. However, in order to meet the current occupational health instructions in The Netherlands, highly expensive and even child-unfriendly measures would be needed (e.g. intensively ventilated spaces, high-flow 'Hoover-like' source suctioning around the facial mask, special exhaust piping outdoors...). The current legislation feels like throwing the baby out with the bathwater: scarcely funded safety precautions shut the door to a highly effective PSA technique. Personally, I strongly believe that every effort must be made to make nitrous oxide mixtures available in The Netherlands for PSA in children. For that reason, special initiatives need to be developed in order to ensure that the legislation currently in force on the administration of nitrous oxide no longer hinders the widespread distribution of the technique. At the same time training and techniques of safe administration should become available. A pilot project at the Procedural Sedation Unit of the Maastricht University Hospital, using a mobile $\mathrm{N}_{2} \mathrm{O}$ administrationscavenging-destruction unit for light sedation in children, is currently generating promising data, both on the effectiveness in children as well as on the reduction of ambient air pollution. 


\section{PART III: Looking forward: mapping out the route ahead}

It is a good and humble tradition to finish a medical research report with the words 'more research needs to be done...'. The latter is - however - not really the case in this respect. Currently we have sufficient scientific evidence to our disposal to know which drugs, techniques and settings result in highly safe and effective PSA in children. That does not mean that the search for knowledge has come to an end. For example, promising new data keep on appearing, e.g. PSA with dexmedetomidine, inhalation anaesthetics and the use of mucosal atomization devices $\left(\mathrm{MAD}^{\circledR}\right)$ for the nasal administration of benzodiazepines and ketamine. Nevertheless, not 'more research' but rather 'how to implement the current knowledge' (as summarized in the new Dutch guideline) should be the first (and much more challenging) target. It is not likely to find obstinate opponents disputing the statement that there is an urgent need for 'Change' in the PSA field. However, it is likewise unlikely that just shouting 'yes we can' will result in a definite change.

The new guideline aims to profoundly change the practice of PSA in children in the Netherlands, in order to make it optimally safe and effective. In modern medicine, guidelines are increasingly being designed by and spread among health care professionals in an attempt to improve the professional performance, health care process, outcomes and costs. Guidelines can be defined as systematically formulated documents that assist practitioners to make clinical decisions informed by the best available evidence. ${ }^{70}$ However, many studies have shown that the mere designing, publishing and disseminating of guidelines does not necessarily imply the intended positive change in daily practice. ${ }^{71-74}$ Passive dissemination of guidelines among professionals has been shown to be ineffective. ${ }^{70}$ Implementation experts agree that successful implementation of a guideline requires extensive planning, education, skill training and behavioural methods (e.g. feedback, continuous evaluation of adherence behaviour) but even so marketing techniques, economic factors and social pressure. ${ }^{74,75}$

With that in mind, PSA must at first obtain the status of a formal and transparent medical treatment with clearly defined skill sets and competencies. However, because of (the aftereffects of) the long-lasting sedation paradox, the current twilight-zone status of most PSA practices and the non-existence of a well-defined training in PSA, paediatric professionals cannot be expected to implement the new recommendations in their personal practice at the time the new guideline is ratified.

Therefore a carefully considered implementation plan is crucial.

\section{From Guideline to implementation: ideas on how to implement the guideline}

\section{A national support group}

This group, consisting of PSA experts and representatives of the most important scientific associations, will coordinate the implementation of the guidelines. Ideally, this working group, supported by implementation experts, roll out pilot-projects in selected settings and hospitals. In addition this group may function as a help-desk as well as a visitation committee.

\section{A local PSA quality-monitoring group}

This group, functioning as an official hospital committee, will be responsible for the local implementation of the recommendations in the guidelines, the issue of PSA competence certificates, quality control, drawing up local protocols and local training. In this group all relevant specialties involved in providing PSA and/or in need for PSA must be represented. Anaesthesiologist should 
take a leading role in the local coordination of PSA services (see further on: the issue of credentialing).

\section{Training}

With the exception of dental sedation, there are currently no national training programs or qualifications for PSA in children. It is difficult to design a universal training schedule for the many different types of sedation, some of which will not be relevant for all specialists.

A seemingly straightforward skill that all sedationists should have is airway management and resuscitation. Besides, a training program must teach general PSA competencies in the following domains: screening and pre-PSA care, administration of PSA, care after PSA, policy, education, research, and legal aspects. Although training, competences and required experience will certainly vary according to specific situations and settings in which PSA will be applied, we believe that overall two different levels of training and certification are needed:

\section{LEVEL I: Training to become a 'specialist in PSA practice'}

Although the need for PSA during interventions has increased enormously over the last few decades, there is a lack of specific PSA training within the relevant specialties and nurse training courses. Due to the persistent shortage of anaesthesiologists in the labour market, whilst the clinical workload has grown, anaesthesiologists are not likely to be able to fulfil the burgeoning demand for PSA. On the other hand the new guidelines recommend that titratable deep sedation be made available to patients, including the use of highly active drugs like propofol. This requires the commitment of professionals who are trained to perform safely and effectively PSA intended to moderate and deep sedation levels. Well-trained non-anaesthesiologists may be entrusted with PSA without creating additional risks. A training system needs therefore to be developed to train practice-based PSA specialists. The authority to work as a specialist in PSA practice can be achieved through different routes. This training can be built in into medical specialty follow-on training, but it can also be designed as a separate course. It is therefore important to list the necessary levels of knowledge and skills that must be achieved before trainees can call themselves 'specialists in PSA practice for children'. In addition, it is advisable to set up a master's degree for a physician assistant in anaesthesiology, with competencies in the fields of sedation/analgesia, pre-PSA screening, and in the domain of pain control. This professional would be capable of providing screening and performing safe and effective moderate to deep PSA as required by imaging procedures, gastrointestinal endoscopy procedures, oncology procedures, very painful emergency procedures or other procedures of similar nature.

\section{LEVEL II: Training to become competent in light sedation/anxiolysis}

It is advisable to establish training in anxiolysis/light sedation for professionals involved in the medical care for children who need to undergo short painful and/or mildly stressful procedures (e.g. simple wound care, venous access, lumbar puncture, blood sampling, bladder catheterization...). Such training is geared to the use of (1) light types of PSA (for instance nitrous oxide), (2) non-pharmacological techniques (child-oriented approach, distraction techniques and hypnosis), and (3) topical anaesthesiology techniques. The most important goal is to optimize comfort during the procedure and reduce the necessity of coercion or restraint during the procedure. The target audience consists of paediatricians, paediatric nurses and emergency care professionals. 
Training methods: the potentials of human patient simulation (HPS)

Access to live patients is a limiting factor for sufficient training and the development of life-like manikins is a potential solution. European resuscitation courses are widespread but do not aim to teach the monitoring and proactive airway skills that sedationists need. HPS should be a common component of specialty-specific sedation training courses as well as training in light sedation/anxiolysis. HPS has the enormous advantage of offering a controlled environment in which to introduce learners to clinical situations, including those that are relatively infrequent. Modern HPS technology allows creating simulated settings that closely resemble actual care. A first attempt to show the potentials of HPS for the training of paediatric sedation providers was published by Shavit at. In a small cohort of paediatric sedation providers they showed that HPSbased training resulted in an improved PSA safety performance. ${ }^{76,77}$ In that perspective, existing Dutch training centres with substantial HPS training expertise (e.g. Stichting Spoedeisende Hulp bij Kinderen (SSHK)) could play a major role in the setting up of dedicated trainings for one or both intended levels.

\section{The importance of credentialing competent non-anaesthesiologists}

The scientifically-based trend to entrust the PSA practice (including deep sedation and even 'light propofol anaesthesia') to specifically trained and dedicated professionals, (including nonanaesthesiologists) may interfere with traditional views and (and even legally assigned) anaesthesiology privileges. This process of credentialing that is only in the very first stages of development in only a few European countries, will certainly need time and debate. Much inspiration for the near future can be learned from the USA experience in the two past decades. Four strategies that could move us towards credentialing have been clearly identified by Krauss and Green. Based on their American experience they defined 4 possible courses how anaesthesiologists could respond to PSA being practiced by other specialties. (Table 3) We favour the option of creating safe and effective services controlled by the institution who take their direction from national and professional guidelines (option 4). ${ }^{1}$ Such a system should bring development of efficient training that may evolve into national training schedules.

Table 3: Krauss \& Green: Four possible courses of action ${ }^{1}$

1. Anaesthesiologists should regulate all procedural sedation and analgesia and maintain full authority over the process.

2. Contrary to the first course of action, instead adopt a laissez faire approach. Provide each specialty the flexibility to define and enforce its procedural sedation and analgesia practice within the confines of major national or local mandates, but otherwise without anaesthesiologists oversight.

3. A third approach would be to adopt the USA model in which hospitals delegate authority for sedation leadership to either an individual or a multidisciplinary hospital-wide sedation committee. This entity would enact and enforce locally customized sedation policies.

4. A final approach, which is less regulatory and more proactive, is to create hospital-wide sedation committees to teach and be a resource. They would not erect undue barriers unless there was compelling evidence of not meeting national standards of care, but rather oversee an open and non-threatening dialog on optimal procedural sedation and analgesia practice so that various specialists can learn from each other.

Anaesthesiologists should 'embrace' the unique opportunity to show leadership in shaping the PSA practice according to their own well-established quality norms and in training sedation practitioners. In a recent editorial, commenting on the recent appeal of certain European Societies of Anaesthesia to forbid the use of propofol for PSA by non-anaesthesiologists (see page $288^{52}$ ), Werner et al. recommend their European anaesthesiology colleagues to use their influ- 
ence and expertise to create the right conditions for skilled sedation as this "can only enhance the quality and safety of sedation practice throughout Europe". They conclude by stating "it would be unfortunate if fundamentalism and populism were to weaken our position as a profession". ${ }^{78}$

\section{Financial issues}

Changing the actual PSA practice will certainly cost money. A crucial question is how eager and willing society, in general, and health care authorities and insurance companies, in particular, will be to invest in an improvement of PSA related safety and effectiveness. Given the current socio-economical situation, there are some reasons to be somewhat pessimistic. It will be essential to demonstrate that implementing the new guideline is justifiable from a cost-benefit point of view. Probably, the 'burden' of necessary investments to achieve more effective PSA services (e.g. training, new professionals, accessibility of propofol and nitrous oxide, appropriate monitoring and recovery, timely availability on a $24 \mathrm{~h}$ basis) can be quite easily calculated and will create immediately strong barriers for change. Calculating the economical aspects of the benefits will be much harder. In calculating these benefits one should at first calculate the costs of the current PSA situation and its consequences on unsafety and ineffectiveness. No one currently knows the direct and indirect economical costs of procedural failure due to ineffective sedation in children (e.g. imaging or endoscopy procedures). Furthermore there are only a few studies on paediatric PSA that have incorporated economical costs as an outcome measure. Kain et al. compared propofol-based procedural sedation with intravenous thiopental/pentobarbital sedation for children undergoing magnetic resonance imaging. A preliminary cost analysis was applied to the clinical data obtained and to a theoretical model of a paediatric MRI centre. Cost analysis of the propofol-based services revealed added drug costs $(\$ 1600.76$ per year for the propofol group) but significant savings of post-sedation care unit (PACU) nursing time ( $\$ 5086.67$ per year). ${ }^{79}$ Ekbom et al published a randomized controlled study in children with difficulties in establishing venous access or anxious children in need for an IV access. The patients were randomised to conventional treatment (i.e. cutaneous application of EMLA) or nitrous oxide treatment. They concluded "the pre-treatment with nitrous oxide is a time effective and safe method to reduce pain, facilitate venous cannulation, and thereby reduce the number of costly cancellations of planned procedures". ${ }^{56}$

A major issue regarding this debate is the fact that the moderately effective drugs that are currently widely in use in paediatric PSA are associated with a real risk of complications. Their use must therefore always be combined with extensive precautionary measures and the presence of professionals who can deal with any complications. ${ }^{41} \mathrm{~A}$ recent systematic review (see chapter 4) demonstrated that the same preconditions, level of monitoring, and emergency provisions apply for moderate (e.g. caused by chloral hydrate) and deep (e.g. caused by propofol) sedation. ${ }^{80}$ Optimizing these preconditions requires significant investment in training and infrastructure. Although such interventions will increase patient safety, they can only be costefficient provided they actually result in optimal effectiveness. ${ }^{79}$ However, even in optimal safety conditions, most standard PSA drugs will never result in an optimal effectiveness. Only dedicated PSA services making use of titratable short-acting drugs within a strict context of safety and competence will be able to combine optimal safety and effectiveness.

A profound cost-benefit analysis by health scientists and economists could throw more light on this matter. So, 'more research' could help. However, as clinicians we should do everything to guarantee optimal patient care. Any critical cost-analysis that questions evidence-based optimal standards for patient safety, procedural success and patient comfort should be regarded as 
potentially unethical. We should only be satisfied with a strategy that guarantees optimal procedural success, while maintaining maximal safety and minimal pain or anxiety. Any approach less than this threatens fundamental patient's rights and may harm the children we care for. 


\section{References}

1. Krauss B, Green SM. Training and credentialing in procedural sedation and analgesia in children: lessons from the United States model. Paediatric anaesthesia. 2008;18(1):30-35.

2. Cravero JP, Blike GT, Beach M, Gallagher SM, Hertzog JH, Havidich JE, et al. Incidence and nature of adverse events during pediatric sedation/anesthesia for procedures outside the operating room: report from the Pediatric Sedation Research Consortium. Pediatrics. 2006;118(3):1087-1096.

3. Hoffman GM, Nowakowski R, Troshynski TJ, Berens RJ, Weisman SJ. Risk reduction in pediatric procedural sedation by application of an American Academy of Pediatrics/American Society of Anesthesiologists process model. Pediatrics. 2002;109(2):236-243.

4. Malviya S, Voepel-Lewis T, Eldevik OP, Rockwell DT, Wong JH, Tait AR. Sedation and general anaesthesia in children undergoing MRI and CT: adverse events and outcomes. British journal of anaesthesia. 2000;84(6):743-748.

5. Malviya S, Voepel-Lewis T, Tait AR. Adverse events and risk factors associated with the sedation of children by nonanesthesiologists. Anesthesia and analgesia. 1997;85(6):1207-1213.

6. Maxwell LG, Tobias JD, Cravero JP, Malviya S. Adverse effects of sedatives in children. Expert opinion on drug safety. 2003;2(2):167-194.

7. Sanborn PA, Michna E, Zurakowski D, Burrows PE, Fontaine PJ, Connor L, et al. Adverse cardiovascular and respiratory events during sedation of pediatric patients for imaging examinations. Radiology. 2005;237(1):288-294.

8. Cote CJ, Karl HW, Notterman DA, Weinberg JA, McCloskey C. Adverse sedation events in pediatrics: analysis of medications used for sedation. Pediatrics. 2000;106(4):633-644.

9. Motas D, McDermott NB, VanSickle T, Friesen RH. Depth of consciousness and deep sedation attained in children as administered by nonanaesthesiologists in a children's hospital. Paediatric anaesthesia. 2004;14(3):256-260.

10. Cote CJ, Notterman DA, Karl HW, Weinberg JA, McCloskey C. Adverse sedation events in pediatrics: a critical incident analysis of contributing factors. Pediatrics. 2000;105(4 Pt 1):805-814.

11. Knape J. Sedation and/or analgesia by non-anesthesiologists [Sedatie en/of Analgesie door nietanesthesiologen]. www.cbo.nl. www.cbo.nl. Published 1998.

12. Leroy PL, Verbeke JIML, F.H.M. N, Blokland-Loggers HE. Succesvolle en veilige sedatie voor MRI onderzoek bij kinderen: de dagelijkse praktijk in Nederland getoetst aan de resultaten van een systematic review van de literatuur. Tijdschrift voor Kindergeneeskunde. 2011;79(2):42-54.

13. Leroy PL, Nieman FH, Blokland-Loggers HE, Schipper DM, Zimmermann LJ, Knape JT. Adherence to safety guidelines on paediatric procedural sedation: the results of a nationwide survey under general paediatricians in The Netherlands. Archives of disease in childhood. 2010.

14. Alexander J, Manno M. Underuse of analgesia in very young pediatric patients with isolated painful injuries. Annals of emergency medicine. 2003;41(5):617-622.

15. MacLean S, Obispo J, Young KD. The gap between pediatric emergency department procedural pain management treatments available and actual practice. Pediatric emergency care. 2007;23(2):87-93.

16. Loryman B, Davies F, Chavada G, Coats T. Consigning "brutacaine" to history: a survey of pharmacological techniques to facilitate painful procedures in children in emergency departments in the UK. Emerg Med J. 2006;23(11):838-840.

17. Bhargava R, Young KD. Procedural pain management patterns in academic pediatric emergency departments. Acad Emerg Med. 2007;14(5):479-482.

18. Cravero JP, Blike GT. Pride, Prejudice, and Pediatric Sedation:A Multispecialty Evaluation of the State of the Art. In: Report from a Dartmouth Summit on Pediatric Sedation: National Patient Safety Foundation; 2001.

19. Daviss WB, Mooney D, Racusin R, Ford JD, Fleischer A, McHugo GJ. Predicting posttraumatic stress after hospitalization for pediatric injury. Journal of the American Academy of Child and Adolescent Psychiatry. 2000;39(5):576-583. 
20. Daviss WB, Racusin R, Fleischer A, Mooney D, Ford JD, McHugo GJ. Acute stress disorder symptomatology during hospitalization for pediatric injury. Journal of the American Academy of Child and Adolescent Psychiatry. 2000;39(5):569-575.

21. Hain RD, Campbell C. Invasive procedures carried out in conscious children: contrast between North American and European paediatric oncology centres. Archives of disease in childhood. 2001;85(1):1215.

22. Rennick JE, Johnston CC, Dougherty G, Platt R, Ritchie JA. Children's psychological responses after critical illness and exposure to invasive technology. J Dev Behav Pediatr. 2002;23(3):133-144.

23. Rechtbank's-Hertogenbosch 26 januari 2011, Medisch Contact 2011; 808-810.

24. Davidson AJ. Anesthesia and neurotoxicity to the developing brain: the clinical relevance. Paediatr Anaesth 2011 Jul;21(7):716-21 doi: 101111/j1460-9592201003506x Epub 2011 Apr 6.21(7):716-721.

25. Jevtovic-Todorovic $V$. Anesthesia and the developing brain: are we getting closer to understanding the truth? Curr Opin Anaesthesiol 2011 Jun 8 [Epub ahead of print].

26. Gauillard J, Cheref S, Vacherontrystram MN, Martin JC. [Chloral hydrate: a hypnotic best forgotten?]. L'Encephale. 2002;28(3 Pt 1):200-204.

27. Green SM, Cote CJ. Ketamine and neurotoxicity: clinical perspectives and implications for emergency medicine. Annals of emergency medicine. 2009;54(2):181-190.

28. Egger Halbeis CB, Cvachovec K, Scherpereel P, Mellin-Olsen J, Drobnik L, Sondore A. Anaesthesia workforce in Europe. European journal of anaesthesiology. 2007;24(12):991-1007.

29. Couloures KG, Beach M, Cravero JP, Monroe KK, Hertzog JH. Impact of provider specialty on pediatric procedural sedation complication rates. Pediatrics 2011.127(5):e1154-1160.

30. Cravero JP, Blike GT. Pediatric sedation. Current opinion in anaesthesiology. 2004;17(3):247-251.

31. Mace SE, Barata IA, Cravero JP, Dalsey WC, Godwin SA, Kennedy RM, et al. Clinical policy: evidencebased approach to pharmacologic agents used in pediatric sedation and analgesia in the emergency department. Annals of emergency medicine. 2004;44(4):342-377.

32. Nagel K, Willan AR, Lappan J, Korz L, Buckley N, Barr RD. Pediatric oncology sedation trial (POST): A double-blind randomized study. Pediatric blood \& cancer. 2008;51(5):634-638.

33. Akin A, Esmaoglu A, Tosun Z, Gulcu N, Aydogan H, Boyaci A. Comparison of propofol with propofolketamine combination in pediatric patients undergoing auditory brainstem response testing. International journal of pediatric otorhinolaryngology. 2005;69(11):1541-1545.

34. Hertzog JH, Dalton HJ, Anderson BD, Shad AT, Gootenberg JE, Hauser GJ. Prospective evaluation of propofol anesthesia in the pediatric intensive care unit for elective oncology procedures in ambulatory and hospitalized children. Pediatrics. 2000;106(4):742-747.

35. Hertzog JH, Campbell JK, Dalton HJ, Hauser GJ. Propofol anesthesia for invasive procedures in ambulatory and hospitalized children: experience in the pediatric intensive care unit. Pediatrics. 1999;103(3):E30.

36. Pershad J, Gilmore B. Successful implementation of a radiology sedation service staffed exclusively by pediatric emergency physicians. Pediatrics. 2006;117(3):e413-422.

37. Barbi E, Gerarduzzi T, Marchetti F, Neri E, Verucci E, Bruno I, et al. Deep sedation with propofol by nonanesthesiologists: a prospective pediatric experience. Archives of pediatrics \& adolescent medicine. 2003;157(11):1097-1103.

38. Bassett KE, Anderson JL, Pribble CG, Guenther E. Propofol for procedural sedation in children in the emergency department. Annals of emergency medicine. 2003;42(6):773-782.

39. Guenther E, Pribble CG, Junkins EP, Jr., Kadish HA, Bassett KE, Nelson DS. Propofol sedation by emergency physicians for elective pediatric outpatient procedures. Annals of emergency medicine. 2003;42(6):783-791.

40. Pershad J, Godambe SA. Propofol for procedural sedation in the pediatric emergency department. The Journal of emergency medicine. 2004;27(1):11-14.

41. Krauss B, Green SM. Procedural sedation and analgesia in children. Lancet. 2006;367(9512):766-780.

42. Anderson JL, Junkins E, Pribble C, Guenther E. Capnography and depth of sedation during propofol sedation in children. Annals of emergency medicine. 2007;49(1):9-13. 
43. Green SM, Krauss B. Propofol in emergency medicine: pushing the sedation frontier. Annals of emergency medicine. 2003;42(6):792-797.

44. Badina L, Norbedo S, Barbi E. Procedural sedation and analgesia in children. Lancet. 2006;367(9526):1900-1901; author reply 1901.

45. Rochette A, Hocquet AF, Dadure C, Boufroukh D, Raux O, Lubrano JF, et al. Avoiding propofol injection pain in children: a prospective, randomized, double-blinded, placebo-controlled study. British journal of anaesthesia. 2008;101(3):390-394.

46. Miner JR, Burton JH. Clinical practice advisory: Emergency department procedural sedation with propofol. Annals of emergency medicine. 2007;50(2):182-187, 187 e181.

47. Cote CJ. Round and round we go: sedation -- what is it, who does it, and have we made things safer for children? Paediatric anaesthesia. 2008;18(1):3-8.

48. Cravero JP, Beach ML, Blike GT, Gallagher SM, Hertzog JH. The incidence and nature of adverse events during pediatric sedation/anesthesia with propofol for procedures outside the operating room: a report from the Pediatric Sedation Research Consortium. Anesthesia and analgesia. 2009;108(3):795-804.

49. Green SM, Krauss B. Barriers to propofol use in emergency medicine. Annals of emergency medicine. 2008;52(4):392-398.

50. Hertzog JH, Havidich JE. Non-anesthesiologist-provided pediatric procedural sedation: an update. Current opinion in anaesthesiology. 2007;20(4):365-372.

51. Kiringoda R, Thurm AE, Hirschtritt ME, Koziol D, Wesley R, Swedo SE, et al. Risks of propofol sedation/anesthesia for imaging studies in pediatric research: eight years of experience in a clinical research center. Archives of pediatrics \& adolescent medicine.164(6):554-560.

52. Perel A. Non-anaesthesiologists should not be allowed to administer propofol for procedural sedation: a position paper of 21 european national societies of anaesthesia. European Journal of Anaesthesiology 2011 Jun 23 [Epub ahead of print]. 2011.

53. Nederlands Kenniscentrum Farmacotherapie bij Kinderen www.kinderformularium.nl.

54. Ayers S, Muller I, Mahoney L, Seddon P. Understanding needle-related distress in children with cystic fibrosis. British journal of health psychology.16(Pt 2):329-343.

55. Brown SC, Hart G, Chastain DP, Schneeweiss S, McGrath PA. Reducing distress for children during invasive procedures: randomized clinical trial of effectiveness of the PediSedate. Paediatric anaesthesia. 2009;19(8):725-731.

56. Ekbom K, Jakobsson J, Marcus C. Nitrous oxide inhalation is a safe and effective way to facilitate procedures in paediatric outpatient departments. Archives of disease in childhood. 2005;90(10):1073-1076.

57. Ekbom K, Kalman S, Jakobsson J, Marcus C. Efficient Intravenous Access Without Distress: A Doubleblind Randomized Study of Midazolam and Nitrous Oxide in Children and Adolescents. Arch Pediatr Adolesc Med 2011 May 2 [Epub ahead of print]

58. Ekbom K, Lindman N, Marcus C, Anderson RE, Jakobsson JG. Health aspects among personnel working with nitrous oxide for procedural pain management in children. Acta anaesthesiologica Scandinavica. 2008;52(4):573-574.

59. Reinoso-Barbero F, Pascual-Pascual SI, de Lucas R, Garcia S, Billoet C, Dequenne V, et al. Equimolar nitrous oxide/oxygen versus placebo for procedural pain in children: a randomized trial. Pediatrics.127(6):e1464-1470.

60. Babl FE, Oakley E, Seaman C, Barnett P, Sharwood LN. High-concentration nitrous oxide for procedural sedation in children: adverse events and depth of sedation. Pediatrics. 2008;121(3):e528-532.

61. Babl FE, Puspitadewi A, Barnett P, Oakley E, Spicer M. Preprocedural fasting state and adverse events in children receiving nitrous oxide for procedural sedation and analgesia. Pediatric emergency care. 2005;21(11):736-743.

62. Gall O, Annequin D, Benoit G, Glabeke E, Vrancea F, Murat I. Adverse events of premixed nitrous oxide and oxygen for procedural sedation in children. Lancet. 2001;358(9292):1514-1515.

63. Kanagasundaram SA, Lane LJ, Cavalletto BP, Keneally JP, Cooper MG. Efficacy and safety of nitrous oxide in alleviating pain and anxiety during painful procedures. Archives of disease in childhood. 2001;84(6):492-495. 
64. Burnweit C, Diana-Zerpa JA, Nahmad MH, Lankau CA, Weinberger M, Malvezzi L, et al. Nitrous oxide analgesia for minor pediatric surgical procedures: an effective alternative to conscious sedation? Journal of pediatric surgery. 2004;39(3):495-499; discussion 495-499.

65. Paut O, Calmejane C, Delorme J, Lacroix F, Camboulives J. EMLA versus nitrous oxide for venous cannulation in children. Anesthesia and analgesia. 2001;93(3):590-593.

66. Baum VC. When nitrous oxide is no laughing matter: nitrous oxide and pediatric anesthesia. Paediatric anaesthesia. 2007;17(9):824-830.

67. Sanders RD, Weimann J, Maze M. Biologic effects of nitrous oxide: a mechanistic and toxicologic review. Anesthesiology. 2008;109(4):707-722.

68. Rowland AS, Baird DD, Shore DL, Weinberg CR, Savitz DA, Wilcox AJ. Nitrous oxide and spontaneous abortion in female dental assistants. American journal of epidemiology. 1995;141(6):531-538.

69. Rowland AS, Baird DD, Weinberg CR, Shore DL, Shy CM, Wilcox AJ. Reduced fertility among women employed as dental assistants exposed to high levels of nitrous oxide. The New England journal of medicine. 1992;327(14):993-997.

70. Prior M, Guerin M, Grimmer-Somers K. The effectiveness of clinical guideline implementation strategies-a synthesis of systematic review findings. Journal of evaluation in clinical practice. 2008;14(5):888-897.

71. Sheldon TA, Cullum N, Dawson D, Lankshear A, Lowson K, Watt I, et al. What's the evidence that NICE guidance has been implemented? Results from a national evaluation using time series analysis, audit of patients' notes, and interviews. BMJ (Clinical research ed. 2004;329(7473):999.

72. Grimshaw J, Eccles M, Thomas R, MacLennan G, Ramsay C, Fraser C, et al. Toward evidence-based quality improvement. Evidence (and its limitations) of the effectiveness of guideline dissemination and implementation strategies 1966-1998. Journal of general internal medicine. 2006;21 Suppl 2:S14-20.

73. Haines A, Jones R. Implementing findings of research. BMJ (Clinical research ed. 1994;308(6942):14881492.

74. Davis DA, Taylor-Vaisey A. Translating guidelines into practice. A systematic review of theoretic concepts, practical experience and research evidence in the adoption of clinical practice guidelines. Cmaj. 1997;157(4):408-416.

75. Taylor G. Changing clinical behaviour. Br J Gen Pract. 2003;53(491):493-494.

76. Shavit I, Keidan I, Hoffmann Y, Mishuk L, Rubin O, Ziv A, et al. Enhancing patient safety during pediatric sedation: the impact of simulation-based training of nonanesthesiologists. Archives of pediatrics \& adolescent medicine. 2007;161(8):740-743.

77. Cravero JP, Havidich JE. Pediatric sedation - evolution and revolution. Paediatric anaesthesia.21(7):800809.

78. Werner C, Smith A, Van Aken H. Editorial Guidelines on non-anaesthesiologists administration of propofol for gastrointestinal endoscopy - a double-edged sword. August 2011. European journal of anaesthesiology. 2011.

79. Kain ZN, Gaal DJ, Kain TS, Jaeger DD, Rimar S. A first-pass cost analysis of propofol versus barbiturates for children undergoing magnetic resonance imaging. Anesthesia and analgesia. 1994;79(6):1102-1106.

80. Leroy PL, Schipper DM, Knape HJ. Professional skills and competence for safe and effective procedural sedation in children: recommendations based on a systematic review of the literature. International journal of pediatrics.2010:934298. 
Appendix

Paediatric sedation: The European experience and approach 


\title{
Pediatric Sedation:The European Experience and Approach
}

\author{
Michael R.J.Sury and Piet L. J.M. Leroy
}

\section{Introduction}

Every system of health care is imperfect because it has limited resources and must cope with increasing demand. Europe has many independent countries and each health service has been influenced by historical, cultural, social, and economic factors. For the management of children having minor diagnostic and therapeutic procedures, there remains considerable variation in practice. Nevertheless, healthcare providers in Europe have been influenced by recommendations from within Europe and the United States (US), and this has led, and will continue to lead, to a general improvement in the quality of services available.

This chapter avoids reiteration of what is commonly known in the United States, and instead is intended to describe and contrast what is different or new in Europe. In doing so, we have drawn upon our personal knowledge, researched the European literature, and gathered some of our own data to describe what we believe to be the important and interesting European problems and perspectives with pediatric sedation.

M.R.J.Sury $(\varangle)$

Department of Anaesthesia, Great Ormond Street Hospital NHS Trust; Portex Unit of Anaesthesia, Institute of Child Health, University College London, London, UK

e-mail: SURYM@gosh.nhs.uk

\section{General Problems}

\section{Demand for Sedation and Anesthesia}

In the last 15 years, the demand for procedures has increased and the availability of anesthesia services has decreased, if not in absolute terms, in proportion to the demand. Five services are prominent and each is discussed in detail. It is reasonable to state that, because of the characteristics of the procedures, each service requires a different sedation strategy and set of techniques. Nevertheless there are similarities in terms of the facilities they need. For specialists planning and negotiating the development of a new service, it may be helpful to consider what facilities are needed. A basic but invaluable list was created by a group of London hospitals who are trying to measure their progress in their compliance with the standards set out in the UK (United Kingdom) Children's National Service Framework (http:// www.ich.ucl.ac.uk/cypph/cnsf_audit_tool.pdf). In a section on Pain, Symptom Relief, and Sedation there are six facilities:

- Analgesia

- Procedural sedation

- Rescue Anesthesia

- Behavioral management (play therapy)

- Long-term central venous access

- Symptom control

All of these will help minimize distress and a comprehensive service should have them. There is debate concerning the pros and cons 
of sedation verses anesthesia but the most important questions are about what happens when anesthesiologists are not available;

1. What drugs are safe enough for nonanesthesiologists to use?

2. What minimal competences and skills should nonanesthesiologists possess to ensure an optimal level of both safety and effectiveness?

Behavioral management is becoming an essential tool, [1, 2] and behavioral skills need to be embedded in training of everyone in the healthcare team- not just Play Specialists and Psychologists. Behavioral management skills help to reduce anxiety and the need for sedation drugs and their value should not be underestimated. Self hypnosis and other coping strategies are useful for cooperative children [3, 4]. Likewise, the early insertion of central intravenous lines avoids many painful venipunctures: interventional radiology services have radically reduced distress in children. There is a wide and strong belief that if children, especially those who need repeated procedures, undergo their first procedure without distress, subsequent procedures are more easily managed and suffering is reduced overall. There is little published evidence for this view.

There are major cultural aspects to the demand for and the practice of sedation. A survey of practice in the US and Europe highlighted major differences in the use of sedation and analgesia for oncology procedures [5] and although the replies may no longer apply, they could be taken as evidence of an acceptance by many children and parents in the US that sedation and analgesia were not necessary for bone marrow aspiration and lumbar puncture. Perhaps the survey was not truly representative, but there is other evidence of cultural behavior having an effect. In France, many painful procedures are undertaken with nitrous oxide alone $[6,7]$, and it is surprising that this practice has not transferred to other countries; probably it is not transferable because patients and parents expect and prefer anesthesia. Nitrous oxide is given without the need for special facilities or fasting, a clear advantage over anesthesia. In the Netherlands, a group of midwives have given birth to infants with major congenital defects. Nitrous oxide was blamed and is no longer available in that country for obstetric analgesia (it is still available for dental sedation). A working group on pediatric procedural sedation is trying to introduce nitrous oxide for procedural sedation but is facing strong opposition.

Also in France, parents are discouraged from remaining with their children during procedures or at induction of anesthesia. In other countries parents are encouraged to be present in many situations, even during resuscitation [8].

There are, within Europe, large differences in choice of sedation drugs. Chloral hydrate is the first-choice drug in the Netherlands for sedation in diagnostic imaging because it has a high safety profile and success rate. In France it has been banned because of suspicion of genetoxicity and carcinogenicity [9].

Physical restraint is a taboo subject. The literature suggests that the application of "straps" in precooperative small children was acceptable in some hospitals or situations in the US [10-12] but perhaps less so in the UK $[13,14]$. There are specific guidelines in the UK for the appropriate use of restraint and which prevents the restraint of an uncooperative child without effective sedation of anesthetic drugs [15]. In Scotland it is illegal to use physical restraint and there are aspects of European Law of Human Rights that prevent restraint also. Several European authors have postulated that procedural restraint is contrary to the Human rights act and the United Nations Convention on The Rights of the Child [16, 17]. The European Association for Children in Hospital states in their charter that avoidance of restraint should be a fundamental part of comfort policy in sick children (http://www.each-for-sickchildren.org). Nevertheless, restraint is still common practice within European pediatric medicine and it is our experience that in general, procedural comfort is not yet considered essential.

\section{Anesthesia Services are Limited}

The following discussion may apply throughout the developed world but is included here to help explain the practice of nonanesthesiologist led sedation. Anesthesia has been developed for 
surgical operations and the development of services outside the operating theater has been slow. Several reasons may explain this. Anesthesia has been developed to provide surgeons with efficient operating lists. Pediatricians, in contrast, have not scheduled their cases in a similar fashion and have not always pressed their need for services. Consequently they have tried to manage on their own with the intention of giving themselves control and responsibility; this has had limited success. Anesthesiologists have been reluctant to help them because resources have not been vouched safe and facilities may not have the standards of operating theaters - at least that was a common perception. There was also a fear of working unsupported at a site remote from other anesthesia colleagues. Given these problems, pediatricians, had no choice but to cope with providing sedation on their own. Anesthesiologists who could help provided anesthesia considered perhaps as unnecessary, out of proportion, higher risk, or more expensive than sedation. Finally, there was an underlying view that once a service was given to pediatricians, it would lead to a considerable increase in demand that would not be possible to satisfy - it was a "bottomless pit." Eventually, with reports of unsafe or ineffective practice, anesthesia services outside theaters have flourished. Today, at least one third of all pediatric anesthetics are given outside surgical operating theaters. Nevertheless there are issues that slow the transition to ready access to good services. We outline them below.

Small hospitals continue to be attractive to the public, who believe that they provide a good service. These units are too small to provide tertiary (specialist) care and possibly unable to provide secondary care if it involves nonstandard techniques in current health services, pediatric care is classified as nonstandard and requires special training. This varies between countries. A small unpublished survey last year showed that in Belgian regional hospitals, most MRI scans in children are done under modern general anesthesia while in the university units, old-fashioned sedation cocktails are still in use because of limited anesthesia resources. In the Netherlands the opposite is true.

Mortality studies of surgery and anesthesia in the UK and elsewhere have identified that the very young and the very old have a higher risk than others [18]. Consequently, this led to specialization and a withdrawal of services to children by anesthesiologists who thought their skills were not sufficient. Some hospitals withdrew pediatric surgery from their services - perversely some Emergency Departments continued to accept pediatric trauma and medical problems that may need anesthesia and intensive care. This remains a common scenario around Europe. Both national as well as European centralization of tertiary care is a problem. Fortunately, the links to larger centers are usually well established and transfer is not difficult although there will be an inevitable delay in treatment. To avoid the need for transfer, some hospitals have developed sedation protocols, mainly ketamine, to help children with minor injuries. A far reaching effect of specialization is the closure of small pediatric units and the expansion of others. This has lead to improvement of services because anesthesia services can be developed economically to deal with larger numbers of cases in dedicated sessions and facilities outside operating theaters.

The European Working Time Directive has limited the hours that doctors can work. It is a statute developed in the EEC to prevent excessive working hours and to encourage more equitable employment. For example, it may be fairer to employ two doctors to work $36 \mathrm{~h}$ per week rather than one for 72; night duty, even if the doctor is in-hospital and asleep, counts as work. This directive, however, is allegedly not applied uniformly across the continent, but in the UK it has severely limited training experience for trainees. Since August 2009, the limit has been set to 48 hours per week.

In 2003, a new UK consultant contract changed the behavior of many consultants. Before 2005, most consultants (nontrainees) worked sessions and provided services that were not fixed nor agreed by contract. Such an unclear system of employment was vulnerable to criticism of ineffective management and this persuaded the politicians to demand clear agreement and contracts. Now, work is fixed by contract. However, this does not seem to have increased patient throughput but it may have encouraged improvements in efficiency. Yet, part of the debate has been about 
quality of services rather than quantity. A system of fee for session and, as in the US, fee for service, limits flexibility and prevents natural changes in service. If a pediatrician wants a sedation service, and asks for anesthesiologists to provide it, will he deliver anesthesia rather than sedation? Reimbursement based on service can have perverse outcomes, such as preventing the use of simple effective techniques in preference to financially advantageous anesthesia. Another problem relates to the case throughput. If payment is too low there is incentive for fast techniques that may not be safe or effective. Mindful of these problems, the payment by salary unrelated to number or complexity of cases, allows the practitioner to provide a service tuned to the needs to the patients.

In France, preoperative assessment by an anesthesiologist is compulsory, by law, at a minimum of $24 \mathrm{~h}$ before any routine procedure. This has restricted the involvement of anesthesia services in the delivery of sedation or minimal anesthesia for children and encouraged the use of nitrous oxide alone by nonanesthesiologists.

\section{Nonanesthesia Practitioners}

In the UK and much of Europe, anesthesia is a physician led service. In Scandinavian countries and the Netherlands, nurses are employed to assist physicians; they look after patients during surgery but they are supervised by physicians and not by surgeons. This system may develop in the UK but, because there is a surplus of trained anesthesiologists, it is not likely to grow significantly in the foreseeable future. In pediatric anesthesia, almost all anesthesia services throughout Europe are physician led.

Because of the scarcity of pediatric anesthesiologists, several professional groups have had to use drug techniques that have the potential to become accidental anesthesia. The dentists, emergency physicians, and intensivist have been prominent. Their journey, from inexperienced sedationist to practitioner with proven but limited anesthesia skills, has not reached its end. It is inevitable that they must continue in the venture to provide effective and safe services for their patients. Once rigorous competences, skills, and safety precautions have been fulfilled, nonanesthesiologists in Europe have been given access to potent sedatives (e.g., Propofol) [19, 20]. However, this is as controversial in Europe as it is in the US: [21].

\section{Challenges and Setbacks}

Safety issues, adherence to guidelines, and the training and skills of the sedation provider have been of recent concern in Europe. Three cases with disastrous outcomes have attracted widespread notoriety and press in Europe.

- A child's brain was damaged by $100 \%$ nitrous oxide given from an anesthetic machine that did not have a hypoxic mixture alarm. The practitioner was untrained in its use.

- A child died after being suffocated by a team trying to use a breathing system to deliver a nitrous oxide/oxygen mixture because they failed to turn the gas flow on. They were untrained.

- A combination of midazolam alfentanil and ketamine was given to sedate a boy for dental extractions. He became apneic soon after arrival in the recovery area and, neither the nurse nor the doctor reacted quickly enough to prevent permanent hypoxic brain damage [22]. Lack of sufficient training was the prominent issue with these cases and although it is tempting to think that anesthesiologists would not have made those mistakes, it is important to accept that every professional is vulnerable to human error. The doctor in the dental sedation disaster was an anesthesiologist.

In the Netherlands there have been three severe accidents in the last decade ( 2 with a fatal outcome and 1 with permanent neurological damage) in hospitalized children during sedation for MRI scanning. In all cases, sedation was provided by nonanesthesiologists, using combinations of long-acting sedatives. Health Inspectorate's investigation clearly showed that existing safety guidelines were not implemented in these cases. The question rose whether these 
were isolated incidents. Subsequently, adherence to safety guidelines on pediatric procedural sedation in all hospitals in the Netherlands was investigated; adherence was not high and was unsatisfactory [23]. A nationwide survey of pediatricians queried their adherence to Pediatric Sedation (PS) safety guidelines. These guidelines were divided into presedation assessment, monitoring during PS, recovery and facilities, and competencies for emergencies and rescue. Pediatricians from 88 of the 97 Dutch hospitals responded. Less than $25 \%$ of respondents adhered fully to safety guidelines [24].

In a pilot survey among European pediatric anesthesiologists, we have found that similar accidents have happened elsewhere although none have been published. The exact characteristics of sedation practices by nonanesthesiologists have not been studied systematically but we believe that unsafe practice is still widespread [25].

\section{Monitoring}

Capnography and level of consciousness monitoring are probably less frequently used in Europe as compared to the United States. Capnography is useful, that cannot be denied, but probably its general use in sedated patients may not be widespread. A study from Turkey promotes its value in maintaining safety [26]. Limitations to its adoption have included limited financial resources. BIS and other monitors are scarcely used in the operating rooms for children; yet, they do have a place in the management of children who cannot tolerate standard anesthesia [27].

\section{Recommendations}

Anesthesiologists throughout the world have been quick to state the problems of sedation by the untrained and have published guidelines to prevent disasters. Excluding dentistry, the UK guidelines focused first on the Radiology setting [28] and then in 2001 the Academy of Medical Colleges responded to reports of unacceptable mortality in adult patients having esophago-gastroscopy [29].
They stated clearly, that "organizations should ensure that staff receive sedation training." The Scottish Intercollegiate Guidelines Network [30] gathered a body of opinion from across many specialties and developed a clinical guideline that has been quoted and used widely. In Italy, a fine review and guideline was produced for pediatric neuroradiology [31]. A guideline for nonanesthesiologists has been published for application throughout Europe [32]. However, in our own survey most respondents were not aware of any national or European guideline. National guidelines are available in the UK, Netherlands, and France.

Had any of these guidelines been applied, the aforementioned disasters would not have happened. Although these guidelines may have already, prevented many catastrophes, in the authors' opinion they would benefit from endorsement and dissemination by the specialty organizations. The dentists have progressed the most in sedation management and their efforts are discussed later. Capnography, properly applied, would have warned of a respiratory problem and may have avoided fatal outcomes.

\section{Definitions}

Initially, conscious sedation was an accepted endpoint or landmark in the continuum of conscious level. Conscious, meaning able to respond to the spoken word, has been replaced by the term moderate sedation in the current literature because it does not assume consciousness but rather that the patient is easily roused - usually by communication but also by other similar appropriate light stimulus [33]. Nevertheless, conscious sedation remains a common term $[28,34]$. In the UK, dentists prefer the term conscious sedation because they define this as a level of sedation at which the patient responds easily to commands rather than any other stimulus.

The term deep sedation was not approved [28] and still is not in some professional groups, because it was indistinguishable from anesthesia. While this point may be overstated, it has led to the recommendation that both deep sedation and anesthesia must be managed by the same personnel, 
equipment, and facilities. The definition therefore becomes more useful as a description of the intended conscious level rather than as a division on the basis of resources or risk. In a similar desire, two other descriptions of deep sedation/anesthesia have been used. Light anesthesia [35] or minimal anesthesia [36] are terms that could be used to describe a technique in which the patient seems unconscious although any appreciable stimulation is likely to rouse them. Propofol or sevoflurane [37] have been used to provide conditions with sufficient immobility for painless imaging.

Dissociative sedation is not a term in common use, but it is understood. Ketamine sedation or anesthesia is preferred generally.

Relative analgesia (RA) is a term intended to describe the analgesia and mild euphoria and calming properties of $30 \%$ nitrous oxide. Dentists have become expert in its use [38].

The question remains how well these definitions reflect reality and to what extent the outcome level can be predicted, especially when non-titratable drugs are used. These questions are relevant since procedural sedation by nonanesthesiologists is often performed using long-acting, nonintravenously administered medications. Motas showed that common drugs (e.g., chloral hydrate, midazolam, pentobarbital) in average doses cause wide variations in depth of sedation [39]. The goal of either conscious or deep sedation was not achieved in a significant number of children. Considering sedation levels as a sliding scale, rather than a step-by-step decline of consciousness, the Dutch working group on Procedural sedation decided to define in their new evidencebased guideline the same safety precautions for all levels beyond anxiolysis/mild sedation (www. cbo.nl).

\section{Training and Credentialing}

With the exception of dental sedation, there are no national training programs or qualifications for sedation. It is difficult to design a universal training schedule for the many different types of sedation, some of which will not be relevant for specialists. Four strategies that could move us towards credentialing have been clearly identified by Krauss and Green. [40] We favor the option of creating a safe and effective service controlled by the institution who takes their direction from national and professional guidelines. Such a system should bring development of efficient training that may evolve into national training schedules.

A seemingly straightforward skill that all sedationists should have is airway management and resuscitation. Access to live patients is a limiting factor and the development of life-like manikins is a potential solution. European resuscitation courses are widespread but do not aim to teach the monitoring and proactive airway skills that sedationists need. This should be a common component of specialty-specific sedation training courses.

\section{Implementation}

Several implementation factors separate Europe from the US. European standards of practice are mainly enforced by professionals themselves, whereas in the US the aspirations of professionals are enforced by financial penalty by insurance companies who demand that standards are maintained. In the UK, the National Institute of Clinical Excellence and Healthcare are producing guidelines for specific clinical problems and these will be enforced by government directive as well as by financial penalty to Hospitals. Clinical Governance is a term applied in the UK NHS to force individuals to bear responsibility for their actions and make sure that someone is accountable for failings in the service; it has helped improve quality and safety.

The number of malpractice actions is reputed to be highest in the US and the threat of financial loss and public distrust has been a driver for change. The publication of the US closed claims analyses has been very helpful and although defense organizations publish case studies and recommendations, there is nothing in that scale available in Europe.

In the Netherlands, and elsewhere, the implementation of guidelines on Procedural Sedation 
and Analgesia (PSA) has been encouraged by raising public awareness through media and charities.

\section{Common European Sedation Practice for Selected Procedures}

\section{Radiology}

\section{Painless Imaging}

Both continents have tried to maximize the use of sedation for painless imaging. Nurse-led services for example were promoted as a practical alternative to anesthesia [41, 42]. Chloral hydrate [43] or Triclofos [44] have been the mainstay for children under $15 \mathrm{~kg}$ and have very good safety and success records; safety depends upon the user more than the drug; $95 \%$ of children fall asleep within one hour and remain asleep for approximately $45 \mathrm{~min}$. In older children, few drugs are as effective, leading most hospitals to abandon sedation in this group [45]. Pentobarbital was withdrawn in the UK in the 1960s due to its potential for abuse. Secobarbital has been used but causes paradoxical reactions (as in pentobarbital). Dexmedetomidine, although not widely available in Europe was trialed in Turkey [46, 47]. So-called lytic cocktails are still commonly in use in the Netherlands.

The unreliable nature of sedation has caused many, if not most, hospitals to develop anesthesia led services [48] because there is a general acceptance that anesthesia is more efficient and maybe safer [49]. Certainly propofol [50] and sevoflurane [37] are standard techniques that are compatible with rapid recovery to street-fitness. Propofol may need to be combined with other drugs to maintain immobility and recently a combination of midazolam, nalbuphine and low dose propofol has been found to be reliable [51].

\section{Interventional Radiology and Cardiology}

Many intravenous lines can be inserted with a combination of moderate sedation and behavioral techniques; however, this requires appreciable effort to select children who can tolerate this course. Ketamine may be an alternative technique but we believe that interventional radiology is more readily managed by an anesthesia service because of its flexibility and the ability to overcome almost any problem. For cardiology some countries have managed to maintain an effective sedation service using a range of techniques involving combinations of propofol [52], ketamine [53], and remifentanil [54], but our view is that the practice of controlled ventilation using tracheal intubation and standard anesthesia techniques is more reliable and creates optimal conditions for imaging and measurements [27, 55].

\section{Gastroenterology}

We believe that many hospitals in Europe use sedation for endoscopy with a combination of benzodiazepines and opioids [56]. Surveys in both the Netherlands and the UK showed that $50 \%$ of endoscopies in nonuniversity hospitals are performed under this regimen. If there have been few problems, this is a credit to the judgment of gastroenterologists because the literature suggests that sedation is difficult especially for esophagoscopy [57]. It is likely that most practitioners prefer anesthesia [58]. An exciting development for gastroenterologists is the use of propofol without tracheal intubation for upper and lower endoscopies [45]. Some anesthesiologists are confident that this is a safe approach $[19,45,59,60]$ provided the gag reflex is not completely suppressed during upper endoscopy; lower endoscopy needs much less propofol except when the ascending colon, the cecum, and the terminal ileum are entered (a small dose of opioid may be useful at these times). Not only is this technique a reliable and safe alternative to benzodiazepine-based sedation, but it radically increases the patient throughput. In financial terms, this technique seems unbeatable. However, there may be many circumstances when it is not appropriate and many anesthesiologists believe that a technique involving tracheal intubation remains the safest of all. 
Propofol, remifentanil, and desflurane could be used in a technique that is equally rapid (especially for colonoscopies).

\section{Oncology}

Many techniques are possible for children who need repeated painful oncology procedures. With practice, nitrous oxide alone is potentially useful. In most countries we believe that intravenous anesthesia is preferred [61]. Without anesthesia services, ketamine is a reliable technique. The addition of a short acting opioid to propofol is probably a common technique because it reduces the dose of propofol. Propofol with remifentanil has the potential to provide the most rapid technique. The apnea that it can cause indicates that the child will remain immobile during the procedure, albeit with assisted ventilation [62].

\section{Emergency Medical Care}

Procedural sedation and analgesia is being developed and applied on both sides of the Atlantic. There seems to be a gradual but steady progression by Emergency Physicians to develop their own standards and protocols such that in Europe and in the US, hospitals support the use of ketamine [63], opioids, and propofol to manage children for minor procedures. There may be a trend for emergency departments becoming focused on quality and safety. However, PSA is currently not incorporated in European training programs. A recent European study showed that in most Pediatric Emergency Departments (PED), PSA is practiced to the level of mild to moderate sedation. In about $20 \%$ of the PEDs deep sedation is not provided by the staff, while $7.5 \%$ of departments had no PSA available for their patients [64].

Alternatively, some hospitals have made extra efforts to provide anesthesia services, usually at fixed times of the day, to meet maximum demand [65]. In the UK, a ketamine protocol has been produce by the College of Emergency Physicians
(http://www.collemergencymed.ac.uk/CEC/cec_ ketamine.pdf); it is clear and explicit.

\section{Dentistry}

Dentists were pioneers of sedation and many are expert in their practice. They know that during conscious (moderate) sedation the patient should be rousable by verbal command but in addition they have observed that the mouth closes during deeper sedation. To keep the mouth open is a voluntary action and therefore mouth closure warns the dentist of a potential problem with the airway. It is important therefore to not use a mouth prop to keep the mouth open during sedation. Effective local anesthesia should make sedation much easier [66] yet many patients are fearful of the pain of needles in the mouth. For patients who will not, despite all behavioral techniques, accept the insertion of local anesthesia, sedation deeper than mild sedation is probably necessary. Mild sedation rarely, if ever, changes a yes to a no.

Nitrous oxide relative analgesia (RA) has been popular because it is remarkably safe and surprisingly well tolerated by children [67]. Dental "gas" machines are designed with devices to protect the patient against hypoxic gas mixtures and the breathing system connects to a nasal mask from which scavenging is possible. In children who tolerate nitrous oxide, gas mixtures with less than $30 \%$ nitrous oxide are almost always effective. More than this causes dysphoria, dizziness, and nausea [38]. Recommendations accept that hypoxia is so unlikely that pulse oximetry and fasting are unnecessary (large meals beforehand are discouraged however) [68]. Nitrous oxide given in a 1:1 mix with oxygen has been used in many children for a variety of procedures [6]. Hypoxia was rare, as was any airway obstruction and these problems only occurred when the patient had a cerebral disorder or was having another sedative drug [7]. Furthermore, in obstetric practice, fasting and pulse oximetry are not required during nitrous oxide analgesia (although nitrous oxide is self administered via a demand valve in contrast to the free flow apparatus used in Belgium and France). 
Standard sedation for children is limited to RA in most parts of Europe [69]. When nitrous oxide is insufficient to calm a patient, other drugs have been added. These may tip the patient into deep sedation, which is an obvious hazard, even though the risk may be small. In a study comparing RA with a combination of RA and $0.1-0.3 \%$ sevoflurane, the dental treatment was completed in $52 \%$ and $89 \%$, respectively. The same team, in another study, found that sevoflurane $(0.3 \%)$ added to nitrous oxide $(40 \%)$ and intravenous midazolam was effective in 93\% (249/267) of anxious children who would have been given general anesthesia otherwise [70]. All children remained rousable and none required airway management or oxygen - nevertheless, all children were fasted and monitored and these techniques were delivered by trained anesthesia personnel in a specialist dental clinic.

Other dentists have tried oral drugs. Oral and rectal benzodiazepines are commonplace in Sweden [71]. Midazolam is often useful to calm children [72] but treatment may have to be limited to minor restorations only [73]. In uncooperative toddlers (2-4-year old) a cocktail of chloral hydrate, meperidine, and hydroxyzine was effective in only $72 \%$ and adverse conditions including vomiting, desaturation, prolonged sedation, and an apneic event occurred in $3 \%$ of all sedations (but were reported as minor) [74].

Intravenous midazolam alone is recommended in the UK for anxiolysis in children over 16 [69] and may be appropriate and effective in younger adolescents [75]. Propofol has been used alone as a sedation technique but lacks the analgesic component to enable insertion of local anesthesia [76]. Consequently, intravenous cocktails containing midazolam, alfentanil, ketamine, and propofol are being explored [77, 78]. A recent review of experience in 1,000 cases shows that these drugs can be combined safely [79]; loss of verbal contact occurred in approximately $0.05 \%$ and nausea was a problem in 5\%. Whether this "alternative" technique can be called sedation is debatable if it is unknown whether it will cause accidental anesthesia. Certainly, alfentanil can cause apnea when the pain of dental treatment has subsided [22].
Many of these specialist techniques may not be applicable outside specialist centers and there is some evidence to support the view that most dentists and anesthetists believe that uncooperative children should be managed with short acting anesthesia in a hospital setting [80, 81]. Recently, in the UK, a group of dentists have pressed for conscious sedation techniques to progress beyond the limits of RA (and benzodiazepines for adolescents). They now have recommendations to develop new sedation techniques using subanesthetic doses of potent anesthesia drugs. Time will show how safe these techniques are.

\section{New and Future Developments}

Training and accreditation are the most important objectives for sedationists around the world. Their skills need to be focused on the type of sedation that they need to administer and their protocols will need to restrict their practice to avoid unexpected problems. We believe that airway management and monitoring skills should be generic to any qualification.

A new guideline - Sedation for diagnostic and therapeutic procedures in children and young people - has been developed in the UK and published by NICE in December 2010 [82, 83]. NICE is the National Institute for Health and Clinical Excellence of the UK. These guidelines incorporated evidence of safety and efficacy of selected sedation drugs, consensus statements about patient management, and cost effectiveness considerations. Important deviations in these guidelines from those of the United States are the recognition of propofol and sevoflurane inhalation as agents appropriate for pediatric sedation [82] (Table 17.1). This NICE guideline is unique among other NICE guidelines because it specifies the principles of training needed to use effective sedation techniques safely. It states that healthcare professionals trained in the delivery of anesthesia may administer sevoflurane, propofol, or a combination of opioids with ketamine. A treatment pathway and sedation algorithm is detailed in Fig. 17.1 [82]. 
Table 17.1 Current licensing status for sedation drugs* (NICE Guidelines)

\begin{tabular}{|c|c|c|}
\hline Drug & Indication & $\begin{array}{l}\text { Licensed use (taken from the British National } \\
\text { Formulary for children }(B N F c) \text { 2010/1133) }\end{array}$ \\
\hline Chloral hydrate & For mild to moderate sedation & $\begin{array}{l}\text { Not licensed for sedation in painless procedures. For } \\
\text { dosing (by mouth or by rectum) for painless } \\
\text { procedures in children from neonates to } 18 \text { years, see } \\
\text { the } B N F C\end{array}$ \\
\hline \multirow[t]{2}{*}{ Fentanyl } & $\begin{array}{l}\text { For analgesia and for improved } \\
\text { anesthesia }\end{array}$ & $\begin{array}{l}\text { Licensed for use in children older than } 1 \text { month with } \\
\text { spontaneous respiration for analgesia, and during } \\
\text { operations for improved anesthesia by intravenous } \\
\text { injection over at least } 30 \text { seconds }\end{array}$ \\
\hline & For moderate to deep sedation & $\begin{array}{l}\text { If deep sedation is needed. a general anesthetic (e.g., } \\
\text { propofol or ketamine) or a potent opioid (e.g., } \\
\text { fentanyl) may be used; these should be used only } \\
\text { under the supervision of a specialist experienced in } \\
\text { the use of these drugs }\end{array}$ \\
\hline Intranasal diamorphine & $\begin{array}{l}\text { For mild to moderate sedation } \\
\text { in managing acute pain and short } \\
\text { painful procedures }\end{array}$ & $\begin{array}{l}\text { Licensed for intranasal route but listed in the } B N F c \text { as } \\
\text { follows: acute pain in an emergency setting or short } \\
\text { painful procedures; intranasally in children heavier } \\
\text { than } 10 \mathrm{~kg}\end{array}$ \\
\hline \multirow[t]{2}{*}{ Ketamine } & Anesthesia & $\begin{array}{l}\text { Licensed for use in anesthesia for all ages; intrave- } \\
\text { nous and intramuscular }\end{array}$ \\
\hline & $\begin{array}{l}\text { Lower doses are used } \\
\text { for moderate sedation }\end{array}$ & $\begin{array}{l}\text { If deep sedation is needed, a general anesthetic (e.g., } \\
\text { propofol or ketamine), or a potent opioid (e.g., } \\
\text { fentanyl) may be used. However, they should be used } \\
\text { only under the supervision of a specialist experienced } \\
\text { in the use of these drugs }\end{array}$ \\
\hline \multirow[t]{4}{*}{ Midazolam } & \multirow{4}{*}{$\begin{array}{l}\text { For mild to moderate } \\
\text { (also referred to as conscious) } \\
\text { sedation }\end{array}$} & $\begin{array}{l}\text { Not licensed for use in children younger than } 6 \\
\text { months for premedication and conscious sedation }\end{array}$ \\
\hline & & $\begin{array}{l}\text { Not licensed for use by mouth or by buccal } \\
\text { administration }\end{array}$ \\
\hline & & $\begin{array}{l}\text { Intravenous midazolam is not licensed for use in } \\
\text { children younger than } 6 \text { months for conscious } \\
\text { sedation }\end{array}$ \\
\hline & & $\begin{array}{l}\text { No UK marketing authorization for oral or intranasal } \\
\text { midazolam for sedation. However, dosing for children } \\
\text { from age } 1 \text { month is given in the } B N F c\end{array}$ \\
\hline Morphine & Analgesia and for deep sedation & $\begin{array}{l}\text { Licensed for analgesia in all ages; subcutaneous or } \\
\text { intravenous. Other routes have restricted licensing; } \\
\text { Oramorph solution (morphine) is not licensed for use } \\
\text { in children younger than } 1 \text { year; Oramorph unit dose } \\
\text { vials is not licensed for use in children younger than } 6 \\
\text { years; Sevredol tablets (morphine) are not licensed for } \\
\text { use in children younger than } 3 \text { year; MST continuous } \\
\text { preparations (slow release morphine sulfate) are } \\
\text { licensed to treat children with cancer pain (age range } \\
\text { not specified by manufacture); MXL capsules } \\
\text { (morphine) are not licensed for use in children younger } \\
\text { than } 1 \text { year). If deep sedation is needed, a general } \\
\text { anesthetic (e.g., propofol or ketamine) or a potent } \\
\text { opioid (e.g., fentanyl) may be used; these should be } \\
\text { used only under the supervision of a specialist } \\
\text { experienced in the use of these drugs }\end{array}$ \\
\hline
\end{tabular}


Table 17.1 (continued)

\begin{tabular}{|c|c|c|}
\hline Drug & Indication & $\begin{array}{l}\text { Licensed use (taken from the British National } \\
\left.\text { Formulary for children }(B N F c) 2010 / 11^{3}\right)\end{array}$ \\
\hline Nitrous oxide & $\begin{array}{l}\text { For minimal to moderate sedation } \\
\text { during relatively short procedures }\end{array}$ & $\begin{array}{l}50 \% \text { nitrous oxide licensed for use in sedation for all } \\
\text { ages (inhalation); nitrous oxide in concentrations }> \\
50 \% \text { is not licensed for analgesia without loss of } \\
\text { consciousness }\end{array}$ \\
\hline Opioids & For moderate to deep sedation & $\begin{array}{l}\text { If deep sedation is needed, a general anesthetic } \\
\text { (e.g., propofol or ketamine) or a potent opioid } \\
\text { (e.g., fentanyl) may be used; these should be used } \\
\text { only under the supervision of a specialist experienced } \\
\text { in the use of these drugs }\end{array}$ \\
\hline \multirow[t]{3}{*}{ Propofol } & Anesthesia & $\begin{array}{l}\text { Licensed for use in all children older than } 1 \text { month in } \\
\text { intravenous doses of } 0.5 \% \text { or } 1 \%\end{array}$ \\
\hline & \multirow[t]{2}{*}{ For moderate to deep sedation } & Licensed for use in people older than 17 years \\
\hline & & $\begin{array}{l}\text { The Guideline Development Group decided to } \\
\text { recommend off-label use of propofol for sedation in } \\
\text { children of all ages. This was because propofol is } \\
\text { widely used in the UK for sedation in children of all } \\
\text { ages and the doses used for sedation are much lower } \\
\text { than those used for anesthesia. If deep sedation is } \\
\text { needed, a general anesthetic (e.g., propofol or } \\
\text { ketamine) or a potent opioid (e.g., fentanyl) may be } \\
\text { used; these should be used only under the supervision } \\
\text { of a specialist experienced in the use of these drugs }\end{array}$ \\
\hline \multirow[t]{2}{*}{ Sevoflurane } & Anesthesia & Licensed for use in anesthesia for all ages (inhalation) \\
\hline & For moderate to deep sedation & Sedation is outside the licensed use \\
\hline
\end{tabular}

* These drugs have been recommended for pediatric sedation. Informed consent should be obtained and documented for the use of any drug outside the licensed indications

Source: Reproduced from Sury et al. [82], with permission from BJM Publishing Group Ltd

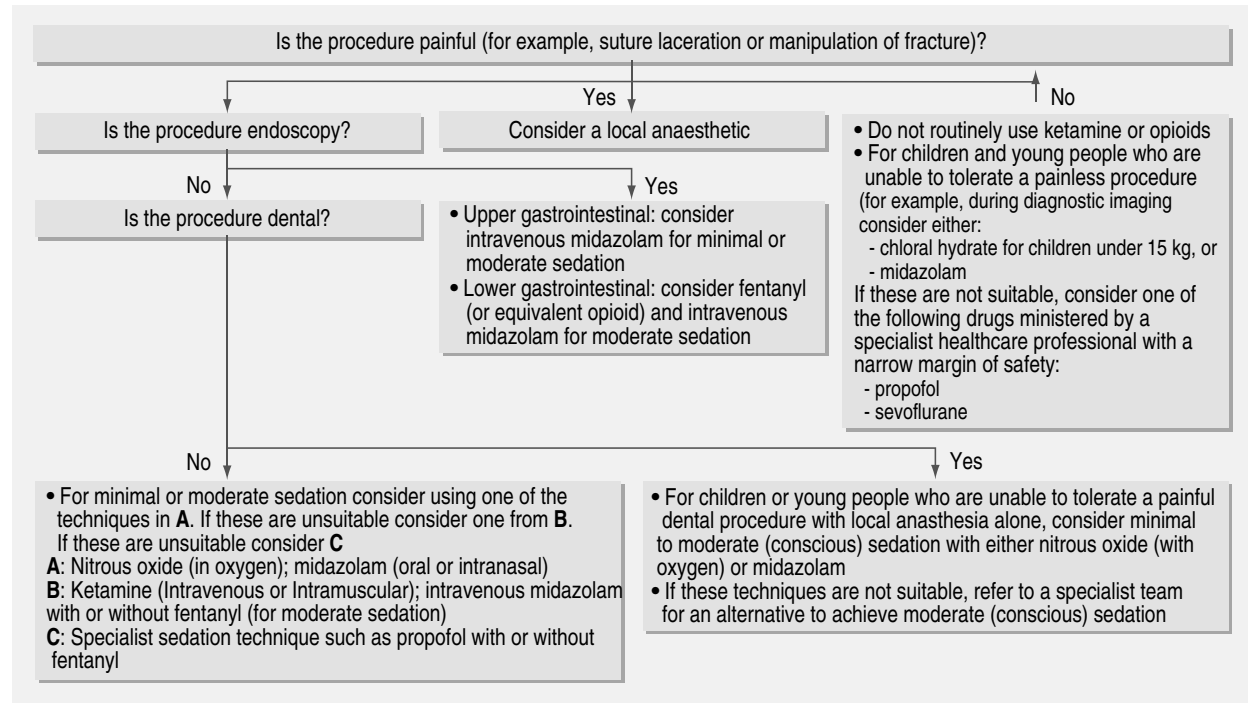

Fig. 17.1 Sedation algorithm and pathway (reproduced from Sury et al. [82], with permission from BJM Publishing Group Ltd) 
In the Netherlands, the Dutch Institute for Healthcare Improvement (CBO) commissioned Pediatric Guidelines for Procedural Sedation and/ or Analgesia (PSA) at Locations Outside the Operating Theatre from the Netherlands Society of Anesthesiologists and the Dutch Society of Pediatrics [84]. Recently published in 2011, the Guidelines were meant to represent six important cornerstones, notably including the optimal use of local or topical anesthesia, nonpharmacological techniques, and the prohibition of forced securing and restraint [84] (Table 17.2).

These Dutch guidelines were noteworthy because they distinguished deep sedation from dissociative sedation [84] (Table 17.3). Sedation of ASA III and IV patients by nonanesthesiologists

Table 17.2 Cornerstones of a comprehensive policy towards procedural comfort in Children, Dutch Institute for Healthcare Improvement

1. Prevention of procedural pain and stress

2. An active policy in relation to the prevention of forced securing and restraint

3. Optimal use of effective forms of local or topical anesthesia

4. The systematic application of effective non-pharmacological techniques (preparation, distraction, hypnosis, etc.)

5. The application of the most adequate PSA technique, individually titrated and carried out by a trained professional

6. A local policy towards the ready availability of the so-called "rescue anesthesia" if a PSA technique turns out to be inadequate or if it can be anticipated that the available PSA techniques may be insufficient or unsafe in an individual patient

Source: Reproduced with permission from [84]. Table 17.1. Note: The final version of the guidelines is pending approval by the Dutch Society of Pediatrics and the Dutch Society of Anesthesiology

Table 17.3 Definitions of different levels of sedation, Dutch Institute for Healthcare Improvement

1. Light sedation/anxiolysis: Two states that are difficult to tell apart, in which the anxiety and stress level of the patient have been lowered while the patient remains basically fully conscious. The patient responds adequately and consistently to verbal stimuli, and verbal communication therefore remains possible. This state is associated with few risks in patients without significant comorbidity. Although cognitive functions and coordination are reduced, ventilatory and cardiovascular functions remain unaffected. Light sedation/anxiolysis is typically a state of mind that occurs after 1 standard dose of midazolam $(0.1 \mathrm{mg} / \mathrm{kg}$ intravenously or $0.2-0.5 \mathrm{mg} / \mathrm{kg}$ transmucosally) and with nitrous oxide sedation (inhalation concentration up to 50\%). Higher doses, other medicines, and combinations with other analgesics will virtually always lead to a deeper sedation level

2. Moderate sedation: Pharmaceutically induced reduction in awareness, during which the patient still responds purposefully when spoken to, or to light tactile stimuli. In this stage, no interventions are needed to keep the airway open, airway reflexes are intact, and ventilation is adequate. If the response is not clearly adequate and purposeful but more of a withdrawal reflex, we speak of deep sedation

3. Deep sedation: This is a pharmaceutically induced decline in awareness, during which the patient does not respond to being spoken to, but reacts purposefully to repeated or painful stimuli. Airway reflexes and ventilation may be reduced and it may be necessary to keep the airway open. The concept of "deep sedation" is a contested term because the distinction with anesthesia becomes less clear. A typical example is the deep sedation caused by propofol, during which it is possible, with the necessary expertise, to keep spontaneous respiration going and the airway open. The risk of reduced breathing is more or less a linear function of the dose and depth of sedation

4. Dissociative sedation: Also called a trance-like cataleptic sedation, it is typically the result of sedation with ketamine. As far as the depth of sedation, analgesia, and response level is concerned, ketamine causes a state that primarily corresponds to anesthesia. However, contrary to anesthesia, the airway reflexes, respiration, and hemodynamics largely remain intact, even at comparatively high doses. It makes ketamine attractive for use in PSA, particularly for painful procedures

5. General anesthesia: A pharmaceutically induced state of unconsciousness, in which the patient is unresponsive, even to painful stimuli. The ability to keep the airway open will often be reduced or absent, and ventilation will frequently be depressed, consequently requiring support. Cardiovascular functions may also be impaired. Can only be applied under the personal supervision of an anesthesiologist

Source: Reproduced with permission from [84]. Table 17.2. Note: The final version of the guidelines is pending approval by the Dutch Society of Pediatrics and the Dutch Society of Anesthesiology 
is discouraged and, if performed, should be done only after consultation with an anesthesiologist and by a specially trained and credentialed nonanesthesiologist. Fasting status (NPO) deviates from guidelines of other specialty societies in that light sedation does not need NPO status. An emergent, acute condition in a child who does not have an empty stomach is not an absolute contradiction for PSA [84] (Table 17.4).

Propofol, in the Dutch guidelines, although preferably administered by an anesthesiologist, may be delivered, by an experienced nonanesthesiologist for ASA I and ASA II patients. Patients of ASA III status and higher can only receive propofol from an anesthesiologist [84] (Table 17.5). These guidelines are unique in that they have specific recommendations which are procedure based: Gastrointestinal procedures in particular should favor propofol, if necessary in combination with midazolam or an opioid [84] (Table 17.6).

It is hoped both the NICE and Dutch initiatives will be a fresh attempt to consider the evidence about effective and safe sedation for children and that their output will further encourage an improvement in the services available to children in Europe and beyond.

Table 17.4 NPO fasting recommendations, Dutch institute for healthcare improvement

1. Fasting is not needed for children undergoing light sedation

2. A child must preferably have an empty stomach for any (elective) PSA with moderate or deep sedation, in accordance with the same guidelines that apply to interventions taking place under general anesthesia (two hours for clear liquids, four hours for breastfeeding, and six hours for other meals)

3. A child in an acute condition without an empty stomach is in itself no absolute contra-indication for PSA. This is important if postponing the procedure would pose health risks and/or discomfort. However, in that case the choking risks must always be carefully considered, taking into account the choice of sedative, the depth of sedation, and any protection of the airway. In practice, this amounts to the following recommendations

(a) With PSA in an acute situation (without an empty stomach), deep sedation must be avoided as much as possible, since the protective airway reflexes may be disturbed or there is a high risk of respiratory impairment

(b) If a procedure requires a form of deep sedation, the patient must have an empty stomach

(c) If a procedure requiring a form of deep sedation is urgently needed and an empty stomach can therefore not be guaranteed, deep sedation must performed under the supervision of an anesthesiologist in order to ensure optimal protection of the airway

4. Not having an empty stomach must be no reason or excuse for performing a procedure with an ineffective form of light or moderate sedation

Source: Reproduced with permission from [84]. Recommendation 10. Note: The final version of the guidelines is pending approval by the Dutch Society of Pediatrics and the Dutch Society of Anesthesiology

Table 17.5 Propofol recommendations, Dutch institute for healthcare improvement

Propofol is suitable for application in (urgent) painful procedures in children. Propofol causes deep sedation to anesthesia. The preconditions on patient selection, skills, competencies, monitoring, and the other preconditions set out in part I of this guideline must therefore be complied with. Since propofol is a fast-acting, very potent medicine that can quickly lead to oversedation and respiratory depression in untrained hands, the working group also has the following recommendations:

1. The person who performs the PSA must never be the same person as the one carrying out the procedure or intervention

2. The PSA is preferably carried out by an anesthesiologist

3. If the PSA with propofol is carried out by a nonanesthesiologist, it must be performed by a physician who has already been working with the medicine for a longer period of time and who is able to assess and deal with any respiratory complications

4. PSA with propofol in patients of ASA class III or higher must be performed by an anesthesiologist

5. Preoxygenation and monitoring through capnography with PSA using propofol is strongly encouraged in order to restrict the comparatively high risk of respiratory complications

Source: Reproduced with permission from [84]. Note: The final version of the guidelines is pending approval by the Dutch Society of Pediatrics and the Dutch Society of Anesthesiology 
Table 17.6 Sedation recommendations for GI procedures, Dutch institute for healthcare improvement

1. A gastrointestinal (GI) endoscopic examination in a child must be carried out in principle under general anesthesia or deep sedation. If it is decided to opt for deep sedation, then titratable medicines must be used that are certain to lead to an effective level of deep sedation. Of all the medicines studied, propofol is the most effective, if necessary in combination with midazolam or an opiate

2. The working group advises against the following forms of PSA for GI endoscopic examinations:

Using ketamine for endoscopic examinations of the esophagus, stomach, and duodenum, since there is an increased risk of laryngospasm

Using a benzodiazepine on its own or the combination of benzodiazepine with an opiate. Both forms of PSA are substantially less effective than anesthesia or deep sedation with propofol

Benzodiazepines must not be considered as suitable medicines to generate a reliable level of amnesia for endoscopic procedures

3. As far as rectoscopies are concerned, it is worth contemplating whether the investigation could be carried out without PSA insofar as informed consent has been obtained and provided the child is not scared or opposed to the examination

4. If general anesthesia or the support of an anesthesiologist are not feasible, an endoscopic department must have access to the logistic possibilities as well as trained professionals in order to provide safe and effective deep sedation that fulfills the preconditions of these guidelines

5. Premedication with midazolam taken orally can be considered prior to deep sedation. It reduces stress levels for inserting the drip at the start of the procedure and may therefore result in a smaller dose of propofol being required

Source: Reproduced with permission from [84]. Note: The final version of the guidelines is pending approval by the Dutch Society of Pediatrics and the Dutch Society of Anesthesiology

\section{References}

1. Pressdee D, May L, Eastman E, Grier D. The use of play therapy in the preparation of children undergoing MR imaging. Clin Radiol. 1997;52(12):945-7.

2. Armstrong TS, Aitken HL. The developing role of play preparation in paediatric anaesthesia. Paediatr Anaesth. 2000;10(1):1-4.

3. Liossi C, White P, Hatira P. Randomized clinical trial of local anesthetic versus a combination of local anesthetic with self-hypnosis in the management of pediatric procedure-related pain. Health Psychol. 2006;25(3):307-15.

4. Liossi C, Hatira P. Clinical hypnosis in the alleviation of procedure-related pain in pediatric oncology patients. Int J Clin Exp Hypn. 2003;51(1):4-28.

5. Hain RD, Campbell C. Invasive procedures carried out in conscious children: contrast between North American and European paediatric oncology centres. Arch Dis Child. 2001;85(1):12-5.

6. Annequin D, Carbajal R, Chauvin P, Gall O, Tourniaire B, Murat I. Fixed 50\% nitrous oxide oxygen mixture for painful procedures: a French survey. Pediatrics. 2000;105(4):E47.

7. Gall O, Annequin D, Benoit G, Glabeke E, Vrancea F, Murat I. Adverse events of premixed nitrous oxide and oxygen for procedural sedation in children. Lancet. 2001;358(9292):1514-5.

8. Maxton FJ. Parental presence during resuscitation in the PICU: the parents' experience. Sharing and surviving the resuscitation: a phenomenological study. J Clin Nurs. 2008;17(23):3168-76.
9. Gauillard J, Cheref S, Vacherontrystram MN, Martin JC. Chloral hydrate: a hypnotic best forgotten? Encephale. 2002;28(3 Pt 1):200-4.

10. Frankel RI. The Papoose Board and mothers' attitudes following its use. Pediatr Dent. 1991;13(5):284-8.

11. Carr KR, Wilson S, Nimer S, Thornton Jr JB. Behavior management techniques among pediatric dentists practicing in the southeastern United States. Pediatr Dent. 1999;21(6):347-53.

12. Cote CJ, Wilson S. Guidelines for monitoring and management of pediatric patients during and after sedation for diagnostic and therapeutic procedures: an update. Pediatrics. 2006;118(6):2587-602.

13. Hosey MT. UK National Clinical Guidelines in Paediatric Dentistry. Managing anxious children: the use of conscious sedation in paediatric dentistry. Int $\mathrm{J}$ Paediatr Dent. 2002;12(5):359-72.

14. Kupietzky A. Strap him down or knock him out: is conscious sedation with restraint an alternative to general anaesthesia? Br Dent J. 2004;196(3):133-8.

15. Royal College of Nursing. Restraining, holding still and containing children and young people. Guidance for nursing staff. http://www2 ren org uk/cyp/ resources/a-z_of_resources/restraint [2003 cited 2006 Mar. 4].

16. Folkes K. Is restraint a form of abuse? Paediatr Nurs 2005;17(6):41-4.

17. Hardy M, Armitage G. The child's right to consent to $\mathrm{x}$-ray and imaging investigations: issues of restraint and immobilization from a multidisciplinary perspective. J Child Health Care. 2002;6(2):107-19.

18. NCEPOD. Extremes of age. 1999. The national confidential enquiry into perioperative deaths. 
19. Barbi E, Petaros P, Badina L, Pahor T, Giuseppin I, Biasotto E, et al. Deep sedation with propofol for upper gastrointestinal endoscopy in children, administered by specially trained pediatricians: a prospective case series with emphasis on side effects. Endoscopy. 2006;38(4):368-75.

20. Rex DK, Deenadayalu VP, Eid E, Imperiale TF, Walker JA, Sandhu K, et al. Endoscopist-directed administration of propofol: a worldwide safety experience. Gastroenterology 2009;137(4):1229-37; quiz 518-9.

21. Green SM, Krauss B. Barriers to propofol use in emergency medicine. Ann Emerg Med. 2008;52: 392-8.

22. Strunin L. Intravenous conscious sedation for dental treatment: am I my brother's keeper? Anaesthesia. 2007;62(7):645-7.

23. Leroy PL, Blokland Loggers HE, Nieman FHM, Schipper DM. Annual Conference of Dutch Pediatrics. Dutch Journal of Pediatrics, supplement, 2009:117-118.

24. Babl F, Munro FJ, Kainey G, Palmer GM, Iser A. Scope for improvement: hospital wide sedation practice at a children's hospital. Arch Dis Child. 2006;91(8):716-7.

25. Leroy PL, Nieman FH, Blokland-Loggers HE, Schipper DM, Zimmermann LJ, Knape JT. Adherence to safety guidelines on paediatric procedural sedation: the results of a nationwide survey under general paediatricians in The Netherlands. Arch Dis Child. 2010;95(12):1027-30.

26. Yldzdas D, Yapcoglu H, Ylmaz HL. The value of capnography during sedation or sedation/analgesia in pediatric minor procedures. Pediatr Emerg Care. 2004;20(3):162-5.

27. Taylor CJ, Derrick G, McEwan A, Haworth SG, Sury MR. Risk of cardiac catheterization under anaesthesia in children with pulmonary hypertension. $\mathrm{Br} \mathrm{J}$ Anaesth. 2007;98(5):657-61.

28. Royal College of Anaesthetists and Royal College of Radiologists. Sedation and anaesthesia in radiology. Report of a joint working party. 1992. London.

29. Academy of Medical Royal Colleges. Safe sedation practice for healthcare procedures in adults. Report of an intercollegiate working party chaired by the Royal College of Anaesthetists. 2001. Academy of Medical Royal Colleges.

30. SIGN. Safe sedation of children undergoing diagnostic and therapeutic procedures. A national clinical guideline. Scottish Intercollegiate Guidelines Network. 2004. Scottish Intercollegiate Guidelines Network.

31. Levati A, Paccagnella F, Pietrini D, Buscalferri A, Calamandrei M, Grossetti R, et al. SIAARTISARNePI Guidelines for sedation in pediatric neuroradiology. Minerva Anestesiol. 2004;70(10):675-97.

32. Knape JT, Adriaensen H, van Aken H, Blunnie WP, Carlsson C, Dupont M, et al. Guidelines for sedation and/or analgesia by non-anaesthesiology doctors. Eur J Anaesthesiol. 2007;24(7):563-7.
33. Cote CJ. Round and round we go: sedation - what is it, who does it, and have we made things safer for children? Paediatr Anaesth. 2008;18(1):3-8.

34. Department of Health/Faculty of General Dental Practice (UK). Guidleines for the appointment of dentists with a special interest (DwSI) in conscious sedation. 2008. Department of Health UK.

35. Vangerven M, Van Hemelrijck J, Wouters $P$, Vandermeersch E, Van Aken H. Light anaesthesia with propofol for paediatric MRI. Anaesthesia. 1992;47:706-7.

36. Sury M, Harker H, Thomas M. Sedation for MRI using sevoflurane. Paediatr Anaesth. 2005;15(11):1025.

37. De Sanctis Briggs V. Magnetic resonance imaging under sedation in newborns and infants: a study of 640 cases using sevoflurane. Paediatr Anaesth. 2005;15:9-15.

38. Holroyd I. Conscious sedation in pediatric dentistry. A short review of the current UK guidelines and the technique of inhalational sedation with nitrous oxide. Pediatr Anesth. 2008;18:13-7.

39. Motas D, McDermott NB, VanSickle T, Friesen RH. Depth of consciousness and deep sedation attained in children as administered by nonanaesthesiologists in a children's hospital. Paediatr Anaesth. 2004;14(3): 256-60.

40. Krauss B, Green SM. Training and credentialing in procedural sedation and analgesia in children: lessons from the United States model. Paediatr Anaesth. 2008;18(1):30-5.

41. Sury MRJ, Hatch DJ, Deeley T, Dicks-Mireaux C, Chong WK. Development of a nurse-led sedation service for paediatric magnetic resonance imaging. Lancet. 1999;353:1667-71.

42. Keengwe IN, Hegde S, Dearlove O, Wilson B, Yates $\mathrm{RW}$, Sharples A. Structured sedation programme for magnetic resonance imaging examination in children. Anaesthesia. 1999;54(11):1069-72.

43. Woodthorpe C, Trigg A, Alison G, Sury M. Nurse led sedation for paediatric MRI: progress and issues. Paediatr Nurs. 2007;19(2):14-8.

44. Jackson EA, Rabbette PS, Dezateux C, Hatch DJ, Stocks J. The effect of triclofos sodium sedation on respiratory rate, oxygen saturation, and heart rate in infants and young children. Pediatr Pulmonol. 1991;10(1):40-5.

45. Sury MR, Smith JH. Deep sedation and minimal anesthesia. Paediatr Anaesth. 2008;18(1):18-24.

46. Koroglu A, Teksan H, Sagir O, Yucel A, Toprak HI, Ersoy OM. A comparison of the sedative, hemodynamic, and respiratory effects of dexmedetomidine and propofol in children undergoing magnetic resonance imaging (see comment). Anesth Analg. 2006;103(1):63-7.

47. Koroglu A, Demirbilek S, Teksan H, Sagir O, But AK, Ersoy MO. Sedative, haemodynamic and respiratory effects of dexmedetomidine in children undergoing magnetic resonance imaging examination: preliminary results. Br J Anaesth. 2005;94(6):821-4.

48. The Association of Anaesthetists of Great Britain and Ireland. Provision of Anaesthetic Services in Magnetic Resonance Units. 2002. Ref Type: Pamphlet. 
49. Zorab JS. A general anaesthesia service for magnetic resonance imaging. Eur J Anaesthesiol. 1995;12(4): 387-95.

50. Levati A, Colombo N, Arosio EM, Savoia G, Tommasino C, Scialfa G, et al. Propofol anaesthesia in spontaneously breathing paediatric patients during magnetic resonance imaging. Acta Anaesthesiol Scand. 1996;40(5):561-5.

51. Machata AM, Willschke H, Kabon B, Kettner SC, Marhofer P. Propofol-based sedation regimen for infants and children undergoing ambulatory magnetic resonance imaging. Br J Anaesth. 2008;101(2):239-43.

52. Akin A, Esmaoglu A, Guler G, Demircioglu R, Narin N, Boyaci A. Propofol and propofol-ketamine in pediatric patients undergoing cardiac catheterization. Pediatr Cardiol. 2005;26(5):553-7.

53. Rautiainen P, Meretoja OA. Ketamine boluses with continuous low-dose fentanyl for paediatric sedation during diagnostic cardiac catheterization. Paediatr Anaesth. 1993;3:345-51.

54. Donmez A, Kizilkan A, Berksun H, Varan B, Tokel K. One center's experience with remifentanil infusions for pediatric cardiac catheterization. J Cardiothorac Vasc Anesth. 2001;15(6):736-9.

55. Bennett D, Marcus R, Stokes M. Incidents and complications during pediatric cardiac catheterization. Paediatr Anaesth. 2005;15(12):1083-8.

56. Michaud L. Sedation for diagnostic upper gastrointestinal endoscopy: a survey of the Francophone Pediatric Hepatology, Gastroenterology, and Nutrition Group. Endoscopy. 2005;37(2):167-70.

57. Stringer MD, McHugh PJM. Paediatric endoscopy should be carried out under general anaesthesia. $\mathrm{Br}$ Med J. 1995;311:452-3.

58. Lamireau T, Dubreuil M, Daconceicao M. Oxygen saturation during esophagogastroduodenoscopy in children: general anesthesia versus intravenous sedation [see comments]. J Pediatr Gastroenterol Nutr. 1998;27(2):172-5.

59. Barbi E, Gerarduzzi T, Marchetti F, Neri E, Verucci E, Bruno I, et al. Deep sedation with propofol by nonanesthesiologists: a prospective pediatric experience. Arch Pediatr Adolesc Med. 2003;157(11):1097-103.

60. Perera C, Strandvik GF, Malik M, Sen S. Propofol anesthesia is an effective and safe strategy for pediatric endoscopy. Pediatr Anesth. 2006;16(2A):220-1.

61. Culshaw V, Yule M, Lawson R. Considerations for anaesthesia in children with haematological malignancy undergoing short procedures. Paediatr Anaesth. 2003; 13:375-83.

62. Glaisyer HR, Sury MR. Recovery after anesthesia for short pediatric oncology procedures: propofol and remifentanil compared with propofol, nitrous oxide, and sevoflurane. Anesth Analg. 2005;100(4):959-63.

63. McGlone RG, Howes MC, Joshi M. The Lancaster experience of 2.0 to $2.5 \mathrm{mg} / \mathrm{kg}$ intramuscular ketamine for paediatric sedation: 501 cases and analysis. Emerg Med J. 2004;21(3):290-5.

64. Mintegi S, Shavit I, Benito J. Pediatric emergency care in europe: a descriptive survey of 53 tertiary medical centers. Pediatr Emerg Care. 2008;24(6):359-63.
65. Morton NS. Ketamine for procedural sedation and analgesia in pediatric emergency medicine: a UK perspective. Paediatr Anaesth. 2008;18(1):25-9.

66. Shaw AJ, Meechan JG, Kilpatrick NM, Welbury RR. The use of inhalation sedation and local anaesthesia instead of general anaesthesia for extractions and minor oral surgery in children: a prospective study. Int J Paediatr Dent. 1996;6:7-11.

67. Foley J. Nitrous oxide inhalation sedation: what do patients, carers and dentists think about it? Eur J Paediatr Dent. 2005;6(1):23-9.

68. Department of Health U. Conscious sedation in the provision of dental care: report of an Expert Group on Sedation for Dentistry, Standing Dental Advisory Committee. www doh gov uk/dental [2003 cited 2006 Mar. 15]; Available from: URL:www.doh.gov.uk/dental.

69. Department of Health UK - Report by a Group chaired by the Chief Medical Officer and Chief Dental Officer. A Conscious Decision: A review of the use of general anaesthesia and conscious sedation in primary dental care.http://www.dh.gov.uk/en/Publicationsandstatistics/ Publications/PublicationsPolicyAndGuidance/ DH_4074702.2000.

70. Averley PA, Girdler NM, Bond S, Steen N, Steele J. A randomised controlled trial of paediatric conscious sedation for dental treatment using intravenous midazolam combined with inhaled nitrous oxide or nitrous oxide/sevoflurane. Anaesthesia. 2004;59(9):844-52.

71. Klingberg G. Pharmacological approach to the management of dental anxiety in children-comments from a Scandinavian point of view. Int J Paediatr Dent. 2002;12(5):357-8.

72. Wilson KE, Welbury RR, Girdler NM. A study of the effectiveness of oral midazolam sedation for orthodontic extraction of permanent teeth in children: a prospective, randomised, controlled, crossover trial. Br Dent J. 2002;192:457-62.

73. Day PF, Power AM, Hibbert SA, Paterson SA Effectiveness of oral midazolam for paediatric dental care: a retrospective study in two specialist centres. Eur Arch Paediatr Dent. 2006;7(4):228-35.

74. Leelataweedwud P, Vann Jr WF. Adverse events and outcomes of conscious sedation for pediatric patients: study of an oral sedation regimen. J Am Dent Assoc. 2001;132(11):1531-9.

75. Wilson KE, Girdler NM, Welbury RR. Randomized, controlled, cross-over clinical trial comparing intravenous midazolam sedation with nitrous oxide sedation in children undergoing dental extractions. $\mathrm{Br} \mathrm{J}$ Anaesth. 2003;91(6):850-6.

76. Rodrigo MR, Jonsson E. Conscious sedation with propofol. Br Dent J. 1989;166(3):75-80.

77. The Royal College of Anaesthetists and the Faculty of Dental Surgery of the Royal College of Surgeons. Standards for Conscious Sedation in Dentistry: Alternative Techniques. A Report from the Standing Committee on Sedation for Dentistry. http://www. rcoa.ac.uk/docs/SCSDAT.pdf. 2007.

78. Faculty of dental surgery tRCoSoE, The Royal College of Anaesthetists. Standards for conscious sedation in dentistry:alternative techniques. Report 
from the Standing Committee on sedation for dentistry. 2007. London.

79. Mikhael MS, Wray S, Robb ND. Intravenous conscious sedation in children for outpatient dentistry. $\mathrm{Br}$ Dent J. 2007;203(6):323-31.

80. Davies MW, Nind D. Anaesthesia for exodontia in young children - a snapshot survey of European practice. Anaesthesia. 1996;51:713.

81. Worthington LM, Flynn PJ, Strunin L. Death in the dental chair: an avoidable catastrophe ? Br J Anaesth. 1998;80(2):131-2.

82. Sury M, Bullock I, Rabar S, Demott K. Sedation for diagnostic and therapeutic procedures in children and young people: summary of NICE guidelines. BMJ. 2010;341:c6819.

83. National Institute for Health and Clinical Excellence. Sedation for diagnostic and therapeutic procedures in children and young people. (Clinical guideline 112.) 2010. http://guidance.nice.org.uk/CG112.

84. Summary of Draft Guidelines on Sedation and/or Analgesia (PSA) at Locations Outside the Operating Theatre. Part III: In Children. Initiators: Netherlands Society of Anesthesiologists and Dutch Society of Pediatrics. Organization: Dutch Institute for Healthcare Improvement (Central Accompaniment Organization (CBO)). 2011. 



\section{Samenvatting}

\section{Procedurele sedatie en/of analgesie bij kinderen: hoe kan het beter?}

\section{Een zoektocht - vanuit de praktijk, via de evidence - naar praktische oplossingen}

Zieke kinderen moeten regelmatig medische verrichtingen ('procedures') ondergaan die pijnlijk en/of stresserend zijn, of die vereisen dat het kind langdurig stil ligt.

Ondanks het gebruik van lokale pijnstilling, afleidingstechnieken en troosten zullen de meeste (kleine) kinderen zich tegen dergelijke procedures verzetten. Hierdoor kunnen procedures (gedeeltelijk) mislukken, tenzij gebruik wordt gemaakt van dwang of fixatie. Vooral jonge kinderen hebben daarom vaak een vorm van Procedurele Sedatie en/of Analgesie (PSA) nodig.

PSA kan worden gedefinieerd als "het gebruik van sedativa, sederende analgetica of dissociatieve middelen tijdens een diagnostische en/of therapeutische procedure met de bedoeling anxiolyse, pijnstilling, sedatie en/of immobiliteit te bewerkstelligen". De vraag naar kwalitatief hoogstaande PSA bij kinderen wordt steeds groter. Daar bestaan verschillende redenen voor. Ten eerste wordt in de gezondheidszorg voor kinderen in toenemende mate gebruik gemaakt van belastende diagnostische en therapeutische interventies die zonder een vorm van PSA niet comfortabel en/of niet succesvol zijn. Ten tweede wordt er een steeds groter belang gehecht aan een pijn- en stressarme medische zorg voor kinderen. Dit geldt in het bijzonder voor kinderen met een chronische aandoening die vaak herhaaldelijk pijnlijke en/of stresserende procedures moeten ondergaan. Onderzoek heeft aangetoond dat kinderen deze procedures vaak aanduiden als de meest negatieve ervaring van hun ziekte. Hierdoor bestaat een grote kans op anticiperende angst voor vervolgprocedures en nog meer verzet. Patiënten die op kinderleeftijd regelmatig worden geconfronteerd met procedurele pijn en angst zullen later in hun leven vaker noodzakelijke gezondheidszorg mijden. Ten slotte worden in de afgelopen jaren steeds nadrukkelijker ethische en juridische vragen gesteld bij het gebruik van geforceerde dwang en fixatie tijdens niet-levensreddende procedures op de kinderleeftijd.

Ook binnen de Nederlandse (kinder)geneeskunde wordt bij zieke kinderen al decennialang gebruik gemaakt van PSA. In 1998 verscheen zelfs een consensusrichtlijn over dit onderwerp. Ondanks het bestaan van deze richtlijn deden zich in het daarna volgende decennium verschillende ernstige accidenten voor tijdens PSA bij kinderen. Ten minste twee daarvan hadden een dodelijke afloop. Deze accidenten, waarvan vermoed wordt dat ze slechts het topje van een ijsberg zijn, hebben kunnen plaatsvinden omdat onvoldoende aandacht werd besteed aan speci- 
fieke veiligheidsomstandigheden. In opdracht van de Inspectie van de Volksgezondheid, de Nederlandse Vereniging voor Anesthesiologie en de Nederlandse Vereniging voor Kindergeneeskunde heeft een werkgroep met vertegenwoordigers van 21 beroepsverenigingen met ondersteuning van het Kwaliteitsinstituut voor de Gezondheidzorg CBO, een nieuwe richtlijn opgesteld voor PSA. Deze richtlijn, die een vervolg is op de in 1998 uitgebrachte richtlijn, zal vermoedelijk in de loop van 2012 worden geautoriseerd. De dringende noodzaak tot een implementeerbare en praktische richtlijn vormde de belangrijkste aanleiding voor het onderzoek dat in dit proefschrift wordt gepresenteerd. Door middel van dit onderzoek willen we proberen een correcte kwaliteitsanalyse te maken van de actuele PSA praktijk binnen de Nederlandse (algemene) kindergeneeskunde en deze te toetsen aan de beschikbare wetenschappelijke evidence. Op basis van die evidence formuleren we tevens aanbevelingen waarvan de implementatie in de dagelijkse praktijk zal leiden tot het optimaliseren van de kwaliteit van PSA, zowel voor wat betreft de veiligheid als de effectiviteit ervan. Het ultieme doel van dit onderzoek is het op de werkvloer beschikbaar worden van technieken en methodes waarmee de medische professional op een veilige manier medische verrichtingen bij kinderen succesvol en maximaal comfortabel kan maken.

\section{Hoofdstuk 1}

In hoofdstuk 1 plaatsen we in twee aparte delen het onderwerp van dit proefschrift in een historische context.

Het eerste deel van dit hoofdstuk vat de persoonlijke 'tocht' samen die de auteur heeft afgelegd alvorens bij dit proefschrift aan te komen. Deze zoektocht was noodzakelijk om gaandeweg de essentiële onderdelen van een hoogstaande PSA bij kinderen te leren begrijpen. Zeer belangrijk daarbij is de 'ontdekking' van de zogenaamde sedatieparadox. Hiermee wordt een actuele situatie omschreven waarin PSA bij kinderen in de meeste gevallen wordt verricht door professionals (vb kinderartsen) die daar niet specifiek zijn voor opgeleid, terwijl anesthesiologen, de specialisten die wél zijn opgeleid voor het onderdrukken van het bewustzijn tijdens verrichtingen, zich om allerlei redenen zelden bezighouden met PSA. Deze paradox verklaart in belangrijke mate de problemen die bestaan ten aanzien van effectiviteit en veiligheid van PSA bij kinderen.

In het tweede deel vatten we de definities, outcome parameters en internationale historische context van PSA samen. Door de beperkte beschikbaarheid van anesthesiologische ondersteuning hebben niet-anesthesiologen, waaronder kinderartsen, hun eigen PSA protocollen ontwikkeld. In de internationale literatuur werd in het afgelopen decennium veel aandacht besteed aan de slechts matige kwaliteit van deze praktijk. Ten eerste zijn vele van deze protocollen gebaseerd op het gebruik van matig tot slecht titreerbare medicijnen waarvan het precieze effect slechts beperkt voorspelbaar is. Voorbeelden van deze medicijnen zijn chloralhydraat, benzodiazepines, antihistaminica, langwerkende opiaten (morfine, pethidine) en zogenaamde cocktails van verschillende types sederende medicijnen. Ten tweede zijn de professionals die PSA bij kinderen verrichten daar over het algemeen niet voor opgeleid. Ten slotte blijkt dat PSA op geen enkele manier beschouwd wordt als een formele medische handeling en bijgevolg vaak alle transparantie mist. Bovenstaande problemen hebben belangrijke gevolgen voor zowel de veiligheid als de effectiviteit van PSA. 


\section{Er is een probleem met betrekking tot de veiligheid van PSA}

Hoewel de middelen die doorgaans door niet-anesthesiologen voor PSA worden gebruik meestal beschouwd worden als milde sedativa en slechts "roesjes" zouden veroorzaken, kunnen ze in bepaalde, onvoorspelbare omstandigheden aanleiding geven tot ernstige ongewenste neveneffecten die, indien slecht herkend en behandeld, potentieel fataal kunnen zijn voor het kind. De overgrote meerderheid van deze complicaties (>95\%) betreft respiratoire complicaties die het gevolg zijn van het onderdrukken van de ademhaling en/of het verlies van luchtwegcontrole. Deze ernstige neveneffecten, ontstaan meestal door de combinatie van enerzijds een onverwacht dieper en/of langer sedatie effect en anderzijds onvoldoende voorzorgen om ze tijdig te ontdekken en adequaat te behandelen. Uit onderzoek blijkt dat onveiligheid van PSA vooral te maken heeft met gebrekkige randvoorwaarden ten aanzien van patiëntenvoorbereiding, monitoring, professionele competenties en veiligheidsvoorzorgen, dan met de farmacologische kenmerken van het medicijn dat voor PSA wordt gebruikt.

Het rapporteren in de literatuur van ernstige en fatale incidenten heeft internationaal geleid tot het opstellen van richtlijnen voor PSA bij kinderen. Er bestaat tegenwoordig grote internationale eensgezindheid over de veiligheidscriteria waaraan een PSA bij kinderen moet voldoen: (1) een PSA moet worden voorafgegaan door een systematische risicoanalyse en een formele informed consent. (2) Patiënten moeten in principe nuchter zijn en (3) hun vitale parameters moeten adequaat worden bewakt vanaf de toediening van het sedativum totdat ze volledig wakker zijn; (4) Professionals die PSA verrichten moeten daarvoor aantoonbaar competent zijn en (5) in geval van een onverwachte calamiteit moeten alle noodvoorzieningen onmiddellijk adequaat inzetbaar zijn. Onderzoek heeft aangetoond dat het toepassen van deze richtlijnen leidt tot een zeer hoge veiligheid van PSA.

\section{Er is een probleem met betrekking tot de effectiviteit en beschikbaarheid van PSA}

Dagelijks mislukken dure en belastende onderzoeken bij kinderen omdat de toegepaste PSA onvoldoende effectief is. Belangrijkste redenen voor deze ineffectiviteit zijn de beperkte voorspelbare effectiviteit van de gebruikte medicijnen, de afwezigheid van een goed gecoördineerd zorgtraject voor deze PSA's en de beperkte competenties van niet-anesthesiologen om een voorspelbare sedatiediepte te realiseren. Medicijnen met een beduidend betere voorspelbaarheid (bijvoorbeeld Ketamine en Propofol) zijn meestal van oorsprong anesthetica en worden daarom beschouwd als 'alleen veilig in handen van een anesthesioloog'. Toch blijven deze suboptimale vormen van PSA bestaan omdat er meestal geen goed alternatief is als gevolg van een onvoldoende beschikbaarheid van professionals (met name: anesthesiologen) die de competenties hebben om een effectief en titreerbaar sedatieniveau te garanderen.

In het laatste decennium werd in de medische literatuur veel aandacht besteed aan oplossingen voor bovenstaande problemen. Er bestaat een duidelijke trend om PSA toe te vertrouwen aan specifiek opgeleide professionals. Er bestaat overtuigend wetenschappelijk bewijs dat ook niet-anesthesiologen die getraind zijn in PSA bij kinderen een optimale veiligheid en effectiviteit kunnen garanderen. Dit geldt ook voor PSA waarbij gebruik wordt gemaakt van de intraveneuze anesthetica propofol en ketamine. 


\section{Hoofdstuk 2}

In hoofdstuk 2 onderzoeken we de kwaliteit van PSA bij kinderen in de Nederlandse algemene kindergeneeskunde.

In een eerste subhoofdstuk wordt de veiligheid van de gemiddelde PSA praktijk onderzocht. Daarvoor gebruikten we een indirecte techniek waarbij algemeen kinderartsen ondervraagd werden naar de mate waarin ze de bestaande veiligheidsrichtlijnen voor PSA toepassen. Omdat het bestaan van perfect gedrag (i.e. alle handelingen zijn volledig in lijn met de bestaande veiligheidsaanbevelingen) onwaarschijnlijk is en bijgevolg kan verwacht worden dat onperfect gedrag meer de regel dan de uitzondering zal zijn, kozen we er voor om door middel van een individueel rapportpunt (RP; 0-10) de mate van 'afwijking' van perfect handelen te meten. Een RP $\geq 9$ beschouwden we als voldoende om de veiligheid van PSA optimaal te garanderen, daar waar we een RP $<6$ beschouwden als onaanvaardbaar laag. We vonden dat voor de meeste veiligheidsaanbevelingen slechts een kleine minderheid van de kinderartsen een voldoende score haalden, daar waar een substantieel groot aantal van hen een RP van $<6$ scoorden voor essentiële veiligheidsvoorwaarden. Met dit onderzoek konden we aantonen dat potentieel onveilige PSA omstandigheden wijd verspreid zijn binnen de Nederlandse algemene kindergeneeskunde.

In een tweede en derde subhoofdstuk wordt de effectiviteit van de gemiddelde PSA praktijk onderzocht. Hiervoor inventariseerden we eerst de PSA methodes die courant door Nederlandse algemene kinderartsen worden toegepast bij patiënten die een MRI onderzoek moeten ondergaan en bij patiënten die een gastro-intestinale endoscopie moeten ondergaan. Deze onderzoeken werden geselecteerd omdat ze voor algemene kinderartsen de twee belangrijkste aanleidingen zijn voor het voorschrijven van PSA. Daarnaast zochten we voor elk van deze procedures naar een antwoord op de vraag 'welke PSA techniek is op de kinderleeftijd het meest geschikt om deze procedure veilig en effectief te laten slagen'. Om het antwoord te vinden maakten we een systematic review (SR) van de recente wetenschappelijke literatuur. De resultaten van deze SR werden vervolgens vergeleken met de bevindingen uit de analyse van de dagelijkse praktijk. In het algemeen konden we concluderen dat in de algemene kindergeneeskunde nog heel frequent gebruik wordt gemaakt van PSA technieken die aantoonbaar ineffectief of slechts matig effectief zijn. Hierdoor staat vast dat onderzoeken geheel of gedeeltelijk mislukken en/of niet comfortabel voor de patiënt verlopen. Het niet aanwenden van aantoonbaar effectieve methodes (waaronder het gebruik van titreerbare anesthetica zoals propofol) wordt vooral verklaard door het ontbreken van inzetbaarheid van competente professionals.

In een laatste subhoofdstuk gaan we op zoek naar de onderliggende gevoelens (verwachtingen, percepties, evaluaties, angsten/onzekerheden) die bij algemeen kinderartsen bestaan ten aanzien van het verrichten van PSA bij hun patiënten. We onderzochten daarbij in de eerste plaats de mate van (on)tevredenheid (i.e. (dis)satisfaction) met de eigen PSA praktijk. Vervolgens onderzochten we of deze tevredenheid voorspelbaar was vanuit bepaalde onderliggende overtuigingen en/of individuele achtergrondkarakteristieken. Dit onderzoek werd verricht omdat we menen dat het kennen van de variatie en het begrijpen van de mate van (on)tevredenheid interessante en relevante informatie kan genereren die van belang is voor het succesvol implementeren van projecten die bedoeld zijn om de kwaliteit van de PSA-praktijk te verbeteren. We vonden dat de mate van (on)tevredenheid met de eigen PSA-praktijk sterk varieerde tussen professionals onderling. Dat betekent dat een eventueel implementatieplan in een zeer heterogene groep van professionals 'landt': sommigen zijn zeer ontevreden met de eigen PSA praktijk, terwijl anderen juist heel tevreden zijn. Het is waarschijnlijk dat algemene initiatieven voor het 
verbeteren van PSA bij deze twee extremen ook verschillende uitkomsten zullen hebben. Verder vonden we dat de mate van (on)tevredenheid voorspeld kan worden door een aantal onderliggende overtuigingen, meer bepaald door 'de overtuiging dat PSA een relevant probleem is', 'het gevoel dat PSA tot extra werkbelasting leidt', 'zorgen over de (on)veiligheid van de eigen PSA praktijk', bij professionals met minder zelfvertrouwen 'het onvoldoende beroep kunnen doen op anesthesiologische ondersteuning' en bij gebruikers van een PSA protocol 'de overtuiging dat men niet over voldoende materialen of infrastructuur beschikt'. Deze gevoelend verklaren $78.2 \%$ van de totale variatie in (on)tevredenheid. We concludeerden dat een project ter verbetering van de kwaliteit van PSA door algemeen kinderartsen met deze voorspellers van (on)tevredenheid dient rekening te houden.

\section{Hoofdstuk 3}

In hoofdstuk 1 beschreven we de 'sedatieparadox' en de onvoldoende inzetbaarheid van anesthesiologen om alle verzoeken tot PSA te kunnen uitvoeren. De inzet van niet-anesthesiologen voor PSA bij kinderen is daarom onvermijdbaar. Recent onderzoek toont aan dat adequaat opgeleide professionals in staat zijn om effectieve en veilige PSA bij kinderen te bieden, inclusief het gebruik van krachtige sedativa/anesthetica zoals propofol, ketamine en kortwerkende opiaten.

In hoofdstuk 3 onderzochten we door middel van een systematic review van de recente literatuur welke 'skills' en 'competenties' een PSA-professional dient te bezitten om een hoogkwalitatieve PSA service te kunnen bieden. Bij gebrek aan prospectief vergelijkend onderzoek tussen verschillende competentieniveaus, pasten we een indirecte methode toe waarbij we de resultaten afleidden uit rapporten over veiligheid en effectiviteit van verschillende soorten PSA voor uiteenlopende procedures bij kinderen.

Hoewel de veiligheidsprofielen van de verschillende PSA medicijnen zeker verschillend zijn, worden de noodzakelijke skills en competenties die nodig zijn om tijdig bijwerkingen te diagnosticeren én adequaat te behandelen in het algemeen bepaald door de waarschijnlijkheid dat zich ernstige incidenten kunnen voordoen en door de mate van voorspelbaarheid van duur en diepte van sedatie in het bijzonder. Het ligt daarom voor de hand om aparte competenties te definiëren voor enerzijds matige tot diepe sedatie en anderzijds lichte sedatie.

De mate van effectiviteit wordt hoofdzakelijk bepaald door de volgende competenties: het kunnen gebruiken van kortwerkende, titreerbare medicijnen ( $\mathrm{vb}$ kortwerkende anesthetica en/of opiaten voor invasieve procedures; lachgas mengsels voor milde procedures), het kunnen toepassen van topicale anesthesietechnieken en het kunnen toepassen van non-farmacologische technieken.

In hoofdstuk 3 hebben we tevens de conclusies uit de literatuur vertaald tot aanbevelingen. Deze kunnen de basis vormen van de inhoud van opleiding in PSA en van de eindtermen waaraan een PSA-professional moet voldoen. Zie ook de tabellen 4, 5a en $5 \mathrm{~b}$ in hoofdstuk 3.

\section{Hoofdstuk 4}

Tijdens een pijnlijke en/of medische verrichting zullen kinderen zich niet zelden verzetten. Als hierdoor de verrichting dreigt te mislukken, kan overwogen worden om het kind fysisch te dwin- 
gen de procedure te ondergaan. Hoewel deze toepassing van dwang tot de dagelijkse kindergeneeskundige zorg hoort, stuit ze tegen belangrijke bezwaren.

In dit hoofdstuk beschrijven we deze bezwaren en vatten we de bestaande literatuur en richtlijnen over dit onderwerp samen. Tevens wordt dwangtoepassing getoetst aan gezondheidsethische en -juridische overwegingen. Het wel of niet toepassen van fysische dwang dient te worden benaderd vanuit het oogpunt van kwaliteit van zorg. ledereen, ook een kind, heeft recht op goede zorg. Tenzij er een noodzaak tot levensreddend handelen bestaat, moet de hulpverlener bij verzet van een kind eerst alle mogelijke alternatieven overwegen en vervolgens kiezen voor de zorg die in het gegeven geval de beste is. Dwangtoepassing kan alleen indien vaststaat dat ze de best mogelijke vorm van zorg is.

\section{Hoofdstuk 5}

In hoofdstuk 5 vatten we de nieuwe evidence-based richtlijn samen die we tussen 2006 en 2009 samen met vertegenwoordigers van 21 wetenschappelijke organisaties en begeleid door experten van het $\mathrm{CBO}$ kwaliteitsinstituut voor de geneeskunde hebben opgesteld. Het initiatief voor deze nieuwe richtlijn, die de oude richtlijn van 1998 moet vervangen, werd genomen door de Nederlandse Vereniging voor Kindergeneeskunde (NVK) en de Nederlandse Vereniging voor Anesthesiologie (NVA), nadat de Inspectie voor de Gezondheidszorg (IGZ) had aangedrongen op een nieuwe en implementeerbare richtlijn. De richtlijntekst is inhoudelijk goedgekeurd door de leden en bestuur van alle participerende verenigingen en heeft dus een groot draagvlak onder de betrokken professionals. Naar verwachting zal deze richtlijn begin 2012 worden geautoriseerd door de initiatiefnemende verenigingen.

De richtlijn is tot stand gekomen volgens de methode van Evidence Based Richtlijn Ontwikkeling (EBRO) wat wil zeggen dat de bestaande wetenschappelijke evidence is gebruikt om conclusies te trekken, die samen met overige overwegingen uit de praktijk de basis vormden voor aanbevelingen voor de praktijk. Het geheel der aanbevelingen beschrijft de voorwaarden voor maximaal veilige en maximaal effectieve PSA bij kinderen. Deze aanbevelingen kunnen worden onderverdeeld in een aantal algemene basisprincipes en in specifieke praktische aanbevelingen ten aanzien van de noodzakelijke randvoorwaarden. Vervolgens worden in een apart deel procedure specifieke aanbevelingen geformuleerd.

\section{ALGEMENE BASISPRINCIPES VAN DE NIEUWE RICHTLIJN}

\section{PSA moet worden beschouwd als een onafhankelijke medische handeling}

Dit impliceert dat alleen aantoonbaar competente professionals op een effectieve en veilige manier een PSA kunnen verrichten. Er zal daarom werk moeten worden gemaakt van een specifiek opleidingstraject en een systeem van (re)certificatie.

PSA is optimaal veilig en effectief indien voldaan wordt aan de noodzakelijke randvoorwaarden en competenties, zoals beschreven in de richtlijn. Het is daarbij niet van belang of de PSA wordt verricht door een anesthesioloog of door een in PSA-competent nietanesthesioloog. 
De belangrijkste randvoorwaarden worden verder beschreven onder de vorm van een aantal praktische aanbevelingen. Wat de noodzakelijke professionele competenties betreft, bestaat er goed wetenschappelijk bewijs dat ook niet-anesthesiologen die getraind zijn in PSA bij kinderen een optimale veiligheid en effectiviteit kunnen garanderen. Dit geldt ook voor PSA waarbij gebruik wordt gemaakt van de intraveneuze anesthetica propofol en ketamine. Op basis van de literatuur kunnen de noodzakelijke competenties worden geïdentificeerd (zie Hoofdstuk 3)

\section{Sedatie en Analgesie zijn verschillende begrippen}

Het is principieel onjuist om pijn te bestrijden met uitsluitend een anxiolyticum of sedativum. Bij pijnlijke procedures moet daarom altijd gebruik worden gemaakt van een lokaal en/of systemisch werkend pijnstillend middel. Het is ontoelaatbaar om voor pijnlijke procedures uitsluitend sedatie te gebruiken, zelfs al hoopt men op een amnesie effect van het middel.

\section{Er moet steeds worden gekozen voor de meest effectieve PSA techniek}

De aard van de uit te voeren procedure, de individuele sedatiebehoefte van de patiënt en de gezondheidstoestand van de patiënt bepalen welk sedatieniveau en welke PSA techniek de meest geëigende is om de ingreep zo succesvol mogelijk te maken op een voor de individuele patiënt zo comfortabel mogelijke manier. Optimaal succesvol betekent dat de PSA techniek streeft naar een 100\% voorspelbaar procedureel succes en timing, een hoge voorspelbaarheid van sedatieniveau en -duur en minimale inductie- en recoverytijden. Optimaal comfortabel betekent minimale tot afwezige pijn en angst voor de patiënt en het ontbreken van een noodzaak tot geforceerde dwang of fixatie (restraint).

De nieuwe richtlijn stelt dat bij niet-levensreddende handelingen in principe geen gebruik mag worden gemaakt van geforceerde dwang en fixatie, tenzij vaststaat dat dit de beste manier is om de hoogste kwaliteit van zorg te waarborgen. Van zodra (een combinatie van) afleidingstechnieken, lokale/topicale anesthesie en lichte sedatie niet volstaan moet gekozen worden voor een effectievere vorm van PSA. Bij (zeer) pijnlijke en/of (zeer) stresserende procedures en bij procedures die langdurige immobiliteit of coöperatie vereisen zal dan meestal diepe sedatie noodzakelijk zijn.

\section{PSA is slechts een onderdeel van een totaal beleid voor procedureel comfort en succes}

Voor procedureel comfort en succes is meestal meer nodig dan alleen maar het kunnen toepassen van een effectieve PSA techniek. De belangrijkste elementen van een totaal beleid voor procedureel succes en comfort worden samengevat in tabel 1 van hoofdstuk 5 . Er bestaat steeds meer bewijs voor de toepassing van non-farmacologische technieken. Voorbereiding door middel van simulatie blijkt bijvoorbeeld een effectieve techniek bij MRI scanning van kinderen. Voor pijnlijke en/of stresserende procedures bij oudere kinderen ( $>4$ jaar) verdient het aanbeveling om systematisch gebruik te maken van afleidingstechnieken of hypnose.

\section{Het sedatiecontinuüm: een glijdende schaal}

In de recente literatuur worden verschillende sedatieniveaus onderscheiden. (Zie Tabel $\mathbf{2}$ in Hoofdstuk 5) De definities mogen niet de indruk wekken dat de sedatieniveaus trapsgewijs 
onderscheidbaar zijn, of individueel voorspelbaar zijn. In de meeste gevallen is veeleer sprake van een glijdend sedatie continuüm waarbij de patiënt onverwacht een onbedoeld dieper sedatieniveau kan bereiken.

De noodzakelijke randvoorwaarden en competenties worden bepaald door het bedoeld sedatieniveau, de toegepaste sedatie techniek, de aard van de uit te voeren procedure en de individuele patiëntenkenmerken en gezondheidstoestand.

Voor wat betreft PSA bij kinderen blijkt uit de literatuur dat matige en diepe sedatie wat betreft voorspelbaarheid en controleerbaarheid niet goed van elkaar te onderscheiden zijn. Kinderen kunnen daardoor onverwacht in een veel dieper dan bedoeld sedatieniveau terechtkomen. Deze conditie blijkt duidelijke geassocieerd te zijn met een verhoogde kans op complicaties. Bovendien bestaat bij kinderen een grote kans dat een bedoelde matige sedatie onvoldoende is en de PSA daarom moet worden 'opgeschaald' naar diepe sedatie. Daarom geldt dat voor matige en diepe sedatie bij kinderen dezelfde randvoorwaarden en competenties noodzakelijk zijn. Voor een PSA met chloralhydraat tijdens een MRI scan of met midazolam plus pethidine tijdens een gastroscopie gelden dus in wezen dezelfde strenge voorwaarden als tijdens een PSA waarbij propofol wordt gebruikt!

Sommige patiënt- of procedure gebonden omstandigheden verhogen de kans op onveilige en/of ineffectieve PSA. In dergelijke situaties wordt best eerst overlegd met een anesthesioloog.

\section{RANDVOORWAARDEN: PRAKTISCHE AANBEVELINGEN}

\section{Informed Consent}

PSA moet beschouwd worden als een aparte medische handeling en niet als een onderdeel van de procedure waarvoor ze wordt verricht. Volgens de regelgeving ten aanzien van de Geneeskundige Behandel Overeenkomst (afdeling 7.7.5 van het Burgerlijk wetboek) dient de patiënt te worden voorgelicht over alle procedures die hij of zij ondergaan. Daarnaast heeft de patiënt of diens vertegenwoordiger het recht om te weten wat de winstverwachting is, welke de kans op complicaties is en of er alternatieven voorhanden zijn. Dat geldt evenzeer voor de PSA. De patient/wettelijk vertegenwoordiger dient dus mondeling geïnformeerd te worden over doel, aard en gevolgen van PSA evenals over risico's en alternatieven voor de voorgestelde pijnstilling/sedatie/anesthesie/restraint) De professional die verantwoordelijk is voor de PSA verschaft de noodzakelijke informatie aan kind/wettelijk vertegenwoordiger en verwerft ook de toestemming van kind/wettelijk vertegenwoordiger. Het is tevens aan te bevelen om in het patiëntendossier vast te leggen voor welke vorm van PSA toestemming is gegeven, door wie (kind en/of wettelijk vertegenwoordiger) toestemming is verleend, aan wie toestemming is verleend, en wanneer dat heeft plaatsgevonden.

\section{Patiënten selectie en risico inschatting}

Patiënten met vooraf bestaande aandoeningen van de ademhaling, circulatie of bewustzijn hebben een hoger risico om onder sedatie potentieel gevaarlijke complicaties te ontwikkelen. Een zorgvuldige risico inschatting is daarom essentieel. De meeste richtlijnen adviseren om 
hiervoor gebruik te maken van de zogenaamde physical status classification van de American Society of Anesthesiologists (ASA) (Zie Tabel 3 in Hoofdstuk 5). Over het algemeen wordt aanvaard dat patiënten met een ASA classificatie van I of II in aanmerking komen voor PSA door een niet-anesthesioloog. De ASA systematiek houdt echter weinig rekening met andere factoren die bij een PSA de kans op complicatie en/of ineffectiviteit verhogen. Voor voorbeelden zie Tabellen 4 en $\mathbf{5}$ in Hoofdstuk 5). Indien een van deze risicofactoren aanwezig is, verdient het de voorkeur om eerst een anesthesioloog te consulteren én de procedure alleen onder PSA te laten doorgaan indien deze verricht kan worden door een (kinder)anesthesioloog of door een specifiek voor PSA getraind professional én nadat indicaties en risico's zorgvuldig werden afgewogen.

\section{Nuchter zijn}

De meeste richtlijnen voor PSA hanteren dezelfde aanbevelingen ten aanzien van nuchterheid als diegene die gelden bij preoperatieve patiënten. $\mathrm{Er}$ is in de literatuur echter geen bewijs te vinden dat het nuchter houden van een patiënt het risico van aspiratie rondom een PSA daadwerkelijk kleiner maakt of dat het niet-nuchter zijn de kans op aspiratie doet toenemen. Daarenboven blijken nuchtere kinderen vaak moeilijker te sederen. Voor lichte sedatie geldt daarom dat kinderen niet nuchter hoeven te zijn. Tijdens matige tot diepe sedatie kan de maaglediging vertraagd zijn of de kans op reflux verhoogd zijn of aspiratie worden uitgelokt door balloneren bij hypoventilatie. Een kind dient daarom bij voorkeur nuchter te zijn voor procedures waarbij matige tot diepe sedatie wordt toegepast. Nochtans is de acute, niet-nuchtere conditie van een kind op zich geen absolute contra-indicatie voor een PSA. Dit is belangrijk indien uitstel van de procedure geassocieerd is met gezondheidsrisico's en/of belangrijk discomfort. Wel zal altijd een nauwkeurige afweging van de risico's op aspiratie moeten plaatsvinden waarbij de keuze van het sedativum, aanpassing van de diepte van sedatie en eventuele bescherming van de luchtweg onderdeel dienen te zijn van deze afweging.

Jonge kinderen worden voor radiologische onderzoeken wel een keer 'gesedeerd' met de combinatie van preprocedurele voeding en inbakeren. Hoewel deze methode vaak succesvol wordt toegepast bij neonaten en jonge zuigelingen, bestaat er geen gepubliceerd onderzoek naar de effectiviteit en veiligheid. Het is wel sterk af te raden om deze methode te combineren met het toedienen van sedativa.

\section{Monitoring}

Het is goed bekend dat de PSA medicijnen kunnen interfereren met de vitale reflexen en functies. Hierdoor kunnen zich onverwacht potentieel ernstige incidenten kunnen voordoen. Meestal gaat het om acuut respiratoir falen en/of hypoxie als gevolg van een ademdepressie en/of luchtwegobstructie. Hypotensie, bradycardie en hartstilstand zijn zeer zeldzaam en eigenlijk altijd het gevolg van een niet tijdig herkend respiratoir probleem. Adequate monitoring is dan ook essentieel om dramatische complicaties te voorkomen. Met monitoring wordt best gestart vóór de toediening van de sedativa zodat men geïnformeerd is over de uitgangsconditie van de patiënt. Vervolgens moet ze ononderbroken doorgaan tot aan het einde van de recovery. Dit betekent dus dat monitoring niet beperkt mag zijn tot de eigenlijke procedure maar moet doorgaan tot de patiënt volledig wakker is.

Voor wat betreft noodzakelijke monitoring moet een onderscheid worden gemaakt tussen enerzijds lichte sedatie en anderzijds matige tot diepe sedatie. Algemeen wordt aanvaard dat 
tijdens lichte sedatie pulse oximetrie en continue verbale interactie met de patiënt volstaan als bewaking. Bij matige en diepe sedatie moeten ook de ademhaling, de luchtweg, het hartritme, de hemodynamiek en het bewustzijn worden bewaakt. Deze aanbevelingen zijn meer gebaseerd op "gezond verstand" dan op prospectief vergelijkend onderzoek tussen verschillende niveaus van monitoring. Vooral de zinvolheid van bloeddrukmeting bij ASA I en II patiënten kan in vraag worden gesteld. Het is bekend dat het opblazen van een bloeddruk cuff tijdens een matige sedatie de patiënt kan doen ontwaken. Wanneer voor PSA gebruik wordt gemaakt van medicijnen waarvan bekend is dat ze de bloeddruk rechtsreeks beïnvloeden, dan lijkt het redelijk om de bloeddruk regelmatig te meten. Nochtans is het onwaarschijnlijk dat PSA geïnduceerde hypertensie ( $\mathrm{vb}$ bij ketamine) of hypotensie ( $\mathrm{vb}$ propofol) een relevant probleem betekenen voor ASA I of II patiënten.

In de nieuwe richtlijn wordt nadrukkelijk gepleit voor het introduceren van capnografie als standaard bewaking van ademhaling en luchtweg tijdens matige en diepe sedatie. Capnografie berust op de non-invasieve meting van de partiële druk van $\mathrm{CO}_{2}$ in de uitgeademde lucht. Deze $\mathrm{CO} 2$ monitor toont zowel een end-tidal pCO2 getal (capnometrie) dat goed overeenstemt met de pCO2 in bloed, als een grafische weergave van de uitademing (Capnografie). Hierdoor ontstaat continue breath-to-breath informatie over de ademhaling en de mate waarin de luchtweg open is. Capnografie behoort tot de standaard monitoring van geïntubeerde patiënten onder anesthesie. Dankzij nieuwe technologie (oa microstream ${ }^{\circledR}$ ) en aangepast meetdevices (speciaal ontworpen neusbrilletjes) kan nu ook capnografie betrouwbaar worden verricht bij nietgeïntubeerde kinderen. Er bestaan ook neusbrilletjes waarmee tegelijk capnografie kan worden verricht én zuurstof kan worden toegediend. (Figuur 1 in deel 1.2 van hoofdstuk 1) Onderzoek heeft aangetoond dat capnografie tijdens PSA het dreigend falen van de ventilatie en/of luchtweg significant sneller detecteert dan klinische observatie of pulse oximetrie. Het gebruik van deze techniek is in het bijzonder geïndiceerd bij elke vorm van diepe sedatie en tijdens PSA voor procedures waarbij de patiënt beperkt of niet direct geobserveerd kan worden (MRI scan, duistere ruimte, afgedekt gelaat).

Het is goed bekend dat de mate van sedatie correleert met de kans op complicaties. Het bewaken van de mate van bewustzijnsdaling biedt bijgevolg theoretische voordelen ten aanzien van de PSA gerelateerde veiligheid. Voor diepe sedatie wordt algemeen aangeraden om het bewustzijn regelmatig te 'meten' zolang er een sederend effect kan bestaan van de toegediende medicatie. De beste manier om het bewustzijn te objectiveren is het gebruik van een gevalideerde observatie instrument zoals de University of Michigan Sedation Scale (UMSS). Er bestaat vooralsnog geen evidence voor het gebruik van technische neuro-monitiring (vb BIS monitoring) als bewaking van het bewustzijn bij PSA.

\section{Recovery en ontslag}

Onmiddellijk na de procedure zijn de toegediende sedativa meestal nog niet uitgewerkt terwijl de pijn- of stressprikkels wel wegvallen. Het is daarom zeer belangrijk dat de hierboven beschreven bewaking doorgaat tot de patiënt volledig wakker is. Tevens verdient het de voorkeur om strikte afspraken te maken over wat beschouwd wordt als "volledig wakker". Langwerkende sedativa (vb chloralhydraat) kunnen tot 24 uur na toediening belangrijke neveneffecten effecten hebben (gedragsveranderingen, braken, evenwichtsstoornissen). Ouders en/of patiënt moeten bij ontslag goed geïnformeerd en geïnstrueerd worden over laattijdige effecten. 


\section{Noodvoorzieningen}

Zeer ernstige complicaties bij PSA komen zelden voor. Nochtans kunnen ademdepressie en luchtwegobstructie onverwacht optreden waarna zeer snel een levensbedreigende situatie kan ontstaan. Tijdens een PSA en de recoveryperiode die erop volgt moet een professional aanwezig zijn die noodsituaties tijdig kan ontdekken en onmiddellijk adequaat kan behandelen.

\section{PROCEDURE SPECIFIEKE AANBEVELINGEN}

\section{Deze worden samengevat in tabel 1.}

Tabel 1: Procedures waarvoor PSA noodzakelijk kan zijn

\begin{tabular}{|c|c|c|c|c|c|}
\hline Type Procedure & Voorbeelden & Type patiënt & $\begin{array}{l}\text { Sedatie- } \\
\text { niveau* }\end{array}$ & $\begin{array}{l}\text { Sedatie- } \\
\text { techniek** }\end{array}$ & $\begin{array}{l}\text { Additionele } \\
\text { technieken }\end{array}$ \\
\hline $\begin{array}{l}\text { Niet-pijnlijke } \\
\text { procedures } \\
\text { waarbij lang- } \\
\text { durig stilliggen } \\
\text { noodzakelijk is. }\end{array}$ & $\begin{array}{l}\text { Beeldvorming } \\
\text { Radiotherapie }\end{array}$ & $\begin{array}{l}\text { Jonge leeftijd } \\
\text { (<6 jaar) } \\
\text { Retardatie } \\
\text { Angstige per- } \\
\text { soonlijkheid }\end{array}$ & $\begin{array}{l}\text { Diepe } \\
\text { sedatie }\end{array}$ & $\begin{array}{l}\text { Titreerbare, } \\
\text { intraveneuze } \\
\text { sedatie met } \\
\text { kortwerkende } \\
\text { medicijnen }\end{array}$ & $\begin{array}{l}\text { Voorbereiding } \\
\text { Afleiding }\end{array}$ \\
\hline $\begin{array}{l}\text { Invasieve en/of } \\
\text { langdurige } \\
\text { procedures die } \\
\text { zeer pijnlijk } \\
\text { en/of zeer } \\
\text { stresserend } \\
\text { kunnen zijn }\end{array}$ & $\begin{array}{l}\text { Beenmergpunctie } \\
\text { Bot-, lever-, nierbiopsie } \\
\text { Reductie fractuur/luxatie } \\
\text { Wondzorg (groot) } \\
\text { Endoscopisch onderzoek } \\
\text { Thoraxdrainage } \\
\text { Centraal veneuze lijn }\end{array}$ & Alle leeftijden & $\begin{array}{l}\text { Diepe } \\
\text { sedatie }\end{array}$ & $\begin{array}{l}\text { Titreerbare, } \\
\text { intraveneuze } \\
\text { analgesie en } \\
\text { sedatie met } \\
\text { kortwerkende } \\
\text { medicijnen }\end{array}$ & $\begin{array}{l}\text { Voorbereiding } \\
\text { Afleiding } \\
\text { Lokale/Topicale } \\
\text { anesthesie }\end{array}$ \\
\hline $\begin{array}{l}\text { Beperkt inva- } \\
\text { sieve procedu- } \\
\text { res die pijnlijk } \\
\text { en/of stresse- } \\
\text { rend kunnen } \\
\text { zijn }\end{array}$ & $\begin{array}{l}\text { Lumbaal punctie } \\
\text { Perifeer Infuus } \\
\text { Bloedafname } \\
\text { Blaascatheterisatie } \\
\text { Maagsonde } \\
\text { Aanprikken Port-a-Cath }{ }^{\circledast} \\
\text { Blaaspunctie } \\
\text { Huidbiopsie } \\
\text { Thoraxpunctie } \\
\text { Hechten snijwond } \\
\text { Wondzorg (klein) }\end{array}$ & $\begin{array}{l}\text { Jonge leeftijd } \\
(<6 \text { jaar) } \\
\text { Retardatie } \\
\text { Angstige per- } \\
\text { soonlijkheid }\end{array}$ & $\begin{array}{l}\text { Lichte } \\
\text { sedatie }\end{array}$ & $\begin{array}{l}\text { Lachgas/O2 } \\
\text { Midazolam }\end{array}$ & $\begin{array}{l}\text { Voorbereiding } \\
\text { Afleiding } \\
\text { Hypnose } \\
\text { Lokale/Topicale } \\
\text { anesthesie }\end{array}$ \\
\hline
\end{tabular}

\footnotetext{
* Bedoeld wordt het sedatieniveau dat in de meerderheid der gevallen wenselijk is. In individuele gevallen kunnen andere sedatiebehoeften bestaan; Daarbij is het wel noodzakelijk dat (1) er een volledige informed consent is van de patiënt (en/of ouders/verzorgers) en de patiënt (en/of ouders/verzorgers) de voorkeur geeft/geven aan deze manier van werken en (2) er tijdens de procedure geen gebruik dient te worden gemaakt van geforceerde dwang en fixatie (restraint).

$* *$ is de sedatietechniek waarvan de hoogste effectiviteit kan worden verwacht. Indien andere vormen van PSA worden gekozen, dan zal de effectiviteit op een of meerdere domeinen van de procedure meestal suboptimaal zijn.
} 


\section{Hoofdstuk 6}

In dit laatste hoofdstuk vatten we de belangrijkste conclusies samen van dit proefschrift en kijken we eveneens naar de toekomst.

Het onderzoek dat we hebben verricht maakt in de eerste plaats duidelijk dat PSA in de Nederlandse algemene kindergeneeskunde een aantal belangrijke problemen kent: de veiligheid is ondermaats, er wordt nog vaak gebruik gemaakt van matig-effectieve technieken (waardoor medische verrichtingen onnodig falen en/of oncomfortabel zijn) en er kan onvoldoende beroep gedaan worden op anesthesiologische ondersteuning of op specifieke PSA expertise. Hoewel we ons in ons onderzoek gefocust hebben op de algemene kindergeneeskunde is het zeer aannemelijk dat gelijkaardige problemen bestaan in andere kindergeneeskundige settings (vb kindergeneeskundige subspecialismen, kinderchirurgie, spoedeisende hulp, beeldvorming, dagkliniek,...). Er bestaan verschillende dwingende en dringende redenen om PSA bij kinderen in de Nederlandse gezondheidszorg te verbeteren. De onvoldoende veiligheid en de matige effectiviteit van courant toegepaste PSA methodes vormen de meest voor hand liggende. Daarnaast bestaan ook ethische en juridische argumenten om procedureel comfort bij kinderen te verbeteren. Het bestaan van effectieve technieken die procedurele angst en pijn kunnen voorkomen of onderdrukken, maakt dat het toepassen van geforceerde immobilisatie en dwang (restraint) in principe in de meeste gevallen niet nodig hoeft te zijn.

In dit proefschrift gaan we ook actief op zoek naar oplossingen. Een brede en solide basis voor toekomstige oplossingen wordt gevormd door de nieuwe evidence based richtlijn (zie hoofdstuk 5). Op basis van onderzoek in andere westerse landen kunnen we aannemen dat het implementeren van deze richtlijn zal leiden tot maximaal veilige en maximaal effectieve PSA voor kinderen. Het realiseren van maximale veiligheid zal vooral afhangen van het verbeteren van de randvoorwaarden (patiëntenselectie, voorbereiding, monitoring, bewaakte recovery en noodvoorzieningen) en het toevertrouwen van PSA aan uitsluitend competente professionals. Voor het optimaliseren van de effectiviteit is het van belang dat beroep kan worden gedaan op krachtige, kortwerkende en titreerbare medicijnen (vb propofol, snelwerkende opiaten) bij invasieve procedures en van adequate technieken voor lichte sedatie (lachgasmengsels, transmucosale midazolam). De weerstanden die momenteel bestaan tegen het gebruik van propofol door niet-anesthesiologen en tegen het toepassen van lachgasmengsels buiten de operatiekamer kunnen op basis van de beschikbare evidence eenvoudig worden ontkracht. Tenslotte zijn preventie, non-farmacologische technieken, lokale/topicale anesthesie en de beschikbaarheid van rescue-anesthesie belangrijke onderdelen van een totaal beleid gericht op procedureel succes en comfort.

Op het einde van hoofdstuk 6 gaan we in op de grootste uitdaging die nu voor ons ligt: de implementatie van de wetenschappelijke evidence in de dagelijkse praktijk. Een combinatie van strategieën is daarbij van belang, waaronder het instellen van een landelijke begeleidingscommissie, een lokale ziekenhuis-sedatiecommissie en het organiseren van opleiding. Dit laatste moet leiden tot het beschikbaar worden van PSA-praktijkspecialisten die de competenties hebben om PSA te verrichten in lijn met de aanbevelingen van de richtlijn. Tenslotte dient een financieel en logistiek kader te worden uitgewerkt waarbinnen optimaal veilige en effectieve PSA mogelijk wordt . 


\section{Epiloog en Dankwoord}

Bij het schrijven van deze zinnen realiseer ik me plots dat het de laatste zijn die ik aan dit proefschrift toevoeg. Alles wat ik als 'wetenschapper' wilde zeggen heb ik hiervóór gezegd. Dus waarom nog verder schrijven? Wil de lezer dit nog wel? Wat doe ik hem/haar aan om na de zware maaltijd nu ook nog een dessert te willen serveren? Of ligt het anders? Vermoedelijk wel! Zijn niet de 'stellingen' (en dan in het bijzonder de 'niet-wetenschappelijke'), het 'CV' ('hoe oud is die kerel eigenlijk?') en het 'dankwoord' ('vergat hij niet mij te bedanken?') de amuses van elk proefschrift? Terwijl de 'plat de resistance' vaak niet eens genuttigd wordt en meestal ongeconsumeerd en onverteerd gedoemd is tot een verticaal leven op een of ander stoffig schap, wachtend op de papierversnipperaar of het edelmoedig transfer naar een derde wereld bibliotheek? Het zou zomaar kunnen. Geen tijd dus voor ijdelheid. De wereld is, ook na dit proefschrift, niet veranderd. Meer nog, al haar zinnen zullen ooit worden achterhaald door nieuwe inzichten en kennis. Ik maak mezelf niks wijs. Hoe "waar" ze nu ook lijken, het is hun lot - Karl Popper indachtig - om ooit weer te worden ontkend, aangepast of aangevuld. Het is voorspelbaar dat dit boek(je), net als alle andere ernstige schrijfsels, ooit als ouderwets of voorbijgestreefd zal worden aangevoeld, of gewoon zal worden vergeten. Straks blijkt dat de inhoud amper solider was dan een zuchtje mist boven een vulkaan. Als schrijver kan je hooguit hopen dat tussen de regels door alle noeste arbeid die eraan vooraf is gegaan nog enige tijd proefbaar blijft. Als een stuwende golfslag die je heel even meevoert door de stroming van het alledaagse, of een lange trage aai over de ijdele bol... Wellicht evenzeer een illusie. Vergankelijkheid en nihilisme zijn me niet echt vreemd. Zolang maar achteraf de inhoud niet één onhaalbaar ideaal zou blijken te zijn. Dat zou pas een reden tot grote treurnis zijn.

Niet zozeer dit ij(de)le boekje, maar vooral de weg die ernaar heeft geleid is vandaag de echte oogst. Samen met vele anderen zijn we er, meen ik, in geslaagd om het delicate en moeilijke thema van procedurele sedatie en/of analgesie (PSA) bij kinderen op de agenda van de Nederlandse (kinder)geneeskunde te plaatsen. Het werk is zeker nog niet klaar, maar er is vooral geen weg terug meer! Deze eerste vruchten kunnen niet anders dan nu verder groeien en zich voortplanten. Dat is in belangrijke mate te danken aan het vele werk dat werd verricht door de leden van de richtlijnenwerkgroep die ik mocht voorzitten. Het schrijven van een evidence-based richtlijn bleek geen sinecure te zijn. Nochtans mogen we trots zijn op het resultaat. Mijn dank voor hen (hun namen heb ik opgelijst in hoofdstuk 5) is dan ook zeer groot. Heel in het bijzonder dank ik Heleen Blokland-Loggers (kinderarts en voormalig voorzitter van de sectie algemene kindergeneeskunde van de Nederlandse Vereniging voor Kindergeneeskunde), Margreet van Bergen (voormalig directeur van de vereniging Kind en Ziekenhuis) en Daphne Schipper (voorma- 
lig adviseur bij het (BO). Deze drie dames, die ook lid waren van de richtlijnenwerkgroep, zijn elk op hun manier voor mij, voor het aangaan van het publieke debat over PSA en voor het realiseren van dit proefschrift een uiterst belangrijke bron van inspiratie geweest. Ook dank ik het bestuur van de Nederlandse Vereniging voor Kindergeneeskunde voor het vertrouwen dat ze in me stelden. Ingrid Maas vervulde daarbij uitstekend ondersteunend werk.

Zoals ik al in het inleidende hoofdstuk schreef: promoveren lag - echt waar - niet in de lijn van mijn bedoelingen. Het kwam veeleer onverwacht om de hoek kijken en het heeft een poos geduurd alvorens ik me liet verleiden. Ik geloof ook nu nog steeds niet dat een doctorstitel van een arts een betere dokter maakt. Bovendien was ik aanvankelijk doodsbenauwd dat het promotiewerk me zou afleiden van het echte medische werk of dat de al hoge werklast van een baan op een kinderlC er ondraaglijk door zou worden. Achteraf bekeken viel het mee, al was de dreiging nooit ver weg.

Anderzijds - en dat beken ik graag en gretig - heeft het promoveren me heel veel gegeven. In de eerste plaats heb ik zeer boeiende en inspirerende mensen leren kennen wiens dagelijks biotoop anders was dan het mijne. Daarnaast heb ik mogen leren hoe krachtig wetenschappelijke methodes kunnen zijn om maatschappelijke en klinisch relevante problemen aan te kaarten en op een correcte manier in het daglicht te plaatsen. Ook werd het me gaandeweg duidelijker dat de oplossingen voor de PSA problematiek aan draagkracht winnen wanneer ze gestut worden door wetenschappelijke argumenten. Voor die leerschool ben ik een aantal mensen schatplichtig.

In de eerste plaats zijn dat mijn beide promotoren.

Professor Zimmermann, beste Luc! Hartelijk dank omdat ik de ruimte kreeg om los van de andere onderzoekslijnen in onze vakgroep, toch mijn weg te mogen gaan. Ook al behoorde de inhoud van mijn werk niet tot jouw expertise, je commentaren op mijn artikelen kwamen snel en waren steeds zeer bruikbaar.

Professor Knape, beste Hans! Je enorme ervaring, je jarenlange expertise op het vlak van PSA en je manier van 'networking' waren van groot belang voor zowel mijn werk met de richtlijn als met dit promotietraject. Bovenal was je support zeer metaforisch voor de ideale PSA wereld: een anesthesioloog die een niet-anesthesioloog inspireert om het vooral zelf te doen... Mijn dank is groot! Ik hoop van harte dat we samen nog een heel eind mogen komen met het implementeren van de richtlijn.

Doctor Nieman, beste Fred! Op een dag kwam ik je werkkamer binnen met mijn versie van een enquête die ik de week nadien naar de Nederlandse kinderartsen wou sturen. Je zou er naar kijken en dan zouden we even door je commentaren gaan. Dat laatste liep een beetje uit de hand en pas een klein jaar later (...) was je tevreden met het resultaat. Men had me gewaarschuwd dat je een moeilijk en veeleisend methodoloog was. Van dat eerste heb ik niets gemerkt, terwijl dat tweede me geen windeieren heeft gelegd. Ik overdrijf helemaal niet door te stellen dat zonder jou dit proefschrift er nooit was geweest. Bovendien heb je mij met veel geduld een blik gegund op een tot dan voor mij volledig onbekende wereld, namelijk die van de sociale wetenschappen. En tijdens die tientallen uren tussen de stapels documenten en de SPSScalculaties weidden we gretig uit over zoveel meer. Over wetenschapsfilosofen, maar ook over de Tour de France; over de Belgische politiek, maar ook over andere 'comics'; over de dwaze verrechtsing in dit Nederland, maar ook over Kuifje in het Brussels en - niet te vergeten! - Piet Fluwijn en Bolleke; en natuurlijk ook over palindromen! In girum imus nocte et consumimur igni!

De leden van de leescommissie (de hoogleraren van Kleef, de Groot en Neef en dr. Willigers) kregen met dit overmaatse proefschrift in het begin van de zomer van 2011 een taaie klus 
voorgeschoteld. Toch kwam hun oordeel snel. Mijn excuses voor zoveel leeslast, maar bovenal mijn grote dank om in 'mijn' leescommissie te willen zetelen. Twee leden van de leescommissie ben ik bijzondere dank verschuldigd. Het was professor de Groot die mij in november 2005 bij de presentatie op het jaarlijkse NVK congres van de eerste resultaten van onze Maastrichtse sedatie unit aanmoedigde om onze oplossing landelijk uit te dragen. Volgens jou was PSA in Nederland slecht geregeld. Je bleek overmaat van gelijk te hebben. We staan intussen ruim 6 jaar verder en ik ben zeer verheugd, beste Ronald, je bij deze eerste mijlpaal weer te kunnen begroeten. Kinderanesthesioloog Henriette Willigers was dan weer van bij het begin als consulent betrokken bij het PSA project van het kinder-IC team in Maastricht. Vooral het vertrouwen dat we via haar van de vakgroep anesthesie kregen, is van levenbelang gebleken voor dit project.

Ook de overige leden van de corona (de hoogleraren Benoit, van Vught en Vles en dr.Vos) dank ik graag voor hun enthousiaste toezegging om deze belangrijke rol op zich te willen nemen.

Voor het verrichten van het onderzoek en bij het schrijven van de artikelen kon ik rekenen op de specifieke deskundigheid van een aantal mensen. Sera Schniedermeier was in 2006 en 2007 van onschatbaar groot belang om de tienduizenden data die de enquête had opgeleverd te verwerken in degelijke SPSS files. Zelf had ik daar nooit de tijd en het geduld voor gehad. Jonathan Verbeke, kinderradioloog aan het VU Medisch Centrum Amsterdam, had een belangrijke rol bij het schrijven van het artikel over PSA voor MRI bij kinderen. Studente geneeskunde Elke van Beek ging voortvarend de strijd aan met de vakliteratuur om daaruit de 'best evidence' te filteren voor onze systematic review over PSA bij endoscopische onderzoeken. Rankie ten Hoopen, docent gezondheidsrecht bij de faculteit geneeskunde van de universiteit Maastricht, was absoluut onmisbaar om het gebruik van dwang en fixatie tijdens procedures bij kinderen in de juiste juridische context te plaatsen. Dat ze bovendien een uitgesproken voorliefde voor taalkundige zuiverheid heeft, heb ik bijzonder geapprecieerd.

Maar er zijn nog zoveel meer mensen die ik danken moet. Met het reële risico dat ik mensen vergeet, som ik ze hier op:

Monique Engel, Arno Brouwers, Dick van Waardenburg en Gijs Vos, mijn directe collega's op de PICU. Het schrijven van dit proefschrift heeft me vaker dan goed was afgeleid van het echte werk. Dank voor jullie geduld én aanmoedigingen. Mede dankzij jullie is PSA intussen een van de kwaliteitsproducten van onze afdeling geworden. Dat jullie bovendien stuk voor stuk briljante en kundige dokters zijn, hebben Ann, ik en Emilie vorig jaar aan den lijve moeten/mogen ondervinden. Onze eeuwige dank daarvoor!

Dat laatste geldt ook voor de PICU verpleging. Ada, Anja, Annet, Bianca, Bianca, Charlotte, Corine, Diana, Dominique, Elly, Eugenie, Fritzi, Ilse, Karoline, Kim, Liesbeth, Marie-Jose, Michelle, Mieke, Peggy, Resi, Sandra, Alex, François, Hay, Henk, Jos, Michiel, Ron, Ronald, Ronald en Ruud: dat jullie kwaliteiten moeilijk te overschatten zijn weet ik nu ook uit persoonlijke ervaring. Niet alleen vormen jullie de onmisbare kern van de hoge zorgkwaliteit die we als PICU leveren, jullie zijn bovendien van in het begin super enthousiast gaan meerijden op de PSA trein. Heel veel dank daarvoor.

Het team secretaresses van het secretariaat kindergeneeskunde dank ik voor alle hulp bij de praktische en administratieve romplomp die het maken van een proefschrift met zich meebrengt. Heel in het bijzonder gaat mijn dank uit naar Anja Lemmerlijn.

Nicolle Cobben en Margriet Martens, de collega's van het Centrum voor Thuisbeademing, en Jan schieveld, kinderpsychiater en coördinator van het onderzoek naar delier bij kinderen, dank ik voor hun aanmoedigingen.

Vivian van Thoor-Wijnands en Lavienja Schreurs-Braam, mijn paranimfen. Dankjewel voor jullie hulp bij de voorbereiding, jullie 'acte de présence', maar bovenal voor jullie vriendschap. 
En naarmate het danklijstje opschuift, des te wezenlijker worden de genoemde mensen. Mijn ouders, als eerste. Ik ben jullie een leven lang dankbaar voor wie ik dankzij jullie kon worden. Weet je nog hoe ik vroeger was: een schoolziek kind dat van orde noch van studeren hield. Uiteindelijk is slechts alleen dat eerste hopeloos gebleven... Dank voor jullie liefde, levenslessen en wijsheid.

Broers en zussen, alle vrienden en andere mensen die ik tot nu toe heb mogen ontmoeten. Ik dank hen voor hun vriendschap, inspiratie en liefde en voor hun belangrijke bijdragen aan mijn leven.

Pauline, Lukas en Emilie. Jullie zijn onze enige echte rijkdom. Het is een ongelooflijk voorrecht om jullie te mogen zien bloeien en groeien.

En tenslotte, Ann, mijn lieve Ann. Deze strijd is nu voorbij. Kom maar in mijn armen. Wie je bent en wat we hebben is onbeschrijfelijk (en) mooi. Hoe dichter bij de essentie van het leven, hoe meer de woorden falen. Dus laat ik nu maar heel intens gaan zwijgen... 


\section{Curriculum Vitae}

Piet Leroy werd geboren op 1 januari 1969 in Deinze (België). In 1986 voltooide hij de middelbare school (Latijn-Griekse humaniora) aan het St.-Hendrikscollege te Deinze. Tussen 1986 en 1993 volgde hij de opleiding Geneeskunde aan de Universiteit Gent (UG). Hij behaalde in 1989 het diploma Bachelor in de geneeskundige wetenschappen met de 'Grootste Onderscheiding' en in 1993 het diploma Doctor in de Genees- Heel- en Verloskunde eveneens met de 'Grootste Onderscheiding'. Hij ontving in 1993 de eerste prijs van de Faculteit Geneeskunde (UG) voor de bijzondere wijze van onderscheiden tijdens de gehele opleiding.

Tussen 1993 en 1998 volgde hij de specialisatieopleiding tot kinderarts (opleider Prof. Dr. JG Leroy) aan de Universitaire Kliniek voor Kinderziekten (UG). Tijdens deze opleiding volgde hij ook stages aan de afdelingen kindergeneeskunde van het Algemeen Ziekenhuis St.-Jan te Brugge (opleider Dr. JP Van Biervliet) en het Wilhemina Kinderziekenhuis te Utrecht (opleiders Prof. Dr. AJ van Vught en Prof. Dr. A Okken).

Tussen 1998 en 2000 volgde hij een bijkomende specialisatie in de pediatrische intensieve zorgen, achtereenvolgens in het Sophia Kinderziekenhuis (Rotterdam; opleider Prof. Dr. D Tibboel) en in het Radboud Universitair Medisch Centrum (Nijmegen; opleider dr. LGFM van 't Hek). Sinds april 2001 werkt hij als kinderintensivist en staflid op de afdeling kindergeneeskunde van het Maastricht Universitair Medisch Centrum (MUMC). Zijn bijzondere aandachtsgebieden zijn procedurele sedatie en analgesie, delier bij ernstig zieke kinderen, thuisbeademing en reanimatie.

Daarnaast vervult hij verschillende onderwijskundige taken. Binnen de Faculty of Health, Medicine and Life Sciences (FHML) van de Universiteit Maastricht heeft hij als tutor, coach en observator gefunctioneerd. Tevens is hij trainer binnen de opleiding 'Basic Clinical Teaching', lid van de planningsgroep van het 'Tropenblok', coördinator van het blok 'Regulatie en Integratie' in het eerste jaar geneeskunde en lid van de onderwijscommissie van de vakgroep kindergeneeskunde. In 2005 werd hij door de studenten verkozen tot 'beste coach' van het cluster 'circulatie en Longen' en in 2010 werd hij door de co-asssitenten van de UM verkozen tot 'Clinicus van het jaar'.

Buiten de faculteit is hij trainer in de 'Advanced Pediatric Life Support' (Stichting Spoedeisende Hulp bij Kinderen (SSHK); Tilburg) en in de 'Advanced Trauma Life Support' (Stichting ATLS, Tilburg). Sinds 2011 is hij voorzitter van het expert committee van de SSHK.

Tussen 2006 en 2010 was hij namens de Nederlandse Vereniging voor Kindergeneeskunde voorzitter van de werkgroep "Procedurele sedatie en/of analgesie bij Kinderen buiten de operatiekamer". Deze multidisciplinaire werkgroep, die functioneerde binnen het CBO Kwaliteitsin- 
stituut voor de gezondheidszorg, publiceerde een evidence based richtlijn die aanbevelingen formuleert ten aanzien van de Procedurele Sedatie en/of Analgesie (PSA) bij kinderen. In de marge van dit richtlijnwerk werd in het najaar van 2006 gestart met het onderzoek dat tot dit proefschrift heeft geleid.

Piet Leroy is sinds 1999 gehuwd met Ann Vanderhaeghe en samen hebben zij drie schatten van kinderen: Pauline ( $\left.{ }^{\circ} 2003\right)$, Lukas ( $\left.{ }^{\circ} 2005\right)$ en Émilie ( $\left.{ }^{\circ} 2008\right)$. 


\section{List of Publications}

\section{International Papers}

Additional use of methotrimeprazine for treating refractory agitation in pediatric patients. van der Zwaan S, Blankespoor RJ, Wolters AM, Creten C, Leroy PL, Schieveld JN. Intensive Care Med. 2011 Nov 23. [Epub ahead of print].

Safe and effective procedural sedation for gastro-intestinal endoscopy in children: a systematic review. Van Beek EJ*, Leroy PL*. J Pediatr Gastroenterol Nutr 2011 Oct 3 [Epub ahead of print]. (*first authors)

Pediatric delirium in the pediatric intensive care unit: a systematic review and an update on key issues and research questions. Creten C, Van Der Zwaan S, Blankespoor R, Leroy PL, Schieveld JN. Minerva Anestesiol. 2011 May 26. Nov;77(11):1099-107. Epub 2011 May 26.

On the utility of diagnostic instruments for pediatric delirium in critical illness: an evaluation of the Pediatric Anesthesia Emergence Delirium Scale, the Delirium Rating Scale 88, and the Delirium Rating Scale-Revised R-98.Janssen NJ, Tan EY, Staal M, Janssen EP, Leroy PL, Lousberg R, van Os J, Schieveld JN. Intensive Care Med. 2011 May 13. [Epub ahead of print]

Procedural sedation for paediatric neuroimaging: time to move on! Leroy PL, Knape JT. Arch Dis Child. 2011; Letter to the Editor - Published on line $-10^{\text {th }}$ of February 2011.

Medical errors: the importance of the bullet's blunt end. Leroy P. Eur J Pediatr. 2011 Feb;170(2):251-2. Epub 2010 Sep 1.

Professional skills and competence for safe and effective procedural sedation in children: recommendations based on a systematic review of the literature. Leroy PL, Schipper DM, Knape HJ. Int J Pediatr. 2010;201

Fatal cerebral edema associated with serine deficiency in CSF. Keularts IM, Leroy PL, RubioGozalbo EM, Spaapen L, Weber B, Dorland B, de Koning TJ, Verhoeven-Duif NM. J Inherit Metab Dis. 2010 Mar 19. [Epub ahead of print]

Late respiratory failure in Jeune syndrome. Leroy P, Martens M, Schott N, Cobben N. Eur J Pediatr. 2010 Mar;169(3):375-6. Epub 2009 Aug 27. No abstract available. 
Adherence to safety guidelines on paediatric procedural sedation: the results of a nationwide survey under general paediatricians in The Netherlands. Leroy PL, Nieman FH, Blokland-Loggers HE, Schipper DM, Zimmermann L, Knape JT. Arch Dis Child. 2010 Dec;95(12):1027-30. Epub 2009 Sep 29.

Prolonged stay at the paediatric intensive care unit associated with paediatric delirium. Smeets IA, Tan EY, Vossen HG, Leroy PL, Lousberg RH, van Os J, Schieveld JN. Eur Child Adolesc Psychiatry. 2010 Apr;19(4):389-93. Epub 2009 Sep 27.

Diagnostic considerations regarding pediatric delirium: a review and a proposal for an algorithm for pediatric intensive care units. Schieveld JN, van der Valk JA, Smeets I, Berghmans E, Wassenberg R, Leroy PL, Vos GD, van Os J. Intensive Care Med. 2009 Nov;35(11):1843-9. Review.

Severe hemolysis and methemoglobinemia following fava beans ingestion in glucose-6phosphatase dehydrogenase deficiency: case report and literature review. Schuurman $M$, van Waardenburg D, Da Costa J, Niemarkt H, Leroy P. Eur J Pediatr. 2009 Jul;168(7):779-82. Epub 2009 Mar 5. Review.

Procedural sedation and analgesia in children by non-anesthesiologists in an emergency department. Leroy PL, Gorzeman MP, Sury MR. Minerva Pediatr. 2009 Apr;61(2):193-215. Review.

Minimizing limb amputations in meningococcal sepsis by early microsurgical arteriolysis. Boeckx WD, Nanhekhan L, Vos GD, Leroy P, Van den Kerckhove E. J Pediatr Surg. 2009 Aug;44(8):162530.

Vanishing spleen after Nissen fundoplication: a case report. Dijkman KP, van Heurn LW, Leroy PL, Vos GD. Eur J Pediatr. 2009 Mar;168(3):355-7. Epub 2008 Jul 3.

Pediatric illness severity measures predict delirium in a pediatric intensive care unit. Schieveld JN, Lousberg R, Berghmans E, Smeets I, Leroy PL, Vos GD, Nicolai J, Leentjens AF, van Os J. Crit Care Med. 2008 Jun;36(6):1933-6.

Acute hepatic injury in four children with Dravet syndrome: valproic acid, topiramate or acetaminophen? Nicolai J, Gunning B, Leroy PL, Ceulemans B, Vles JS. Seizure. 2008 Jan;17(1):92-7. Epub 2007 Aug 13.

Pets or meds: how to tackle misery in a paediatric intensive care unit. Vos GD, van Os J, Leroy PL, Schieveld JN. Intensive Care Med. 2007 Aug;33(8):1492-3. Epub 2007 Jun 19. No abstract available.

Pediatric delirium in critical illness: phenomenology, clinical correlates and treatment response in 40 cases in the pediatric intensive care unit. Schieveld JN*, Leroy PL*, van Os J, Nicolai J, Vos GD, Leentjens AF. Intensive Care Med. 2007 Jun;33(6):1033-40. Epub 2007 Apr 25. (*first authors)

Long-term risk of atypical fungal infection after near-drowning episodes. Leroy $\mathbf{P}$, Smismans $\mathbf{A}$. Pediatrics. 2007 Feb;119(2):417; author reply 417-8.

Invasive pulmonary and central nervous system aspergillosis after near-drowning of a child: case report and review of the literature. Leroy P, Smismans A, Seute T. Pediatrics. 2006 Aug;118(2):e509-13. Epub 2006 Jul 24. Review. 
Staphylococcus aureus relapsing bacteremia originating from endovascular thrombi. Leroy $\mathbf{P}$, Gavilanes A, Vink C, Jacobs J. Pediatr Infect Dis J. 2005 Feb;24(2):185-7.

High-frequency oscillatory ventilation in RSV bronchiolitis patients. Duval EL, Leroy PL, Gemke RJ, van Vught AJ. Respir Med. 1999 Jun;93(6):435-40.

\section{National Papers}

Succesvolle en veilige sedatie voor MRI-onderzoek bij kinderen. De dagelijkse praktijk in Nederland getoetst aan de resultaten van een systematic review van de literatuur. Leroy PL, Verbeke JIML, Nieman FHM, Blokland-Loggers HEA. Tijdschrift voor Kindergeneeskunde. 2011 Apr;79(3):42-53

Procedurele sedatie en/of analgesie bij Kinderen: van de schemerzone naar het daglicht. Leroy P. Praktische Pediatrie 2010 Sept;3:188-191

De behandeling van ernstige diabetische keto-acidose bij kinderen: magie of wetenschap? Leroy PL, De Vroede M. Praktische Pediatrie. 2009 Sept; 3: 88-194

Screening en follow-up van kinderen die voor thuisbeademing in aanmerking komen. Leroy PL, Knoester H, Cobben N. Tijdschrift voor Kindergeneeskunde. 2009 Jun;77(3):42-53

[Delirium in critically ill children in a paediatric intensive care unit]. Schieveld JN, Leroy PL, Leentjens AF. Ned Tijdschr Geneeskd. 2006 Jul 15;150(28):1545-8.

[An unsuspected imported disease: meningo-encephalitis contracted in Spain]. Thijssen HS, Leroy PL, van 't Hek LG, Hurkx GA. Ned Tijdschr Geneeskd. 2004 Jan 17;148(3):113-7. Dutch

\section{Guidelines}

Piet Leroy en Monique de Vroede. Behandeling van Diabetische Keto-acidose en Hyperosmolair Hyperglycemisch Syndroom (NVK richtlijn 2012)

Piet Leroy et al. Procedurele Sedatie en/of Analgesie (PSA) bij kinderen buiten de operatiekamer. Evidence based richtlijn 2009. CBO Kwaliteitsinstituut voor de Gezondheidszorg.

\section{Books}

Advanced Pediatric Life Support. De Nederlandse Editie. Derde druk. Redactie Nigel M Turner en Piet L Leroy . december 2011, (c) Reed Business

Mike RJ Sury, Piet UM Leroy. Paediatric Sedation: the European experience and approach. Chapter 17 in Keira P. Mason (ed.), Pediatric Sedation Outside of the Operating Room: A Multispecialty International Collaboration, DOI 10.1007/978-0-387-09714-5_17, (C Springer Science+ Business Media, LLC, 2011. 